\title{
Structure in the Near Field of the Transverse Jet
}

Thesis by

Thomas Frank Fric

In Partial Fulfillment of the Requirements for the Degree of Doctor of Philosophy

California Institute of Technology

Pasadena, Califomia

1990

(Submitted April 13, 1990) 
(C) 1990

Thomas Frank Fric

All Rights Reserved 
iii

dedicated to my parents 


\section{Acknowledgements}

I am grateful to my advisor, Professor Anatol Roshko, for making this research possible. His constant enthusiasm and support for my work and ideas are appreciated. Professionally, I am thankful for his insights into fluid mechanics. I also thank him for being a friend.

In addition, I acknowledge the many members of GALCIT (faculty, staff, and students) who have contributed to various aspects of this research, making life easier for me. In particular, I would like to thank Professor Hans Hornung for many helpful discussions on the issue of vorticity generation.

I would like to thank the friends I have made while at Caltech for many great memories which will last. Special thanks go out to the Supper Club (Ruth, Mass, Rick, Shelly, Jim, Shenda, and Karen) and Bob Walker.

Finally, I thank my family, whose support is always appreciated.

This research was funded by the Office of Naval Research, under contract \#N00014-85-K-0646 and grant \#N00014-89-J-1991. 


\begin{abstract}
Photographs of an axisymmetric turbulent jet issuing from a wall into a crossflow display the four types of vortical structures which exist in the near field: the jet shear layer vortices, the nascent far field vortex pair, the near wall horseshoe vortices, and a system of vortices in the wake of the jet.

Additionally, results of hot-wire measurements in the wake of the transverse jet are presented. Among these results are characteristic wake Strouhal frequencies, which vary with the jet to crossflow velocity ratio, and wake velocity profiles.

It is found that the wake vorticity is not "shed" from the jet but is formed from vorticity which originated in the wall boundary layer. Therefore, analogies between the wakes of transverse jets and the wakes of solid cylinders are incorrect. Since the jet is not a solid obstacle to the crossflow, as a cylinder is, new vorticity is not generated at the interface between the jet and the crossflow. Instead, the boundary layer on the wall from which the jet issues separates near the downstream side of the jet because it cannot negotiate the adverse pressure gradient imposed on it by the flow around the jet, which is not "separated" as it is for a cylinder. The wake vortices subsequently formed are found to be most coherent near a jet to crossflow velocity ratio of four.

The near field development of the counterrotating vortex pair, which is the dominant structure of the far field jet, is also addressed. It is argued that the source of vorticity for the vortex pair is the vorticity from the boundary layer within the jet nozzle. Estimates for the strength of these vortices are obtained by considering the flux of vorticity emanating from the nozzle.
\end{abstract}

Possible implications for mixing are briefly discussed. 


\section{Contents}

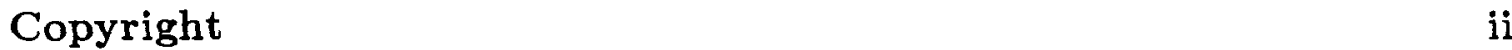

Dedication

Acknowledgements iv

Abstract $\quad$ v

List of Tables $\quad$ x

List of Figures $\quad$ xi

List of Symbols $\quad$ xviii

1 Introduction $\quad 1$

1.1 Fluid mechanical motivation . . . . . . . . . . . . 2

1.2 A review of transverse jet research . . . . . . . . . . 3

1.3 What is the near field? . . . . . . . . . . . . 6

1.4 A preview ....................... 7

2 Experimental set-up $\quad 9$

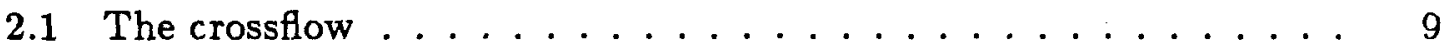

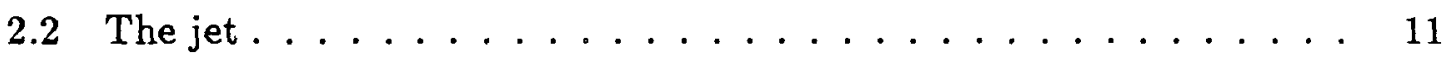

2.3 Flow visualization ...................... 13 
2.4 Measurement apparatus .................. 15

2.5 Nominal vs. actual velocities ................ 16

3 Near field flow visualization $\quad 17$

3.1 Structure of the deflected jet ................. 18

3.1.1 Distorted jet shear layer vortices ............ 18

3.1.2 Counterrotating vortex pair ............. 21

3.2 Structure at the crossflow wall ............... 22

3.2.1 Horseshoe vortices and more . . . . . . . . . . . . 22

3.3 Wake structure .................... 24

4 Sources of vorticity 29

4.1 The vorticity transport equation .............. 30

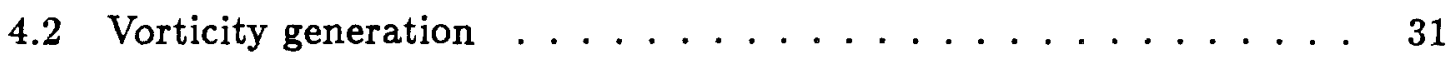

4.3 Implications . . . . . . . . . . . . . . . . 34

5 Wake of the transverse jet $\quad 35$

5.1 Source of wake vorticity and wake formation model . . . . . . . 36

5.1.1 Smoke as a vorticity marker . . . . . . . . . . 37

5.1.2 Observations of crossflow boundary layer "separation events" 39 5.1.2.1 Wake flow patterns ........... 42

5.1.3 Wake vortices and separation events - Comparing characteristic (Strouhal) frequencies ............. 44

5.1.4 Wake velocity profile effects of thickening the crossflow boundary layer. . . . . . . . . . . . . . . . 45

5.1.5 Why the crossflow boundary separates/Wake formation model 48

5.1.6 The principal message .............. 51 
5.2 Additional wake measurements . . . . . . . . . . . 51

5.2.1 Characteristic wake (Strouhal) frequencies ........ 51

5.2.1.1 Some conventions for $S t_{w} \ldots \ldots \ldots 52$

5.2.1.2 Parameter dependency ........... 53

5.2.1.3 Comparison to others ............ 56

5.2.1.4 Cylinder $S t_{w}$ comparison ........... 57

5.2.2 More from the wake $\left(U / U_{c f}\right)$ profiles . . . . . . . . . 57

5.2.3 Wake total pressure measurements along $Y / D_{j}=0 \ldots 58$

6 Near field development of the counterrotating vortex pair (CVP) 60

6.1 CVP - A near field structure .............. 61

6.2 The approach - Conservation of jet vorticity flux ........ 62

6.3 Vorticity flux analysis ................. 63

6.3.1 $\Phi_{1}$ by hypothesis ........................ 64

6.3.2 An estimate for $U_{1 c o n v} \ldots \ldots \ldots 7$

6.4 Present result and comparison with others . . . . . . . . . 69

7 Discussions and summary $\quad 72$

7.1 The misunderstood wake ................ 72

7.1.1 Kinematics vs. dynamics ............. 73

7.1.2 Rate of strain vs. vorticity ............. 74

7.1.3 Total pressure gradients .............. 75

7.1.4 Guideline for vorticity generation . . . . . . . . . 75

7.2 Dependence of the wake on $V R \ldots \ldots$

7.2.1 More on $V R$ effects ............... 77

7.2.2 Why are $V R \approx 4$ wakes special? . . . . . . . . 79

7.3 What sets $f_{w} \ldots \ldots \ldots \ldots \ldots$ 
7.3.1 Cylinder wake modes ............... 82

7.3.2 Possible transverse jet modes . . . . . . . . . . . . 83

7.4 Entrainment and mixing ............... 85

7.4.1 Transverse jets make better mixers . . . . . . . . . 86

7.4.2 The role of the wake ............. 87

7.4.3 The role of the counterrotating vortex pair ....... 88

7.5 Variations on the theme ............... 89

7.5.1 Protruding jet ................... 89

7.5.2 Crossflow boundary layer alterations .......... 90

7.5 .3 Jet alterations ..................... 90

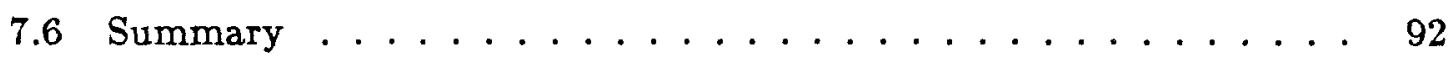

A Applications of transverse jets $\quad 94$

$\begin{array}{ll}\text { B Measurement details } & 97\end{array}$

B.1 Crossflow boundary layer thicknesses . . . . . . . . . . . 97

B.2 Jet boundary layer thicknesses at the nozzle exit . . . . . . . . 98

B.3 Power Spectra Details . . . . . . . . . . . . . . 98

B.4 $U_{1 \text { conv }}$ estimates from total pressure measurements . . . . . . . 99

$\begin{array}{ll}\text { References } & 101\end{array}$

$\begin{array}{ll}\text { Tables } & 108\end{array}$

$\begin{array}{ll}\text { Figures } & 116\end{array}$ 


\section{List of Tables}

TABLE 2.1: Control screen(s) used at the end of the wind tunnel test section.

TABLE 2.2: Characteristics of the crossflow boundary layer at $X / D_{j}=0$. Nominal values are shown, i.e., no jet is present.

TABLE 2.3: Jet characteristics at $Z / D_{j}=0$. All values shown are nominal, i.e., there is no crossflow. Jet is issuing into a closed wind tunnel.

TABLE 2.4: Comparison of nominal and actual jet to crossflow velocity ratios. ( $U_{c f}$ for actual values was measured at $X / D_{j}=-15$, not 0 .)

TABLE 5.1a: Measured estimates for wake displacement and momentum thicknesses. $V R=4, \operatorname{Re}_{c f}=3800$. $*$ indicates wake-like profile.

TABLE 5.1b: Measured estimates for wake displacement and momentum thicknesses. $V R=8, R e_{c f}=3800$. * indicates wake-like profile.

TABLE 5.1c: Measured estimates for wake displacement and momentum thicknesses. Cylinder of $A R=6, R e_{c f}=3800$. * indicates wake-like profile.

TABLE 5.2: Averages for wake displacement and momentum thicknesses using wake-like profiles from tables 5.1 only. $R e_{c f}=3800$.

TABLE B.1: HP 3582A Spectrum Analyzer sampling characteristics.

TABLE B.2: Camera location for photographs in which jet "issues at the viewer." 


\section{List of Figures}

FIGURE 1.1: Counterrotating vortex pair structure of the transverse jet in the far field.

FIGURE 1.2: Structure in the near field of the transverse jet.

FIGURE 2.1: 20" X 20" open return wind tunnel. From Cimbala (1984). (Not drawn to scale).

FIGURE 2.2: Wind tunnel contraction section modification.

FIGURE 2.3: Detail of the test section with jet injected at $90^{\circ}$.

FIGURE 2.4: Schematic of the jet delivery system (not drawn to scale).

FIGURE 2.5: Smoke-wire traversing set-up (not drawn to scale).

FIGURE 2.6: Typical smoke-wire/jet orientations.

FIGURE 3.1: Leading edge of the distorted shear layer structure of the deflected jet.

(a) $V R=2$, (b) $V R=4$, (c) $V R=6$, (d) $V R=8$, (e) $V R=10$.

$R e_{c f}=3800 . Y_{s w} / D_{j}=0$.

FIGURE 3.2: Close-up of the leading edge of the deflected jet showing axial flow along the tilted and distorted vortex rings. $V R=2 . R e_{c f}=3800$.

$Y_{s w} / D_{j}=0$.

FIGURE 3.3: Streakline pattern with the jet issuing at the viewer shows entrainment of crossflow fluid. (a) $V R=2 . R e_{c f}=7600 . Z_{s w} / D_{j}=.75$,

(b) $V R=10 . R e_{c f}=3800 . Z_{s w} / D_{j}=.5$.

FIGURE 3.4: Side view of the deflected jet. (a) $V R=2$, (b) $V R=4$, (c) $V R=6$, (d) $V R=8$, (e) $V R=10$. $R e_{c f}=3800$.

FIGURE 3.5: Section at $X / D_{j}=1$ shows early development of the counterrotating vortex pair, as viewed from $X / D_{j}>1$. The drawing at right indicates the section visualized. $V R=4 . R e_{c f}=3800 . Z_{s w} / D_{j}=0+$. 
FIGURE 3.6: Streaklines showing entrainment of crossflow fluid by the vortex pair. The drawing clarifies the flow pattern. $V R=6 . R e_{c f}=3800$. $Y_{s w} / D_{j}=1$.

FIGURE 3.7: Development of the jet cross section with distance from the crossflow wall. Jet issues at the viewer. (a) $V R=2$. $R e_{c f}=3800$, (b) $V R=4$. $R e_{c f}=3800$, (c) $V R=6 . R e_{c f}=11400$, (d) $V R=8$. $R e_{c f}=3800,(\mathrm{e}) V R=10 . R e_{c f}=7600$.

FIGURE 3.8: Horseshoe vortex system at the crossflow wall. Jet issues at the viewer. (a) $V R=2$, (b) $V R=4$, (c) $V R=6$, (d) $V R=8$, (e) $V R=10$. $R e_{c f}=3800 . Z_{\mathrm{sw}} / D_{j}=0+$.

FIGURE 3.9: Horseshoe vortex system for a wall-mounted circular cylinder of $A R=6 . R e_{c f}=3800 . Z_{s w} / D_{j}=0+$.

FIGURE 3.10: $Y I D_{j} \approx 0$ cross section of the horseshoe vortices just ahead of the jet. (a) $V R=2$, (b) $V R=10 . R e_{c f}=3800 . Y_{s w} D_{j}=0$.

FIGURE 3.11: Side view of the wake vortices, which extend from the crossflow wall to the jet. (a) $V R=2$, (b) $V R=4$, (c) $V R=6$, (d) $V R=8$, (e) $V R=10 . R e_{c f}=3800 . Z_{s w} / D_{j}=0+$.

FIGURE 3.12: Entrainment of crossflow fluid by the wake structures.
(a) $V R=2 . Y_{s w} / D_{j}=.5$, (b) $V R=4 . Y_{s w} / D_{j}=1.5$,
(c) $V R=6 . Y_{s w} / D_{j}=1$, (d) $V R=8 . Y_{s w} / D_{j}=1$,
(e) $V R=10 . Y_{s w} J D_{j}=1$. $R e_{c f}=3800$.

FIGURE 3.13: Side view of wake vortices. (a) $V R=8 . Y_{s w} D_{j}=-.5$, (b) $V R=10$. $Y_{s w} / D_{j}=.5 . R e_{c f}=3800 . X_{s w} / D_{j}=2$.

FIGURE 3.14: Reverse flow region at the near wall, very near wake for (a) $V R=2$, (d) $V R=8$ and (e) $V R=10$. No reverse flow is observed for (b) $V R=4$ and (c) $V R=6 . \quad R e_{c f}=3800$. $X_{s w} / D_{j}=2 . Y_{s w} / D_{j}=0$.

FIGURE 3.15: Cross sectional views of the wake at various distances from the crossflow wall. Jet issues at the viewer. (a) $V R=2$, (b) $V R=4$, (c) $V R=6$, (d) $V R=8$, (e) $V R=10$. $R e_{c f}=3800$.

FIGURE 3.16: Simultaneous cross sectional and side view of the wake vortices. $V R=4 . R e_{c f}=3800 . Z_{s w} / D_{j}=0+$. 
FIGURE 3.17: Effects of crossflow Reynolds number on near wall wake flow.

(a) $R e_{c f}=3800$, (b) $R e_{c f}=7600$, (c) $R e_{c f}=11400$.

$V R=6 . Z_{s w} / D_{j}=0+$.

FIGURE 5.1: Comparison of a circular cylinder wake with the wake of a transverse jet. (a) $A R=6$ cylinder, (b) $V R=4$ transverse jet. $R e_{c f}=7600$. $Z_{s w} / D_{c}=Z_{s w} / D_{j}=1$.

FIGURE 5.2: Using smoke as a vorticity marker. (a) Jet is tagged with smoke, (b) Smoke is initially in the crossflow boundary layer. The view in (b) is identical to that in (a). $V R=4 . R e_{c f}=3800$.

FIGURE 5.3: Separation event and the accompanying near wall flow. Smokewire flow visualization $\left(Z_{s w} / D_{j}=0+\right)$. Arrow indicates a separation event. $V R=4$. $R e_{c f}=3800$.

FIGURE 5.4: Sketch highlighting the structures seen in the photograph of figure 5.3.

FIGURE 5.5: Simultaneous cross sectional view (bottom) and side view (top) of the wake vortices. The arrows indicate the same separation event in each view. $V R=4 . R e_{c f}=3800 . Z_{s w} / D_{j}=0+$.

FIGURE 5.6: Typical near wall, very near wakes showing crossflow boundary layer separations. (a) $V R=2$, (b) $V R=4$, (c) $V R=6$, (d) $V R=8$, (e) $V R=10 . R e_{c f}=3800 . Z_{s w} / D_{j}=0+$.

FIGURE 5.7: Examples where separation events are most clearly evident.

(a) $V R=4$, (b) $V R=6$, (c) $V R=8$, (d) $V R=10$.

$R e_{c f}=3800 . Z_{s w} / D_{j}=0+$.

FIGURE 5.8: Instantaneous crossflow wall surface topology/streamline pattern for the flow shown in figure 5.3. Separation lines: $A B, A^{\prime} B^{\prime}, A^{\prime \prime} B^{\prime \prime}$. Attachment lines: $\mathrm{AC}, \mathrm{A}^{\prime} \mathrm{C}^{\prime}, \mathrm{A}^{\prime \prime} \mathrm{C}^{\prime \prime}$.

FIGURE 5.9: Flow streamsurfaces associated with a separation event.

FIGURE 5.10: Two wake vortices attached at the trailing edge of the jet.

$V R=$ 5.8. $R e_{c f}=3800 . Z_{s w} / D_{j}=0+$. 
FIGURE 5.11: Comparison of $S t_{w}$ with $S t_{s e p}$. (a) $R e_{c f}=3800$, (b) $R e_{c f}$ $=7600$, (c) $R e_{c f}=11400 . S t_{w}$ measured at $X / D_{j}=3.5, Y / D_{j}$ $=1.5, Z I D_{j}=.5$. $S t_{\text {sep }}$ measured near the crossflow boundary layer separation events.

FIGURE 5.12: Wake velocity profiles at $Z I D_{j}=.5 . V R=4 . R e_{c f}=3800$.

FIGURE 5.13: Wake velocity profiles at $Z / D_{j}=2.5 . V R=4 . R e_{c f}=3800$.

FIGURE 5.14: Wake velocity profiles at $Z I D_{j}=4.5 . V R=4 . R e_{c f}=3800$.

FIGURE 5.15: Wake velocity profiles at $Z / D_{j}=.5 . V R=8 . R e_{c f}=3800$.

FIGURE 5.16: Wake velocity profiles at $Z I D_{j}=2.5 . V R=8 . R e_{c f}=3800$.

FIGURE 5.17: Wake velocity profiles at $Z / D_{j}=4.5 . V R=8 . R e_{c f}=3800$.

FIGURE 5.18: Side view of flow about a circular cylinder. $A R=6 . R e_{c f}=3800$. $Z_{s w} / D_{j}=0+$.

FIGURE 5.19: Cross sectional views of wakes with $Z_{s w}{ }^{\prime} D_{j}=Z_{s w}{ }^{\prime} D_{c}=0+$. $R e_{c f}=11400$.

FIGURE 5.20: Cross sectional views of wakes with $Z_{s w} / D_{j}=Z_{s w} / D_{c}=.5$. $R e_{c f}=11400$.

FIGURE 5.21: Comparison of flow around a cylinder with the flow around a jet. $R e_{c f}=3800$.

FIGURE 5.22: Predicted separations using a Thwaites method calculation of the crossflow boundary layer with a potential flow pressure gradient imposed on it.

FIGURE 5.23: Definitions of assigned spectral sharpness levels. (a) sharpness $=0$, (b) sharpness $=1$, (c) sharpness $=2$, (d) sharpness $=3$,

(e) sharpness $=4$.

FIGURE 5.24: The degree of repeatibility of the wake Strouhal number data. $R e_{c f}=3800 . L_{j} / D_{j}=5$. 
FIGURE 5.25: Compilation of wake Strouhal numbers for several combinations of $R e_{c f}$ and $L_{j} / D_{j}$. Average spectral sharpnesses are also shown.

FIGURE 5.26: Strouhal number as a function of $R e_{c f}$ at $V R=4 . L_{j} / D_{j}=5$.

FIGURE 5.27: Wake Strouhal numbers at 3 crossflow Reynolds numbers. $L_{j} / D_{j}=5$.

FIGURE 5.28: Wake Strouhal numbers at 3 crossflow Reynolds numbers. $L_{j} / D_{j}=10$.

FIGURE 5.29: Strouhal number as a function of $R e_{c f}$ at $V R=6.25 . L_{j} / D_{j}=5$.

FIGURE 5.30: Wake Strouhal numbers at $L_{j} / D_{j}=5 \& 10$.
(a) $R e_{c f}=3800$,
(b) $R e_{c f}=7600$.

FIGURE 5.31: Wake Strouhal numbers at several $\delta_{c f} D_{j}$.

FIGURE 5.32: Wake Strouhal numbers from several references.

FIGURE 5.33: Comparison of present wake Strouhal numbers with those of Moussa et al. (1977).

FIGURE 5.34: Wake Strouhal numbers of Moussa et al. for a jet whose supply pipe protrudes into the crossflow.

FIGURE 5.35: Wake Strouhal numbers of wall-mounted circular cylinders as a function of aspect ratio.

FIGURE 5.36: Dependence of $\bar{\delta}_{w} / D_{j}$ on distance from the crossflow wall. (a) $V R=4$, (b) $V R=8$.

FIGURE 5.37: Dependence of $\bar{\theta}_{w} / D_{j}$ on distance from the crossflow wall. (a) $V R=4$, (b) $V R=8$.

FIGURE 5.38: Wake total pressure deficits at $Y / D_{j}=0$. (a) $V R=4$, (b) $V R=8$, (c) CYLINDER. $R e_{c f}=3800 . L_{j} / D_{j}=5$.

FIGURE 5.39: Wake total pressure deficits at $Y / D_{j}=0$. (a) $V R=4$, (b) $V R=8$, (c) CYLINDER. $R e_{c f}=7600 . L_{j} / D_{j}=5$.

FIGURE 5.40: Wake total pressure deficits at $Y / D_{j}=0$. (a) $V R=4$, (b) $V R=8$, (c) CYLINDER. $R e_{c f}=11400 . L_{j} / D_{j}=5$. 
FIGURE 6.1: Sketch of the deflected jet with notation highlighting one vortex of the CVP.

FIGURE 6.2: Experimental results for the nondimensional circulation of one CVP vortex along its trajectory.

FIGURE 6.3: Analytic results for the nondimensional circulation of one CVP vortex along its trajectory.

FIGURE 6.4: Notation for vorticity flux estimate at the jet orifice.

FIGURE 6.5: Idealized model of a jet leading to a counterrotating vortex pair.

(a) Side view, (b) Projection onto a constant $Z$ plane.

FIGURE 6.6: $s_{v 1} / D_{j}$ vs. $V R$. Open circles indicate averages from figures 6.2 and 6.3 references. Solid points show the ranges of disagreement among those results.

FIGURE 6.7: View of deflected jet used to estimate the convective velocity at the point of CVP vortex full-development.

FIGURE 6.8: Convective velocities. Estimates from equation 6.16 and experimental results. Locations of measurements are indicated next to the data.

FIGURE 6.9: Comparison of the predicted circulation of a CVP vortex with the results of others.

FIGURE 7.1: A location of $\zeta$, the $Z$ component of vorticity, is indicated by the arrows.

FIGURE 7.2: Schematic of the total pressure gradient across a plane shear layer.

FIGURE 7.3: Wake Strouhal numbers with five $V R$ regimes indicated. $R e_{c f}=3800$. $L_{j} / D_{j}=5$.

FIGURE 7.4: Typical wake side views. The arrow in each photograph indicates the jet location. (a) $V R=2.1$, (b) $V R=3$, (c) $V R=4$, (d) $V R=5.8$, (e) $V R=6.6$, (f) $V R=8$. $R e_{c f}=3800 . Z_{s w} / D_{j}=0+$.

FIGURE 7.5: Typical views of the near wall, very near wake. (a) $V R=2$, (b) $V R$ $=3$, (c) $V R=4$, (d) $V R=8 . R e_{c f}=3800 . Z_{s w} / D_{j}=0+$. 
FIGURE 7.6: Entrainment trajectories of the separated crossflow boundary layer fluid. (a) $V R=2$, (b) $V R=4$, (c) $V R=8$.

FIGURE 7.7: $S t_{j}$ along the leading edge of a transverse jet. $V R=4 . R e_{c f}=3800$.

FIGURE 7.8: $S t_{j}$ along the potential core of a free jet. $R e_{j}=15200$.

FIGURE 7.9: Decay of temperature in heated transverse and free jets. From Kamotani \& Greber (1972).

FIGURE 7.10: Reacting transverse jet and free jet flame lengths. From Broadwell \& Breidenthal (1984).

FIGURE B.1: Sample nominal crossflow boundary layer profile at $X / D_{j}=0$ showing experimental data and a Blasius profile.

$R e_{c f}=3800 . L_{j} / D_{j}=10$.

FIGURE B.2: Sample nominal jet boundary layer profile at $Z I D_{j}=.013$. Experimental data are shown. $R e_{j}=7600$. 


\section{List of symbols}

$\begin{array}{ll}\text { CVP } & \text { counterrotating vortex pair } \\ \mathrm{N} & \text { node critical point } \\ \mathrm{S} & \text { saddle critical point }\end{array}$

A

$\hat{A}_{j}$

$A R$

$A_{1}$

$C_{p t o t}$

$D_{c}$

$D_{j}$

$\hat{e}_{\theta}, \hat{e}_{z}$

$f_{j}$

$f_{\text {sep }}$

$f_{w}$

$\underline{J} o$

$L_{j}$

$l^{*}$

$\hat{n}$

$P$

$p$

$p_{t c f}$

ptwake

area vector

jet cross sectional area

circular cylinder aspect ratio

CVP vortex cross sectional area at $s_{v 1}$

wake total pressure coefficient, $\frac{p_{t w a k e}-p_{t c f}}{\frac{1}{2} \rho U_{c f}^{2}}$

circular cylinder diameter

jet diameter

polar coordinates unit vectors

local jet frequency

separation event power spectral peak frequency

wake power spectral peak frequency

vorticity flux tensor at a wall, $-\nu(\nabla \vec{\omega})_{0}$

distance from the crossflow wall leading edge to $X=0$

transverse jet global length scale, $\sqrt{\frac{\rho_{j} U_{j}^{2} A_{j}}{\rho_{c f} U_{c f}^{2}}}$

wall-normal unit vector

rms spectral power amplitude

pressure

crossflow total pressure

wake total pressure 
$Q_{1}$

$r, \theta$

$R e_{c f}$

$R e_{j}$

$S t_{j}$

$S t_{\text {sep }}$

$S t_{w}$

$S t_{\theta j}$

$s_{v}$

$s_{\nu 1}$

$U$

$\vec{u}$

$U_{c f}$

$U_{j}$

$u_{r m s}^{\prime}$

$U_{1 \text { conv }}$

V

$V R$

W

$w_{r m s}^{\prime}$

$X$

$X_{s w}$

Y

$Y_{s w}$

$Z$

$Z_{s w}$ volume flow rate through $A_{1}$

polar coordinates

nominal crossflow Reynolds number, $U_{c f} D_{j} / v$ or $U_{c f} D_{c} / v$

nominal jet Reynolds number, $U_{j} D_{j} / v$ or $V R R e_{c f}$

jet Strouhal number, $f_{j} D_{j} / U_{j}$

separation event Strouhal number, $f_{s e p} D_{j} / U_{c f}$

wake Strouhal number, $f_{w} D_{j} / U_{c f}$

separating jet boundary layer Strouhal number, $f_{j} \theta_{j} / U_{j}$

CVP vortex trajectory coordinate, measured from $Z=0$

$s_{v}$ where $\Gamma$ is maximum

velocity in the $X$ direction

velocity vector

nominal crossflow velocity

nominal jet velocity

rms fluctuating velocity in the $X$ direction

convective velocity through $A_{1}$

mean velocity in the $Y$ direction

nominal jet to crossflow velocity ratio, $U_{j} / U_{c f}$

mean velocity in the $Z$ direction

rms fluctuating velocity in the $Z$ direction

crossflow coordinate, measured from the jet

smoke-wire $X$ coordinate

lateral coordinate, measure from the jet

smoke-wire $Y$ coordinate

jet direction, measured from the crossflow wall

smoke-wire $Z$ coordinate 


$\begin{array}{ll}\delta_{c f} & \text { nominal crossflow boundary layer displacement thickness at } X=0 \\ \delta_{j} & \text { nominal jet nozzle boundary layer displacement thickness at } Z=0 \\ \delta_{w} & \text { wake displacement thickness approximation } \\ \bar{\delta}_{w} & \text { space mean wake displacement thickness approximation } \\ \Phi_{j} & \text { vorticity flux from jet nozzle } \\ \Phi_{j, x} & \xi \text { flux from jet nozzle } \\ \Phi_{1} & \text { vorticity flux through } A_{1} \\ \Gamma & \text { CVP vortex circulation } \\ \gamma & \text { nondimensional CVP vortex circulation, } \Gamma /\left(2 U_{c f} D_{j}\right) \\ \Gamma_{1} & \text { maximum CVP vortex circulation } \\ \gamma_{1} & \text { maximum } \gamma, \Gamma_{1} /\left(2 U_{c f} D_{j}\right) \\ \nu & \text { kinematic viscosity } \\ \theta_{c f} & \text { nominal crossflow boundary layer momentum thickness at } X=0 \\ \theta_{j} & \text { nominal jet nozzle boundary layer momentum thickness at } Z=0 \\ \theta_{w} & \text { wake momentum thickness approximation } \\ \bar{\theta}_{w} & \text { space mean wake momentum thickness approximation } \\ \rho & \text { density } \\ \rho_{c f} & \text { crossflow density } \\ \rho_{j} & \text { jet density } \\ \vec{\tau}_{o} & \text { wall shear stress } \\ \vec{\omega} & \text { vorticity vector } \\ \xi & X \text { component of vorticity } \\ \eta & Y \text { component of vorticity } \\ \zeta & \end{array}$




\section{Chapter 1}

\section{Introduction}

The transverse jet, or jet in a crossflow, is widely used in technical applications. Jet injection is used to mix the injected fluid with that of the crossflow into which it is injected. Such mixing applications include dilution jets in gas turbine engines, fuel injection, and waste disposal into the environment. Aerodynamic applications include the exhaust jet/crossflow interaction of V/STOL aircraft. Even natural phenomena, such as volcanic eruptions, may have characteristics of transverse jets.

The transverse jet is also a flow with issues inherent to fundamental turbulence research. Even when turbulent and, by nature, fully three-dimensional, the near field of the transverse jet is dominated by coherent structures. The overall goal directing the present research has been to obtain a better understanding of this structure and its role in turbulent mixing.

Prior investigations of transverse jets at GALCIT have concentrated on the far field (Broadwell \& Breidenthal 1984; Kuzo \& Roshko 1984). By the time the far field is reached, the jet has developed into a counterrotating vortex pair. Broadwell \& Breidenthal analyze the far field vortex pair by considering the jet to be a point source of normal momentum. They also discuss reacting water jet experiments which suggest that transverse jets mix jet and ambient (crossflow or reservoir) fluid more rapidly than free jets do. Kuzo \& Roshko have found welldefined wake vortices in the far field, up to several hundred jet diameters from the 
orifice.

With this backdrop of research in the far field of the transverse jet, it became an interest to investigate the region of the flow close to the orifice of the jet, where most of the jet bending occurs and where the jet/crossflow interaction dynamics are the most complex. Although several kinds of near field structures were investigated, emphasis was placed on the structure and formation of the wake vortices in the course of the present research.

Both the axisymmetric turbulent jet and the laminar crossflow of this study are incompressible air flows of equal densities. Among the important parameters defining the flow are the jet to crossflow velocity ratio and the jet Reynolds number. Here, jet to crossflow velocity ratios of 2 through 10 are investigated, and jet Reynolds numbers are of order $10^{4}$ to $10^{5}$.

\subsection{Fluid mechanical motivation}

One emphasis of fluid mechanics research during the past two decades has been on the role of large-scale vortical structures in mixing of turbulent flows.

One of the fundamental free shear layers is the plane turbulent mixing layer. The dominant role of large-scale coherent structures in plane turbulent mixing layers was realized with a series of experimental investigations in the early 1970s (Brown \& Roshko 1971; Brown \& Roshko 1974; Winant \& Browand 1974; Roshko 1976; Dimotakis \& Brown 1976). These references document well the significance of the large structures on turbulent entrainment and mixing. It was discovered that the entrainment processes of plane mixing layers at Reynolds numbers up to order $10^{5}$ and $10^{6}$ (based on the high speed stream velocity and kinematic viscosity, and downstream distance) are dominated by vortical structures on the 
scale of the mixing layer width. Mixing at the molecular scale is then realized by the smaller-scale turbulence and diffusion. These processes are secondary to the large-scale vortical structures, the role of which is to entrain nonturbulent and irrotational fluid into the turbulent region of the shear layer.

At about the same time, structure in the turbulent free jet had also been observed. Crow \& Champagne (1971) observed large-scale "vortex puffs" near the end of the potential core/mixing layer region of a turbulent jet. These structures were found to have a characteristic frequency of formation. Yule (1978) also found coherent eddies in the near field of a round turbulent jet. Furthermore, it was found that the self similar far field region of the axisymmetric free jet is also dominated by large scale structure dynamics (Tso, Kovasnay \& Hussain 1981; Dimotakis, Miake-Lye \& Papantoniou 1983; Dahm \& Dimotakis 1985).

Although more complicated than the plane mixing layer and the free jet, the transverse jet is also a canonical flow. It follows, then, that both the presence and role of coherent structures of the transverse jet should also be investigated.

\subsection{A review of transverse jet research}

The earliest reference to the jet into a crossflow problem may be Dobson (1919). The purpose of that report, however, was not to specifically study the transverse jet. Instead, smoke trails from a factory chimney in a crosswind were used to estimate the turbulence "eddy conductivity" in the atmosphere. Bosenquet \& Pearson (1936) studied the transverse jet with regard to the spread of emissions from chimneys.

As mentioned earlier, the far field of the transverse jet is dominated by a counterrotating vortex pair. A drawing of the jet with this structure is shown in 
figure 1.1. The vortex pair has also been referred to as the bound vortices of the jet, in the sense that they comprise the main portion of the deflected jet sufficiently far from the orifice. Somewhat misleading are references to these counterrotating vortices as "wake vortices." For the present purposes, such a label is not used. Wake vortices, as they are referred to here, are distinct from the vortex pair of the jet and are addressed in detail later.

An early reference which shows that the jet should form a counterrotating vortex pair as it bends is Scorer (1958). The analysis of Scorer is based on the jet orifice supplying an impulsive source. Scorer also shows photographs, now familiar, of smokestacks with jets (and plumes) developing or bifurcating into a pair of structures aligned with the jet trajectory. More recently, Broadwell \& Breidenthal (1984) analyze the far field by considering the primary far field structure of the transverse jet to be a counterrotating vortex pair.

There are numerous experimental studies of the counterrotating vortex pair structure of the jet. A partial list, whether strictly in the far field or not, is the following: Margason \& Fearn (1969), Kamotani \& Greber (1972), Fearn \& Weston (1974), and Moussa, Trischka \& Eskanazi (1977). Margason \& Fearn also include a list of references on V/STOL applications. The experiments generally consider only the time-mean properties of the counterrotating vortices. Whether this mean structure has superimposed on it other (time-dependent) structure had not been addressed. Experiments by Keffer \& Baines (1963) and Pratte \& Baines (1967) address jet trajectories, jet profiles, and similarity considerations.

Since experiments have shown that the vortex pair dominates the developed jet, many transverse jet models of both the near and far fields use the counterrotating vortex pair as their basis (Durando 1971; Fearn \& Weston; Le Grieves' 1978; Broadwell \& Breidenthal; Nunn 1985; Karagozian 1986). Of these, only the 
models of Broadwell \& Breidenthal and Karagozian do not require some sort of experimental data as input.

Sykes, Lewellen \& Parker (1986) attempt to compute the jet into a crossflow using the 3-D Reynolds-averaged, Navier-Stokes equations. The formation of the counterrotating vortices is seen. Lagrangian information for the flow near the orifice is shown using particle trajectories, useful in gaining insight into the early development of the jet, as modelled.

Coehlo \& Hunt (1989) investigate three different time-dependent vortexsheet models. Their results indicate that a 3-D model with an imposed entrainment velocity is required to properly represent the near field dynamics (for large jet to crossflow velocity ratios), in particular to have the jet deflect in the direction of the crossflow. In the process of applying their three models, new issues such as the need to address precisely the mechanism by which the jet bends and flow nonuniformities within the nozzle itself are raised.

One issue relevant to the experimental study and the modelling of transverse jets is whether this flow is truly a free shear flow. In particular, what is the effect of the wall with which the jet may be mounted flush? In most experiments, and apparently in all computations (including the references listed above), the precise effect of this wall, particularly the boundary layer on it, is not considered. Andreopoulos (1985) does, however, consider the boundary layer on the wall from which the jet issues but only for very low jet to crossflow velocity ratios of less than one. Foss (1980) also considers wall effects by studying the near field wall surface topology. Critical point (node and saddle) constraints of Hunt et al. (1978) along with flow visualization are used to describe the topology.

The structure of the transverse jet very near the orifice has not been studied extensively. One exception is the work of Moussa et al.; they address, in particular, 
vorticity flux and dynamics issues in this interaction region. Andreopoulos notes the presence of ring-like vortical structures early in the jet development for velocity ratios in excess of about 3 .

The transverse jet also has a wake. Periodic motion in the wake (the region of the flow between the deflected jet and the wall from which the jet issues) was detected as early as 1968 (McAllister 1968; Reilly 1968). McMahon et al. (1971) and Wu, Vakili \& Yu (1988) have measured and observed coherent (vortical) fluctuations in the near wake. Kuzo \& Roshko (1984) used dye to most clearly visualize the wake vortices, and they report on the persistence of wake vortices to several hundred jet diameters downstream. All investigators have viewed the wakes of transverse jets as similar to the wakes created by vortex shedding from solid cylinders.

In general, most of the transverse jet references mentioned here deal with jet to crossflow velocity ratios similar to the ones studied here.

\subsection{What is the near field?}

Several criteria are available to separate the transverse jet into near and far fields.

Whether the near or far field is addressed can be a function of what issue is being studied. In this sense, observing and quantifying developed structures such as the counterrotating vortices or the wake vortices is a far field study. Conversely, it is a near field study if the goal is to understand the initial development and formation of the structures. The near field is where the interaction between the crossflow and jet is most intense; the three-dimensional development of the jet is most dynamic there.

A global length scale of the transverse jet flow is given by $l^{*}$ (Broadwell \& 
Breidenthal 1984). This length scale is given by

$$
l^{*}=\sqrt{\frac{\rho_{j} U_{j}^{2} A_{j}}{\rho_{c f} U_{c f}^{2}}} .
$$

For jets and crossflows of equal densities, this is approximately given by the product of the jet to crossflow velocity ratio and the jet diameter. The near field can also be defined as the portion of the flow within $l^{*}$ of the jet orifice. This essentially corresponds to the distance within which the jet has done a significant portion of its bending.

Here, approximately the first ten jet diameters of the flow are being studied. This includes a range of $l^{*}$ from approximately one through five, depending on the jet to crossflow velocity ratio.

\subsection{A preview}

Although many observations and measurements have been made, and many models have been constructed, there is still no complete understanding of the structure in the near field of the transverse jet. This research addresses this issue. For reasons which will become clear, the characteristics and formation of the wake receive the most emphasis.

The presentation of this research in what follows is generally in the order in which the research was conducted. First, flow visualization displays the four dominant near field vortical structures. These are the jet shear layer vortices, the nascent far field vortex pair, the near wall horseshoe vortices, and a system of vortices in the wake. As a preview, sketches of the four types of near field structure are shown in figure 1.2. As will be seen, the wake structures are most striking. They, however, have received relatively little detailed study in the literature. Most importantly, the wake is intriguing because there is no solid bluff body obstacle in 
the flow. The question raised, therefore, is whether comparisons to a solid cylinder wake are valid. The issue of vorticity generation then enters. Since coherent structures are essentially concentrated regions of vorticity, understanding where the vorticity comes from is an essential first step to understanding the structures. Additional flow visualization and measurements are then used to determine the origin and formation mechanism of the wake structures. The near field development of the counterrotating vortex pair is also addressed, continuing with the theme of sources of vorticity for coherent structures. The possible implications of the new results on mixing are also addressed. 


\section{Chapter 2}

\section{Experimental set-up}

\subsection{The crossflow}

The crossflow was provided by GALCIT's 20 " by 20 " open-return low speed wind tunnel. Figure 2.1 shows a schematic of the wind tunnel. Cimbala (1984) improved the test section turbulence level by adding several screens in the settling chamber and installing a new test section.

Further modifications to the tunnel contraction section were necessary. The use of the tunnel in this research was somewhat unique, in that the transverse jet set-up required the use of the flow near one of the walls of the test section. Smoke-wire flow visualization and hot-wire traverses indicated that the flow close to and near the center of each wall of the test section was unacceptably poor (turbulent). The source of these patches of turbulent flow was eventually traced back to the extreme curvature of the concave portion of the contraction section. Apparently, separation at this initial region of the contraction contaminated the flow in the test section. Wood fillet members with aluminum sheets were installed at each wall to smoothen that portion of the contraction. The flow in the test section was then sufficiently laminar everywhere. Figure 2.2 shows the before and after contraction contours, drawn approximately to scale.

The flow in the test section was found to be quite sensitive to the room 
conditions. In particular, care was taken to remove any unnecessary perturbations to the tunnel inlet while experiments were run. All ventilation outlets in the vicinity of the tunnel were turned off or closed during runs. For instance, a vent open to the outdoors near the inlet caused a swirling flow in the test section due to the imposed temperature/density gradients. The direction of swirl was dependent on whether it was warmer or cooler outdoors than indoors. Also, doors to the room were left open during runs to prevent perturbations caused by their opening and closing for normal traffic.

The majority of experiments were performed at three nominal crossflow velocities: $U_{c f}=1.5,3$, and $4.5 \mathrm{~m} / \mathrm{s}$. The velocity varied by less than $2 \%$ across the span of the test section. The turbulence intensity levels $\left(u_{r m s}^{\prime} / U_{c f}\right)$ were about $.25 \%, .2 \%$, and $.2 \%$ for $U_{c f}=1.5,3$ and $4.5 \mathrm{~m} / \mathrm{s}$, respectively.

To provide better control of the boundary layer on the wall from which the jet issues, a false side wall was used. This false side wall is also referred to as the crossflow wall, and the boundary layer on this wall is referred to as the crossflow boundary layer. Figure 2.3 shows a detailed view of the test section. The false side wall has an elliptical leading edge with a major to minor axis ratio of six. Without modification, the streamwise pressure drop in the channel between the false side wall and the nearest wall of the tunnel is larger than that of the freestream. Therefore, the flow around the leading edge separates and contaminates the crossflow wall boundary layer. To remedy this, control screens of appropriate solidity were placed in the freestream portion at the end of the test section (refer to figure 2.3). As indicated by table 2.1, the number of screens used and their solidity were a function of $U_{c f}$.

The distance from the leading edge of the crossflow wall to the jet orifice is given by $L_{j} . L_{j} / D_{j}=5$ and 10 were used in the experiments. Table 2.2 shows the 
nondimensionalized nominal crossflow boundary layer displacement and momentum thicknesses at $X / D_{j}=0$ for each of the three nominal crossflow velocities with $L_{j} / D_{j}=5$ and 10 . Measured values and those calculated assuming a Blasius boundary layer are included. (Refer to appendix B for how $\delta_{c f}$ was determined from measurements). In addition, turbulence intensity levels in the boundary layer at $Z$ where $U=U_{c f} / 2$ are included. The resulting undisturbed crossflow boundary layer is laminar in all cases.

\subsection{The jet}

The jet was supplied by a 1.5 " diameter nozzle, which was mounted flush with the crossflow wall, i.e, the false side wall. The jet set-up, including the nozzle, flow management section, settling chamber, and blower, is shown in figure 2.4.

The jet is powered by a Cincinnati Model HPA radial vane centrifugal blower. The blower is fitted with a Pacific Scientific Model SRF3640-4576-7-56C permanent magnet $\mathrm{DC}$ motor $(1 \mathrm{HP}, 3450 \mathrm{rpm}, 90 \mathrm{VDC})$. The jet velocity is set with a $\mathrm{B}$ \& B Logic II Model LGC1P1 motor speed controller. The blower sizing was based on achieving jet velocities to $50 \mathrm{~m} / \mathrm{s}$.

Modifications to the blower were required to reduce or eliminate significant fluctuations in the jet which matched the blower vane passage frequencies. Placing a flat cover on the hub of the fan where the radial vanes meet helped significantly. In addition, the blower housing was widened to effectively spoil the flow coming off of the vanes. A settling chamber with 1 " foam lining was placed between the blower and the flow management section of the jet supply after trial and error tests revealed that jet turbulence intensities were reduced.

Wind tunnel design techniques were used in the design of the flow manage- 
ment section and nozzle (Morel 1975; Loerke \& Nagib 1976; Mehta \& Bradshaw 1979; Nagib, Marion \& Tan-atichat 1984; Coles 1985; Dimotakis 1985). The flow management section contains two screens of $70.2 \%$ open area (18 Mesh, .009" wire diameter). A 1/8" cell diameter honeycomb section is placed just upstream of the 2nd screen, as recommended by Loerke \& Nagib. Coles recommended a large honeycomb length to cell diameter ratio but not too large, for transition to turbulence within the cells should be avoided. Here this ratio is 96 , and the largest "pipe" Reynolds number encountered (for $U_{j}=50 \mathrm{~m} / \mathrm{s}$ ) is therefore 1200 , well below the transition Reynolds number for pipe flow.

A matched cubic contour was selected for the nozzle contraction. The point of inflection for the contour is at $60 \%$ from the inlet. The nozzle area contraction ratio is nine. The nozzle is designed to prevent boundary layer separation at its inlet, to prevent the formation of Goertler vortices on its concave portion, and to produce a nearly top hat velocity profile at its exit. In particular, Liepmann (1945) shows that transition to Goertler vortices occurs on concave walls when the parameter

$$
\frac{U_{\infty} \theta}{\nu} \sqrt{\frac{\theta}{R}}
$$

is greater than 6 (when the freestream turbulence level is at .3\%). $U_{\infty}$ is the local freestream velocity, $\theta$ is the boundary layer momentum thickness, and $R$ is the local radius of curvature. Assuming laminar boundary layer development beginning at the 2nd screen, 1-D flow within the nozzle, and by applying Thwaites method to calculate the boundary layer thickness on the concave portion of the nozzle, this parameter is below 2 for all jet velocities of interest in these experiments.

The nozzle was made out of Devcon aluminum liquid (F-2), an epoxy compound. First an aluminum mandrel was machined with its outer surface matching the desired contour of the nozzle. To form the nozzle, the Devcon aluminum liquid 
was poured into a mold comprised of the mandrel and an outer form of arbitrary shape. After the aluminum liquid cured, the mandrel was pulled out, revealing the desired contoured inside surface of the nozzle.

A jet exit velocity profile at a sample $U_{j}$ is shown in appendix B (figure B.2). Table 2.3 shows jet exit turbulence intensity levels $\left(w_{r m s}^{\prime} / U_{j}\right)$ and measured nondimensional boundary layer displacement and momentum thicknesses at the nozzle exit for selected $U_{j}$. (Refer to appendix B for how $\delta_{j}$ and $\theta_{j}$ were determined from measurements.)

\subsection{Flow visualization}

The smoke-wire flow visualization technique (Corke et al. 1977) was used extensively. This technique is also described by Cimbala (1984). The smoke-wire technique produces closely spaced streaklines. A schematic of the smoke-wire setup, as employed here, is shown in figure 2.5. Since a closed electrical circuit is necessary to operate the smoke-wire, a reasonably non-intrusive and easily mobile set-up is not a trivial matter. Such requirements were met here by attaching the top end of the .005 " stainless steel wire through a hypodermic needle and tube which was mounted on a spring-loaded movable support. A "floating" weight was attached to the bottom end of the wire to keep the wire taut. Thus the whole smoke-wire unit could be traversed by simply moving the top mounting piece. Smoke oil (supplied by Flow Visualization Systems) is injected through the top tube. A pressurized line forces the oil through the hypodermic needle onto the wire. Three drops of oil were used per visualization/photograph. For simplicity of operation, the smoke-wire was always vertical. Depending on the view required, therefore, the jet was either mounted horizontally through one of the side walls 
or vertically through the top wall of the tunnel. The false side wall had thin slots (sealed by foam gaskets) through which the hypodermic needle could fit. The smoke-wire could then be traversed downstream of the wake when the jet issued from the top of the test section.

In some cases, the jet flow was visualized by seeding the jet supply with cigarette smoke. Ten cigarettes were simply placed near the inlet of the centrifugal blower. The inflow of air kept the cigarettes burning.

Photographs were taken using a Pentax ME-Super $35 \mathrm{~mm}$ camera with a motor drive. The lighting was provided by a General Radio Model 1540 Stroboscope. The best results were achieved with the strobe directed vertically almost in line with the plane of smoke streaklines. (Refer to figure 2.5). The proper camera, strobe, and smoke-wire timing was accomplished by a controller designed and constructed by Flow Visualizaton Systems. At the velocities of interest, the smoke-wire was heated for approximately one second for each visualization. A Video Logic CDR360 video camera was used for video recordings. The strobe was synchronized with the framing rate of the video camera (at $30 \mathrm{~Hz}$.). Additionally, flood lighting was used for the video recordings.

Either Kodak Tri-X (ASA 400) or Kodak TMAX (ASA 400) film, developed normally, was used for the still photographs. Prints were made on Ilford glossy polycontrast paper. A contrast filter of two was used for printing.

All photographs shown in subsequent chapters (except figure 3.5) show the jet issuing either at the viewer or from the bottom of the photograph. The crossflow is left to right in all cases. Note that showing the jet issuing from bottom to top is a convention adopted here for the presentation of the photographs; the experimental set-up, as stated previously, actually had the jet issuing from top to bottom in such cases. Figure 2.6 shows two typical smoke-wire/jet orientations 
for the photographs. In the top sketch of figure 2.6 the streaklines of smoke begin in a $Y / D_{j}=$ constant plane, while in the bottom sketch the streaklines of smoke begin in a $Z / D_{j}=$ constant plane.

\subsection{Measurement apparatus}

In order to traverse measurement probes in the three-dimensional flow field, an $\mathrm{XYZ}$ traversing system was purchased and installed at the wind tunnel. VelmexUnislide slide assemblies with travel lengths of 22", 8", and 15" in the $X, Y$, and $Z$ directions, respectively, were used. Each axis was fitted with a Compumotor Series LE microstepping low noise motor and was computer-controlled with a Compumotor PC-23 three axis indexer. The indexer was installed in a Everex System 1800 (AT compatible) computer.

Single-wire hot-wires (either TSI 1210-T1.5 or TSI 2160-T1.5) were used for velocity and spectral measurements. A Matilda meter constant temperature anemometer (in-house built) was used with the hot-wires. Power spectra were obtained from the hot-wire output using an HP $3582 \mathrm{~A}$ real time spectrum analyzer. An HP 3403C true rms voltmeter was used to obtain turbulence fluctuation levels. Appendix B includes measurement details concerning the spectral measurements.

Crossflow velocities were measured, and hot-wire calibrations were performed with a United Sensor pitot-static tube. Total pressures were measured by a United Sensor 1/4" diameter Venturi (type KC) Kiel probe. These probes were connected to a Datametrics Barocel pressure sensor (10 torr range) and a Datametrics Model 1173 Barocel Electronic manometer.

Averages of signals (both pressures and velocities) were obtained over ten seconds with a HP 5326C True rms voltmeter. 


\subsection{Nominal vs. actual velocities}

The crossflow velocity $\left(U_{c f}\right)$, jet velocity $\left(U_{j}\right)$, and the jet to crossflow velocity ratio $(V R)$ values given in this thesis are nominal values. That is, they are the jet conditions with no crossflow and crossflow conditions with no jet. Turning on either the crossflow or jet changes the actual velocity of the other. Therefore the nominal and actual values are not the same.

Table 2.4 compares directly the nominal velocity ratios with the actual velocity ratios. The actual velocity ratios are generally somewhat larger than the nominal values. This is because of the effects of the crossflow on the jet and vice versa. For instance, since the wind tunnel is of the suction variety, the pressure in the test section is slightly below atmospheric. Therefore, for the same speed control setting for the jet, the actual jet velocity increases slightly with the crossflow on. The presence of the jet in the crossflow essentially results in a blockage effect. The actual crossflow velocities, measured $15 D_{j}$ upstream of the orifice, are thus somewhat lower. These two effects each contribute to actual velocity ratios which are larger than the nominal ones. 


\section{Chapter 3}

\section{Near field flow visualization}

To commence with the results of this research, a collection of photographs showing the near field of the transverse jet is presented in this chapter. The photographs reveal that the near field is rich with vortical structure.

The terms "structure" and "vortex" are used equivalently here. Therefore "vortical structure" is, strictly speaking, redundant but is used for emphasis on occasion. A feature in a photograph is considered a structure or vortex if it is relatively well-organized and if it appears rotational in nature. Although this leaves the determination open to some interpretation, what is or isn't a vortex is quite clear in most cases.

The near field portion of this flow is divided into three regions: the deflected jet itself, the flow near the crossflow wall, and the wake region. Although the vortices in each of these regions interact with those in the others, this division is a matter of convenience for presenting the flow visualization results.

Among these three regions, four dominant near field vortical structures have been identified. The sketch in figure 1.2 indicates each of the four structures. Two of them, the distorted shear layer vortices at the circumference of the deflected jet and the inception of the counterrotating vortex pair, which eventually dominates the far field jet structure, are inherent to the deflected jet. At the crossflow wall, a system of horseshoe vortices is seen, as is additional near wall structure at the lee 
side of the jet. In the wake region of the jet, a system of vortices tilted somewhat with respect to the initial jet direction is observed.

The smoke-wire flow visualization technique, described in section 2.3 , was used for most of the photographs. In some cases, the jet supply was seeded with cigarette smoke instead. Strobe lighting was used except where noted. Additionally, in nearly all cases (except for those otherwise noted) general lighting was used, as opposed to a "sheet" of light.

Several conventions are adopted for the presentation of the photographs; these are reviewed here. In many cases, the dependence of the structures on $V R$ is shown by including examples with $V R=2,4,6,8 \& 10$ for each type of structure. The structures discussed are at least qualitatively independent of $R e_{c f}$, unless otherwise noted. For the majority of the photographs shown, $R e_{c f}=3800$, and, in all cases, $L_{j} / D_{j}=5$. Generally, the crossflow is from left to right, and the jet issues either from the bottom of the field of view or at the viewer ${ }^{1}$. The smoke-wire is upstream ${ }^{2}$ of the viewing area unless indicated otherwise. Each figure caption gives the plane in which the smoke streaklines originated, i.e., the plane of the smoke-wire.

\subsection{Structure of the deflected jet}

\subsubsection{Distorted jet shear layer vortices}

One characteristic feature of the deflected jet is its vortex ring-like structure, resulting from the Kelvin-Helmholtz instability of the initially annular-like shear

\footnotetext{
${ }^{1}$ For the cases where the jet issues "at the viewer", the camera is not necessarily coincident with the $Z$ axis. Therefore using the crossflow wall to visually gauge the $X$ position of flow features can be slightly inaccurate. Refer to table B.2 for camera locations for such photographs.

2 "Upstream" and "downstream" are used in relation to the crossflow direction.
} 
layer separating from the edge of the jet orifice. In common with initial portions of free jets (Freymuth 1966; Becker \& Massaro 1968; Gutmark \& Ho 1983), this instability also makes the vortex ring a basic structure of the transverse jet, at least in its initial development. Because of the inherent three-dimensionality of transverse jets, the ring-like structure here is relatively more distorted than in a simple free jet.

Figure 3.1 shows leading edges of the jet distorted shear layers at $R e_{\mathrm{c} f}=3800$ for the five velocity ratios. The approaching streaklines, visualized with a smokewire upstream and in the $Y=0$ plane, are entrained into the leading edge shear layer of the jet. As expected, the smaller the $V R$, the larger the shear layer curvature. As a result of axial flow along the cores of the distorted vortex rings, the smoke is sometimes seen coming out of the $Y=0$ plane. Such axial flow is faintly seen close to the orifice in figures $3.1 \mathrm{a} \& \mathrm{~b}$. Figure 3.2 shows a close-up of a leading edge shear layer and shows more clearly the accompanying out of plane flow.

Since the out of plane flow in figure 3.2 is along the cores of distorted vortex rings, the direction of tilt for these structures is indicated. The portions of the rings visualized in figure 3.2 are those closest to the leading edge of the jet and tilt clockwise. The rotation of the remaining portion of each ring that is closer to the trailing edge of the jet is not clear from the present results. Furthermore, the direction of tilt of the shear layer vortices at higher velocity ratios is not clear.

Figure 3.3 shows another view of crossflow fluid entrainment into the shear layer of the jet. In figure 3.3a, the smoke streaklines begin in the $Z / D_{j}=.75$ plane. The flow approaching the $V R=2$ jet is entrained by the upstream side of the jet, as is evident from the four arch-like structures there. These arch-like structures are the upstream portions of four vortex rings, such as those visualized in side 
view in figure 3.2 . Figure $3.3 \mathrm{~b}$ shows entrainment into a relatively strong jet (VR $=10)$. Streaklines from a smoke-wire at $Z / D_{j}=.5$ indicate flow radially inward toward the jet core at all angular locations around the jet. Entrainment is not only important for the mixing process, but is also important for the dynamics of the deflecting jet. The analyses of Coehlo \& Hunt (1989) suggest that entrainment into the shear layer region of the jet is the primary mechanism for deflecting the jet into the direction of the crossflow.

The initial trajectories of transverse jets are visualized in figure 3.4 by seeding the jet supply with cigarette smoke. As usual, general lighting (not a "sheet" of light) was used for these photographs. Even so, the leading edge of the jet shear layer roll-up is again visible as is, in some cases, its trailing edge. The effects of $V R$ on the curvature of the jet are clearly seen again.

Characteristically, the leading and trailing edge structures are seen to collide, indicating the end of the potential core of the jet. Among the photographs in figure 3.4, this is seen for $3.4 a, b, c \&$, faintly, in d. A more diffuse and thicker jet body, still with structure, then results beyond the potential core/shear layer region. Correspondingly, a transition in the leading edge of the deflected jet from a relatively laminar to turbulent appearance occurs. This is most evident in figures 3.1a, b \& c near where the potential cores are likely to end. Such a transition is also observed in free jets of comparable $R e_{j}$. For instance, Crow and Champagne (1971) observed large scale vortex "puffs" near the ends of potential cores of free jets, and Yule (1978) observed less orderly but "strong, large eddies in the fully developed turbulent regions" of free jets. 


\subsubsection{Counterrotating vortex pair}

As is mentioned in the introduction and shown in figure 1.1, the transverse jet forms a pair of counterrotating vortices which dominate the far field. The vortex pair remains roughly aligned with the local direction of the jet. In the far field, the pair becomes nearly aligned with the crossflow. Present results indicate that the counterrotating vortex pair begins forming quite early. For example, figure 3.5 shows a nearly planar slice of the flow at $X / D_{j}=1$. (A "sheet" of light was used in this case.) Smoke begins in the crossflow boundary layer, is entrained away from the wall at the lee side of the jet, and then labels a structure which suggests a counterrotating vortex pair. The sketch accompanying the photograph shows that such a cross section is expected. In the photograph, the smoke outside the outlined area of the jet cross section is in the wake of the jet and is therefore not part of the main portion of the jet. Such a vortex pair structure is observed for the complete range of $V R$ and $R e_{c f}$.

Streaklines originating in constant $Y$ planes which are not directly entrained into the jet shear layer also indicate the presence of a counterrotating vortex pair. Consider figure 3.6, for example, where the smoke-wire is upstream of the jet in the plane defined by $Y / D_{j}=1$. The approaching streaklines wrap around the jet, which is outlined by the dashed lines drawn on the photograph. On the aft side of the jet, they flow toward the centerplane and spiral away from the crossflow wall. Such a flow pattern coincides well with the expected flow about one vortex of the pair. Some of the smoke is entrained by the jet, while the remainder continues into the wake. The sketch in figure 3.6 helps show this three-dimensional flow pattern. Video recordings and observing the experiments in progress show this flow pattern clearly. 
Figure 3.7 shows the flow around the jet with the smoke streaklines originating in three different constant $Z$ planes for each of the five velocity ratios. Very close to the wall (but outside the crossflow boundary layer), the cross section of the jet is nearly circular. The cross section grows and distorts with increasing $Z_{s w} / D_{j}$ in each case. In some photographs, the early portion of a wake is visible just downstream of the jet. Some of the photographs in figure 3.7 (especially $3.7 \mathrm{c}$ center, $3.7 \mathrm{~d}$ center, and $3.7 \mathrm{e}$ center) clearly show evidence of a counterrotating vortex pair in the cross section of the jet. That the cross section of the jet deforms from its initially circular shape into a distorted oval, or kidney-like, shape during its development has been observed previously (Kamotani \& Greber 1972; Moussa, Trischka \& Eskanazi 1977; Coehlo \& Hunt).

\subsection{Structure at the crossflow wall}

\subsubsection{Horseshoe vortices and more}

As an obstacle to the crossflow, the jet produces an adverse pressure gradient just ahead of it at the crossflow wall. Because of the adverse pressure gradient, the approaching boundary layer separates and forms a system of horseshoe vortices. A somewhat similar system of vortices is commonly observed in the near wall flow about wall-mounted solid obstacles (Baker 1980; Mason \& Morton 1987; Thomas 1987).

In figure 3.8, smoke begins in the crossflow boundary layer and labels horseshoe vortices which wrap around the base of the jet. Such is the case for each $V R$. For comparison, figure 3.9 shows a horseshoe vortex system for the flow around a wall-mounted circular cylinder of $A R=6$, at the same $R e_{c f}$ of 3800 . The upstream portions of the horseshoe vortices are visually similar among the transverse 
jets and cylinder, but such is not the case for the downstream portions (legs) of these structures. As is evident from the photographs, the near wall structure in the very near wake (the portion of the wake within a couple of jet diameters of $X$ $=0)$ strongly depends on $V R$. Note also the difference in the very near wake of the cylinder, compared to those for the transverse jets. Because of these differences in the very near wakes, the fate of the horseshoe legs, extending into this region, is affected.

The variety of structure at the aft side of the jet is relevant to the development of wakes of transverse jets. This near wall, very near wake region and its importance to the wake vortices is discussed in detail in chapter 5 .

Cross sections of horseshoe vortices are shown in figures $3.10 \mathrm{a}$ and $\mathrm{b}$. In both cases, two horseshoes whose vorticity is the sign of the crossflow boundary layer vorticity are seen just ahead of the jet.

Generally, one to three horseshoe vortices with vorticity of the sign of the crossflow boundary layer were observed. It is not conclusive from the present results whether or not there is a dependence of the number of horseshoe vortices on either $V R$ or $R e_{c f}$. Owing to the nature of such three-dimensional separations, slight changes in $Z_{s w} / D_{j}$ can influence the number of horseshoe vortices visualized. Furthermore, video recordings show that these vortices are unsteady.

Photographs and videos do suggest, however, that the extent of the horseshoe separation region ahead of a wall-mounted circular cylinder is greater than it is ahead of transverse jets. Additionally, within the limits and uncertainty associated with comparing horseshoes from photograph to photograph, the distance from the lip of the jet orifice to the horseshoe vortices tends to decrease with increasing velocity ratio. Recently, Krothapalli et al. (1990) found such a trend for horseshoe vortices around rectangular jets above $V R=5$. The present results coincide with 
the mean crossflow wall pressure measurements of Fearn \& Weston (1975). They found the extent of the adverse pressure gradient region ahead of the jet to decrease with increasing $V R$. It follows that the horseshoe vortices, formed as a result of the adverse pressure gradient, should move closer to the jet with increasing $V R$.

\subsection{Wake structure}

The wake of the transverse jet is dominated by vortices which extend from the crossflow wall to the deflected jet. Refer again to figure 1.2, which shows a sketch of the wake vortices. The wake structures are generally inclined slightly, in the opposite direction to that of the jet trajectory. Structures in wakes of transverse jets have been observed and/or measured previously on several occasions, and they have been observed up to several hundred jet diameters downstream of the orifice (Kuzo \& Roshko 1984). A complete list of wake references is given in chapter 5.

The wake of the transverse jet, however, has not been studied extensively nor in detail. In particular, the mechanism of wake formation has not been investigated. Generally, the wake vortices of transverse jets have been considered analogous to the shed vortices in wakes of solid circular cylinders. Although the jet is an obstacle to the crossflow, it is not a solid obstacle. What are the effects of not having a solid obstacle? This issue, among others dealing with the wake, is addressed later in chapter 5. For now, photographs showing the general characteristics of the wake are presented.

A wake with vortices exists for all velocity ratios and crossflow Reynolds numbers studied, but, as will be seen, its appearance varies with $V R$.

Side views of the wake vortices are shown in figure 3.11 for the five velocity ratios. In these photographs, the smoke-wire is placed such that essentially only 
the wake vortices are visualized. In these cases, this is accomplished by placing the smoke-wire upstream of the jet so that the smoke streaklines begin parallel to and just above the crossflow wall $\left(Z_{s w} / D_{j}=0+\right)$. To orient the viewer, the dashed lines drawn on these photographs mark an outer boundary of the deflected jet, as deduced from the smoke boundaries in figure 3.4. Figure 3.11a shows that the wake structures are not well-defined for $V R=2$. At higher velocity ratios, however, the vortices are more clearly seen. Note that in some photographs, the trailing edge structure of the jet is faintly visible. The photographs shown here are exemplary of the wake structure for each $V R$.

The wake vortices entrain irrotational crossflow fluid, as is evident in figure 3.12. In each of these photographs, smoke begins upstream of the jet in a $Y \neq 0$ plane. The "sheet" of smoke passes the jet and is then entrained into the wake region, visualizing the wake structures. Again, the wake vortices are not as clear for $V R=2$ as they are at higher velocity ratios. The wake vortices for $V R=8$ $\& 10$ are more striking when the smoke-wire is placed just downstream of the jet and within the wake. See, for example, figure 3.13.

To study the wake further, the smoke-wire was placed in the wake as far downstream as $X_{s w} / D_{j}=10$. The primary purpose was to check the integrating effect of the velocity field on the smoke particles (Cimbala 1984); the appearance of the wake structures was not significantly affected by changes in the downstream location of the smoke-wire (within $10 D_{j}$ ). In the process, it was found that at certain velocity ratios, a region of recirculation or reverse flow immediately downstream of the jet at the crossflow wall exists. When the smoke-wire was positioned within the wake at $X / D_{j}=2$ and $Y / D_{j}=0$, some smoke would flow upstream near the crossflow wall for $V R=2,8 \& 10$. Figure 3.14 shows these results and the dependence of the reverse flow on $V R$. For $V R=2,8 \& 10$, note the reverse 
flow, as indicated by the arrows. For $V R=4 \& 6$, no reverse flow is seen. This dependence on $V R$ exists for the three crossflow Reynolds numbers studied. Video recordings confirm this, and they also show that the fluid in the reverse flow regions rotates clockwise, as is expected. When it exists, the reverse flow is most clearly visualized when $Y_{s w} / D_{j}=0$.

The various side view photographs of the wake structures show that their appearances vary with $V R$, beyond the expected lengthening of the wake vortices as the jet is deflected further from the crossflow wall at higher velocity ratios. The following comments and trends prevail from the study of all the photographs taken at each $V R$. Since the photographs shown here are typical for each $V R$, the comments apply generally. The wake for $V R=2$ is best described as only having puff-like structures rather than well-organized vortices (figure 3.11a). These wake structures are not clearly defined. At $V R=4$, the wake structures are much more clearly defined as vortices (figures $3.11 \mathrm{~b} \& 3.12 \mathrm{~b}$ ). They are thin relative to $V R$ $=2$ wake structures but are thick relative to those at larger velocity ratios. Their thickness is also fairly uniform along their span from the crossflow wall to the jet. Flow visualization for $V R=6$ indicates that the wake structures are similar to those at $V R=4$ (figures $3.11 \mathrm{c} \& 3.12 \mathrm{c}$ ). However, thinner and distorted structures are more prevalent. Also, the wake structures appear to get thinner along their span at the ends closer to the jet. As the velocity ratio is increased further, changes in the wake structures become more apparent. See, for example, figures 3.11d, 3.11e, 3.12d, 3.12e, 3.13, 3.14d \& 3.14e. Here, at $V R=8 \& 10$, the wake structures are characteristically thin and strand-like, and less ordered. Thicker structures are only intermittently observed. At these larger velocity ratios, most of the smoke, which originated in the crossflow boundary layer, stays within a couple of jet diameters of the wall as somewhat clumpy structures (figures $3.11 \mathrm{~d}$ 
\& 3.11e, in particular). This near wall portic of the wake is connected to the jet by the strand-like structures. This situation is more extreme for $V R=10$ than $V R=8$.

To summarize, the side views of the wake indicate that there is a change in the wakes character from poorly defined puff-like structures at $V R=2$ to very well-organized wake vortices at $V R=4$. Then, from $V R=6$ to $V R=10$ there is a change from wakes whose structures are fairly uniform along their spans to wakes which are split into a region of dense, clumply structures near the crossflow wall and a region of very thin, strand-like structures extending to the deflected jet. Such dependence on $V R$ is discussed more completely in connection with other results and the wake formation in chapter 7.

Another perspective on the wake for each of the five velocity ratios is obtained from the nearly cross sectional views of figure 3.15. For each $V R$, smoke streaklines originating in three different constant $Z$ planes are shown. In most cases, wake structure is evident, and again, as was the case for the photographs in side view, the cross sectional views show a $V R$ dependence. For $V R=2$ and 4 , the widths of the wake and its vortices are similar for the two off-wall smoke-wire positions. On the other hand, for $V R=8 \& 10$, the wake and structure widths for $Z_{s w} / D_{j}=2$ are thinner than for $Z_{s w} / D_{j}=.5$. These observations coincide well with the side view photographs and comments associated with them.

Using a mirror angled at $45^{\circ}$, a simultaneous cross sectional and side view of the wake vortices is seen in figure 3.16 , where the smoke begins in the crossflow boundary layer. The correspondence between the two views is as would be expected.

All of the visual features of the wake structure discussed thus far are Reynolds number independent. One Reynolds number affect is seen in figure 3.17, where 
wakes at the crossflow wall for $R e_{c f}=3800,7600 \& 11400$ are compared. The structure at larger scales generally does not differ among these three cases, but the finer, smaller-scale turbulence, however, is more evident as $R e_{c f}$ increases. 


\section{Chapter 4}

\section{Sources of vorticity}

As is seen in chapter 3 , the near field of the transverse jet is dominated by several kinds of coherent vortical structures. It is therefore of interest to understand their origin and formation, i.e., to understand the source of the vorticity from which they are comprised. The principle motivation, however, for discussing sources of vorticity in this chapter is that the primary result of this research, which concerns the origin of the wake vorticity, relies heavily on a clear understanding of vorticity generation. While the discussion here is somewhat general, chapter 5 specifically addresses the source(s) of vorticity for the wake of the transverse jet.

To our knowledge, in every paper in which the wake formation of transverse jets has been discussed or mentioned previously, the wake vorticity has been described as vorticity "shed" from the jet and/or as vorticity due to a jet/crossflow interaction analogous to a solid cylinder/crossflow interaction. This has been the case even though it is apparently well known that in flows of uniform density, for instance, vorticity can be introduced into the flow only at solid surfaces. Since the jet does not offer the crossflow a solid surface, however, such analogies to solid cylinder wakes would appear to be irrelevant and incorrect.

In this chapter, the appropriate vorticity transport equation for the present flow is introduced, and the precise ways in which vorticity can be generated are presented. 


\subsection{The vorticity transport equation}

The most general vorticity equation is given by

$$
\frac{\rho D(\vec{\omega} / \rho)}{D t}=\vec{\omega} \cdot \nabla \vec{u}+\frac{1}{\rho} \nabla \times \nabla \cdot \underline{\underline{R}}-(\nabla \cdot \underline{\underline{R}}-\nabla p) \times \nabla\left(\frac{1}{\rho}\right)+\nabla \times \vec{f}
$$

where $\vec{\omega}$ is the vorticity, $\underline{\underline{R}}$ is the frictional stress tensor, and $\vec{f}$ is a body force per unit mass (Hornung 1988). Under the assumptions valid for the present flow, namely Newtonian, barotropic, isothermal, and incompressible flow with no body forces, this equation reduces to the more familiar vorticity transport equation

$$
\frac{D \vec{\omega}}{D t}=\vec{\omega} \cdot \nabla \vec{u}+\nu \nabla^{2} \vec{\omega}
$$

As emphasized by Morton (1984), this equation does not explicitly contain any vorticity source or generation terms; it shows only that vorticity is convected, stretched, turned, and diffused. The second term, the vortex stretching and turning term, is nonzero in three dimensional flows only and is necessarily zero at all solid boundaries. The third term allows for the cross-diffusion of vorticity of opposite sign and for the transport of vorticity transversely to streamlines.

Since equation 4.2 does not have a source term, new vorticity can only enter a flow through imposed initial conditions and/or wall boundary conditions; there are no sources of new vorticity within the flow. This is a fundamental point when considering the formation of vortical structures and, in particular, the formation of the wake vortices of transverse jets.

A distinction between new vorticity and vorticity which results from internal processing of vorticity already in the flow is implied. For instance, the second term in equation 4.2 can produce a new component of vorticity by turning preexisting vorticity. This is not new vorticity; it is instead the processing of vorticity which already is present. Perhaps a better interpretation of new vorticity is to consider 
it affecting a local change in the flow circulation. Continuing with the second term of equation 4.2 , the turning of vorticity conserves circulation around a closed fluid contour (neglecting diffusion), as Kelvin's theorem states. New vorticity, as it is considered here, is synonymous with adding circulation to or subtracting circulation from the flow.

\subsection{Vorticity generation}

As is shown in section 4.1, new vorticity enters a Newtonian, barotropic, isothermal, and incompressible flow through imposed initial conditions and/or wall boundary conditions only. Consider, therefore, vorticity generation at solid walls.

Defining the vorticity flux out of a wall as $\hat{n} \cdot \underline{\underline{J}}_{0}$, where $\underline{\underline{J}}_{0}=-\nu(\nabla \vec{\omega})_{0}$ is the vorticity flux tensor at the wall, and $\hat{n}$ is the wall-normal unit vector, it can be shown (Wu, Wu \& Wu 1987) that for nonaccelerating and nonrotating surfaces

$$
\rho \hat{n} \cdot \underline{J}_{0}=-\hat{n} \times(\nabla p)_{0}-\hat{n}\left(\hat{n} \cdot\left(\nabla \times \vec{\tau}_{0}\right)\right)+\left(\hat{n} \times \vec{\tau}_{0}\right) \cdot \nabla \hat{n}
$$

Incompressible flow and the absence of viscosity gradients are assumed in the derivation of this equation. The quantity $\hat{n} \cdot \underline{J}_{0}$ has the units of vorticity times a velocity and has a variety of labels in the literature; Lighthill (1963) refers to it as a vorticity source strength, and $\mathrm{Wu}, \mathrm{Wu} \& \mathrm{Wu}$ call it the "boundary kinematic vorticity flux." Essentially, it represents the rate of inflow of vorticity per unit wall area (Hornung 1988), here referred to as the vorticity flux out of a wall.

The first term on the right side of equation 4.3 is the vorticity source term due to a wall pressure gradient. The role of this term is well known, and incorrectly it is often considered to be the sole vorticity source at solid boundaries. It is only in two dimensional planar flow, for which the second and third terms in equation 4.3 are identically zero, that the pressure gradient term is the only vorticity source 
term at the wall. Therefore, vorticity generation analysis in two dimensions cannot be generalized to three dimensions; the contributions from the second and third terms of equation 4.3 are lost.

The generation of vorticity by the pressure gradient term is important for boundary layer separation. It is well known that an adverse pressure gradient generates vorticity at the wall of opposite sign to that of the initial boundary layer vorticity; in two dimensional and steady flow, once the vorticity at the wall reaches zero, the boundary layer separates. Furthermore, the favorable pressure gradient on the upwind side of a circular cylinder generates vorticity for its wake. The shedding process ultimately transports the vorticity generated at the wall into the wake.

As vorticity tangent to a surface is produced at the wall, it diffuses away from the surface to enter the flow. Wu, Wu \& Wu call this the "ascending mechanism" of introducing vorticity, and, furthermore, they state that for three dimensional, attached, and steady flow this pressure gradient term is the main contribution to the vorticity flux out of a wall.

In three dimensional flows, the second and third terms of equation 4.3 are, in general, nonzero. The second term accounts for the gradient of wall-normal vorticity due to a wall shear stress $\vec{\tau}_{0}$ with a nonzero $\nabla \times \vec{\tau}_{0}$ wall-normal component. Since the vorticity at a wall must be tangential to the surface, the wall-normal component of vorticity is zero at a wall. Immediately above the wall, however, a wall-normal component of vorticity can exist.

With spiral flows oriented normally to and above a wall, $\hat{n} \cdot\left(\nabla \times \vec{\tau}_{0}\right)$ is nonzero, and subsequently this term may be significant. In fact, the analyses of $\mathrm{Wu}, \mathrm{Gu} \&$ $\mathrm{Wu}(1987)$ and $\mathrm{Wu}, \mathrm{Wu} \& \mathrm{Wu}$ show that the normal vorticity production term can be significant near separation lines. They conclude that this process is responsible 
for the large wall-normal vorticity associated with a "horn vortex" or a "tornadolike vortex". Recall that the vorticity at the wall must still be parallel to the wall. Therefore, Wu, Wu \& Wu label this phenomenon as a "turning-up mechanism" since it involves the turning-up of vortex lines originally at the solid boundary.

The third term on the right hand side of equation 4.3 accounts for the effects of wall curvature with a component of curvature transverse to the wall shear stress direction. As with the pressure gradient term, this term produces vorticity tangent to surfaces. The vorticity diffuses away from the wall to enter the flow. To see this physically, consider axial flow along the surface of a circular cylinder. Also consider an imaginary circular vortex filament which diffuses from the surface. As it diffuses outwards in the radial direction, the filament must stretch. As this imaginary vortex filament stretches, its vorticity must increase, even though the net circulation of the boundary layer remains constant. This vorticity increase due to the necessary stretching during the ascension and expansion of the vortex filament is accounted for by this term; of course, with no transverse curvature, the filament would not stretch during ascension and this term would be zero. In a sense, this is a correction term to account for the surface curvature. Similar arguments can also be made for flow within circular pipes.

The vorticity flux out of a wall can also be expressed as

$$
\rho \hat{n} \cdot \underline{J}_{0}=-\nabla \times \vec{\tau}_{0}
$$

This shows that a rotational wall shear stress will lead to the generation of new vorticity. The advantage of using the form of equation 4.3 is that each of its three terms represents a separate physical cause for the generation of new vorticity at solid boundaries. 


\subsection{Implications}

For the incompressible and barotropic flow of concern in this research, the sources of fresh vorticity are at solid boundaries or walls. Flows do exist, however, where new vorticity is generated within the fluid. For example, in flows such as compressible boundary layers, stratified flows, and flows through curved shocks, vorticity is generated away from walls. An additional baroclinic torque term in the vorticity transport equation is responsible.

It has been shown that for flows which are Newtonian, barotropic, isothermal, incompressible, and free of any body forces, the sources of vorticity for the flow must be at solid boundaries (assuming that the initial conditions are such that the initial vorticity is zero). In the present flow there are, therefore, two possible sources of vorticity for the vortical structures which have been observed:

namely, the boundary layer within the jet nozzle and the boundary layer on the crossflow wall.

Statements which claim that the wakes of transverse jets form in the same or even similar manner as the wakes shed from solid circular cylinders or other solid objects are physically incorrect. The vorticity in the wakes of solid objects is vorticity which was generated at the surface of the object and then shed into the wake. As is shown in chapter 5, a new, entirely different mechanism is responsible for the wake formation of the transverse jet. 


\section{Chapter 5}

\section{Wake of the transverse jet}

Photographs in chapter 3 show that the wake of the transverse jet contains coherent vortical structures. Only a few investigators have measured or visualized structure in the wake region previously. McMahon et al. (1971) and Moussa et al. (1977) found characteristic wake frequencies from their hot-wire measurements, while McAllister (1968) and Reilly (1968) extracted wake Strouhal frequencies from flow visualization. McMahon et al. also visualized structure in the wake of the jet by placing a mesh of tufts in the wake, oriented parallel to the crossflow wall. Kuzo \& Roshko (1984), using dye injected within the wake, observed that wake structures persist several hundred jet diameters downstream of the orifice. Their visualizations showed the wake vortices most clearly. More recently, Wu, Vakili \& Yu (1988) visualized coherent wake vortices behind asymmetric jets in crossflow, referring to them as "spin-off" vortices. These vortices were visualized by placing the dye port in the wake just downstream of the jet. They did not, however, observe wake (or "spin-off") vortices for symmetric jets. Neither analytical nor computational models of transverse jets have incorporated the wake vortices.

To date, the accepted point of view has been that the wake vortices of transverse jets are analogous to solid cylinder wake vortices. In most studies, the counterrotating vortex pair of the jet is the focus, and any mention of the wake is 
usually in the form of an appeal to one or more of the references listed above.

Section 5.1 specifically addresses the source of vorticity for the wake and the wake formation mechanism. Therefore, results specific to the wake of the transverse jet are included. Experimental results such as wake-specific flow visualization, wake Strouhal frequencies, and wake velocity deficit data are presented and are used to determine the source of vorticity for the wake. The results show that the crossflow boundary layer is the source of the wake vorticity, and analysis suggests that the crossflow boundary layer can be expected to separate near the aft side of the jet, initiating the formation of the wake structures.

Section 5.2 presents additional, more general, wake results and characteristics. These include detailed wake Strouhal measurements to delineate the dependence of the wake on various experimental parameters: specifically, $V R, R e_{j}$, $L_{j} / D_{j}$ and $\delta_{c f} / D_{j}$. Total pressures along the wake centerline are also shown.

\subsection{Source of wake vorticity and wake formation model}

As mentioned in chapter 4 and earlier in this chapter, the formation of the wake vortices is usually attributed to shedding as vorticity sheds from circular cylinders or other solid objects. This implies that the source of vorticity for the wake structures is vorticity supposedly generated at the interface between the jet and the crossflow, just as vorticity in the wake of a cylinder is generated at the solid surface of the cylinder and then shed. Even though the wake of the transverse jet shares some similarities with the wake of a circular cylinder, flow visualization shows no analogous separation of the crossflow fluid as it passes over the jet body. Compare, for example, figure $5.1 \mathrm{a}$ with $5.1 \mathrm{~b}$. There is a striking difference between the wakes, 
particularly just downstream ${ }^{1}$ of the jet and cylinder. The separating streaklines are clearly seen coming off the top and bottom of the cylinder, a situation not present in the case of the jet. In both of these cases, $Z_{s w} / D_{j}=1$ (or, equivalently, $\left.Z_{s w} / D_{c}=1\right)$ and $R e_{c f}=7600$.

The formation of the wake of the transverse jet must be due to a mechanism very different from a cylinder shedding vorticity. For a solid cylinder, the vorticity generated at its surface is due to the pressure gradient vorticity flux term in equation 4.3. The favorable pressure gradient on the upstream surface of the cylinder generates vorticity of the appropriate sign for the wake vortices. There is no such mechanism in the present case of a flow around a jet, since there is no no-slip condition at the jet/crossflow interface. As discussed in chapter 4, vorticity generation theory restricts the source of vorticity to solid boundaries. The vorticity in the wake of the jet must be vorticity which originated either within the jet nozzle or at the crossflow wall.

Which one, then, is the source, and how do the wake structures form? These questions are addressed presently.

\subsubsection{Smoke as a vorticity marker}

The first step in determining the source of the wake vorticity is to use smoke to track vorticity carried by the boundary layer from within the nozzle and by the boundary layer on the crossflow wall. Smoke is considered to be a suitable marker of vorticity for the present purpose. Due to the relatively large particle mass of smoke (compared to the mass of air molecules), the diffusivity of smoke is much lower than the molecular diffusivity of air. Cimbala (1984) estimates the "effective Schmidt number" of smoke (ratio of smoke diffusivity to molecular

\footnotetext{
${ }^{1}$ Upstream and downstream are used in relation to the crossflow direction.
} 
diffusivity) to be of order $10^{5}$. Since the Schmidt number represents the ratio of viscous to molecular diffusivity and is of order unity for air, the ratio of the smoke diffusivity to viscous diffusivity for air is of order $10^{5}$. The important point is that vorticity diffuses much faster than smoke does; it is assumed that once the vorticity is tagged, the smoke marks the cores of vortices. Diffusion acts to spread the vorticity away from the cores faster than the smoke.

The suitability of using smoke as a vorticity marker is helped by the high Reynolds numbers here. The diffusion time scales for both smoke and air are long compared to the convective time scales. Within the time associated with the convection of the structures through the photographs' fields of view, diffusion of both smoke and vorticity is visually insignificant.

Figures 5.2a \& $\mathrm{b}$ show the same view of the flow field at $V R=4$ and $R e_{c f}=3800$. In both photographs, the jet issues from the bottom, left side of the photograph. Compare case (a), where the jet fluid (part of which is the boundary layer within the nozzle) is tagged by seeding the jet supply with cigarette smoke, with case (b), where the crossflow boundary layer is tagged with smoke ${ }^{2}$. In figure 5.2a, a well-defined and deflected jet is seen with no presence of smoke nor, apparently, jet fluid in the wake. Conversely, in figure $5.2 \mathrm{~b}$, most of the smoke ends up in the wake vortices. In fact, analogous comparisons can be made for the whole range of $V R$ from 2 through 10; compare, for example, figures 3.4a-e with figures 3.11a-e, respectively.

These results suggest that nozzle or jet vorticity does not contribute to wake vorticity. Not only does the jet not act like a solid cylinder, not generating any

\footnotetext{
${ }^{2}$ The crossflow boundary layer is tagged by placing the smoke-wire parallel to and just upstream of the leading edge of the crossflow wall, just to the positive $Z$ side of the stagnation streamline hitting the leading edge of the wall. This is indicated as $Z_{s w} / D_{j}=0+$.
} 
new vorticity, it also does not appear to shed any of its vorticity to the wake. Since smoke originating in the crossflow boundary layer, and therefore marking its vorticity, leaves the wall and visualizes the wake vortices, indications are that the source of the wake vorticity is the crossflow boundary layer.

To see jet vorticity entering the wake region would not have violated the constraints of vorticity generation theory; "shedding" of the jet's own vorticity by some peeling off and turning process would be allowable. Nothing like this is seen here nor elsewhere in the literature. Keffer \& Baines (1963), Kamotani \& Greber (1974) and Chassaing et al. (1972) show photographs of transverse jets seeded with smoke. In each case, only a deflected jet is observed; no smoke is seen in the wake region. No reference was found which showed tagged jet fluid entering the wake, as the wake region is defined here. Furthermore, Kamotani \& Greber (1972) studied a heated transverse jet. Temperature contours showed that the excess heat downstream of the orifice was confined to the deflected jet and did not, apparently, contaminate the wake with heat.

\subsubsection{Observations of crossflow boundary layer "separation events"}

If the crossflow boundary layer vorticity is indeed the source for the vorticity in the wake vortices, what is the mechanism by which this vorticity leaves the wall to enter the wake region? In what way is the crossflow boundary layer separating in order to produce the wake vortices?

First, the crossflow boundary layer separates ahead of the jet and forms a system of horseshoe vortices. This is not surprising and is understood. These structures are seen in figure 3.8.

Upon closer inspection of the photographs showing the near wall flow, what appear to be separations of the crossflow boundary layer just downstream of the 
orifice are observed. Consider figure 5.3, where smoke initially tags the crossflow boundary layer $\left(Z_{s w} / D_{j}=0+\right)$. The perspective is such that the jet is issuing at the viewer. Upstream of the jet, a portion of the boundary layer separates and forms a horseshoe vortex. The boundary layer fluid beyond the horseshoe structure on the $+Y$ side of the jet flows around the jet and then separates on its lee side. The arrow in the photograph indicates a vortex which has rolled up after the boundary layer separated. Such a crossflow boundary layer separation is referred to as a "separation event." The roll-up from the previous separation event, on the $-Y$ side of the jet, has convected further downstream. In this case, and it appears to be true in many cases, the separation events alternate from one side of the jet to the other.

After separation, the vorticity from the crossflow boundary layer is "free" to convect, stretch, turn, and diffuse, as the vorticity transport equation (equation 4.2) shows. That portion of vorticity closest to the jet is entrained and convected by the jet, thus establishing a connection between the wake vortices and the jet. The vertical $(Z)$ extensions of the separation event roll-ups into the jet are the wake vortices which are observed. The rotation of wake vortices whose separation events are on the $+Y$ side of the jet is clockwise. The other end of each separation event remains attached to the wall, as it must; the vortex "sheet" of the boundary layer cannot be cut. This process of turning vorticity initially parallel to the wall is reminiscent of the "turning up mechanism" of $\mathrm{Wu}, \mathrm{Wu} \& \mathrm{Wu}(1987)$, as discussed in chapter 4. The "footprints" of the wake structures are seen in figure 5.3 as the denser, white patches near $Y=0$. Four are seen in this photograph. The various features discussed here are indicated on the sketch in figure 5.4.

Figure 5.5 shows a simultaneous cross sectional and side view of the wake, obtained with a mirror placed at $45^{\circ}$ in the wind tunnel. Here, again, the smoke 
begins in the crossflow boundary layer. This shows that the "footprints" of the vortices correspond well to their positions in the wake. The birth of a wake vortex is seen with the separation event roll-up on the $+Y$ side of the jet. The two arrows on the photograph indicate the separation event roll-up and point to the same location for each view.

The significance of the separation events did not became apparent until after viewing videos of smoke-wire flow visualization. Viewing the flow from the side showed periodic vortical-like roll-ups just downstream of the jet and near the crossflow wall. A connection between the separation event roll-ups and wake vortices was noticed as smoke followed a path away from the wall at the lee side of the jet and along a wake structure. The newly formed structures, extending from the crossflow wall to the jet, then were observed to convect downstream.

Even though figure 5.3 represents one of the clearer and more apparent cases of a separation event, such crossflow boundary layer separations are typically seen at all velocity ratios. The appearances of separation events, in regards to the coherence and location of their associated roll-ups, vary with $V R$. Figure 5.6 shows typical near wall, very near wake photographs at each of five velocity ratios. The arrow in each photograph indicates a roll-up from a crossflow boundary layer separation event. In some cases, single events are not clearly distinguishable, but that the crossflow boundary layer fluid leaves the wall and enters the wake is still clear. Consider again, for instance, figure 3.11. In the photographs of figure 3.11, which show the wake vortices in side view, smoke initially in the crossflow boundary layer visualizes wake structure at each velocity ratio. Figure 5.7 shows clearer visualizations of separation events for $V R=4,6,8 \& 10$. A qualitative $V R$ dependence of the near wall, very near wake region of the flow is evident from the nine photographs of figures 5.6 and 5.7. 


\subsubsection{Wake flow patterns}

In order to clarify the role and presence of the crossflow boundary layer separation events and their connections to the wake structures, a crossflow wall surface topology is shown in figure 5.8. Figure 5.8 shows a possible surface streamline pattern specifically for the flow shown in figure 5.3 (Hornung \& Perry 1984, Perry \& Hornung 1984, Hornung 1990). Separation lines and attachment lines associated with the separation events and a horseshoe vortex are shown. Critical points ( $\mathrm{N}=$ node, $\mathrm{S}=$ saddle point) are also indicated. This surface topology represents an instantaneous pattern which matches the photograph and is consistent with the structures observed. For instance, the separation line associated with the separation event closest to the jet is the one labelled $A B$ in the figure. The surface streamlines between this separation line and the associated attachment line $\mathrm{AC}$ to the near side of the saddle/node points indicate axial flow toward the jet, as is expected.

Note also the darker, smokeless region just downstream of each separation event roll-up in figure 5.3. These regions correspond to the regions just downstream of the attachment lines $A C, A^{\prime} C^{\prime}$ and $A^{\prime \prime} C^{\prime \prime}$ of figure 5.8. That no smoke is in these regions is consistent with the topology.

Figure 5.9 shows a view of a separating streamsurface associated with a separation event. Again, the drawing is based on the flow shown in figure 5.3. Here, the separation event closest to the jet is considered as a case study. The drawing indicates how vorticity originally parallel to the wall is turned to form a wake vortex. Although the precise connection between the wake vortices and the jet is not understood, it appears that the portion of the separated boundary layer vorticity closer to the jet is entrained by the jet and therefore pulled away from 
the wall, as is indicated in figure 5.9. Relevant to this are the results of Kiya et al. (1986). Kiya et al. studied the interaction of vortex pairs and rings with a plane shear layer. For the case of strong vortex rings injected into a plane shear layer at some relative angle, they found that the vortex rings entrained and carried with them the shear layer vortices which were in their path. The relevant analogy here is to consider the succession of (distorted) vortex rings from the nozzle as entraining and carrying portions of the separation event roll-ups with them.

Figures $3.11,5.2 \mathrm{~b} \& 5.5$ clearly show that the wake vortices extend from the crossflow wall into the jet. Figure 5.10 shows the near wake for $V R=5.8$. Here, two wake vortices are seen "attached" to consecutive structures on the trailing edge of the jet, as indicated by the two arrows. Although this is not clearly typical, it may add insight into the connection between the wake and jet. Also, due to the "crease" in the cross section of the jet at its lee side (see, for instance, figure 3.5 right), it appears as if the wake vortices attach to the jet in the crease, near $Y=0$. The entrainment pattern around the jet, indicated in figures 3.7c (center), d (center) \& e (center), supports this.

The separation events, in all likelihood, represent only the inception of the wake structures. Vorticity near the wall can be continuously fed into the wake structures as they convect downstream. This is generally what is observed. Videos show that even after the formation of a wake structure, some spanwise or axial flow along it from the crossflow wall to the jet is seen, at least within the near field. Additionally, photographs where $Z_{s w} / D_{j}=0+$ show that the width of the disturbed flow at the crossflow wall grows with downstream distance $X$. (See, for examples, figures 3.15 (tops), $3.17 \&$ 5.3.) This suggests a funnel effect, in the sense that crossflow boundary layer fluid is continually entrained into the wake structures. 


\subsubsection{Wake vortices and separation events - Comparing characteristic (Strouhal) frequencies}

So far, flow visualization suggests that the source of wake vorticity is the crossflow boundary layer. Smoke, tagging the crossflow boundary layer fluid visualizes the wake vortices and shows crossflow boundary layer separation events which appear to be the beginnings of wake formation.

In order to make a quantitative connection between the wake vortices and separation event roll-ups, the characteristic (Strouhal) frequencies associated with the two types of structure were measured. The wake vortices were found to convect with characteristic frequencies. Likewise, the separation events occurred at characteristic frequencies. It is of interest, then, to compare these frequencies.

Frequency measurements were made using hot-wire anemometry. A TSI 1210-T1.5 hot-wire was placed both in the wake of the jet and near the crossflow boundary layer separation events. Wake power spectral peak frequency $\left(f_{w}\right)$ measurements were made at $X / D_{j}=3.5, Y / D_{j}=1.5$, and $Z / D_{j}=.5$. Although $f_{w}$ is independent of position within the wake, the sharpness of the spectral peak $f_{w}$ does vary.

The ability to pick-up "good" power spectra, i.e., ones with well-defined peaks, for the separation events was also dependent on location. Photographs, such as those in figures 5.6 and 5.7, were used to locate separation events. The probe was then traversed near that location to obtain a value for the separation event power spectral peak frequency $\left(f_{\text {sep }}\right)$.

The results are presented in terms of Strouhal numbers, defined here as $f_{w} D_{j} / U_{c f}$ and $f_{s e p} D_{j} / U_{c f}$ for $S t_{w}$ and $S t_{s e p}$, respectively. The Strouhal numbers are based on the rms average of 32 individual power spectra, obtained with an 
HP 3582A spectrum analyzer. (Appendix B gives more information about the spectrum analyzer.)

Figure 5.11 directly compares $S t_{w}$ and $S t_{\text {sep }}$ as a function of $V R$ at three crossflow Reynolds numbers. The agreement between $S t_{w}$ and $S t_{\text {sep }}$ is very good for each $R e_{c f}$. These results support what is suggested by the flow visualization of previous sections, that the source of wake vorticity is the crossflow boundary layer. Since the characteristic frequencies of the crossflow boundary layer separation events match those of the wake structures, an intimate connection between the two is suggested.

\subsubsection{Wake velocity profile effects of thickening the crossflow boundary layer}

It is of interest to study the effects of thickening the crossflow boundary layer. If the crossflow boundary layer is the source of wake vorticity, then changes in the crossflow boundary layer should be detected in the wake. In particular, are the larger displacement and momentum thicknesses of a thicker boundary layer detected as larger velocity deficits in the wake? Ideally, such comparisons should be made while keeping $R e_{c f}, R e_{j}$, and $V R$ constant.

Here, profiles of $U$ were measured in the wake of the jet for the four combinations of two velocity ratios $(V R=4 \& 8)$ and two crossflow wall lengths $\left(L_{j} / D_{j}\right.$ $=5 \& 10)$. Doubling the distance from the jet to the leading edge of the crossflow wall increases the nominal crossflow boundary layer thickness by about $40 \%$ (see Table 2.2). The boundary layer is laminar for both $L_{j} / D_{j}$.

Figures 5.12 through 5.14 compare the transverse jet $U / U_{c f}$ wake profiles for $L_{j} / D_{j}=5$ with those for $L_{j} / D_{j}=10$ at $V R=4$. Figures 5.15 through 5.17, similarly, compare $U / U_{c f}$ wake profiles at $V R=8$. In addition, $U / U_{c f}$ wake 
profiles for a wall-mounted circular cylinder of $A R=6$ are included in figures 5.12 \& 5.13. In all cases, $R e_{c f}=3800$. A single-wire hot-wire was used to measure the velocity profiles from the centerline, $Y / D_{j}=0$, to $Y / D_{j}=3$, where the data asymptote to some freestream value in most cases. Profiles were taken at four locations downstream from the orifice; $X / D_{j}=1.5,3.5,5.5$, and 7.5 , and at three distances from the crossflow wall, at $Z / D_{j}=.5,2.5$, and 4.5. The exception is for profiles at $Z / D_{j}=4.5$, where $X / D_{j}=1.5$ is clearly within the jet and not within its wake; therefore profiles were not measured there.

The mean velocity $U$ was obtained via King's Law on a 10 second average of the hot-wire voltage. The maximum turbulence level for each profile was generally about $20 \%$ of the crossflow velocity. Such a turbulence level introduces some error to the measured mean velocities, but since the fluctuations are comparable for $L_{j} / D_{j}=5$ and 10 , using the profiles to compare the two cases should still be accurate. In addition, there is significant flow in the $Z$ direction (along the span of the vortices). It is assumed that this does not significantly affect the $U$ values, for the hot-wire is most sensitive to flow in the $X$ and $Y$ directions.

Figures 5.12 through 5.17 show that the $U$ deficits are, in nearly all cases, greater for the thicker crossflow boundary layer $\left(L_{j} / D_{j}=10\right)$. That changing the crossflow boundary layer while keeping $R e_{c f}, R e_{j}$, and $V R$ constant affects the wake in such a way supports the role of the crossflow boundary layer as the source of the wake vorticity. If the crossflow boundary layer is a source of fluid for the wake, it is reasonable to expect larger wake velocity deficits for thicker crossflow boundary layers.

The wake velocity deficits are quantified by estimating wake displacement and momentum thicknesses. The wake displacement thickness nondimensionalized 
by $D_{j}$ is approximated by

$$
\frac{\delta_{w}}{D_{j}}=\int_{0}^{3}\left(1-\frac{U}{U_{\left(\frac{Y}{D_{j}}=3\right)}}\right) d\left(\frac{Y}{D_{j}}\right),
$$

while the wake momentum thickness nondimensionalized by $D_{j}$ is approximately given by

$$
\frac{\theta_{w}}{D_{j}}=\int_{0}^{3} \frac{U}{U_{\left(\frac{Y}{D_{j}}=3\right)}}\left(1-\frac{U}{U_{\left(\frac{Y}{D_{j}}=3\right)}}\right) d\left(\frac{Y}{D_{j}}\right) .
$$

Calculating the displacement and momentum thicknesses in these manners does not produce exact values for them; these are only estimates. Since only the $X$ velocity component, $U$, is used, any transfer of mass and momentum in the $Y$ and $Z$ directions is missed. In particular, the transfer in the $Z$ direction should not be omitted for accurate values of $\delta_{w}$ and $\theta_{w}$. For the purposes of an estimate, and in particular for comparing the wakes for the thin and thick crossflow boundary layers, the approximations given by equations $5.1 \& 5.2$ are assumed to be useful. It is assumed that the contribution from mass and momentum transfer in the other two directions would be similar in the two cases and would therefore not affect the comparison.

The thickness estimates for the cylinder wakes should be more accurate, for $W$ is smaller. As expected, flow visualization shows that axial flows along the cylinder wake vortices are much less than for the wake vortices of the jet. For example, figure 5.18 shows a side view of the flow about a cylinder with smoke beginning in the crossflow boundary layer. Within the field of view, the smoke remains within about $1 D_{c}$ of the wall. Contrast this with the corresponding side views of the jet (figure 3.11).

The calculated values for $\delta_{w} / D_{j}$ and $\theta_{w} / D_{j}$ are compiled in tables 5.1a, b \& c. Some of the velocity profiles shown in figures 5.12 through 5.17 are not typical wake-like profiles. For example, most of the profiles at $X / D_{j}=1.5$ have relatively 
large velocity overshoots exceeding the velocity at $Y / D_{j}=3$. Consequently, they produce negative values for $\delta_{w}$ and $\theta_{w}$. These profiles, therefore, are not considered wake-like. In general, any profile which contains velocities significantly larger than the value at $Y / D_{j}=3$ and/or any profile with a negative momentum thickness is considered not wake-like. The truly wake-like profiles are indicated by asterisks in tables 5.1a, b \& c.

Table 5.2 compares the average values of $\delta_{w} / D_{j}$ and $\theta_{w} / D_{j}$ for the thin and thick crossflow boundary layers at $V R=4 \& 8$, and for the $A R=6$ cylinder; the wake-like values from tables $5.1 \mathrm{a}, \mathrm{b} \& \mathrm{c}$ are averaged over position for each combination of $V R$ and $L_{j} / D_{j}$ (or $\delta_{c f}$ ). Values of $\overline{\theta_{w}} / D_{j}$ are $14 \%$ and $26 \%$ greater for the thicker crossflow boundary layer at $V R=4 \& 8$, respectively. Also, the averaged wake displacement thicknesses are $23 \%$ and $37 \%$ larger for the thicker case at $V R=4 \& 8$, respectively.

If the wake vortices formed by some other mechanism involving only the jet/crossflow interface and jet vorticity, changes in the crossflow boundary layer would not be expected to have such prominent effects on the wake profiles. This is especially true since the crossflow boundary layer thickness is small compared to the jet diameter. Instead, these results support the conclusion that the crossflow boundary layer is the source of wake vorticity.

\subsubsection{Why the crossflow boundary separates/Wake formation model}

The evidence from flow visualization and frequency measurements suggests that the wake formation of the transverse jet begins with the separation events. Why does the crossflow boundary layer separate near the lee side of the jet? An adverse pressure gradient at the wall must be responsible. What, then, is the reason for such an adverse pressure gradient? 
Figures 5.19a, b \& c show nearly cross sectional views of the wakes for an $A R=6$ wall-mounted cylinder, for a $V R=4$ transverse jet, and for a $V R=$ 8 jet, respectively. In each case, $Z_{s w} / D_{j}=0+$ and $R e_{c f}=11400$. Similarly, the photographs in figure 5.20 show the wakes for the same flow situations, only now $Z_{s w} / D_{j}=.5$. In this case, the telling difference between the very near wake regions of cylinder wakes and transverse jet wakes is evident. For the flows around the jets, the very near wake streaklines are closed. Conversely, the flow around the cylinder separates from its surface, thereby opening its very near wake.

Figure 5.21 shows close-ups of the flows in the immediate vicinity of the cylinder and jet; $Z_{s w} / D_{c}=Z_{s w} / D_{j}=.5$ and $R e_{c f}=3800$. The flow around the jet in figure $5.21 \mathrm{~b}$ looks nearly like potential flow around a circular cylinder; the very near wake streaklines are, again, closed. Figure 5.21a shows, of course, the real flow around a circular cylinder. While the flow around the jet at approximately $.5 D_{j}$ above the crossflow wall appears nearly potential, the flow near the wall is very different, as is clear from figure $5.22 \mathrm{c}$.

Coehlo \& Hunt (1989), in developing their numerical model, independently come to a similar conclusion. They state that, to a first approximation, the flow around a strong jet ("large $V R$ ") is potential flow around a circular cylinder, but with suction. Likewise, LeGrives' (1978) comments that the flow pattern around the jet is like the potential flow about a circular cylinder with a sink at the downstream side of the jet.

The potential-like streakline pattern around the jet has important implications for the crossflow boundary layer flow; the adverse pressure gradient on the lee side of the jet is imposed on the crossflow wall. This adverse pressure gradient is conducive to separating the crossflow boundary layer. This is shown with a Thwaites method calculation of the boundary layer on the crossflow wall, as- 
suming that the potential-like pressure gradient is present. The Thwaites method (Thwaites 1949) is based on the boundary layer equations, i.e., two-dimensional, steady, and laminar flow is assumed. Figure 5.22 shows the results of such an analysis. The outerflow potential-like streaklines are shown as are the separation lines predicted by the calculation for both $L_{j} / D_{j}=5$ and 10. Symmetry about $Y=0$ is assumed. As expected, the separation lines are slightly different for the two crossflow wall lengths. For each case, the separation line upstream of the jet is the separation leading to the horseshoe vortices. The separation line to the side and just aft of the jet coincides well with the separation events observed. Compare, for instance, figure 5.22 with figure 5.21c. When comparing the two, note that only the position of the roll-up of vorticity after separation is clear in the photograph; the corresponding separation line would be somewhat upstream from the roll-up.

The purpose of this analysis is not to predict the precise location of the separation events. If for no other reason, it was seen that their locations vary with $V R$, and this analysis does not take any $V R$ dependence into account. Furthermore, two-dimensional, laminar, and steady flow is assumed; the boundary layer and outer flows here, in general, are three-dimensional and unsteady. Nonetheless, what the analysis does show is that, given the observed outer flow which appears nearly potential, a separation of the crossflow boundary layer (in addition to the horseshoe separation) is expected. The potential-like outer flow imposes its pressure gradient on the wall. For the flow around a cylinder, which has an open very near wake, the crossflow boundary layer does not encounter an analogous adverse pressure gradient on the lee side; thus, a similar boundary layer separation does not occur. (The separation for the horseshoe vortices does still occur.) The separation from the surface of the cylinder itself relieves any possibility of an adverse 
pressure gradient on the crossflow wall which is analogous to that present for the jet in a crossflow.

\subsubsection{The principal message}

This section (5.1) on the wake formation of the transverse jet shows that the source of vorticity for the wake structures is the crossflow boundary layer. The system of vortices in the wake of a transverse jet is distinctly different in origin and formation from the vortices which are shed from a solid cylinder. In the case of the transverse jet, where the jet/crossflow interface cannot generate new vorticity, the wake vorticity comes from the boundary layer on the wall from which the jet issues. The crossflow boundary layer separates near the downstream side of the jet because it cannot negotiate the adverse pressure gradient which is imposed on it by the flow around the jet. The boundary layer fluid is then incorporated into the wake vortices, which extend from the wall to the deflected jet.

\subsection{Additional wake measurements}

Whereas the wake measurements and analysis of the previous section were used expressly to determine the source of vorticity for the wake structures, the experimental results which follow in this chapter serve to characterize the wake more generally.

\subsubsection{Characteristic wake (Strouhal) frequencies}

In section 5.1.3, frequencies measured in the wake were compared with those measured near the crossflow boundary layer separation events. The characteristic frequencies were found to match, supporting the premise that there is a connec- 
tion between the wake structures and separation events. Additional information can be educed from the $S t_{w}$ and $S t_{\text {sep }}$ data to characterize the wake more fully. This is the topic of this section.

\subsubsection{Some conventions for $S t_{w}$}

As before, all Strouhal numbers are based on the rms average of 32 individual power spectra, obtained with an HP $3582 \mathrm{~A}$ spectrum analyzer. Unless otherwise noted, the $S t_{w}$ data were measured at $X / D_{j}=3.5, Y / D_{j}=1.5$, and $Z / D_{j}=.5$. Although the spectra with the sharpest peaks were generally obtained near this location in the wake, the characteristic frequencies of the wake structures were not a function of position.

Each power spectrum or, more precisely, each rms average of 32 power spectra recorded was assigned a relative sharpness level, with a "4" indicating the sharpest spectral peak and "0" indicating the lack of a peak. The sharpness levels are admittedly arbitrary and only qualitative in nature. In general, only one peak associated with the wake structures is seen per spectrum; no subharmonics nor higher harmonics were seen. Examples of spectral sharpnesses 0 through 4 are shown in figure 5.23. For each spectrum, the abscissa includes $0 \leq f_{w} \leq 50$ $\mathrm{Hz}$. and the ordinate shows $\log (P)$. A relatively accurate peak frequency can be deduced for spectra of sharpnesses 2,3 , and 4 . In most cases, a peak frequency is also established for a spectral sharpness of 1 . In some cases, frequency ranges are applied to sharpness 0 spectra. Figure $5.23 \mathrm{a}$ indicates how a frequency range is inferred. Poor spectra with flat "peaks" or multiple peaks are indicated in $S t_{w}$ plots by vertical lines connecting the frequency extremes of the flat or multiple peak range.

The repeatability of the spectral measurements was quite good. For example, 
figure 5.24 compares results for four different runs at $R e_{c f}=3800$ and $L_{j} / D_{j}=$ 5 ; these runs spanned eight months. For clarity sake, figure 5.24 only includes spectral peaks of sharpness 1 through 4 . Repeated disassembly and reassembly of the experimental set-up occurred among the runs; the results appear to be quite robust in this sense.

\subsubsection{Parameter dependency}

The four relevant parameters which were varied in the experiments are $V R, R e_{c f}$ (or $R e_{j}$ if $V R$ is held constant), $L_{j} / D_{j}$, and $\delta_{c f} / D_{j}$. These parameters cannot, in general, be varied independently. For instance, $\delta_{c f} / D_{j}$ is affected by changes in either $R e_{c f}$ or $L_{j} / D_{j}$. The parameters $\delta_{c f} / D_{j}$ and $L_{j} / D_{j}$ take on new importance due to the finding that the crossflow boundary layer is essential for the wake vorticity. Here, the effects of varying these parameters on $S t_{w}$ are discussed.

Figure 5.25 is a compilation of five sets of $S t_{w}$ data. Combinations of the three crossflow Reynolds numbers and the two crossflow wall lengths comprise the five sets. Figure 5.25 also shows the average spectral sharpnesses of the $f_{w}$ peaks at different velocity ratios. The sharpness values shown are the averages of all the spectra in the five sets of data. Sharpness data for each $\Delta V R=.25$ are lumped together for averaging. These averages include spectra without discernable peaks, i.e., spectra with sharpness 0 . The $S t_{w}$ plot, on the other hand, only includes spectra with discernable peaks, i.e., spectra with sharpness values greater than or equal to 1.

Figure 5.25 shows that $S t_{w}$ is dependent on the jet to crossflow velocity ratio for each of the five sets of results. Also of importance is the dependence of the $f_{w}$ spectral peak sharpness on velocity ratio. Most striking are the very strong spectra recorded in the neighborhood of $V R=4$. Accompanying the strong spectra near 
$V R=4$ are $S t_{w}$ values nearly independent of $R e_{c f}$, as well as $\delta_{c f} / D_{j}$. Figure 5.26 shows $S t_{w}$ over a larger range of $R e_{c f}$ at $V R=4$. As indicated by figure $5.25, S t_{w}$ is independent of $R e_{c f}$ near $V R=4$. At $V R=4$, the average $S t_{w}$ over this range of $R e_{c f}$ is .13 .

The wake Strouhal numbers near $V R=2$ also appear relatively independent of $R e_{c f}$ and the other parameters. However, the average spectral sharpness is not as high as it is near $V R=4$. The average $S t_{w}$ at $V R=2$ is .16 .

The strength of the wake spectra reached a local minimum near $V R=3$. Coincident with this is broad scatter in the $S t_{w}$ results. Likewise, just below $V R$ $=6$, there is also a local minimum in the $f_{w}$ spectral sharpness graph and broad scatter in the $S t_{w}$ results. In fact, for $V R \geq 5.5$ there is significant dependence of $S t_{w}$ on the parameters, and the spectral sharpnesses are generally lower.

Some effects of $R e_{c f}$ can be inferred from figure 5.27. This figure shows spectral results for $R e_{c f}=3800,7600$, and 11400 with $L_{j} / D_{j}=5$ only. Here, the $f_{w}$ spectral sharpnesses are shown for each data point. (For poor spectra of sharpness 0 , peak ranges of $S t_{w}$ are indicated by the vertical lines in the $S t_{w}$ graphs.) Similarly, figure 5.28 shows the results with $L_{j} / D_{j}=10$. (The local minima in spectral sharpnesses are again visible near $V R=3$ and 6 , as is the local maximum near $V R=4$.) In general, there is no single trend with $R e_{c f}$. However, there does appear to be a monotonic trend of decreasing $S t_{w}$ with increasing $R e_{c f}$ near $V R=6.25\left(L_{j} / D_{j}=5\right)$. This is shown more clearly in figure 5.29. Moussa et al. (1977) state that $S t_{w}$ is nearly independent of $R e_{c f}$. The present results show that this is not the case for all velocity ratios.

Figure 5.30 shows the effects of changing $L_{j} / D_{j}$ on $S t_{w}$. These results do not conclusively show whether or not thickening the crossflow boundary layer at the same $V R$ and $R e_{c f}$ has significant effects on the wake frequencies. Certainly 
for $R e_{c f}=7600$, figure $5.30 \mathrm{~b}$ suggests that there is no effect. At $R e_{c f}=3800$, the agreement or lack of one is not as clear. The general dependence of $S t_{w}$ on $V R$ appears to remain unchanged however. In particular, $S t_{w}$ match near $V R=$ 2 and $V R=4$ for both crossflow lengths. Poor spectra exist near $V R=3$, begin again near $V R=5.5$, and persist to higher velocity ratios.

Recall that the Thwaites method calculations in section 5.1.5 show that the doubling of $L_{j} / D_{j}$ has a slight effect on the location of the calculated separation event. Increasing $L_{j} / D_{j}$ moved the separation lines upstream. It is not clear whether such a shift in the lines of separation was realized in the experiments, and if so, whether the wake Strouhal numbers were affected. Flow visualization of the separation events and wake structures with $L_{j} / D_{j}=10$ do not show any discernable differences from the case of the shorter crossflow wall.

Figure 5.31 displays the $S t_{w}$ results in terms of crossflow boundary layer thickness, $\delta_{c f} / D_{j}$. Four values of $\delta_{c f} / D_{j}$ are shown in figure 5.31 ; no trends are clear.

To summarize, $S t_{w}$ is found to be very dependent on the jet to crossflow velocity ratio. The most striking results are the characteristics for jets near $V R=$ 4. The strongest spectra are found near $V R=4$. Furthermore, $S t_{w}$ is independent of all parameters investigated near $V R=4$. Discontinuities, marked by wide scatter in values of $S t_{w}$ and poor spectra are found near $V R=3$ and 6 . In general, there are no clear trends with $R e_{c f}$. However, one is indicated near $V R=$ 6.25. Also, there are no clear $S t_{w}$ trends with $\delta_{c f} / D_{j}$, and the results of changing $L_{j} / D_{j}$ are not conclusive. 


\subsubsection{Comparison to others}

Wake Strouhal data measured by McAllister (1968), Reilly (1968), McMahon et al. (1971), and Moussa et al. are shown in figure 5.32. $S t_{w}$ values in figure 5.32 are in the same range as are the present results. $R e_{c f}$ varies among these results. No single crossflow Reynolds number is available over the whole range of $V R$. Therefore, direct comparison is difficult.

The best comparison possible is between the $R e_{c f}=7600$ and $L_{j} / D_{j}=5$ present data and the $R e_{c f}=8000$ and $L_{j} / D_{j}=2.7$ data of Moussa et al.. This comparison is shown in figure 5.33. The results agree quite well near $V R=4$. Moussa et al. do not, however, mention uniqueness of the wake or its spectra at or near $V R=4$. Recall that the present results indicate a much "stronger" wake near $V R=4$, in the sense that the wake structures convect with better periodicity. Possibly, there exists an analogous discontinuity near $V R=3$. In fact, Moussa et al. do comment that a change in "regime" occurs near $V R=$ 3. Although no elaboration is given, this may very well be similar to the poor spectra and discontinuity observed near a velocity ratio of three in the present results. Although not evident for their flush-mounted jet, a transition near $V R$ $=5.5$ is observed and commented on for their case of a jet whose supply pipe protrudes into the crossflow. Their results for this case are reproduced in figure 5.34. It is difficult to infer that this slight discontinuity for a protruding jet is analogous to that observed here near the same $V R$.

For the case of the protruding jet, the wake vortices of the jet are likely extensions of the vortices behind the circular cylinder protrusion. The shedding from the protruding pipe dominates the shedding frequency of the whole wake system. The values for $S t_{w}$ are closer to those expected behind a circular cylinder, i.e., 
$S t_{w} \approx .20$. Moussa et al. conclude the same based on the relative independence of $S t_{w}$ on $V R$ for a protruding jet.

\subsubsection{Cylinder $S t_{w}$ comparison}

It is well known that there are characteristic Strouhal numbers for the Karman vortex wake of flow past a circular cylinder. In general, the Strouhal number for a circular cylinder wake is dependent on $R e_{c f}$, but for the range of crossflow Reynolds numbers dealt with here, $S t_{w}$ for a cylinder lies approximately between .20 and .21 (Roshko 1953) The values for the wake of the transverse jet, therefore, are generally lower.

These cylinder $S t_{w}$ are for cylinders whose aspect ratios are large. The circular cylinder used here for comparison with the transverse jet has an aspect ratio of 6 ; one end is wall-mounted while the other is free. Frequency measurements in the wake of this cylinder and wall-mounted cylinders of different aspect ratios are shown in figure 5.35. As is quite apparent, $S t_{w}$ is very dependent on the cylinder aspect ratio. Although these data were taken close to the crossflow wall, the wake frequencies were essentially independent of position along the span of the cylinder. The present results agree well with those of Okamoto \& Yagita (1973), which are included in figure 5.35. Three-dimensional (or end) effects therefore become very significant at lower aspect ratios.

\subsubsection{More from the wake $\left(U / U_{c f}\right)$ profiles}

Estimates for the wake momentum and displacement thicknesses (from equations $5.1 \& 5.2)$ are shown in tables $5.1 \mathrm{a} \&$ b. In section 5.1.4, it is shown that the thicknesses are generally larger for the thicker crossflow boundary layer. Figures 5.36 and 5.37 show the dependence of $\overline{\delta_{w}} / D_{j}$ and $\overline{\theta_{w}} / D_{j}$, respectively, on distance 
from the crossflow wall. Each data point in these figures represents an average over $X / D_{j}$ of wake-like velocity profiles, i.e., those indicated by asterisks in tables 5.1a \& b. (Without averaging, the trend at each $X / D_{j}$ is similar to those shown in these figures.) The results show that the thicknesses at $V R=4$ increase with distance from the wall. However, at $V R=8$, the wake thicknesses remain roughly constant in $Z / D_{j}$. Other differences between wakes at $V R=4$ and 8 have been noted previously. Consider the differences in flow visualizations and $S t_{w}$ results, for instance.

These resuits aiso indicate that the measurements were truly made within the wake. At some larger $Z / D_{j}$, the jet would eventually be reached. At that point, momentum and mass excesses would be detected and the thicknesses would become negative.

\subsubsection{Wake total pressure measurements along $Y / D_{j}=0$}

Wake total pressure measurements on the $Y=0$ plane were taken using a Venturi Kiel probe. The total pressures measured with the probe depended on the direction in which it was pointed. The Kiel probe was rotated in the $Y=0$ plane until the maximum wake total pressure was measured. The results at $R e_{c f}=3800$ are shown in figures $5.38 \mathrm{a}, \mathrm{b} \& \mathrm{c}$ for $V R=4$ and 8 jets, and for the wall-mounted circular cylinder, respectively. The total pressure coefficient $C_{p t o t}$, defined as

$$
C_{p t o t} \equiv \frac{p_{t w a k e}-p_{t c f}}{\frac{1}{2} \rho U_{c f}^{2}}
$$

is plotted against the downstream distance from the nozzle or cylinder, as the case may be. Since all the data are negative, each result represents a total pressure deficit.

A total pressure deficit can indicate that there is dissipation in the flow, such 
as dissipation and the loss of fluid momentum due to the drag on the cylinder. In the case of the wake of a transverse jet, the total pressure deficit is more likely due to the transport of lower total pressure fluid from the crossflow boundary layer into the wake. Whether dissipation occurs solely due to the presence of the jet and accompanying mixing is not clear.

Close to the crossflow wall, the total pressure deficits for the cylinder wake is larger than for the jets' wakes. This result confers with the velocity data. Further away from the wall, closer to the end of the $A R=6$ cylinder, the total pressure deficit behind the cylinder is less than it is behind the jets. Due to the shortness of the cylinder, three-dimensional flow wrapping around the end of the cylinder likely reduces the wake momentum and total pressure deficits near its end.

A difference between the wakes at $V R=4$ and $V R=8$ is clear again by comparing figures $5.38 \mathrm{a} \& \mathrm{~b}$. For the lower velocity ratio, the total pressure deficit actually increases with distance from the crossflow wall, just as the displacement and momentum thicknesses did in figure 5.36a and 5.37a. Conversely, for the larger velocity ratio, the deficit generally decreases and then levels off at some distance from the wall. Here, likewise, the correspondence with figures $5.36 \mathrm{~b}$ and $5.37 \mathrm{~b}$ is good.

The wake total pressure results for the other two crossflow Reynolds numbers (7600 and 11400) show similar trends, as is seen in figures 5.39 and 5.40 . 


\section{Chapter 6}

\section{Near field development of the counterrotating vortex pair (CVP)}

Although the counterrotating vortex pair (CVP) is a dominant structure in the far field of the transvere jet, its study and understanding in the near field is also of interest. In particular, how is the CVP far field structure connected to its origin, and, continuing with the point of view established in chapter 5 for the wake structure, what is the source of vorticity for the counterrotating vortex pair? The discussion in chapter 4 shows that there is no generation of "new" vorticity at the jet/crossflow interface; the source of vorticity for the CVP vortices must be either the crossflow boundary layer or the boundary layer within the jet nozzle, or possibly both.

In any case, the source of vorticity for these structures is near the jet orifice, and therefore the vorticity transition from its source to CVP structure is likely in the near field. In this chapter, the vorticity source and development of the counterrotating vortex pair is addressed. Past analyses (Durando 1971; Broadwell \& Breidenthal 1984; Karagozian 1986) have used the impulse imparted by the jet on the crossflow to study the vortex pair. Here, the strength of the vortex pair is estimated by considering only the flux of vorticity from the jet nozzle. 


\subsection{CVP - A near field structure}

Figure 6.1 shows a sketch of the portion of the transverse jet relevant to this chapter along with notation used. There is evidence for the near field presence of a counterrotating vortex pair (CVP). Consider figures $6.2 \& 6.3$, which show a compilation of experimental and analytical results, respectively, from six references. Nondimensional circulation $\gamma$, defined as $\Gamma /\left(2 U_{c f} D_{j}\right)$, is plotted against the distance $s_{v}$ nondimensionalized by $D_{j}$. The symbol $\Gamma$ represents the circulation of one CVP vortex; by symmetry, the circulations of the two vortices in the pair are equal and opposite. The distance from the origin along the trajectory of a CVP vortex is given by $s_{v}$. Although there is disagreement among the results, one point is clear; the circulation is significant within several diameters of the orifice. In fact, the maximum in $\gamma$ is attained close to the orifice in most cases. For $V R=$ 8 , for example, the maximum in $\gamma$ occurs in the range $6 \leq s_{v} / D_{j} \leq 18$, depending on the reference. The maximum in $\gamma$ is reached earlier at lower velocity ratios. At $V R=4$, for instance, both experimental and analytical results show that the maximum circulation for a CVP vortex is reached by $s_{v} / D_{j}=4$.

The results in figures $6.2 \& 6.3$ suggest that the development of the counterrotating vortex pair is essentially complete in the near field, complete in the sense that $\gamma$ subsequently falls off going into the far field. The drop in $\gamma$ at larger $s_{v} / D_{j}$ is likely due to cancellation by cross diffusion of the two bound vortices, which are of opposite sign.

Moussa et al. (1977) measure a counterrotating vortex pair in the near field; see their figure 6 , which shows a CVP-like rotational velocity field at the planes $X / D_{j}=.5$ and 1.0. They project the velocity $\sqrt{V^{2}+W^{2}}$ on these planes for $V R$ $=3.5$. In addition, they use an integral form of the Reynolds-averaged vorticity 
transport equation to obtain an expression for the net flux of mean vorticity (see their equation 7). The convection of mean vorticity through the surfaces of a control volume is then balanced by four terms: the stretching and turning of mean vorticity term, the diffusion of mean vorticity term, a "turbulent vorticity" convection term, and a "turbulent vorticity" stretching and turning term. Within a control volume encompassing the jet from $X / D_{j}=-.5$ to $X / D_{j}=.5$, they show that fluxes due to the stretching and turning of mean vorticity and "turbulent vorticity" are very significant. The implication is that vorticity from the jet nozzle is deforming very near the orifice. Moussa et al. propose, therefore, that "the bound vortices are extensions of the vorticity rings emanating from the [nozzle]." Sykes et al. (1986), based on their numerical results, have also suggested that the ring-like vorticity of the jet may evolve into the counterrotating pair. In addition, the numerical results of Coehlo \& Hunt (1989) suggest that the evolution of jet vorticity into the CVP vortices may begin within the nozzle boundary layer; the effects of the crossflow are felt within the nozzle.

Flow visualization in chapter 3 shows near field structure which suggests a pair of counterrotating vortices. Refer to section 3.1.2 and the photographs of figures $3.5,3.6$ and $3.7 \mathrm{c}$ (center), d (center) \& e (center).

\subsection{The approach - Conservation of jet vorticity flux}

The hypothesis is that the source of CVP vorticity is the boundary layer vorticity issuing from the jet nozzle, i.e., the vortex pair evolves from the shear layer vorticity of the jet. (It appears that the crossflow boundary layer, which supplies the wake vorticity, is not likely to be a significant source of CVP vorticity.) Applying such a hypothesis, the flux of vorticity emanating from the nozzle (either $\Phi_{j}$ or 
$\left.\Phi_{j, x}\right)^{1}$ is matched with the flux of vorticity at the point where a CVP vortex is fully-developed $\left(\Phi_{1}\right) .{ }^{2}$ Full-development means that the CVP vortex has reached its maximum circulation. By matching the fluxes, an estimate for the maximum circulation of a CVP vortex is obtained and compared with published results. This is used as a test for the hypothesis.

\subsection{Vorticity flux analysis}

Since the vorticity, circulation, and vorticity flux in one CVP vortex is equal and opposite to that in the other, only one vortex of the pair is considered in this analysis. The subscript "1" indicates values associated with one vortex of the pair at the point of full CVP development. The maximum nondimensional circulation of one vortex of the counterrotating pair is given by the following expression:

$$
\gamma_{1} \equiv \frac{\Gamma_{1}}{2 U_{c f} D_{j}}
$$

where $\Gamma_{1}$ is the maximum circulation in one vortex of the pair, i.e., it is the circulation of a CVP vortex at its point of full-development. As is seen in figures 6.2 and 6.3 , there is a point along the vortex trajectory at which $\gamma$ is maximum. The distance to this point is $s_{v 1}$. Beyond $s_{v 1}$, the strength of each vortex of the pair characteristically decreases.

Consider that

$$
\Gamma_{1}=\bar{\omega}_{1} A_{1}
$$

where $\bar{\omega}_{1}$ is the magnitude of the mean vorticity of a CVP vortex, whose direction is oriented normal to $A_{1} ; A_{1}$ is the cross sectional area of one CVP vortex. One

\footnotetext{
${ }^{1}$ The distinction between $\Phi_{j}$ and $\Phi_{j, x}$ is made in section 6.3.1

${ }^{2}$ In general, the flux of vorticity is given here by $\left|\int_{S} \vec{\omega} \vec{U} \cdot d \vec{A}\right|$.
} 
can approximate $\bar{\omega}_{1}$ as

$$
\bar{w}_{1} \approx \frac{\Phi_{1}}{Q_{1}},
$$

where $\Phi_{1}$ is the vorticity flux in one CVP vortex at its full-development, and $Q_{1}$ is the volume flow rate of vortical fluid through $A_{1}$.

$$
Q_{1}=U_{1 \text { conv }} A_{1} .
$$

$U_{1 c o n v}$ is the mean convective velocity of vortical fluid through $A_{1}$ and is locally tangent to $s_{v 1}$.

Now, by substituting equations $6.3 \& 6.4$ into equation 6.2

$$
\Gamma_{1} \approx \frac{\Phi_{1}}{U_{1 \text { conv }}} .
$$

Neither $\Phi_{1}$ nor $U_{1 \text { conv }}$ is known without extensive measurements. Here, $\Phi_{1}$ is determined by applying the hypothesis of section 6.2, and $U_{1 \text { conv }}$ is estimated. A final estimate for the circulation is then obtained.

\subsection{1 $\Phi_{1}$ by hypothesis}

To directly calculate $\Phi_{1}$, the flux of vorticity in a CVP structure at $s_{v 1}$, one would need to know the details of the vorticity and velocity fields at $A_{1}$. By applying the hypothesis of section 6.2 , however, $\Phi_{1}$ is estimated from the conditions at the nozzle exit plane.

Two separate cases for the flux of vorticity from the nozzle are considered here. For case (a), the flux of all vorticity components from the nozzle is used. Doing so essentially results in an upper bound for $\Gamma_{1}$. For case (b), only the $X$ component of the nozzle boundary layer vorticity is included in the jet flux. The purpose of considering only the $X$ component is addressed in part (b) below. 
(a) The total nozzle vorticity flux (through one half of the orifice, considering symmetry) is given by

$$
\Phi_{j}=\left|\int_{A_{j} / 2} \vec{\omega} \vec{U} \cdot d \vec{A}\right|
$$

where $A_{j}$ is the nozzle exit area. Neglecting crossflow effects within the nozzle,

$$
\vec{\omega} \approx-\frac{\partial W}{\partial r} \hat{e}_{\theta}, \quad \vec{U} \approx W(r) \hat{e}_{z}, \quad d \vec{A}=r d r d \theta \hat{e}_{z} .
$$

(Refer to figure 6.4 for the notation used here.) It now follows from equation 6.6 that

$$
\Phi_{j} \approx \frac{1}{2}\left|\int_{0}^{\pi} \int_{0}^{U_{j}^{2}} r d W^{2} d \theta\right| .
$$

Table 2.3 shows that the boundary layer thickness at the nozzle exit is small compared to the jet $\left(.029 \leq \delta_{j} / D_{j} \leq .045\right)$. Therefore $r \approx D_{j} / 2$, i.e., the nozzle exit boundary layer is considered to be infinitesimally thin, or, equivalently, the exit velocity profile is assumed top hat-like. This is an appropriate approximation for the present experiments and for the references mentioned in this chapter. ${ }^{3}$ Finally, then, the total vorticity flux from the nozzle is estimated to be

$$
\Phi_{j} \approx \frac{\pi D_{j} U_{j}^{2}}{4}
$$

(b) In case (a), the total vorticity flux from the nozzle is estimated. As will be seen, this yields an upper limit to the CVP vortex circulation. Here, in case (b), it appears plausible to include only the $X$ component of the nozzle boundary layer vorticity in the flux equation 6.6 (hence the subscript " $x$ " in $\Phi_{j, x}$ ). The reasoning behind this is the following. One can create an idealized view that, as the jet develops, the leading and trailing edges of the jet essentially cancel $Y$ vorticity contributions. Consider figure 6.5. Figure $6.5 \mathrm{a}$ shows a side view skeletal sketch of the developing transverse jet. The rings are meant to model the distorted

\footnotetext{
${ }^{3}$ Thompson (1972), however, does not report the jet velocity profile.
} 
ring-like vortices which comprise the bending jet and are drawn equally spaced and horizontal for simplicity.

From the flow visualization photographs, the ring-like vortices are known not to be necessarily parallel nor to be uniformly spaced. Distortion (turning and stretching) of the initially nearly annular shear layer occurs. In some cases, portions of the rings are seen to significantly rotate (see, e.g., figure 3.2). With this in mind, this view is admittedly very idealized, but may still be indicative of the mean behavior of the developing jet.

Figure $6.5 \mathrm{~b}$ shows the projection of the series of ring vortices onto a plane. The rings convect with the jet. Notice that the rings' positive and negative $Y$ vorticity $(\eta)$ cancels due to the overlap of the upstream and downstream portions of the rings. For instance, if one were to make jet measurements at some $X$ location downstream of the orifice which average over time, the alternating positive and negative $Y$ vorticity would average to zero as the rings convect by. Conversely, the $+X$ vorticity of successive rings is additive, as are the $-X$ components. In addition, the closer proximity of the $+Y$ and $-Y$ components of vorticity provides for their quicker cross-diffusion and cancellation. The end result of either or both time-mean measurements and diffusion is a pair of counterrotating vortices, as is shown at the bottom of figure $6.5 \mathrm{~b}$.

Implicit to using the entire $X$ vorticity flux from the nozzle is that no cross diffusion of $X$ vorticity has occurred between the orifice and $s_{v 1}$, i.e., that no cancellation of $X$ vorticity has occurred.

For this estimate, the flux of jet $X$ vorticity is given by

$$
\Phi_{j, x}=\left|\int_{A_{j} / 2} \xi \vec{U} \cdot d \vec{A}\right|
$$

where $A_{j}$ is the nozzle exit area, and $\xi$ is the $X$ component of vorticity at the 
orifice. Again, neglecting crossflow effects within the nozzle,

$$
\xi \approx-\frac{\partial W}{\partial r} \sin \theta, \quad \vec{U} \approx W(r) \hat{e}_{z}, \quad d \vec{A}=r d r d \theta \hat{e}_{z} .
$$

Now, it follows that

$$
\Phi_{j, x} \approx \frac{1}{2}\left|\int_{0}^{\pi} \int_{0}^{U_{j}^{2}} r d W^{2} \sin \theta d \theta\right| .
$$

The flux of jet $X$ vorticity is estimated to be

$$
\Phi_{j, x} \approx \frac{D_{j} U_{j}^{2}}{2}
$$

By hypothesis, the flux of vorticity at the point of a CVP vortex fulldevelopment $\left(\Phi_{1}\right)$ is matched to each jet vorticity flux estimate, i.e., $\Phi_{1} \approx \Phi_{j}$ and $\Phi_{1} \approx \Phi_{j, x}$. Therefore

$$
\Phi_{1} \approx \frac{\pi D_{j} U_{j}^{2}}{4}, \quad \Phi_{1} \approx \frac{D_{j} U_{j}^{2}}{2} .
$$

(The left equation of each pair from here on corresponds to the use of $\Phi_{j}$, while the right equation corresponds to the case with $\Phi_{j, x}$ ). Recall that equation 6.14 a supposes that all of the vorticity from the nozzle comprises the vortex pair. On the other hand, equation $6.14 \mathrm{~b}$ supposes that only the $X$ component of nozzle vorticity contributes to the vortex pair.

Now, by substituting equations $6.14 \mathrm{a} \& \mathrm{~b}$ into equation 6.5 ,

$$
\Gamma_{1} \approx \frac{\pi D_{j} U_{j}^{2}}{4 U_{1 \text { conv }}}, \quad \Gamma_{1} \approx \frac{D_{j} U_{j}^{2}}{2 U_{1 \text { conv }}} .
$$

The remaining unknown in equations $6.15 \mathrm{a} \& \mathrm{~b}$ is the mean convective velocity $U_{1 \text { conv }}$, an estimate for which follows.

\subsubsection{An estimate for $U_{1 \text { conv }}$}

$U_{1 \text { conv }}$ is the mean convective velocity of the fluid in a CVP vortex at $s_{v 1}$, in the direction tangent to its trajectory. 
One difficulty in the estimation of $U_{1 \text { conv }}$ is knowing $s_{v 1}$. Recall that $s_{v 1}$ is the point along a CVP vortex trajectory coincident with $\Gamma_{1}$, the vortex maximum circulation. From figures 6.2 and 6.3 , it is clear that there is no general agreement on $s_{v 1}$ among previous experiments and analyses. In general, however, $s_{v 1}$ increases as $V R$ increases. This is as would be expected; at larger velocity ratios, the distance for the jet to align itself with the crossflow is longer, and therefore the counterrotating vortices develop over a longer distance. Figure 6.6 shows averages and ranges for $s_{v 1}$ at various velocity ratios; these average values and ranges are based on data from the six references included in figures 6.2 and 6.3. Note that for some of these references, the lowest $s_{v}$ included in the measurements is taken to be $s_{v 1}$; the more correct $s_{v 1}$, however, may actually be somewhat lower than reported.

To obtain an estimate for $U_{1 c o n v}$, assume that the counterrotating vortex pair is significantly developed by the end of the potential core of the deflected jet. (Considering that the end of the potential core is reached at approximately $s_{v} / D_{j}=5$, this assumption is most pertinent to intermediate velocity ratios, as can be deduced from figure 6.6.) For a simple free jet, the average velocity of the jet near the end of its potential core is about $U_{j} / 2$ (see figure 6.7). Assume also that the jet has done a significant portion of its bending by the end of the potential core, so that there is an additional outerflow of approximately $U_{c f}$, as is shown in figure 6.7. Consider the mean convective velocity of the bound vortices to be an average between the average (free) jet velocity $\left(\approx 1 / 2 U_{j}\right)$ and the outerflow velocity $\left(\approx U_{c f}\right)$. This yields an estimate for $U_{1 c o n v}$

$$
U_{1 \mathrm{conv} v} \approx U_{j} \frac{V R+2}{4 V R}
$$

The solid line in figure 6.8 shows this result. 
$U_{1 \text { conv }}$ data from the literature are scarce, but those which are available in the literature are shown along with equation 6.16 in figure 6.8. For these data, the locations of measurement are given in terms of $X / D_{j}$ only. Those values are indicated next to the data points. The corresponding values for $s_{v} / D_{j}$ depend on $V R$. Note, therefore, that all the data don't necessarily correspond to locations at which maximum circulations were also measured, but they do coincide fairly closely to where they would be expected, based on figure 6.6.

Also included in figure 6.8 are new data measured with a total pressure Kiel probe along $s_{v}$. The method by which these estimates for $U_{1 \text { conv }}$ were deduced from the total pressure measurements is given in appendix $B$. The trajectories of the CVP vortices required for these measurements were estimated from flow visualizations which used smoke to seed the jet fluid, e.g., figure 3.4.

Considering the approximations incorporated in this analysis and the uncertainty of values for $s_{v 1}$, the use of equation 6.16 appears acceptable.

\subsection{Present result and comparison with others}

By combining equations 6.16 and $6.15 \mathrm{a} \& \mathrm{~b}$, the predicted circulation for one vortex of the counterrotating pair is therefore obtained;

$$
\Gamma_{1} \approx \frac{\pi V R D_{j} U_{j}}{V R+2}, \quad \Gamma_{1} \approx \frac{2 V R D_{j} U_{j}}{V R+2}
$$

Nondimensionalizing, the results of this analysis are given by

$$
\gamma_{1} \approx \frac{\pi V R^{2}}{2(V R+2)}, \quad \gamma_{1} \approx \frac{V R^{2}}{V R+2}
$$

Experimental data (Fearn \& Weston 1974; Thompson 1972) generally indicate that the CVP vortex strength, $\gamma_{1}$, increases approximately linearly with $V R$. Fearn \& Weston note that $\gamma_{1} \approx .7 V R$ while the analysis of Nunn (1985) uses 
$\gamma_{1} \approx 625 V R$. The present results, given by equations $6.18 \mathrm{a} \& \mathrm{~b}$, predict that the dependence of $\gamma_{1}$ is nearly linear with $V R$ but not exactly so.

In figure 6.9, the present results are compared with previous analytical and experimental results. These data from the references are believed to be representative of $\gamma_{1}$ for each reference, within the limits of the completeness of the published data.

Both results (equations $6.18 \mathrm{a} \& \mathrm{~b}$ ) show that $\gamma_{1}$ increases nearly linearly with $V R$. This is in general agreement with the analytical and experimental results of others. That the circulation in a CVP vortex increases with increasing $V R$ is consistent with the premise of Broadwell \& Breidenthal (1984), that to the far field, the jet looks like a point source of $Z$ momentum, equivalent to a lift force producing a counterrotating vortex pair. Since the lift force increases with increasing $V R$, it follows that the strength of the counterrotating vortices $\left(\gamma_{1}\right)$ increases also.

The circulation estimated when matching the total vorticity flux from the nozzle $\left(\Phi_{j}\right)$ with $\Phi_{1}$ (case (a), equation $6.18 \mathrm{a}$ ) is larger than all other results in figure 6.9. When only the flux of $X$ vorticity from the nozzle $\left(\Phi_{j, x}\right)$ is used to match $\Phi_{1}$ (case (b), equation 6.18b), the estimated CVP vortex circulations are much closer to the experimental and analytical results of others. These results follow the expectation that the use of the total vorticity flux from the nozzle yields an upper limit to the vortex pair strength. The better estimate provided by equation $6.18 \mathrm{~b}$ suggests that the counterrotating vortex pair may evolve primarily from the flux of streamwise $(X)$ vorticity from the jet nozzle.

That the analysis gives a reasonable estimate for the circulation of a CVP vortex suggests that the hypothesis is correct. In particular, the analysis suggests that the source of CVP vorticity is vorticity issuing from the jet nozzle. The results support the premise that vorticity issuing from the nozzle evolves into the 
counterrotating vortex pair and that the evolution occurs within the near field. 


\section{Chapter 7}

\section{Discussions and summary}

In chapter 5, experimental results are presented which show that the source of the wake vorticity is the crossflow boundary layer. Additional measurements characterizing the wake more fully are also included. Chapter 6 addresses the near field development of the counterrotating vortex pair of the jet. Here, more general discussions addressing the transverse jet are presented.

\subsection{The misunderstood wake}

The wake of the transverse jet has been misunderstood in the sense that it has been described as vorticity shed from the jet in manner analogous to shedding from a solid obstacle. It has been shown that this is physically incorrect. In particular, since the jet does not present the crossflow with a solid surface at which to generate vorticity, the vorticity for the wake structures must have been generated somewhere else.

Even with the very different formation mechanisms for solid cylinder wakes and for wakes of transverse jets, there are some features which are similar in the two wakes. Visually, both wakes are comprised of fairly well-defined vortices of opposite signs, the wake of a cylinder being in the familiar pattern of a Karman vortex street. The vortices in the wake of a jet are not so ordered, in general. 
They are also very dependent on the relative strength of the jet to the crossflow, not just the crossflow velocity. Additionally, the axial flow along the vortices in the wake of the transverse jet is greater, due to entrainment by the deflected jet. Structures of both wakes convect with characteristic frequencies. While $S t_{w}$ for circular cylinders near the present Reynolds numbers are in the range of .20 to .21 , those for wakes of transverse jets are found to be somewhat lower for most velocity ratios. Even though cylinders provide solid surfaces while jets provide fluid and entraining surfaces, both are obstacles to the crossflow. It is this similarity, along with the qualitative similarities listed previously, which have likely contributed to the misunderstanding of wakes of transverse jets.

Additional to the similarities between the two types of wakes, the following comments address possible issues which have also contributed to the misunderstanding.

\subsubsection{Kinematics vs. dynamics}

It is important to distinguish between the presence of vorticity and the generation of vorticity. Clearly, the presence of vorticity at some point in the flow does not imply that it was newly generated there. Vorticity convects, stretches, turns, and diffuses to reach regions of the flow away from walls. A kinematics viewpoint considers the velocity field or, equivalently, the vorticity field; vorticity is present where the velocity gradients are such to provide for nonzero vorticity. For example, consider the near field region of the transverse jet schematically indicated in figure 7.1. A vorticity component in the $Z$ direction $(\varsigma)$ near the region indicated by the arrow is expected. Afterall, $\zeta=(\partial V / \partial X-\partial U / \partial Y)$ where $\partial V / \partial X$ is expected to be relatively small compared to $\partial U / \partial Y$ near that location. Since neither the crossflow boundary layer nor the nozzle boundary layer initially contain nonzero 
$\zeta$, this component of vorticity, located by kinematics, may be too readily considered newly generated vorticity. Through the analogies to the flow about circular cylinders, the location indicated in figure 7.1 is an area where vorticity for the wake hăs allegedly been generated at and then shed from. This need not be the case, and is not the case for a uniform density flow.

The dynamics viewpoint concerns itself with the source of vorticity and its subsequent motion; where does the vorticity come from? The approach taken here addressed the dynamics of the vorticity in the wake structures.

Solely looking at the flow kinematically not only can be misleading for the description of the source of vorticity for the wake, but also for the description of the development of the counterrotating vortex pair of the jet. Just as vorticity for the wake is not generated at the location indicated by the arrow in figure 7.1, vorticity for the counterrotating vortex pair is also not generated there. The development in chapter 6 suggests that the vorticity from within the nozzle evolves into the CVP vorticity.

\subsubsection{Rate of strain vs. vorticity}

Although there is no mechanism in the present flow by which to generate new vorticity within the flow, such is not the case for the rate of strain (Morton 1984). The 2-D equation governing the rate of strain for a Newtonian, barotropic, isothermal, and incompressible flow is given by

$$
\frac{D \varepsilon}{D t}=-\frac{2}{\rho}\left(\frac{\partial^{2} p}{\partial Z \partial X}\right)+\nu \nabla^{2} \varepsilon
$$

where $\varepsilon$ is the rate of strain. The term with pressure $p$ is a legitimate source term for new rate of strain. Therefore fresh rate of strain can be generated within a flow, away from solid boundaries. Confusion could arise because curved streamlines can 
result from either the presence of vorticity (consider solid body rotation) or rate of strain production (consider the deflection of streamlines near a bluff body obstacle or the potential flow around a circular cylinder). The appearance of curved streamlines, such as the deflected streamlines around the jet in a crossflow (see, e.g., several photographs in chapter 3 ), does not necessarily imply the presence nor generation of vorticity.

\subsubsection{Total pressure gradients}

A total pressure gradient such as the one between a jet and its crossflow does not generate fresh vorticity. To illustrate the point, consider the development of a simple plane shear layer, sketched in figure 7.2. Boundary conditions at large $+Y$ and $-Y$ provide a total pressure gradient in $Y$ for all $X$ within the shear layer. Even though diffusion and vortex interactions change the vorticity distribution

across the shear layer, the net circulation per unit length in $X$ is $\left(U_{1}-U_{2}\right)$ for all $X$, such as at $X_{a}$ and $X_{b}$ in the drawing; the total pressure gradient across this homogeneous shear layer does not generate new vorticity. All the vorticity in the shear layer originated on the upper and lower surfaces of the splitter plate, at $X \leq 0$. This point can be extended to the case of the jet issuing into a crossflow.

\subsubsection{Guideline for vorticity generation}

To determine whether new vorticity is being generated, or, equivalently, whether circulation is being added, it is best to consider a fundamental physical mechanism by which vorticity is produced. As is pointed out by Morton (1984), there must exist a relative tangential acceleration across adjacent layers of fluid in order for vorticity to be produced. Such a relative tangential acceleration cannot occur within a homogeneous fluid. A pressure gradient, for instance, accelerates 
adjacent layers of fluid equally. However, for flows with density gradients or for layers of fluid adjacent to walls, pressure gradients do set up relative tangential accelerations.

\subsection{Dependence of the wake on $V R$}

The following list is a summary of what has already been discussed in chapters 3 and 5 regarding the wake effects of changing the jet to crossflow velocity ratio:

1. In section 3.3, photographs with the smoke-wire at $X / D_{j}=2$ and $Y / D_{j}=$ 0 show that there is reverse flow in the near wall, very near wake when $V R=2$, 8 , or 10 . No such reversal of flow is observed when $V R=4$ or 6 .

2. Photographs of the wake vortices in side view show their dependence on $V R$. Puff-like wake structures are visualized at $V R=2$ while very coherent wake vortices occur at $V R=4$. From $V R=6$ through $V R=10$, the wake structures tend toward thinner strand-like structures which extend from a densely structured region near the crossflow wall to the deflected jet.

3. Cross sectional views of the wake vortices indicate that the widths of the wake vortices for $V R=2,4 \& 6$ are roughly uniform along their spans. However, the wake vortices appear to get thinner with distance from the crossflow wall for $V R=8 \& 10$.

4. In section 5.2, $\overline{\theta_{w}}$ and $\overline{\delta_{w}}$ (average is over several downstream locations) are shown to increase with increasing distance from the crossflow wall for $V R=$ 4. For $V R=8, \overline{\theta_{w}}$ and $\overline{\delta_{w}}$ remain roughly unchanged in $Z / D_{j}$.

5. The total pressure deficit in the wake also increases with increasing $Z / D_{j}$ for $V R=4$, while it is found to generally decrease and level off for $V R=8$.

6. The coherence and positions of crossflow boundary layer separation events 
vary with $V R$, as shown in section 5.1 . They are most distinct near $V R=4$.

7. $S t_{w}$ is very dependent on $V R$, regarding both the value and sharpness of the power spectral peak for $f_{w}$. The most compelling result here is that the $f_{w}$ spectral peak is sharpest near $V R=4$ and that $S t_{w}$ is independent of $R e_{c f}$ and all other parameters near $V R=4$.

\subsubsection{More on $V R$ effects}

To clarify and expand on the dependence of $S t_{w}$ on $V R$, consider figure 7.3, which shows wake Strouhal numbers for $R e_{c f}=3800$ and $L_{j} / D_{j}=5$ only. The plot is divided into 5 regimes: $V R \approx 2, V R \approx 3, V R \approx 4, V R \approx 6$, and $V R>6$. These five regimes are coincident with variations in the wake appearance and measurements as $V R$ is changed. This aspect is addressed in detail in what follows.

Figure 7.4 shows side views of the wake vortices for six different velocity ratios. As before, the wake structures are visualized here by seeding the crossflow boundary layer with smoke, i.e., $Z_{s w} / D_{j}=0+$. In order to get a better feel for the dependence on $V R$, three typical photographs for each $V R$ are shown. These six velocity ratios were chosen to complement the $S t_{w}$ results shown in figure 7.3 ; each regime is represented.

At $V R=2.1$ (figure $7.4 \mathrm{a}$ ), the wake structures are fairly diffuse "puffs" very close to the crossflow wall. As $V R$ increases to 3 (figure 7.4b), the jet bends further away from the wall and a clear change has occurred in the appearances of the wake structures. The structures are thinner, more defined, and are greater in number. Note, in figure 7.3 , that the power spectra at this velocity ratio have a very poor peak. The vortices are very coherent, and more evenly spaced at $V R=4$ (figure 7.4c). This corresponds to sharp spectral peaks near $V R=4$. The photographs in figure $7.4 \mathrm{~d}$ show the wake at $V R=5.8$, the second regime with a discontinuity. At 
$V R=6.6$ (figure 7.4e) the wake has much more disorder and scattered structures, corresponding to a very poor spectrum in figure 7.3. There are locations where one would expect a vortex, but there is none. Finally, figure 7.4f shows photographs of the wake at $V R=8$. Characteristic of the higher velocity ratios, most of the smoke from the separated boundary layer remains close to the crossflow wall. Only very thin strands of smoke connect the majority of the separated boundary layer fluid near the wall with the jet.

Photographs of $V R=4$ and $V R=8$ wakes can be correlated with the changes in $\delta_{w}$ and $\theta_{w}$ with distance from the crossflow wall. Recall the results of figures 5.36 and 5.37 , which show that a relatively larger portion of the wake mass and momentum losses at $V R=4$ were found several diameters from the crossflow wall. The losses are roughly evenly distributed with $Z / D_{j}$ at $V R=8$. This behavior of the $V R=4$ and 8 wakes is manifested in the photographs. The wake structures at $V R=8$ (figure 7.4e) are significantly thinner further from the wall than they are at $V R=4$ (figure 7.4c). Similarly, the photographs at $V R=4$ and 8 also correlate well with the total pressure deficits measured in the wake. Recall that for $V R=4$, the total pressure losses increased with distance from the wall while they decreased for $V R=8$. The visual thicknesses of the wake vortices appear to be good indications of how much boundary layer fluid is present.

Figure 7.5 shows the near wall, very near wakes of transverse jets issuing at the viewer. As before, the smoke begins in the crossflow boundary layer. Again, the appearances of the structures follow the changes in $S t_{w}$ with $V R$. At $V R=2$ (figure 7.5a), the very near wake is relatively "open", with the legs of the horseshoe vortices clearly seen. As $V R$ is increased to 3 (figure 7.5b), the near wake closes, and at $V R=4$ (figure $7.5 \mathrm{c}$ ) the separation events are most clearly seen. Above $V R=6$, the appearance of the near wall, very near wake changes substantially, 
as is exemplified by figure $7.5 \mathrm{~d}(V R=8)$.

To further illustrate the wake dependence on $V R$, all photographs showing the near wall $\left(Z_{s w} / D_{j}=0+\right)$, very near wake $\left(X / D_{j} \leq 5\right)$ were compiled to classify this portion of the flow as symmetrical or asymmetrical about $Y=0$. For example, the photographs in figure $7.5 \mathrm{c}$ show essentially asymmetrical very near wakes since the separation events and nascent wake structures are arranged in a staggered pattern, more like those in a Karman vortex street. The photographs in figure $7.5 \mathrm{~d}$ show the very near wake to be essentially symmetrical about $Y=0$. Considering 20,52, 50, 19, and 18 photographs for $V R=2,4,6,8$, and 10, respectively, the near wall, very near wakes for $V R=2,8$, and 10 are found to be generally symmetrical while those for $V R=4$ are asymmetrical. For $V R=6$, the photographs are inconclusive.

To summarize, the wake structure is very dependent on the jet to crossflow velocity ratio. Specifically for $R e_{c f}=3800$, a variety of photographs of the wake vortices, wake frequency measurements, wake velocity profiles, and wake centerline total pressure measurements combine to show that there is a discontinuity in the wake structure near $V R=3$, that there is something special about the wake near $V R=4$, and that there is another discontinuity in wake structure around a velocity ratio of 6 . The appearance of the wake is thereby different for $V R>6$.

\subsubsection{Why are $V R \approx 4$ wakes special?}

Certainly the characteristics of the wake near $V R=4$ are very striking. The photographs and spectral measurements of the wake and its associated separation events all indicate that the wake of the transverse jet is most coherent or ordered near $V R=4$. This was found to be true for the full range of Reynolds numbers considered $\left(3800 \leq R e_{c f} \leq 11400\right)$. Recall, also, that $S t_{w}$ does not vary with $R e_{c f}$ 
nor with $L_{j} / D_{j}$ and $\delta_{c f} / D_{j}$ near $V R=4$, as it does in general.

Why are $V R \approx 4$ wakes so special? It is proposed that the proximity of the deflecting jet to the crossflow wall is an important factor. The model proposed for the wake formation in chapter 5 requires the jet to entrain the closest portion of the separation event structure away from the wall. As the vorticity from the crossflow wall is entrained by and convected with the jet, a connection between the wake vortices and the jet is established. The other end of the wake structure remains attached with the vorticity in the crossflow boundary layer. It is conjectured that, at some $V R$, the ability of the jet to entrain and affect the crossflow fluid which has separated diminishes as the jet is further removed from the crossflow wall.

Consider the drawings in figure 7.6. Figure 7.6 shows outlines of jet trajectories at three values of $V R$ as determined from the smoke visualizations in figure 3.4. The arrows in the sketches indicate an idealized entrainment flow pattern in the $Y=0$ plane. (Recall that for a circular jet issuing from a wall, the entrained flow is in a direction perpendicular to the jet centerline.) At the low velocity ratios such as $V R=2$, the jet remains very close to the crossflow wall. Even though its entrainment at the wall is felt very strongly, the close proximity of the jet to the wall makes it difficult to distinguish between jet and wake fluid. In a sense, the jet is too close to produce well-defined wake structures, and the jet is not cleanly separated from the wall. Near $V R=4$ (figure $7.6 \mathrm{~b}$ ), the jet is now far enough from the wall to induce significant turning of the separated boundary layer vorticity, but it is close enough to strongly and efficiently pull the separated fluid away from the crossflow wall. This intermediate distance between the deflected jet and the wall allows for some stretching of the wake vortices as they form, thus defining them even more. At larger velocity ratios like $V R=8$ (figure 7.6c), the crossflow boundary layer, although separated, is not easily nor efficiently entrained by the 
jet, for the distance of the jet from the near wake wall region is now larger. This is quite evident in the flow visualizations of the wakes at large velocity ratios. The side views of figures $3.11 \mathrm{~d} \& \mathrm{e}$, e.g., show that the majority of smoke (marking the boundary layer fluid) remains relatively close to the wall, with only thin smoke strands extending to the jet.

These three situations, as just described, correlate quite well with the flow visualization. They also allow for the transitions in $S t_{w}$ outlined in the previous section. The wakes of $V R=2$ and $V R=4$ jets are both, in a sense, stable situations. The discontinuity observed near $V R=3$ occurs between the jet not pulling much crossflow boundary layer fluid from the wall ( $V R \approx 2$ case) and the jet very efficiently entraining the crossflow boundary layer fluid ( $V R \approx 4$ case). Furthermore, the discontinuity noted previously on several occasions near $V R=$ 6 appears to coincide with the $V R$ at which the jet become too removed from the crossflow wall to efficiently form wake structures.

Complementary to these entrainment effects of the jet proximity to the crossflow wall is the trajectory of the entrained fluid with the jet. At the higher velocity ratios, where the jet is more vertical, the entrained crossflow fluid follows a trajectory which first takes it nearly vertical, relatively far from the crossflow wall, before it turns substantially into the crossflow direction. Conversely, for velocity ratios near four, the entrained fluid quickly is turned in the crossflow direction. The effect of this on the coherency of the wake is two-fold. First, the geometry of the situation allows for the wake structures to remain mostly independent of the jet structure. Second, the close proximity of the jet allows for continual entrainment of boundary layer fluid along the developing wake vortices. 


\subsection{What sets $f_{w}$ ?}

It is well known that shear layer flows of all sorts, including circular cylinder wakes, have characteristic frequencies or modes associated with them. As has been shown, the wake of the transverse jet is no different in this respect. It is of interest now to address possible mechanisms which are responsible for setting the wake frequencies. Since the wakes for $V R \approx 4$ jets are the most coherent, they are regarded with relatively more detail.

\subsubsection{Cylinder wake modes}

Although the mechanism by which the wake vortices of the transverse jet form is very different from the mechanism for solid cylinder wakes, it is in the interest of perspective to first discuss the frequency modes inherent to cylinder wakes. For cylinders at Reynolds numbers close to the present values, several modes encompassing the near and far wakes exist, each with its own characteristic frequency. First, there is a Kelvin-Helmholtz roll-up of the shear layer separating from the body. The characteristic frequency of this shear layer scales with the velocity difference across the layer and its thickness. The familiar Karman vortex street then develops within a few cylinder diameters, and with it, its characteristic shedding/wake frequency. That $S t_{w} \approx .20$ to .21 for crossflow Reynolds numbers in the thousands is not fully understood. It is possible that the characteristic frequency for the Karman street is determined by a combination of the unsteady boundary layer separation from the body and the stability of the near wake mean velocity profile (Cimbala 1984). Cimbala found that a far wake structure also develops behind solid cylinders, beyond where the Karman vortex street dissipates. The mechanism for this structure was found to be due to an instability of the mean 
far wake velocity profile.

\subsubsection{Possible transverse jet modes}

Since the shear layers separating from a cylinder undergo a Kelvin-Helmholtz instability, it is possible that an analogous instability and roll-up of the separating crossflow boundary layer near the orifice of the jet exists. Each separation event appears to lead to one vortex roll-up. A series of vortices along a separating stream surface normally associated with Kelvin-Helmholtz instabilities was generally not observed. This is possibly due to the effects of the jet, which are felt too early for subsequent roll-ups to occur.

Particularly near $V R=4$, the alternating separation events from one side of the jet to the other appear somewhat like alternating shedding from the crossflow wall. Compared to shedding from cylinders, however, the problem here is complicated considerably by the fully three-dimensional flow at separation. In any case, a preferred wake mode may exist which scales in a similar fashion to the Karman vortex wake mode.

One reason that the situation for transverse jets is more complex than for cylinders is that the formation of the wake vortices of the jet may be affected by natural modes associated with the jet. In the case of cylinders, if flow-induced vibrations are eliminated, there is no analogous forcing of or competition with the wake.

There are two relevant frequency modes associated with the transverse jet itself, both of which are common to free jets also. The first is the mode associated with the instability of the distorted shear layer resulting from separation from the jet orifice. The most amplified frequency of this shear layer, at least for a free jet, scales with the jet boundary layer initial momentum thickness, $\theta_{j}$, and the jet 
velocity. Values for $S t_{\theta j}$ in the range of .009 to .018 appear in the literature (see Gutmark \& Ho 1983, table IV, for a compilation of these references).

Additionally, there is a larger scale mode, referred to as the jet "preferred mode" or "puff" mode, near the end of the potential core of the jet. The frequency of this mode is normally scaled with $D_{j}$ and $U_{j}$. Strouhal numbers associated with this mode, $S t_{j}$, have been measured in the range from .24 through .64 . These frequencies have been measured anywhere from 3 to $6 D_{j}$ from the nozzle exit. Again, a complete listing of these references is in Gutmark \& Ho (table I).

Measurements of Strouhal frequencies were made along the leading edge of the bending jet. The results are shown in figure 7.7. (For comparison, the Strouhal frequencies along the potential core of a free jet at the same Reynolds number are shown in figure 7.8.) As expected, the shear layer frequencies decrease with distance along the jet, at least partially due to the vortex merging process. Peaks in the power spectra were not discernable beyond $Z / D_{j} \approx 5$. From these measurements, the preferred mode of the transverse jet has $S t_{j} \approx .2$. (That measured for the free jet is $S t_{j} \approx .38$, which is in the range quoted above for free jets.)

The subsequent evolution of the wake of the transverse jet and its stability in the far field is still very much an open question. Kuzo \& Roshko (1984) have observed a wake several hundred jet diameters downstream from the jet. Whether the frequencies remain the same, whether vortex merging occurs, and whether a new instability takes root are all issues of interest. Assuming that the far field coherent wake structures observed by Kuzo \& Roshko are those from the near field, such a lifespan for the wake vortices is unique compared to Karman vortices. It is conjectured that the continuous stretching of the wake and feeding by the crossflow boundary layer is instrumental to this. 


\subsection{Entrainment and mixing}

With the new wake formation mechanism and the analysis regarding the counterrotating vortices, it is timely to discuss the results' implications on entrainment and mixing processes of transverse jets.

There are various definitions of entrainment and mixing. Roshko (1976) defines entrainment as the "incorporation of nonturbulent, usually irrotational fluid into the turbulent region". That this process is dominated by the large-scale structure of the shear flow has been discussed already in chapter 1. Additionally, mixing occurs at smaller scales of turbulence and due to molecular diffusivity. Here, entrainment is used in the sense that it is a subset of the whole mixing process, which encompasses the large-scale process (entrainment) and the smaller scale processes of viscous and molecular diffusion. Entrainment refers to the "incorporation" or "induction" of external fluid into the shear layer of interest due to the large scale structures, such as those observed in this study.

Entrainment is not restricted to the incorporation of irrotational fluid, as is generally the case when referring to plane shear layers, for instance. The external fluid need not be irrotational nor nonturbulent for it to be entrained into a shear layer. This is particularly evident for the wake vortices. The wake vortices, certainly rotational and turbulent, are formed as a portion of each separation event is pulled away from the crossflow wall by entrainment of the jet.

The present results can be used to discuss the entrainment processes of transverse jets. They do not directly show the mixing processes at the smaller turbulent and molecular scales. For the Reynolds numbers of concern here, however, it appears that the mixing process is limited by the larger scales, i.e., by entrainment. This is discussed by Broadwell \& Breidenthal (1984) in more detail. They found 
that the mixing in transverse (water) jets was independent of Reynolds number beyond a critical value for the local far field Reynolds number $R e_{l o c}$.

$$
R e_{l o c}=\frac{\Gamma}{\nu}=\left(\frac{\pi}{4}\right)^{1 / 2} \frac{U_{j} D_{j}}{\nu}\left(\frac{l^{*}}{X}\right)^{1 / 3}
$$

Their rough estimate for the critical value of $R e_{\text {loc }}$ is 300 . For the "worst case" or minimum $R e_{c f}$ and $V R$ studied here, this corresponds to an $X / D_{j} \approx 20,000$. Accordingly, then, the large scale structures, responsible for entrainment, are also responsible for the overall mixing rate in the flow up to approximately 20,000 jet diameters downstream of the orifice! Therefore, it is pertinent to discuss the roles of the counterrotating vortices and wake vortices in mixing.

\subsubsection{Transverse jets make better mixers}

As has been seen, the flow fields of transverse jets are very different from free jets. Even with the same "initial" jet conditions (including jet velocity, jet vorticity flux, etc.), the flows are subject to very different viscous and pressure forces beyond the nozzle. In fact, the effects of the crossflow are most likely already felt within the nozzle. The numerical results of Coehlo \& Hunt (1989) suggest this. With these differences, what effects do crossflows have on the mixing of jets?

The results of Kamotani \& Greber (1972) and Broadwell \& Breidenthal indicate that a transverse jet is a better mixer of ambient and jet fluid than is a free jet. Kamotani \& Greber measured the temperature decay in a heated transverse jet with distance from the nozzle. Their figure 15 (reproduced here as figure 7.9) shows that the temperature decay is faster for a transverse jet than for a free jet. One interpretation of their result is that the entrainment of cold ambient fluid is enhanced for the case of the transverse jet, leading to better mixing and a quicker temperature decay. 
The results of Broadwell \& Breidenthal also show better mixing for transverse jets. Their experiments involved the injection of alkali jets with phenolphthalein into acidic crossflows. They were able to measure flame lengths from the $\mathrm{pH}$ indicator, phenolphthalein. Figure 7.10, which plots flame length against $V R$ for several volume equivalence ratios, shows their data for transverse jets and data from Weddel (see Hottel 1953) for free jets (except $\phi=1.1$ ). (The volume equivalence ratio, $\phi$, is the ratio of ambient to injected fluid required to turn the phenolphthalein from red to clear in color.) These results show that flame lengths for transverse jets are, in most cases, significantly lower than for free jets, at the same equivalence ratios. Therefore the transverse jets can be considered better mixers than free jets. (Note that the results of Broadwell \& Breidenthal all are in excess of the critical local Reynolds number of 300 at the flame tips.)

Broadwell \& Breidenthal speculate that the counterrotating vortex pair may somehow be responsible. Is it also possible that the wake vortices play a role?

\subsubsection{The role of the wake}

Since the source of fluid for the wake vortices is fluid in the crossflow boundary layer, the wake structures don't directly contribute to the enhanced mixing of transverse jets. A significant portion of the wake is crossflow fluid, either ambient fluid (crossflow fluid outside the boundary layer) or boundary layer fluid. The wake vortices may still play an important role in mixing within the deflected jet, indirectly. Since one "end" of each wake vortex extends into the jet, the vorticity field from that portion of each structure may act as a stirrer within the jet and thereby enhance its mixing.

It would be of interest to remove the wake and note the effects, if any, on the flame lengths. It is relevant to note that both the results of Kamotani \& 
Greber and of Broadwell \& Breidenthal do not make mention of wakes behind their transverse jets. In each case, however, the jet issued through a wall with the nozzle mounted flush with the wall. One can only assume that a wake was present in both cases.

Although the effects of the wake vortices on mixing within the jet cannot be easily known from the present results, the results do strongly suggest that it may be feasible to efficiently mix fluid near or in the crossflow boundary layer with the ambient crossflow by taking advantage of the wake formation dynamics. Flow visualization such as that shown in figure 3.12 indicates that the wake vortices (composed of crossflow boundary layer fluid) entrain ambient crossflow fluid. The streakline patterns indicate this.

\subsubsection{The role of the counterrotating vortex pair}

The decrease in the transverse jet flame length correlates well with the increase in $\Gamma_{1}$ as $V R$ is increased. To see this, compare figure 7.10 with figure 6.9 . If the circulation of a vortical structure is greater, then too is its induced velocity field contributing to entrainment. (Consider, for instance, the Biot-Savart law.) Its entrainment (and therefore mixing) is enhanced. This indicates that the counterrotating vortex pair structure may be an important element to the enhanced mixing of transverse jets.

Note that the flame lengths for the experiments of Broadwell \& Breidenthal put the flame tips in the far field of the flow. Even then, the near field entrainment and vortex strengths are relevant. Results of others (see chapter 6) indicate that the counterrotating vortex pair circulations are larger for larger velocity ratios both in the near fields and far fields. Also, the wake vortices exist both in the near field and far field. 
One must also consider that perturbations to the flow in the near field can affect the far field state of the flow. Therefore, the near field structure and dynamics likely have an effect on mixing not only in the near field but also in the far field.

\subsection{Variations on the theme}

There are many variations to the transverse jet flow studied here, both in regard to the physical set-up and to the flow conditions. Some of them, along with their possible implications, are discussed here.

\subsubsection{Protruding jet}

It is of interest to compare the wake structure here to the corresponding case of a jet issuing from a pipe protruding into the crossflow. In the latter case, the experimental results of Moussa et al. (1977) show that the wake Strouhal numbers of transverse jets more closely match the Strouhal numbers of cylinder wakes. In addition, they observe that the wake spectral peaks are generally sharper for the case of a protruding jet than they are for the flush jet. For the protruding jet, they state that "shedding from the jet ... is dominated by shedding from the solid pipe." More precisely, it is consistent with the present case to speculate instead that the vortices in the wake of a protruding jet are extensions of the vortices shed from the pipe, just as the vortices in the wake of a flush-mounted jet are extensions of vorticity from the crossflow boundary layer. 


\subsubsection{Crossflow boundary layer alterations}

Due to the importance of the crossflow boundary layer for the wake, it is also of interest to speculate on the effects of changing the characteristics of that boundary layer. In chapter 5 , the effects of changing $\delta_{c f}$ are discussed, but the approaching boundary layer remained laminar in those cases. If the boundary layer were turbulent, what would the effects on the wake be? It is well known that a turbulent boundary layer is more resistent to separation. It follows that the separation events at the crossflow wall may move further behind and downstream of the jet. The area of the effective separation region would then decrease. The size of the separation region, determined by the locations of separations events, may subsequently affect $S t_{w}$.

For all cases studied, $\delta_{c f}<D_{j}$. The situations where $\delta_{c f} \approx D_{j}$ and where $\delta_{c f}>D_{j}$ would also be of interest. For an extreme case, $\delta_{c f} \gg D_{j}$, the jet would not escape the crossflow boundary layer at all. The jet would then be injected into a crossflow with shear. The other extreme is where the crossflow boundary layer is removed altogether. If there were no crossflow boundary layer, the present results suggest that there would be no wake vortices. The subsequent effects on the trajectory and mixing of the jet would then also be of interest.

\subsubsection{Jet alterations}

Here and in most other studies, the jet has an approximately top hat velocity profile. A transverse jet with a fully-developed pipe flow or a Hagen-Poiseuille exit velocity profile may have different flow characteristics. The effects, however, are certainly not obvious. For instance, if one considers a jet whose velocity profile at the nozzle exit is of the fully-developed pipe flow variety and whose mean exit 
velocity is equal to that of the top hat jet, the flux of $X$ vorticity from the orifice of the pipe flow jet is about twice as large as the corresponding top hat jet. As in chapter 6,

$$
\Phi_{j, x}=\left|\int_{0}^{\pi} \int_{0}^{D_{j} / 2} r \frac{d W}{d r} W(r) d r \sin \theta d \theta\right| .
$$

For fully developed pipe flow,

$$
W(r)=U_{j \max }\left(1-\frac{4 r^{2}}{D_{j}^{2}}\right) \quad \text { and } \quad \bar{U}_{j}=\frac{U_{j \max }}{2}
$$

Therefore

$$
\Phi_{j, x}=\frac{16 \bar{U}_{j}^{2} D_{j}}{15}
$$

for a pipe flow jet, compared to $U_{j}^{2} D_{j} / 2$ for a top hat jet. The effect of the larger $X$ vorticity flux from the orifice would seem to predict stronger counterrotating vortices, following the reasoning developed in chapter 6. But due to the closer proximity of $X$ vorticity of opposite signs, a ramification of the pipe flow velocity profile, cancellation of vorticity between the two sides appears more likely, particularly in regards to cross diffusion of vorticity. The effect of this would be to weaken (lower the circulation in) the counterrotating vortices. In fact, if the far field, asymptotic behavior of the jet is indifferent to the initial profile of the jet for jets of equal average exit velocities, these two competing effects may cancel to yield the same vortex pair strength, jet trajectory, etc.

The jet can also be altered by changing its density relative to the crossflow. By adding density gradients to the flow, new vorticity can be generated at the interface of the jet and the crossflow. The vorticity transport equation now contains a source term for new vorticity, the baroclinic term. A pressure gradient component perpendicular to a density gradient component would generate vorticity. 


\subsection{Summary}

The principal message of this research is the finding that the system of vortices in the wake of a transverse jet is distinctly different in origin and formation from the vortices which are shed from a solid cylinder. In the case of the transverse jet, the jet/crossflow interface cannot generate new vorticity. Theory for sources of vorticity, considered in chapter 4 , restricts the generation of fresh vorticity to solid surfaces for this kind of flow (barotropic, isothermal, and incompressible). In chapter 5 , the combination of using smoke as a vorticity marker, spectral measurements, and wake velocity profiles showed that the wake vorticity comes from the boundary layer on the wall from which the jet issues. The crossflow boundary layer separates near the downstream side of the jet because it cannot negotiate the adverse pressure gradient which is imposed on it by the flow around the jet. The vorticity of the separated boundary layer is then incorporated into the wake vortices, which extend from the crossflow wall to the bending jet.

With this result, the transverse jet cannot truly be considered a free shear flow. The influence of the crossflow wall, if the jet is mounted flush with it, must be considered. The presence of the crossflow wall directly contributes to the presence of the wake vortices. In turn, the wake vortices may significantly affect the jet dynamics, including the counterrotating vortex pair. Even for the case of a jet whose supply pipe protrudes into the crossflow, the vortices shed from the protruding pipe must be accounted for.

The near field development of the counterrotating vortex pair, the jet structure which has dominated much of transverse jet research, was addressed in chapter 6. It is hypothesized that the flux of vorticity from the jet nozzle comprises the flux of vorticity in the counterrotating vortex pair of the jet. The hypothesis tests 
well, for reasonable estimates for the circulation in each vortex of the pair are obtained. In particular, the results suggest that the source of vorticity for the vortex pair is the vorticity emanating from the nozzle.

Smoke-wire flow visualization proved to be invaluable in showing the near field structure of the transverse jet. The photographs shown in chapter 3 and elsewhere provide various views of the four types of coherent vortical structures which constitute the near field of the transverse jet: the jet shear layer vortices, the nascent far field vortex pair, the near wall horseshoe vortices, and the system of vortices in the wake of the transverse jet. 


\section{Appendix A}

\section{Applications of transverse jets}

In addition to being a fundamental research problem in fluid mechanics, the transverse jet, in its many versions, is also relevant to many technical situations. The applications of transverse jets include, but are not limited to, the following areas: turbomachinery, aerodynamics, waste disposal into the environment, and natural phenomena. Among these applications, there is a variety of flow parameters and physical conditions which define the precise flow. The physical set-up is of importance in terms of the relative angle between the jet and crossflow, the jet exit

geometry, whether multiple jets are used, and whether the jet is positioned such that its exit is flush with one of the walls bounding the crossflow. Flow parameters which are relevant include the relative and absolute velocities and momenta of the jet and crossflow, and the densities of the jet and crossflow. Compressibility may also be an issue. The flow may be multi-phased, and combustion may also occur. The parameters and conditions listed here are not all-inclusive, but they are influential in defining the type of transverse jet one is dealing with.

In turbomachinery, jets of relatively cool fluid are injected at the surfaces of the turbine blades. The use of such a film cooling technique allows for higher turbine inlet temperatures, which are desirable for performance reasons. Strictly speaking, however, the injection for film cooling is not typically transverse. Therefore, in regards to film cooling, the label "transverse" jet is used loosely. Even 
with the use of film cooling, the post-combustion flow within the engine needs to be cooled and made more uniform prior to reaching the turbine stage; for this purpose, combustion chamber dilution jets are used. The goals here are to sufficiently cool the combustion products with the dilution jets and to do so with minimal pressure or aerodynamic losses. While mixing is desirable in the case of dilution jets, film cooling applications aim at separating the hot outer flow from the surface with the injected fluid. Fuel injection also has the characteristics of a jet in a crossflow, such as in the afterburners of gas turbine engines and in supersonic combustion ramjet engines.

The presence of transverse jets can have aerodynamic consequences as well. The exhaust flow for V/STOL aircraft may be oriented at some angle to the flight direction during the transition portion of flight. The interaction between the engine exhaust and crossflow alters the pressure distribution on the aircraft, including possibly its wings and other control surfaces. In addition, this exhaust gas/crossflow interaction may be unsteady. Jets can also be used as aerodynamic controls. For instance, jets of either liquid or gas are used within the nozzles of solid rocket motors for thrust vector control. Also, jets are used for roll control on missiles, have been considered for the control of vortices shed from the forebodies of fighter aircraft, and have been tested for wing tip blowing, for the purpose of improving wing aerodynamics.

The discharge of a variety of pollutants into the environment is often analogous to a jet into a crossflow. Pollutants, including sewage and heated water, are discharged into waterways. Here, the trajectory and mixing of the jet fluid may be of importance. One may need to limit the extent and concentration of polluted (jet) discharge. Similarly, the study of the transverse jet is pertinent to the discharge of pollutants via smokestacks into the atmosphere. 
Occurences in nature often mimic the jet into a crossflow problem. For instance, volcanic eruptions coincident with significant crosswinds have the basic feature of one flow, the volcanic cloud, essentially starting perpendicular to another flow, the crosswind. In addition, the interaction of crosswinds with atmospheric phenomena such as tornadoes and thunderstorms (both with updrafts) may also be natural extensions of the transverse jet flow. 


\section{Appendix B}

\section{Measurement details}

\section{B.1 Crossflow boundary layer thicknesses}

Estimates for $\delta_{c f}$ and $\theta_{c f}$ at $X / D_{j}=0$ are obtained by integrating the appropriate equations using measured boundary layer profile data. The single-wire hot-wire was traversed through the crossflow boundary layer. As an example, consider figure B.1. Shown here are experimental data and the Blasius boundary layer profile which would exist for $L_{j} / D_{j}=10$ and $R e_{c f}=3800$, the situation for the thickest crossflow boundary layer. Note that these are nominal values, meaning that the jet is not present to disturb the crossflow boundary layer.

The standard equations are used to obtain $\delta_{c f}$ and $\theta_{c f}$. In particular,

$$
\delta_{c f}=\int_{0}^{\infty}\left(1-\frac{U}{U_{c f}}\right) d Z
$$

and

$$
\theta_{c f}=\int_{0}^{\infty} \frac{U}{U_{c f}}\left(1-\frac{U}{U_{c f}}\right) d Z .
$$

The experimental data plus the point $(0,0)$ are used to integrate these equations using the simple trapezoidal scheme. For the example shown in figure B.1, $\delta_{c f} / D_{j}=.087$ and $\theta_{c f} / D_{j}=.037$.

Results for all cases are shown in table $\mathbf{2 . 2}$. 


\section{B.2 Jet boundary layer thicknesses at the nozzle exit}

Similar to the case for the crossflow boundary layer, estimates for $\delta_{j}$ and $\theta_{j}$ are obtained experimentally. For convenience, the hot-wire was traversed across the jet shear layer just slightly beyond the lip of the nozzle at $Z / D_{j}=.013$. It is assumed that the thickness of the shear layer at this point is very nearly the same as the thickness of the boundary layer within the nozzle at $Z / D_{j}=0$. Freymuth (1966) (his figure 4) shows that, for Reynolds numbers close to those here, the momentum thickness grows only by a few percent within a distance $Z / D_{j}=.013$. Figure $\mathrm{B} .2$ shows that profile for the separating boundary layer. Again, this represents the thickest jet boundary layer encountered here, and these are nominal values; the crossflow is off.

The experimental data are used to integrate the equations

$$
\delta_{j}=\int_{D_{j}}^{0}\left(1-\frac{W}{U_{j}}\right) d X
$$

and

$$
\theta_{j}=\int_{D_{j}}^{0} \frac{W}{U_{j}}\left(1-\frac{W}{U_{j}}\right) d X
$$

The trapezoidal integration scheme is used. For the sample profile shown in figure $\mathrm{B} .2, \delta_{j} / D_{j}=.045$ and $\theta_{j} / D_{j}=.0093$.

Results for all jet Reynolds numbers are shown in table 2.3.

\section{B.3 Power Spectra Details}

All flow frequencies were obtained using an HP 3582A spectrum analyzer. The analyzer performs a Discrete Fast Fourier Transform on the hot-wire signal with the sampling characteristics shown in table B.1. 
All spectral data used here involved the rms average of $\mathrm{N}$ individual spectra. (All the wake data, for instance, used $\mathrm{N}=32$ ). The rms spectral amplitude $P$ is given by

$$
P=\sqrt{\frac{1}{N} \sum_{i=1}^{N} A_{i}^{2}(f)}
$$

where $A_{i}$ is the amplitude of each discrete frequency for spectrum $i$.

Due to the discrete nature of the spectra (256 points per span), "windowing" is required for a smooth spectra. A Hann window option was selected here, to achieve a compromise between amplitude and frequency accuracy.

The vertical axes for the spectra are given by $\log (P)$, defined as

$$
\log (P) \equiv 10 \log \left(\frac{V_{h w}^{2}}{1 \text { volts }^{2}}\right)
$$

where $V_{h w}$ is the hot-wire signal in volts.

\section{B.4 $U_{1 \text { conv }}$ estimates from total pressure measurements}

Estimates for $U_{1 \text { conv }}$ are obtained from total pressure measurements along the CVP vortex path $s_{v}$. A Venturi Kiel probe was used to measure the total pressures.

The $X$ and $Z$ positioning of the probe was determined from the flow visualizations of figure 3.4. The trajectory of $s_{v}$ was assumed to lie half way in between the outlines of the smoke-tagged jet. The spanwise $(Y)$ location of a CVP vortex center was determined from the experimental results of Rajaratnam \& Gangadharaiah (1983); see their figure 4. The Kiel probe was aligned to be locally tangent to $s_{v}$.

The quantity measured was the difference between the crossflow total pressure $\left(p_{t c f}\right)$ and the CVP vortex total pressure $\left(p_{t s_{v}}\right)$ :

$$
\Delta p_{t}=p_{t s_{v}}-p_{t c f}
$$


The total pressures can be expressed as

$$
p_{t s_{v}}=p_{s_{v}}+\frac{1}{2} \rho U_{s_{v}}^{2}, \quad p_{t c f}=p_{c f}+\frac{1}{2} \rho U_{c f}^{2}
$$

Here, the velocities $U_{s_{v}}$ and $U_{c f}$ are the velocities in the directions locally tangent to $s_{v}$ and the crossflow, respectively. It is assumed that the other velocity components are negligible. The pressures and velocities in equation B.8 can be expressed in terms of their mean and fluctuating parts:

$$
p_{s_{v}}=\bar{p}_{s_{v}}+p_{s_{v}}^{\prime}, U_{s_{v}}=\bar{U}_{s_{v}}+u_{s_{v}}^{\prime}, p_{c f}=\bar{p}_{c f}+p_{c f}^{\prime}, U_{c f}=\bar{U}_{c f}+u_{c f}^{\prime}
$$

Substituting the equations of B.9 into the equations of B.8 and then into equation B.7 yields the following expression for the mean $\Delta p_{t}$ :

$$
\overline{\Delta p}_{t} \approx \frac{1}{2} \rho\left(\bar{U}_{s_{v}}^{2}-\bar{U}_{c f}^{2}\right)^{\frac{1}{2}}
$$

It is assumed, for the present purposes, that $\bar{p}_{s_{v}} \approx \bar{p}_{c f}, \overline{u_{c f}^{\prime}} \ll \bar{u}_{c f}^{2}$, and $\overline{u_{s_{v}}^{\prime}} \ll \bar{u}_{s_{v}}^{2}$. It follows from equation B.10 that an estimate for $U_{1 \text { conv }}$ is given by

$$
U_{1 \text { conv }}=\bar{U}_{s_{v}} \approx\left(\frac{2 \overline{\Delta p_{t}}}{\rho}+\bar{U}_{c f}^{2}\right)^{\frac{1}{2}}
$$




\section{References}

ANDREOPOULOS, J. 1985 On the structure of jets in a crossflow. J. Fluid Mech. 157, 163-197.

BAKER, C.J. 1980 The turbulent horseshoe vortex. J. Wind Engr. \& Ind. Aero. 6, 9-23.

BECKER, H.A. \& MASSARO, T.A. 1968 Vortex evolution in a round jet. J. Fluid Mech. 31, 435-448.

BOSANQUET, C.H. \& PEARSON, J.L. 1936 The spread of smoke and gases from chimneys. Transactions of the Faraday Society 32, 1249-1264.

BROADWELL, J.E. \& BREIDENTHAL, R.E. 1984 Structure and mixing of a transverse jet in incompressible flow. J. Fluid Mech. 148, 405-412.

BROWN, G.L. \& ROSHKO, A. 1971 The effect of density differences on the turbulent mixing layer. Turbulent Shear Flows. AGARD Conf. Proc. 93, 23.123.11 .

BROWN, G.L. \& ROSHKO, A. 1974 On density effects and large structure in turbulent mixing layers. J. Fluid Mech. 64, 775-816.

CHAISSANG, P., GEORGE, J., ClARIA, A. \& SANANES, F. 1974 Physical characteristics of subsonic jets in a cross stream. J. Fluid Mech. 62, 41-64.

CIMBALA, J.M. 1984 Large structure in the far wakes of two-dimensional bluff bodies. Ph.D. thesis, California Institute of Technology.

COEHLO, S.L.V. \& HUNT, J.C.R. 1989 The dynamics of the near field of strong jets in crossflows. J. Fluid Mech. 200, 95-120. 
COLES, D.C. 1985 Private communication. California Institute of Technology.

CORKE, T., KOGA, D., DRUBKA, R. \& NAGIB, H. 1977 A new technique for introducing controlled sheets of streaklines in wind tunnels. IEEE publication 77CH1251-8 AES.

CROW, S.C. \& CHAMPAGNE, F.H. 1971 Orderly structure in jet turbulence. $J$. Fluid Mech. 48, 547-591.

DAHM, W.J.A. \& DIMOTAKIS, P.E. 1985 Measurements of entrainment and mixing in turbulent jets. AIAA Paper 85-0056.

DIMOTAKIS, P.E. 1985 Private communication. California Institute of Technology.

DIMOTAKIS, P.E. \& BROWN, G.L. 1976 The mixing layer at high Reynolds number: large-structure dynamics and entrainment. J. Fluid Mech. 78, 535-560.

DIMOTAKIS, P.E., MIAKE-LYE, R.C. \& PAPANTONIOU, D.A. 1983 Structure and dynamics of round turbulent jets. Phys. Fluids 26, 3185-3192.

DOBSON, G.M.B. 1919 Measurements of turbulence in the atmosphere by the spreading of a smoke trail. RAE RM No. 671.

DURANDO, N.A. 1971 Vortices induced in a jet by a subsonic crossflow. AIAA J. 9, 325-327.

FEARN, R. \& WESTON, R.P. 1974 Vorticity associated with a jet in a cross flow. AIAA J. 12, 1666-1671.

FEARN, R. \& WESTON, R.P. 1975 Induced pressure distribution of a jet in a crossflow. NASA TN D-7916.

FOSS, J.F. 1980 Interaction region phenomena for the jet in a cross-flow problem. Rep. SFB 80/E/161, University of Karlsruhe. 
FREYMUTH, P. 1966 On transition in a separated laminar boundary layer. J. Fluid Mech. 25, 683-704.

GUTMARK, E. \& HO, C.M. 1983 Preferred modes and the spreading rates of jets. Phys. Fluids 26, 2932-2938.

HORNUNG, H.G. 1988 Vorlesungen über Themen aus der Strömungsphysik in Fachbereich Physik der Georg-August-Universität Göttingen, Band 1: Wirbelsträrkequellen.

HORNUNG, H.G. 1990 Private communication. California Institute of Technology.

HORNUNG, H.G. \& PERRY, A.E. 1984 Some aspects of three dimensional separation, part I: streamsurface bifurcations. Z. Flugwiss. Weltraumforsch 8, 77-87.

HOTTEL, H.C. 1953 Burning in laminar and turbulent fuel jets. In Proc. 4th Symp. (Intl) on Combustion, 97.

HUNT, J.C.R., ABELL, C.J., PETERKA, J.A. \& WOO, H. 1978 Kinematical studies of the flows around free or surface-mounted obstacles; applying topology to flow visualization. J.Fluid Mech. 86, 179-200.

KAMOTANI, Y. \& GREBER, I. 1972 Experiments on a turbulent jet in a cross flow. AIAA J. 10, 1425-1429.

KAMOTANI, Y. \& GREBER, I. 1974 Experiments on confined turbulent jets in crossflow. NASA CR2392.

KARAGOZIAN, A.R. 1986 An analytical model for the vorticity associated with a tranverse jet. AIAA J. 24, 429-436.

KEFFER, J.F. \& BAINES, W.D. 1963 The round turbulent jet in a cross-wind. $J$. Fluid Mech. 15, 481-496. 
KIYA, M., OHYAMA, M. \& HUNT, J.C.R. 1986 Vortex pairs and rings interacting with shear layer vortices. J. Fluid Mech. 172, 1-15.

KRAUSCHE, D., FEARN, R.L. \& WESTON, R.P. 1978 Round jet in a cross flow: influence of injection angle on vortex properties. AIAA J. 16, 636-637.

KROTHAPALLI, A., LOURENCO, L. \& BUCHLIN, J.M. 1990 Separated flow upstream of a jet in a crossflow. AIAA J. 28, 414-420.

KUZO, D.M. \& ROSHKO, A. 1984 Observations on the wake region of the transverse jet. Bulletin of the American Physical Society 29, 1536.

LE GRIEVES', E. 1978 Mixing process induced by the vorticity associated with the penetration of a jet into a cross flow. J. Eng. Power 100, 465-475.

LEIPMANN, H.W. 1945 Investigation of boundary layer transition on concave walls. NACA Wartime Rep. W-87.

LIGHTHILL, M.J. 1963 in Laminar Boundary Layers, ed. by L. Rosenhead, Oxford Univ. Press.

LOEHRKE, R.I. \& NAGIB, H.M. 1976 Control of free-stream turbulence by means of honeycombs: a balance between suppression and generation. Trans. ASME I: J. Fluids Engr. 98, 342-352.

MARGASON, M.J. \& FEARN, R. 1969 Jet-wake characteristics and their induced aerodynamic effects on V/STOL aircraft in transition flight. NASA SP218.

MASON, P.J. \& MORTON, B.R. 1987 Trailing vortices in the wakes of surfacemounted obstacles. J. Fluid Mech. 175, 247-293.

MCALLISTER, J.D. 1968 A momentum theory for the effects of cross flow on incompressible jets. PhD. thesis, Univ. of Tennessee. 
MCMAHON, H.M., HESTER, D.D. \& PALFREY, J.G. 1971 Vortex shedding from a turbulent jet in a cross-wind. J. Fluid Mech. 48, 73-80.

MEHTA, R.D. \& BRADSHAW, P. 1979 Design rules for small low speed wind tunnels. Aero. J. 83, 443-449.

MOREL, T. 1975 Comprehensive design of axisymmetric wind tunnel contractions. Trans. ASME I: J. Fluids Engr. 97, 225-233.

MORTON, B.R. 1984 The generation and decay of vorticity. Geophys. Astrophys. Fluid Dynamics 28, 277-308.

MOUSSA, Z.M., TRISCHKA, J.W. \& ESKANAZI, S. 1977 The near field in the mixing of a round jet with a cross-stream. J. Fluid Mech. 80, 49-80.

NAGIB, H.M., MARION, A. \& TAN-ATICHAT, J. 1984 On the design of contractions and settling chambers for optimal turbulence manipulation in wind tunnels. AIAA Paper 84-0536.

NUNN, R.H. 1985 Vorticity growth and decay in the jet in crossflow. AIAA J. 23, 473-475.

OKAMOTO, T. \& YAGITA, M. 1973 The experimental investigation on the flow past a cylinder of finite length placed normal to the plane surface in a uniform stream. Bull. JSME 16, 805-814.

PERRY, A.E. \& HORNUNG, H.G. 1984 Some aspects of three-dimensional separation, part II: vortex skeletons. Z. Flugwiss. Weltraumforsch 8, 77-87.

PRATTE, B.D. \& BAINES, M. 1967 Profiles of the round turbulent jet in a crossflow. J. Hydronaut. Div. ASCE 92, 53-64.

RAJARATNAM, N. \& GANGADHARAIAH, T. 1983 Vortex structure of circular jets in crossflow. J.Wind Eng. and Industrial Aero. 12, 155-164. 
REILLY, R.S. 1968 Investigation of the deformation and penetration of a turbulent, subsonic jet issuing transversely into a uniform, subsonic main stream. $\mathrm{PhD}$. thesis, Univ. of Maryland.

ROSHKO, A. 1953 On the development of turbulent wakes from vortex streets. NACA TN 2913.

ROSHKO, A. 1976 Structure of turbulent shear flows; a new look. AIAA J. 14, 1349-1357.

SCORER, R.S. 1958 Natural Aerodynamics Pergamon Press.

SYKES, R.I., LEWELLEN, W.S. \& PARKER, S.F. 1986 On the vorticity dynamics of a turbulent jet in a crossflow. J. Fluid Mech. 80, 49-80.

THOMAS, A.S.W. 1986 The unsteady characteristics of laminar junction flow. Phys. Fluids 30, 283-285.

THOMPSON, A.M. 1972 Comment on "vortices induced in a jet by a subsonic cross flow". AIAA J. 10, 364-365.

THWAITES, B. 1949 Approximate calculation of the laminar boundary layer. Aero. Quarterly 1, 245-280.

TSO, J., KOVASNAY, L.S.G. \& HUSSAIN, A.K.M.F. 1981 Search for large scale structures in the nearly self-preserving region of a turbulent axisymmetric jet. Trans. ASME I: J. Fluids Engr. 103, 503-508.

WINANT, C.D. \& BROWAND, F.K. 1974 Vortex pairing: the mechanism of turbulent mixing layer growth at moderate Reynolds numbers. J. Fluid Mech. 63, 237.

WU, J.Z., GU, J.W. \& WU, J.M. 1987 Steady three-dimensional fluid particle separation from arbitrary smooth surface and formation of free vortex layers. 5th AIAA Appl. Aerodyn. Conf., Monterey. 
WU, J.M., VAKILI, A.D. \& YU, F.M. 1988 Investigation of the interacting flow of nonsymmetric jets in crossflow. AIAA J. 26, 940-947.

WU, J.Z., WU, J.M. \& WU, C.J. 1987 A general 3-dimensional viscous compressible theory on the interaction of solid body and vorticity-dilatation field. UTSI Report 87/03.

YULE, A.J. 1978 Large-scale structure in the mixing layer of a round jet. J. Fluid Mech. 89, 413-432. 


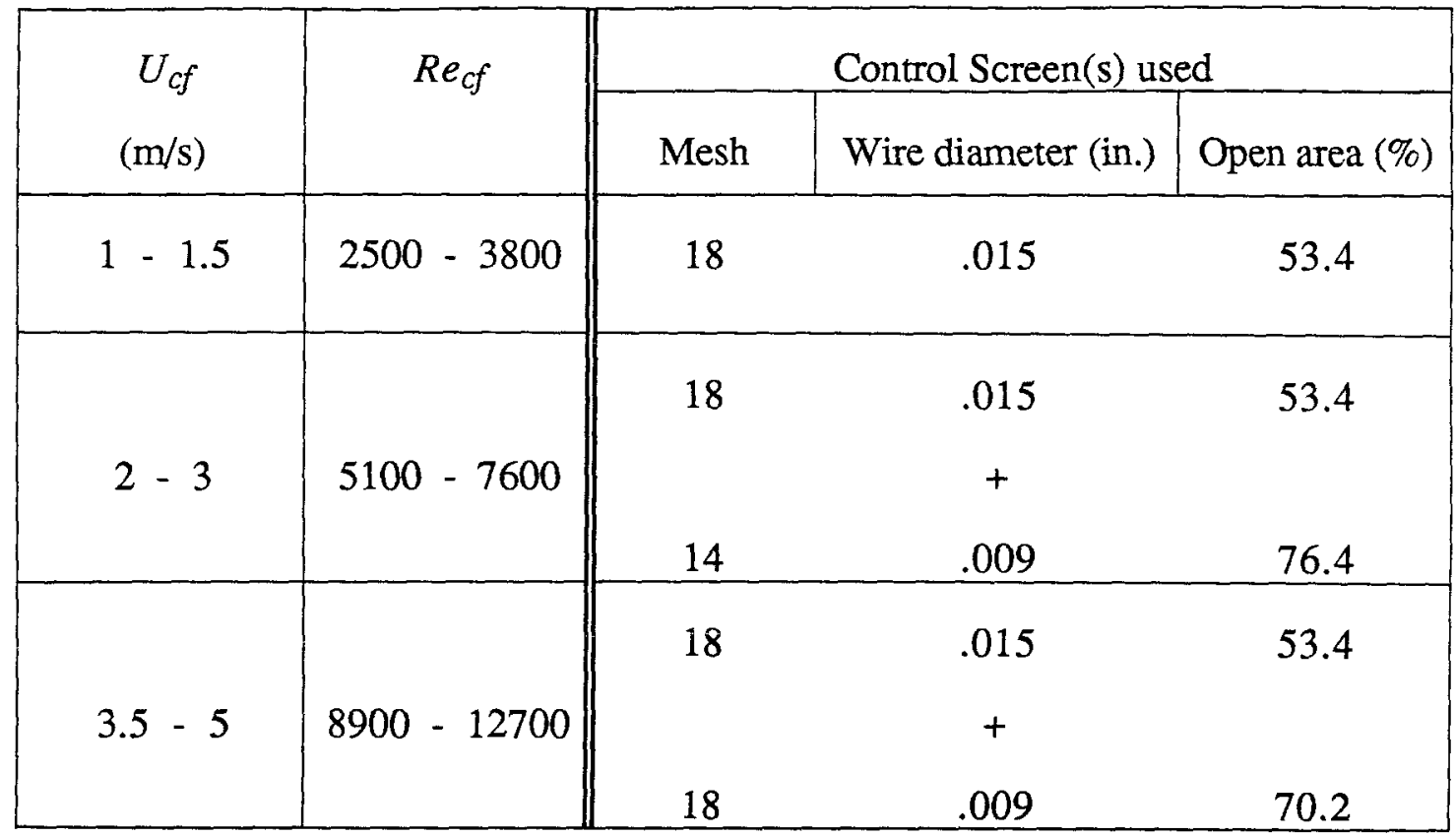

TABLE 2.1: Control screen(s) used at the end of the wind tunnel test section.

\begin{tabular}{|c|c|c||cc|cc|c|}
\hline$U_{c f}$ & $R e_{c f}$ & $L_{j} / D_{j}$ & $\delta_{c f} D_{j}$ & $\delta_{c f} / D_{j}$ & $\theta_{c f} / D_{j}$ & $\theta_{c f} / D_{j}$ & $u_{r m s}^{\prime} / U_{c f}$ \\
$(\mathrm{~m} / \mathrm{s})$ & & $($ measured) & (Blasius) & (measured) & (Blasius) & (where $\left.U / U_{c f}=.5\right)$ \\
\hline 1.5 & 3800 & 5 & $\begin{array}{c}.056 \\
.087\end{array}$ & .062 & .026 & .024 & .0046 \\
& & 10 & .088 & .037 & .034 & .0046 \\
\hline 3.0 & 7600 & 5 & .039 & .044 & .020 & .017 & .0044 \\
& & 10 & .055 & .062 & .024 & .024 & .0041 \\
\hline 4.5 & 11400 & 5 & .029 & .036 & .014 & .014 & .0073 \\
& & 10 & -- & .051 & - & .020 & .0040 \\
\hline
\end{tabular}

TABLE 2.2: Characteristics of the crossflow boundary layer at $X / D_{j}=0$. Nominal values are shown, i.e., no jet is present. 


\begin{tabular}{|c|c||c|c|c|}
\hline$U_{j}$ & $R e_{j}$ & $\delta_{j} / D_{j}$ & $\theta_{j} / D_{j}$ & $w_{r m s}^{\prime} / U_{j}$ \\
$(\mathrm{~m} / \mathrm{s})$ & & $\begin{array}{c}\text { (measured) } \\
\text { (measured) }\end{array}$ & $(X=Y=0)$ \\
\hline 3 & 7600 & .045 & .0093 & .012 \\
6 & 15200 & .039 & .0067 & .012 \\
9 & 22900 & .037 & .0056 & .012 \\
12 & 30500 & .035 & .0048 & .012 \\
15 & 38100 & .033 & .0043 & .010 \\
18 & 45700 & .032 & .0035 & .0098 \\
24 & 61000 & .031 & .0031 & .0079 \\
27 & 68600 & .031 & .0030 & .0072 \\
30 & 76200 & .031 & .0028 & .0067 \\
36 & 91400 & .030 & .0026 & .0062 \\
45 & 114000 & .029 & .0024 & .0052 \\
\hline
\end{tabular}

TABLE 2.3: Jet characteristics at $Z / D_{j}=0$. All values shown are nominal, i.e., there is no crossflow. Jet is issuing into a closed wind tunnel. 


\begin{tabular}{|c|c|c|}
\hline$R e_{c f}$ & $V R$ or $V R_{\text {nominal }}$ & $V R_{\text {actual }}$ \\
\hline \multirow[t]{5}{*}{3800} & 2 & 2.26 \\
\hline & 4 & 4.19 \\
\hline & 6 & 6.17 \\
\hline & 8 & 8.16 \\
\hline & 10 & 10.5 \\
\hline \multirow[t]{5}{*}{7600} & 2 & 2.07 \\
\hline & 4 & 3.98 \\
\hline & 6 & 6.12 \\
\hline & 8 & 8.3 \\
\hline & 10 & 10.6 \\
\hline \multirow[t]{5}{*}{11400} & 2 & 2.02 \\
\hline & 4 & 4.08 \\
\hline & 6 & 6.23 \\
\hline & 8 & 8.42 \\
\hline & 10 & 10.7 \\
\hline
\end{tabular}

TABLE 2.4: Comparison of nominal and actual jet to crossflow velocity ratios. ( $U_{c f}$ for actual values was measured at $X / D_{j}=-15$, not 0 .) 


\begin{tabular}{|c|c|c||cc|cc|}
\hline$V R$ & $Z I D_{j}$ & $X I D_{j}$ & $\delta_{w} / D_{j}$ & $\delta_{w} / D_{j}$ & $\theta_{w} / D_{j}$ & $\theta_{w} / D_{j}$ \\
& & & $L_{j} / D_{j}=5$ & $L_{j} / D_{j}=10$ & $L_{j} / D_{j}=5$ & $L_{j} / D_{j}=10$ \\
\hline 4 & .5 & 1.5 & -.36 & -.36 & -.42 & -.45 \\
& .5 & 3.5 & $.25 *$ & $.29 *$ & $.23 *$ & $.24 *$ \\
& .5 & 5.5 & $.24 *$ & $.35 *$ & $.23 *$ & $.31 *$ \\
& .5 & 7.5 & $.19 *$ & $.38 *$ & $.18 *$ & $.34 *$ \\
\hline & 2.5 & 1.5 & .33 & .53 & -.20 & -.028 \\
& 2.5 & 3.5 & $.67 *$ & $.67 *$ & $.43 *$ & $.35 *$ \\
& 2.5 & 5.5 & $.36 *$ & $.35 *$ & $.30 *$ & $.27 *$ \\
& 2.5 & 7.5 & $.32 *$ & $.32 *$ & $.29 *$ & $.28 *$ \\
\hline & 4.5 & 3.5 & -.63 & -.26 & -.76 & -.35 \\
& 4.5 & 5.5 & $.89 *$ & $1.20 *$ & $.52 *$ & $.65 *$ \\
& 4.5 & 7.5 & $.92 *$ & $1.19 *$ & $.66 *$ & $.76 *$ \\
\hline
\end{tabular}

TABLE 5.1a: Measured estimates for wake displacement and momentum thicknesses. $V R=4, R e_{c f}=3800$. * indicates wake-like profile. 


\begin{tabular}{|c|c|c||cc|cc|}
\hline$V R$ & $Z I D_{j}$ & $X / D_{j}$ & $\begin{array}{c}\delta_{w} / D_{j} \\
L_{j} / D_{j}=5\end{array}$ & $\begin{array}{c}\delta_{w} / D_{j} \\
L_{j} / D_{j}=10\end{array}$ & $\begin{array}{c}\theta_{w} / D_{j} \\
L_{j} / D_{j}=5\end{array}$ & $\begin{array}{c}\theta_{w} / D_{j} \\
L_{j} / D_{j}=10\end{array}$ \\
\hline 8 & .5 & 1.5 & .47 & .61 & .21 & .29 \\
& .5 & 3.5 & $.54 *$ & $.64 *$ & $.40 *$ & $.54 *$ \\
& .5 & 5.5 & $.60 *$ & $.89 *$ & $.49 *$ & $.67 *$ \\
& .5 & 7.5 & $.50 *$ & $.80 *$ & $.44 *$ & $.63 *$ \\
\hline & 2.5 & 1.5 & .022 & .043 & -.18 & -.28 \\
& 2.5 & 3.5 & $.63 *$ & $.64 *$ & $.52 *$ & $.51 *$ \\
& 2.5 & 5.5 & $.41 *$ & $.54 *$ & $.36 *$ & $.46 *$ \\
& 2.5 & 7.5 & $.30 *$ & $.63 *$ & $.27 *$ & $.38 *$ \\
\hline \multirow{5}{*}{.5 .5} & 3.5 & .44 & .84 & .20 & .43 \\
& 4.5 & 5.5 & $.70 *$ & $.81 *$ & $.57 *$ & $.63 *$ \\
& 4.5 & 7.5 & $.46 *$ & $.63 *$ & $.42 *$ & $.54 *$ \\
\hline
\end{tabular}

TABLE 5.1b: Measured estimates for wake displacement and momentum thicknesses. $V R=8, R e_{c f}=3800$. * indicates wake-like profile. 


\begin{tabular}{|c|c|c||c|c|}
\hline & $Z / D_{j}$ & $X / D_{j}$ & $\begin{array}{c}\delta_{w} / D_{j} \\
L_{j} / D_{j}=5\end{array}$ & $\begin{array}{c}\theta_{w} / D_{j} \\
L_{j} / D_{j}=5\end{array}$ \\
\hline \multirow{2}{*}{ CYL. } & .5 & 1.5 & 1.1 & -.075 \\
& .5 & 3.5 & 1.2 & .41 \\
.5 & 5.5 & $1.4 *$ & $.67 *$ \\
.5 & 7.5 & $1.5 *$ & $.89 *$ \\
\cline { 2 - 4 } & 2.5 & 1.5 & 1.2 & -.009 \\
2.5 & 3.5 & 1.1 & .32 \\
& 2.5 & 5.5 & $.77^{*}$ & $.44^{*}$ \\
\hline
\end{tabular}

TABLE 5.1c: Measured estimates for wake displacement and momentum thicknesses. Cylinder of $A R=6, R e_{c f}=3800$.

* indicates wake-like profile.

\begin{tabular}{|c||cc|cc|}
\hline$V R$ & $\bar{\delta}_{w} / D_{j}$ & $\bar{\delta}_{w} / D_{j}$ & $\bar{\theta}_{w} / D_{j}$ & $\bar{\theta}_{w} / D_{j}$ \\
& $L_{j} / D_{j}=5$ & $L_{j} / D_{j}=10$ & $L_{j} / D_{j}=5$ & $L_{j} / D_{j}=10$ \\
\hline 4 & .48 & .59 & .35 & .40 \\
8 & .51 & .70 & .43 & .54 \\
CYLINDER & 1.1 & & .61 & \\
\hline
\end{tabular}

TABLE 5.2: Averages for wake displacement and momentum thicknesses using wake-like profiles from tables 5.1 only. $R e_{c f}=3800$. 


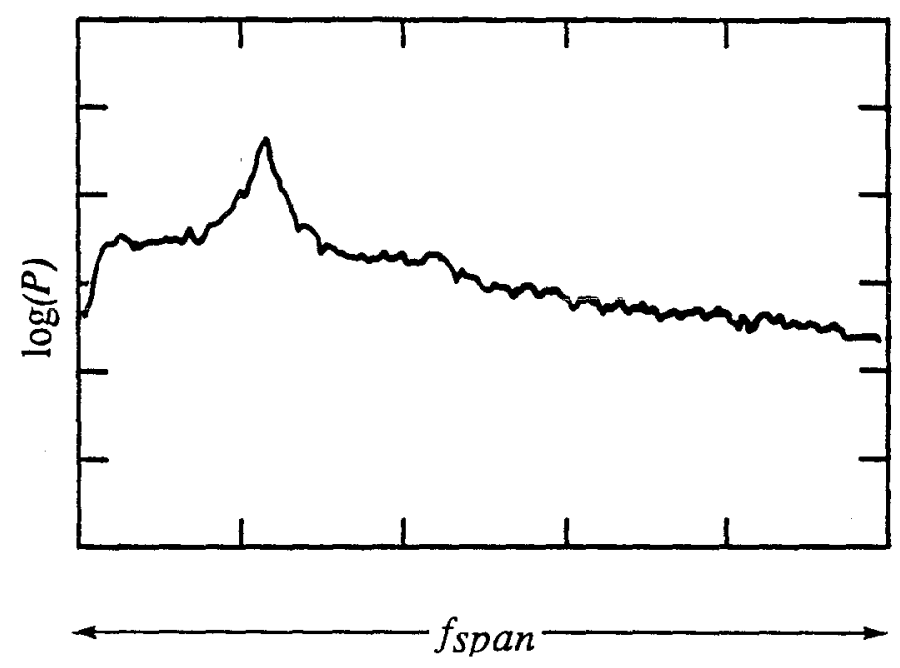

\begin{tabular}{|c|c|c|c|}
\hline Span & $\begin{array}{c}\text { Resolution } \\
f_{\text {Span }} \text { (Hz.) }\end{array}$ & $\begin{array}{c}\text { Sampling Rate } \\
\Delta f(\mathrm{~Hz} .)\end{array}$ & $\begin{array}{c}\text { Number of Samples } \\
\text { per Record }\end{array}$ \\
\hline 1 & .004 & 4 & 1024 \\
50 & .2 & 200 & 1024 \\
1000 & 4 & 4000 & 1024 \\
25000 & 100 & 100,000 & 1024 \\
\hline
\end{tabular}

TABLE B.1: HP 3582A Spectrum Analyzer sampling characteristics. 


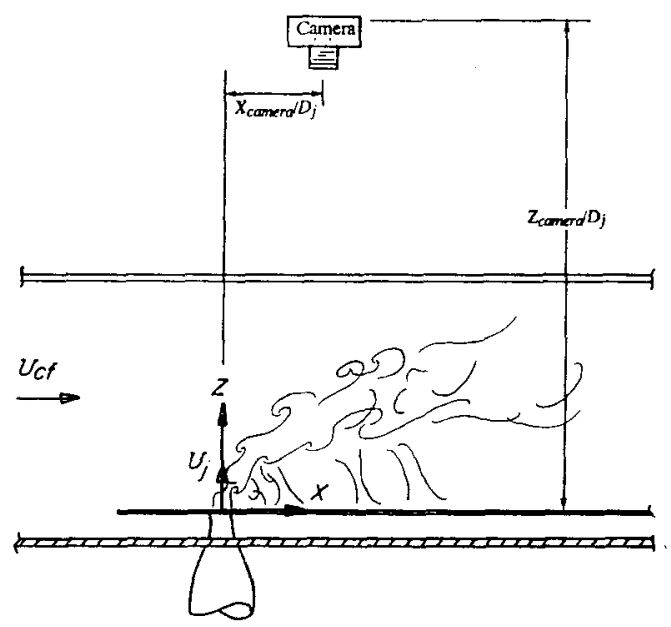

\begin{tabular}{|c||c|c|}
\hline Photograph & $X_{\text {camera }} D_{j}$ & $Z_{\text {cameral }} D_{j}$ \\
\hline $3.3 \mathrm{a}$ & 4 & 37 \\
$3.3 \mathrm{~b}$ & 1.5 & 20 \\
\hline $3.7 \mathrm{a}$ top & 0 & 20 \\
$3.7 \mathrm{a}$ center & 1.5 & 20 \\
$3.7 \mathrm{a}$ bottom & 4.5 & 37 \\
\hline $3.7 \mathrm{~b}$ top & 0 & 20 \\
$3.7 \mathrm{~b}$ center & 1.5 & 20 \\
$3.7 \mathrm{~b}$ bottom & 4.5 & 37 \\
\hline $3.7 \mathrm{c}$ top & 1 & 20 \\
$3.7 \mathrm{c}$ center & 4.5 & 37 \\
$3.7 \mathrm{c}$ bottom & 4.5 & 37 \\
\hline $3.7 \mathrm{~d}$ top & 4.5 & 37 \\
$3.7 \mathrm{~d}$ center & 4.5 & 37 \\
$3.7 \mathrm{~d}$ bottom & 4.5 & 37 \\
\hline $3.7 \mathrm{e}$ top & 4.5 & 37 \\
$3.7 \mathrm{e}$ center & 4.5 & 37 \\
$3.7 \mathrm{e}$ bottom & 4.5 & 37 \\
\hline $3.15 \mathrm{a}$ center & 3.5 & 37 \\
$3.15 \mathrm{a}$ bottom & 4.5 & 37 \\
\hline $3.15 \mathrm{~b}$ center & 4.5 & 37 \\
$3.15 \mathrm{~b}$ bottom & 4.5 & 37 \\
\hline $3.15 \mathrm{c}$ center & 4.5 & 37 \\
\hline $3.15 \mathrm{c}$ bottom & 4.5 & 37 \\
\hline $3.15 \mathrm{~d}$ center & 4.5 & 37 \\
\hline $3.15 \mathrm{~d}$ bottom & 4.5 & 37 \\
\hline $3.15 \mathrm{e}$ center & 4.5 & 37 \\
\hline $3.15 \mathrm{e}$ bottom & 4.5 & 37 \\
\hline $5.1 \mathrm{a}$ & 4.5 & 37 \\
\hline $5.1 \mathrm{~b}$ & 4.5 & 37 \\
\hline $5.20 \mathrm{~b}$ & 4.5 & 37 \\
\hline $5.20 \mathrm{c}$ & 4.5 & \\
\hline $5.21 \mathrm{a}$ & 4.5 & 37 \\
\hline $5.21 \mathrm{~b}$ & 4.5 & 37 \\
\hline & & 37 \\
\hline & & 37 \\
\hline
\end{tabular}

TABLE B.2: Camera location for photographs in which jet "issues at the viewer." 


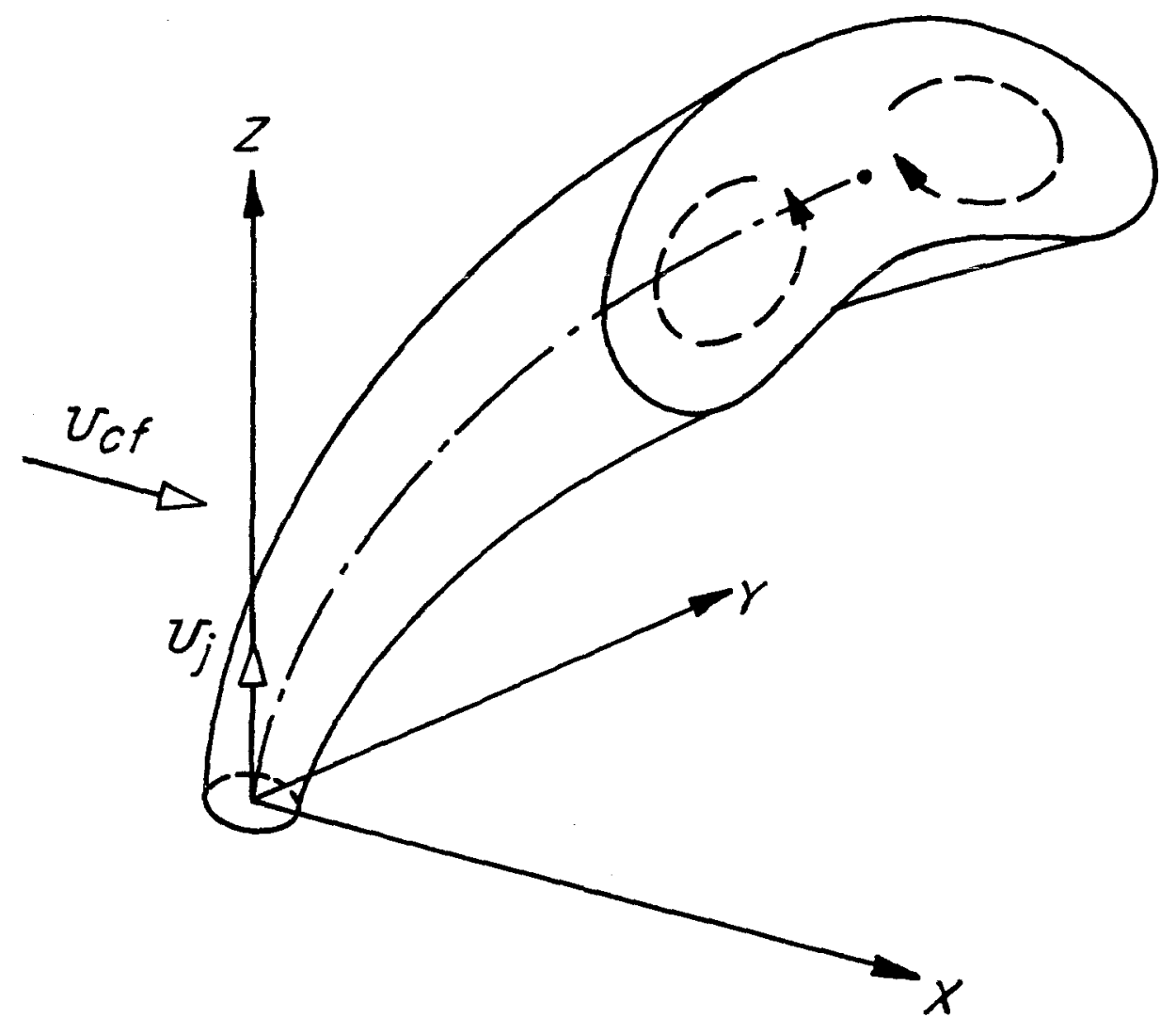

FIGURE 1.1: Counterrotating vortex pair structure of the transverse jet in the far field. 


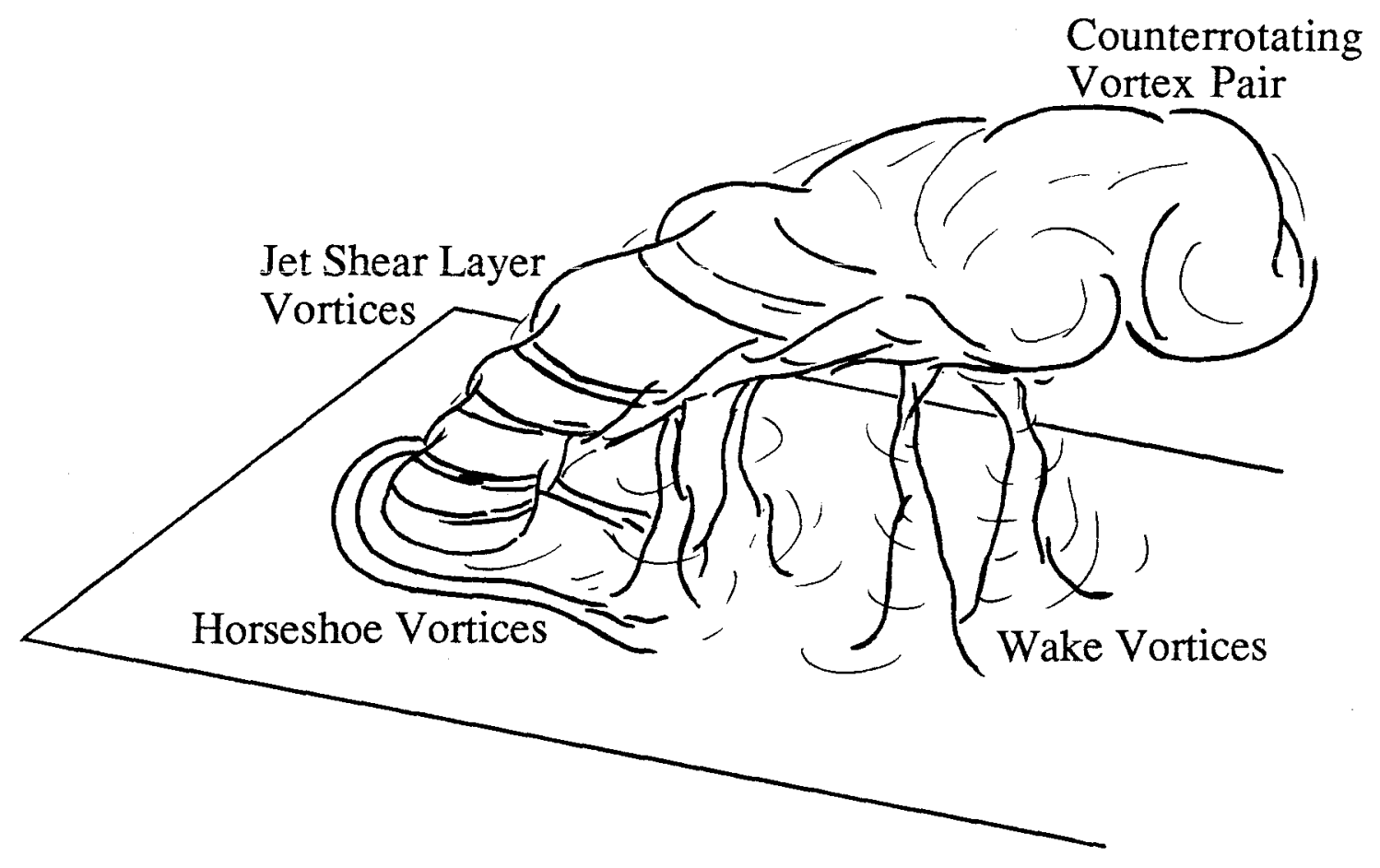

FIGURE 1.2: Structure in the near field of the transverse jet. 


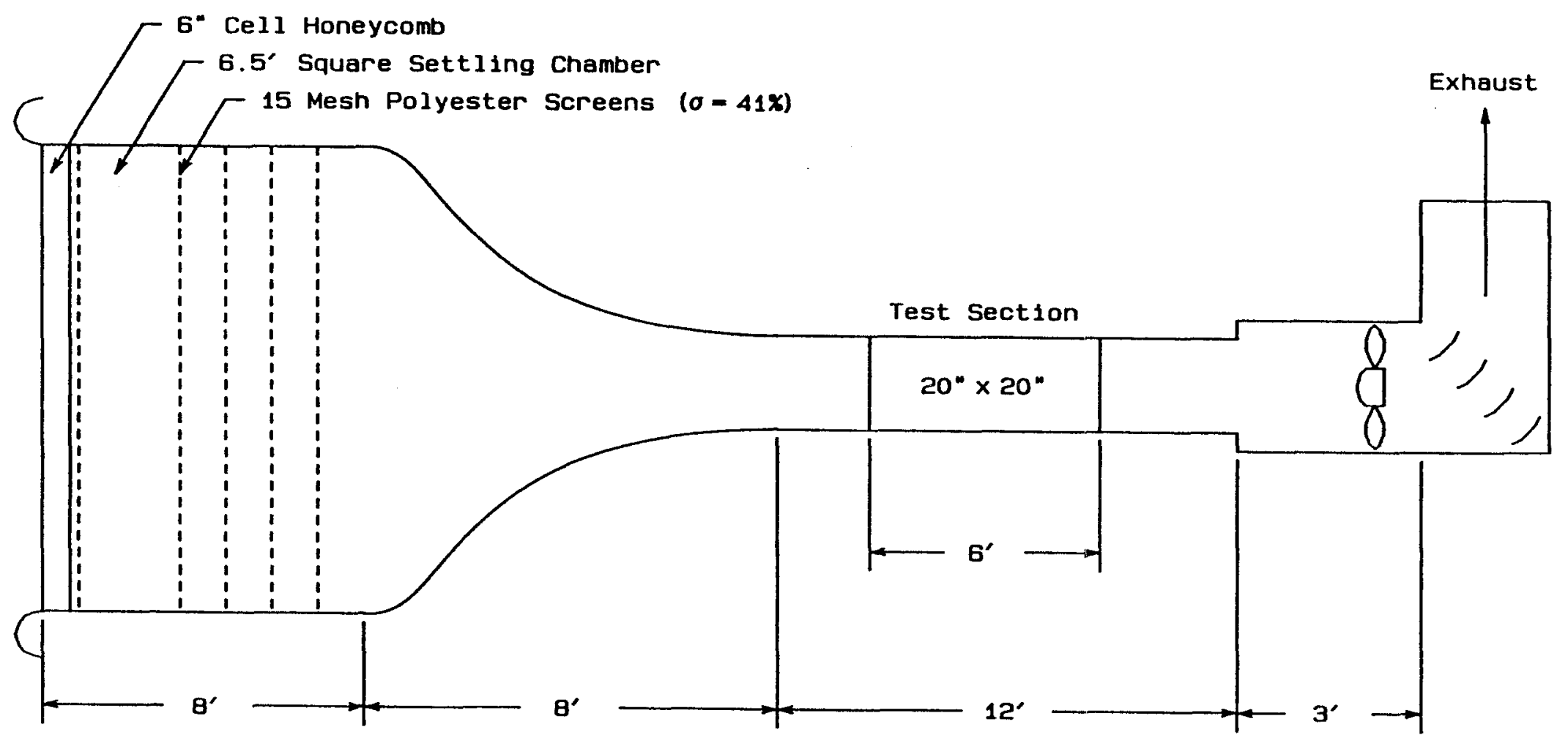

FIGURE 2.1: 20" X 20" open return wind tunnel.

From Cimbala (1984). (Not drawn to scale.) 


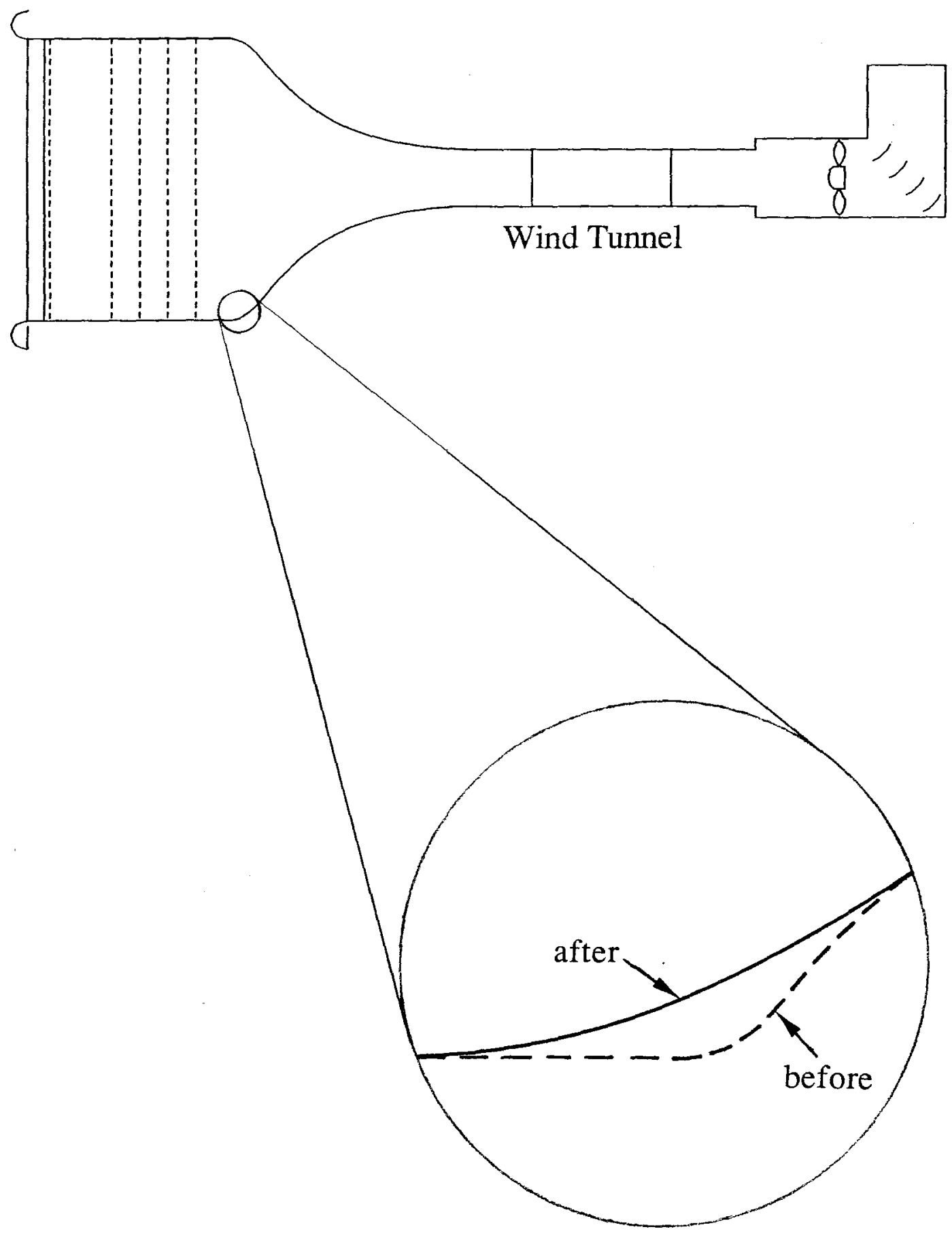

FIGURE 2.2: Wind tunnel contraction section modification. 


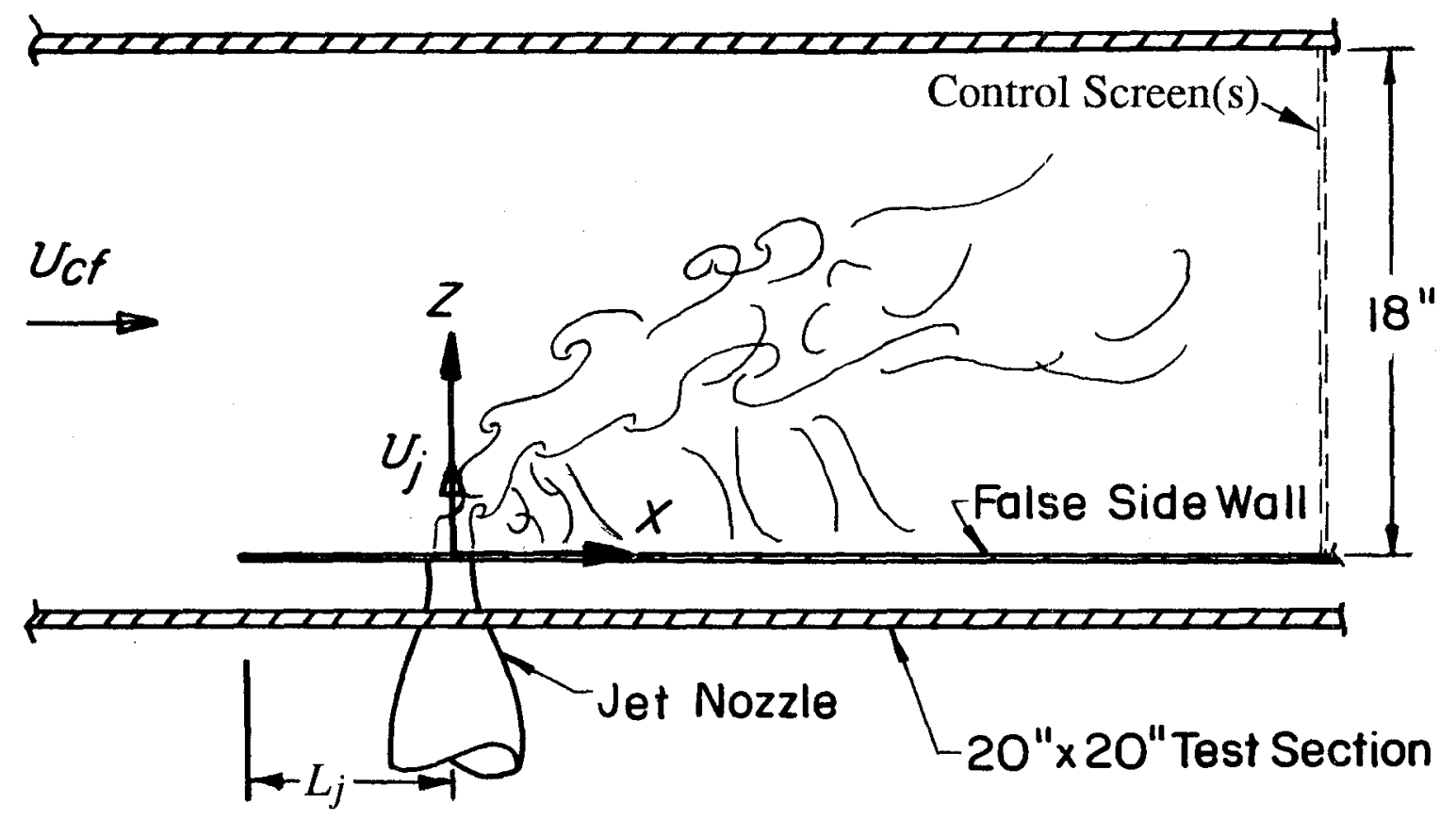

FIGURE 2.3: Detail of the test section with jet injected at $90^{\circ}$. 


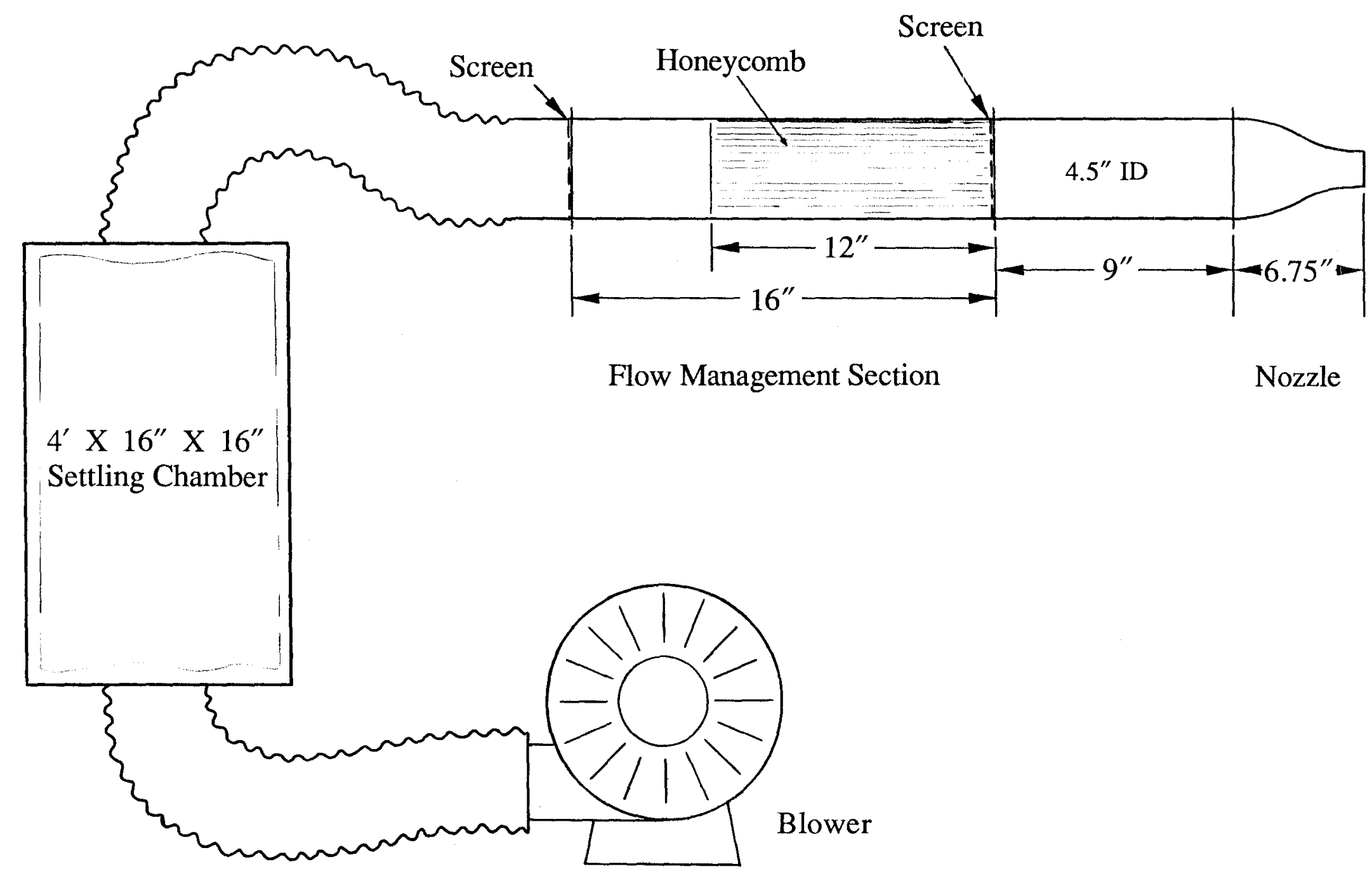

FIGURE 2.4: Schematic of the jet delivery system (not drawn to scale). 


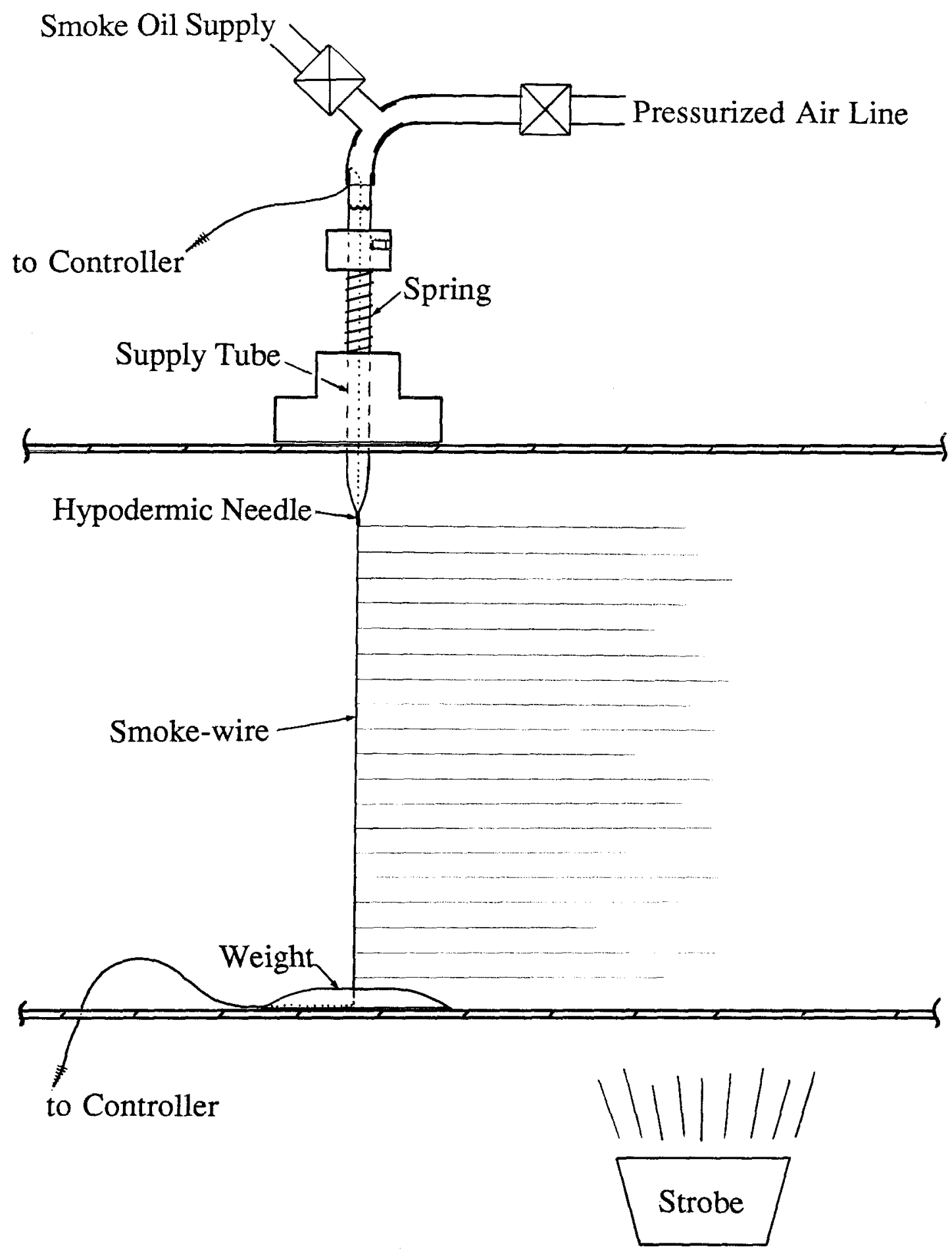

FIGURE 2.5: Smoke-wire traversing set-up (not drawn to scale). 

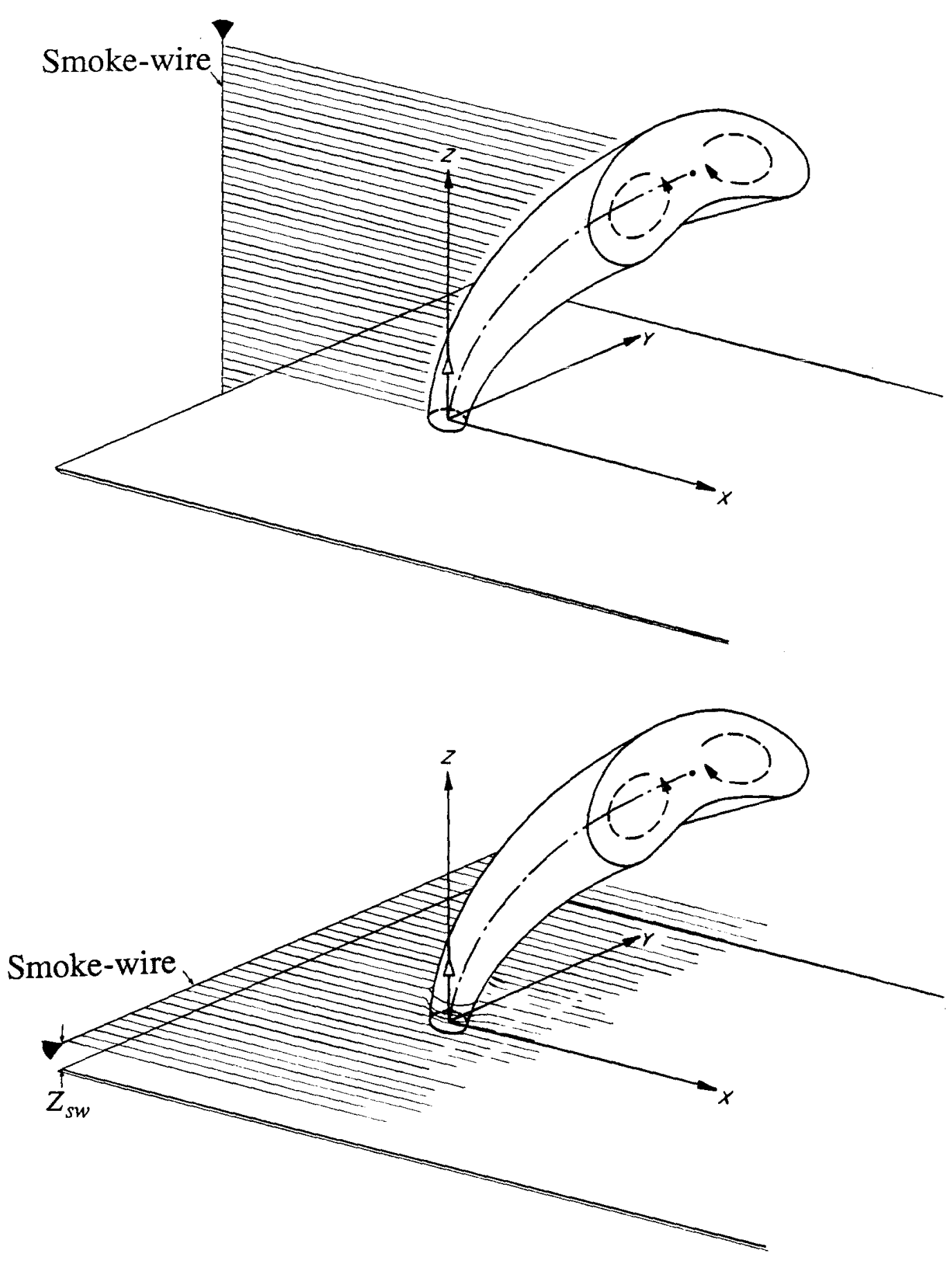

FIGURE 2.6: Typical smoke-wire/jet orientations. 


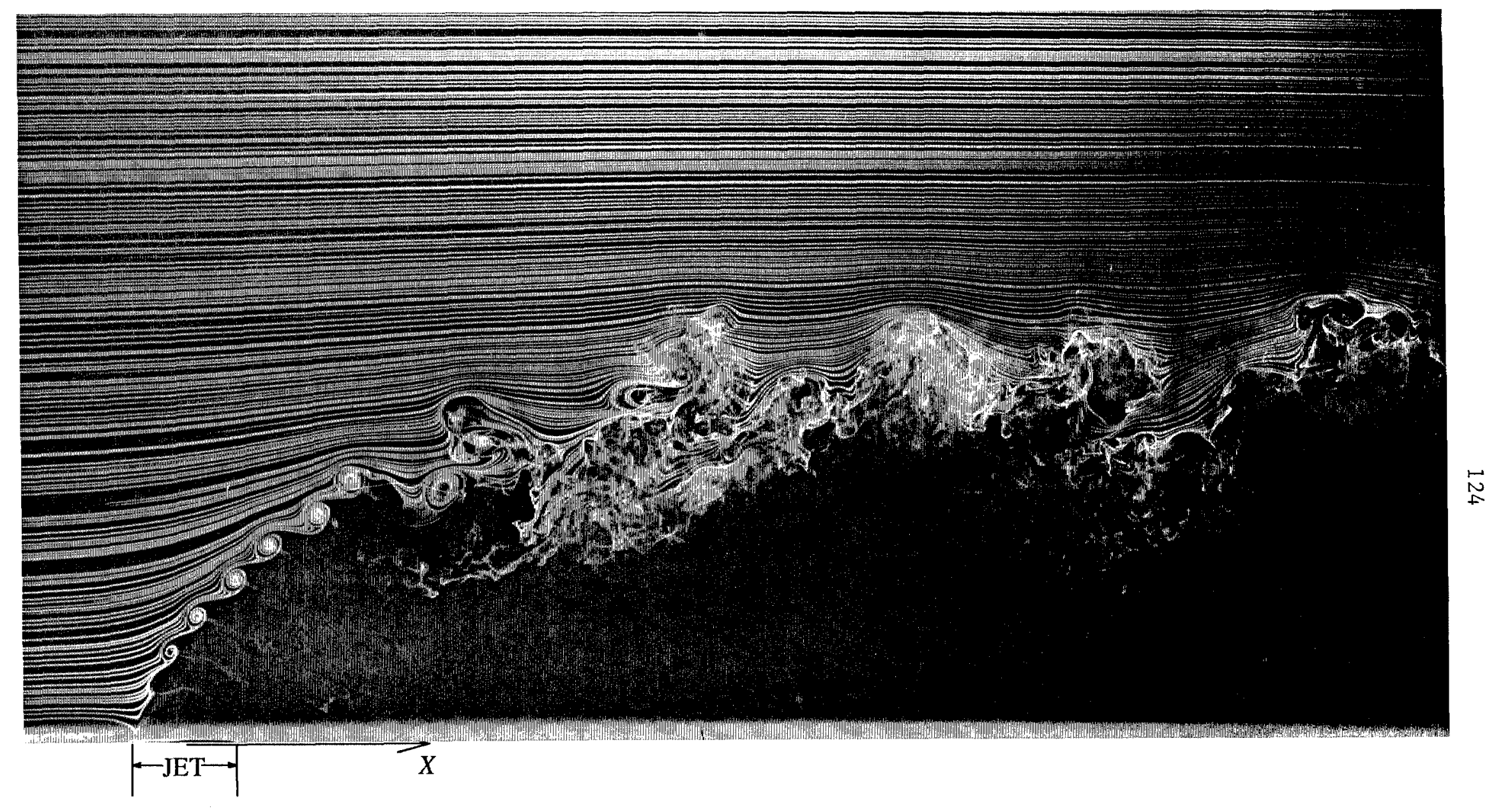

FIGURE 3.1a: $V R=2$.

FIGURE 3.1: Leading edge of the distorted shear layer structure of the deflected jet. (a) $V R=2$, (b) $V R=4$, (c) $V R=6$, (d) $V R=8$, (e) $V R=10$.

$R e_{c f}=3800 . Y_{s w} / D_{j}=0$ 

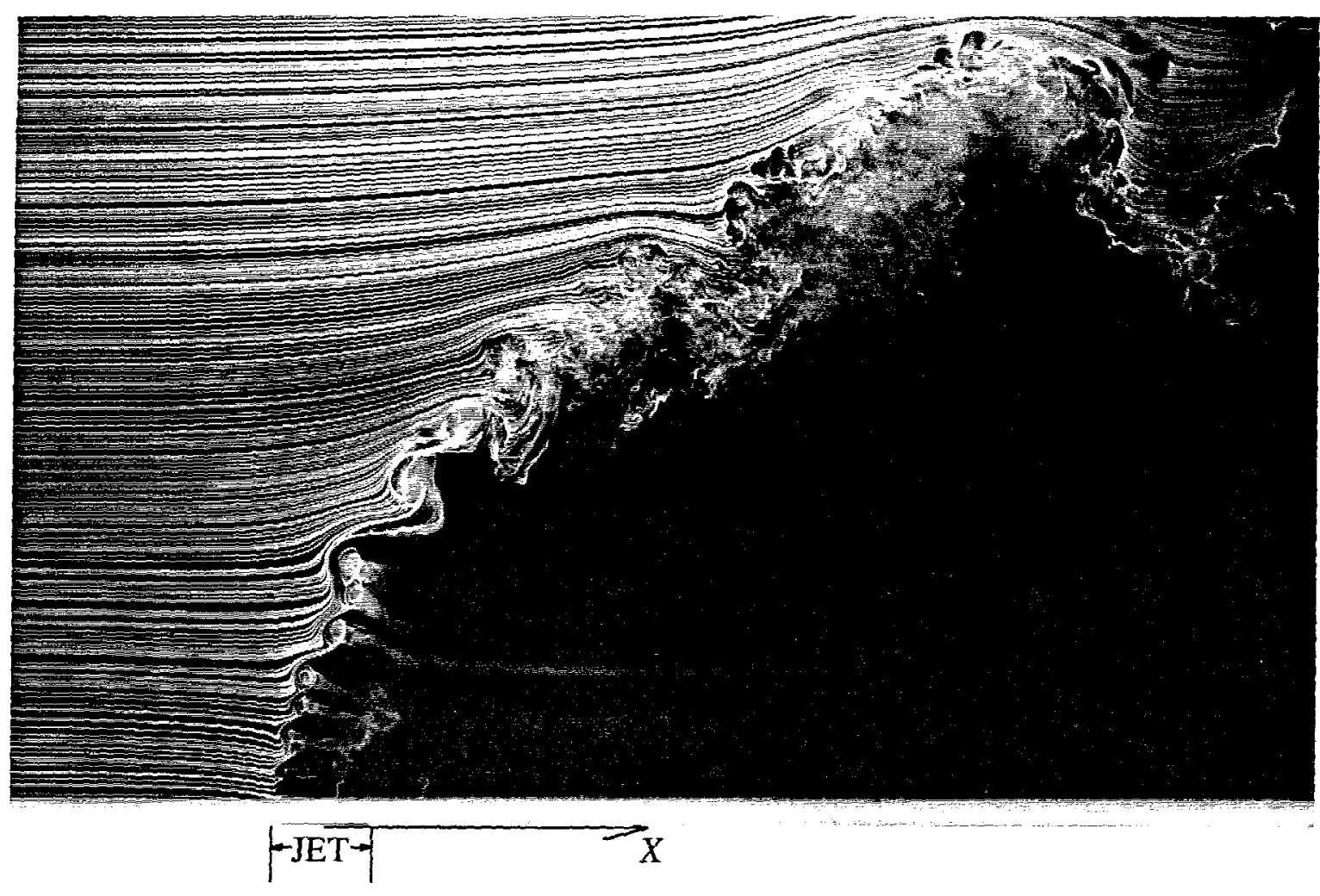

FIGURE 3.1b: $V R=4$.

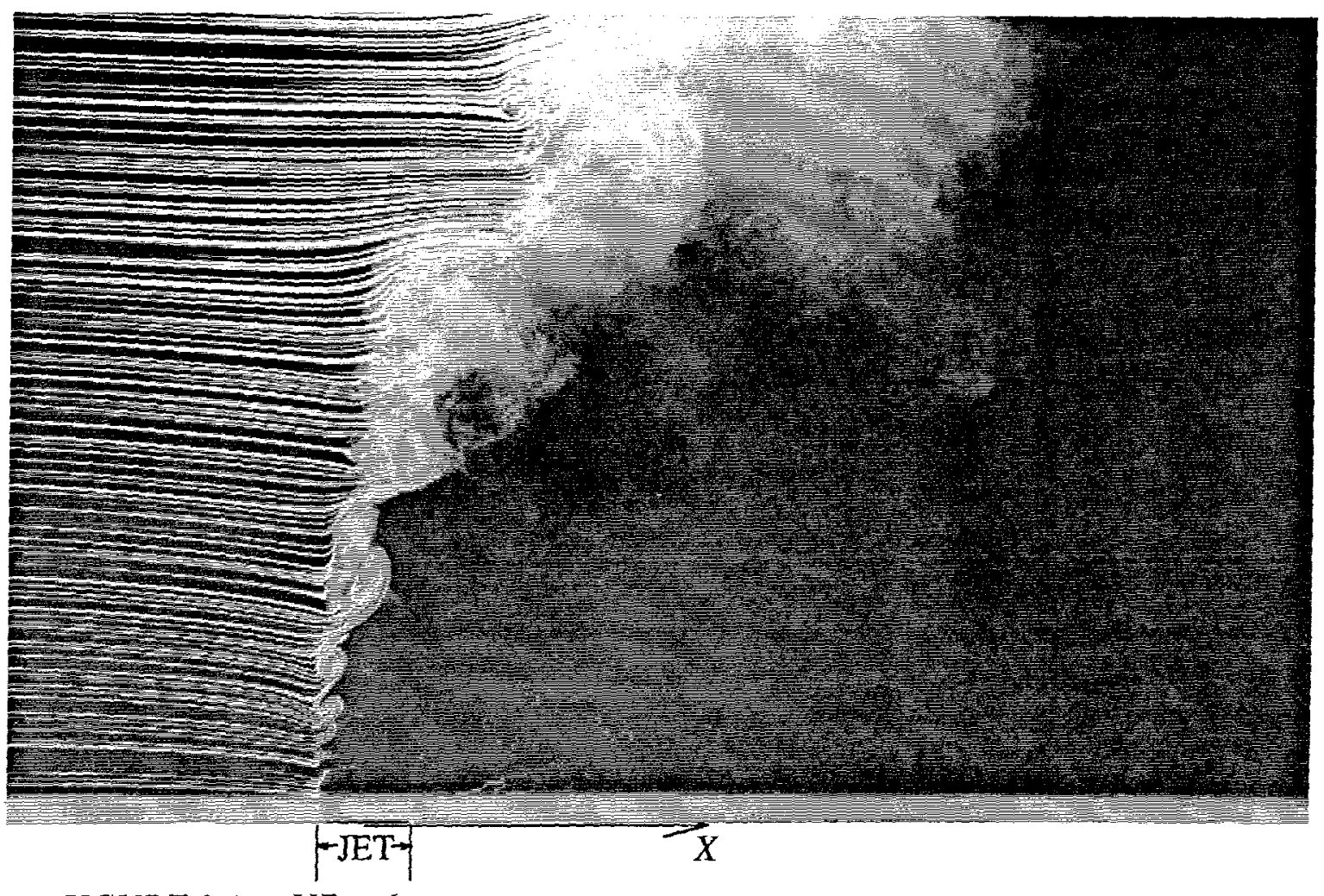

FIGURE 3.1c: $V R=6$. 

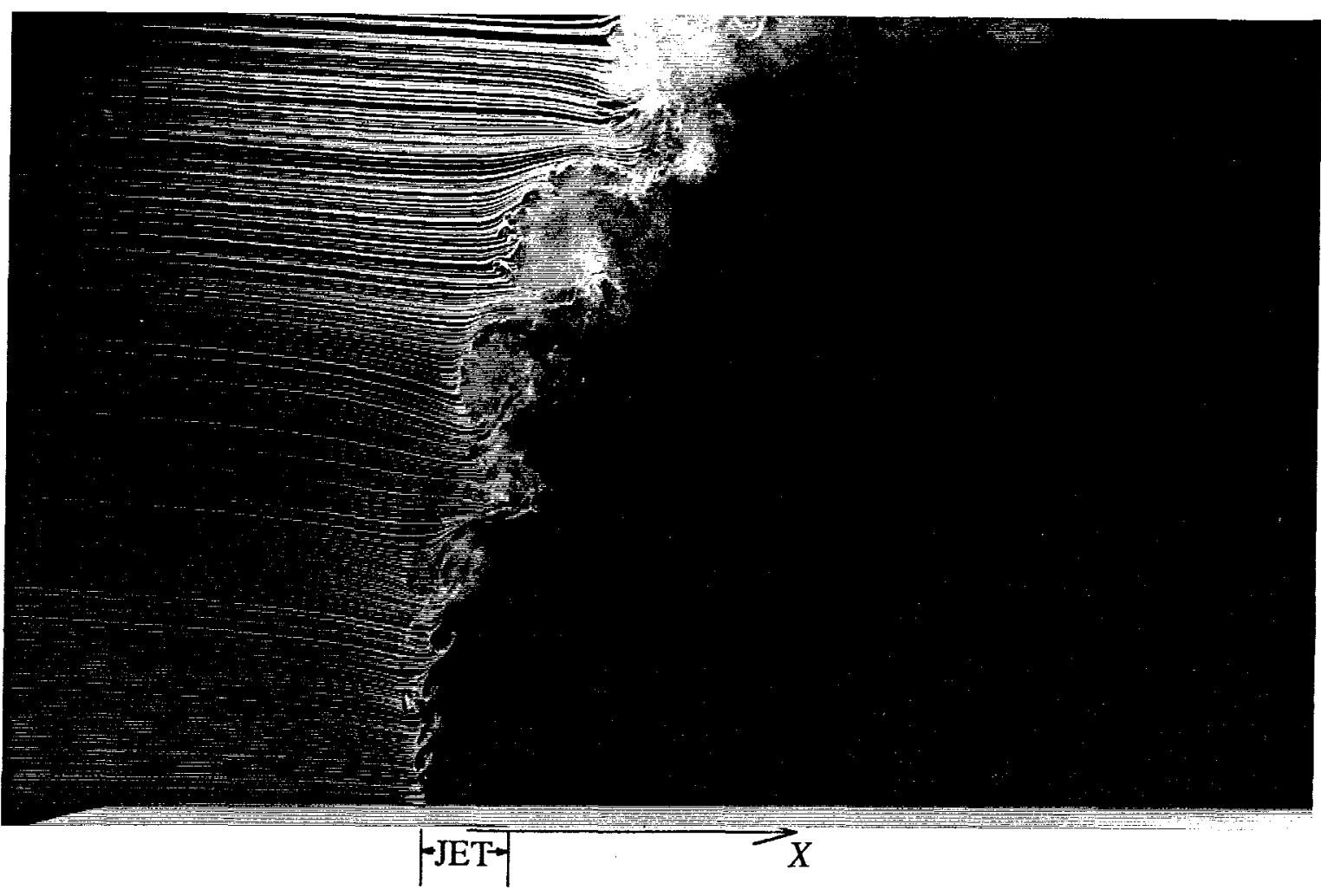

FIGURE 3.1d: $V R=8$.

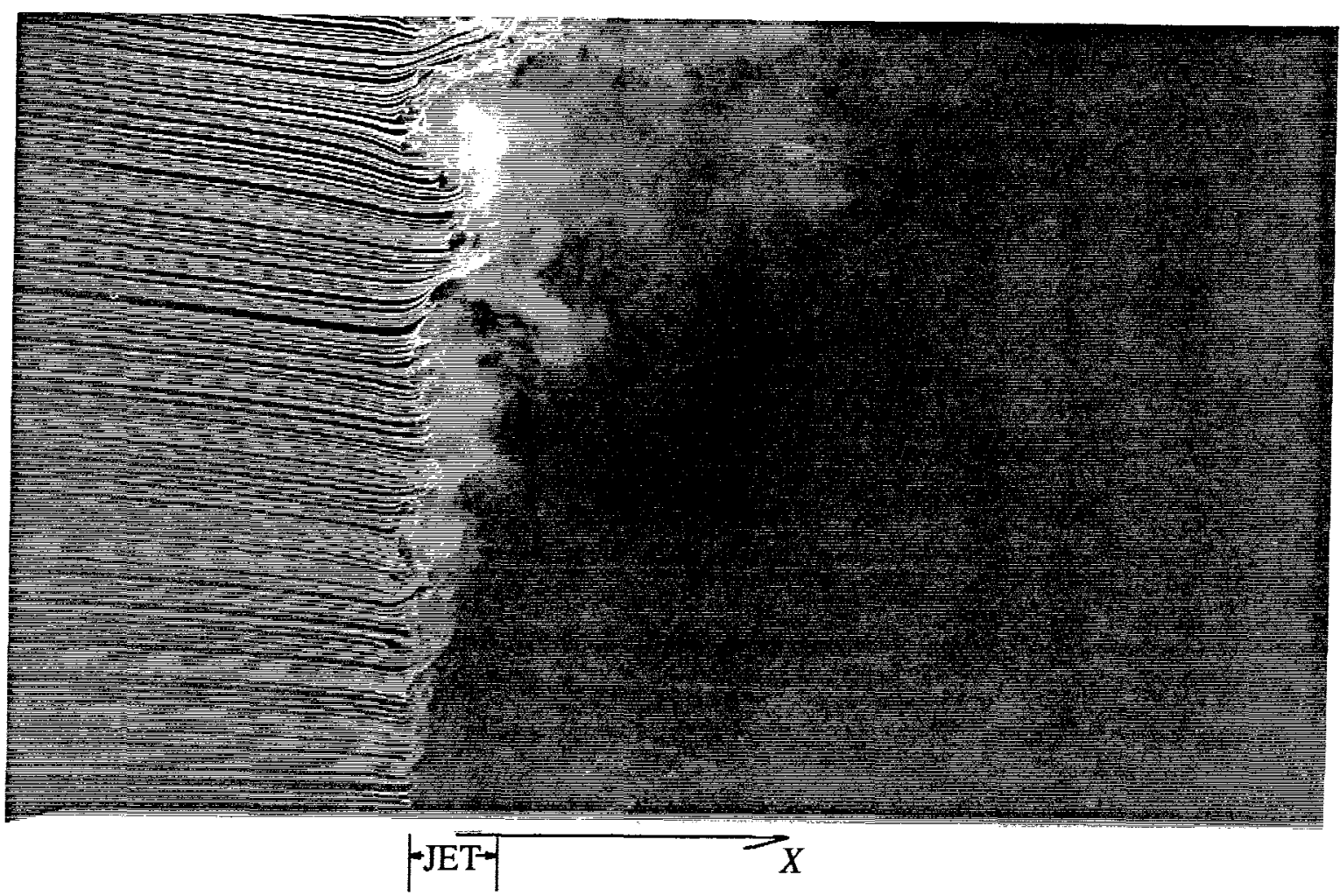

FIGURE 3.1e: $V R=10$. 


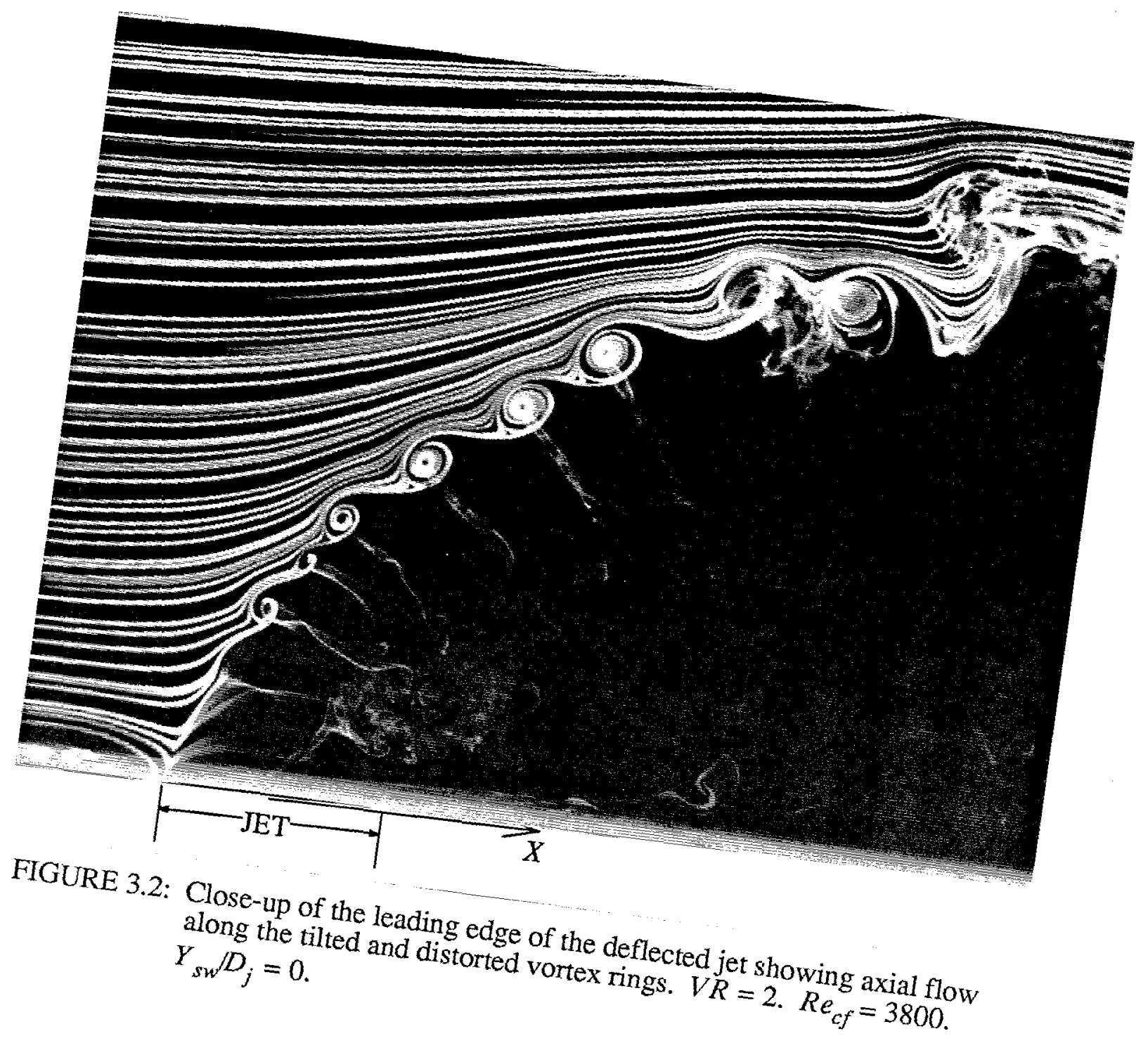




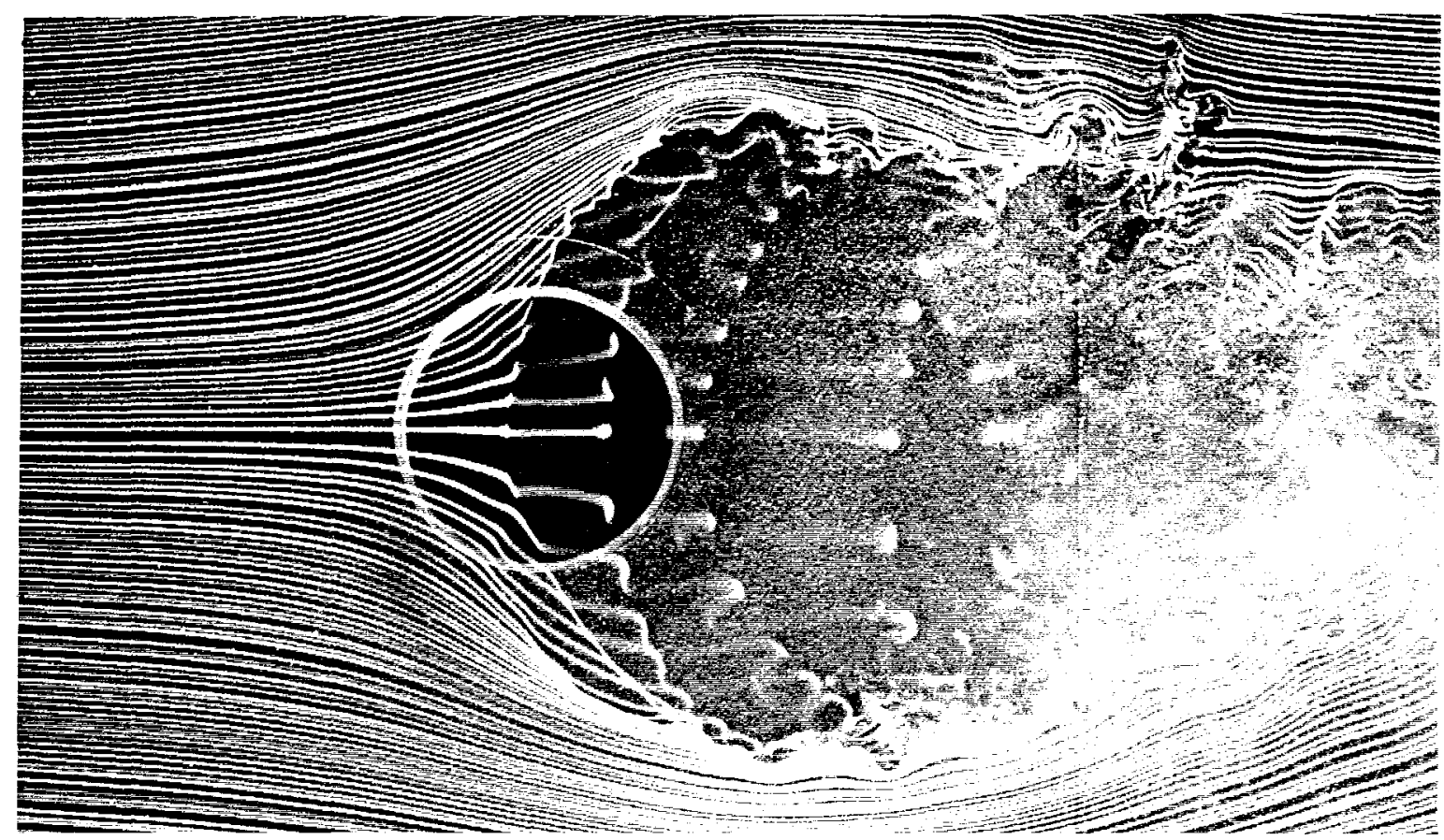

(a) $V R=2$.

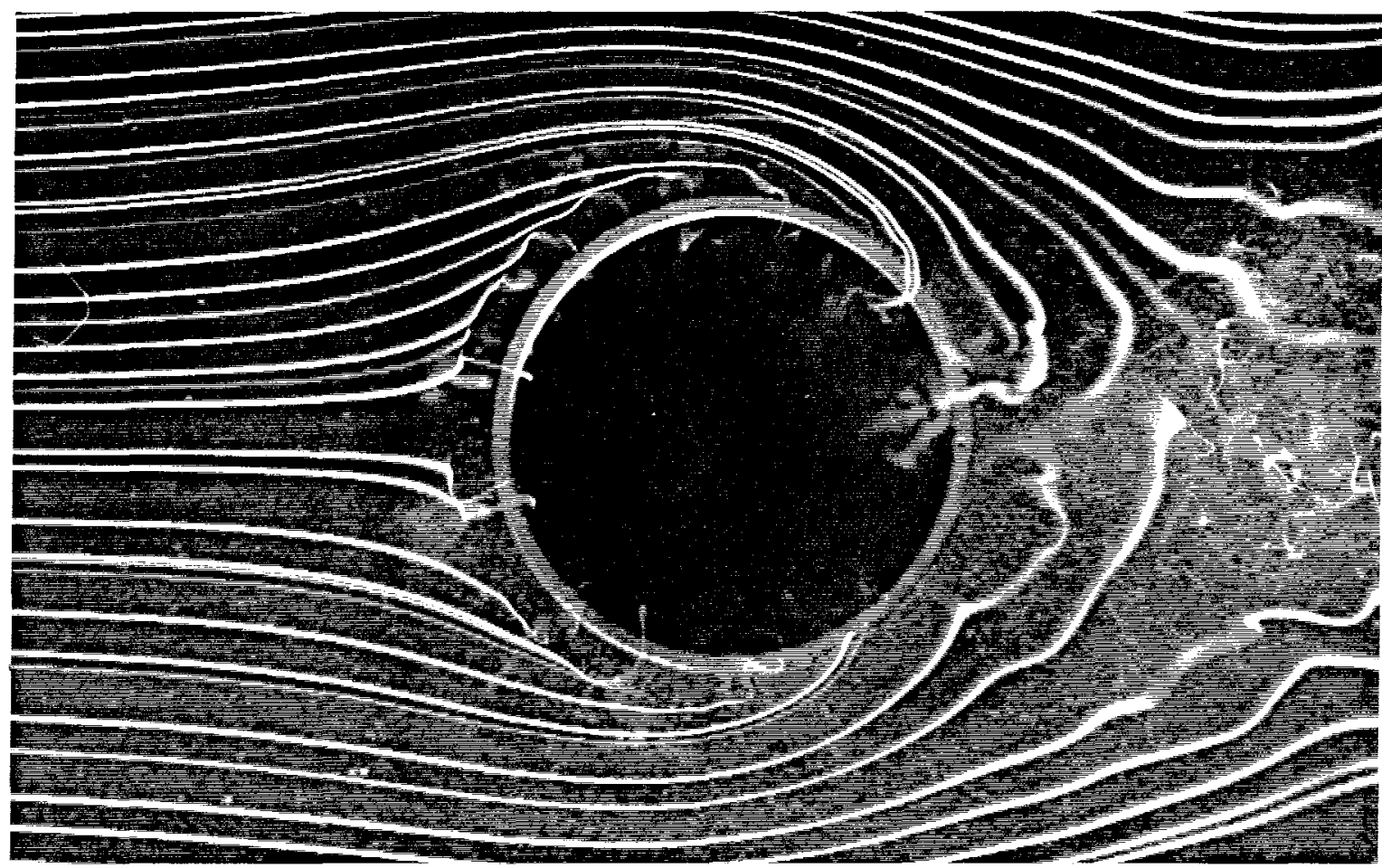

(b) $V R=10$.

FIGURE 3.3: Streakline pattern with the jet issuing at the viewer shows entrainment of crossflow fluid. (a) $V R=2 . R e_{c f}=7600 . Z_{s w} / D_{j}=.75$, (b) $V R=10 . R e_{c f}=3800 . Z_{s w} / D_{j}=.5$. 


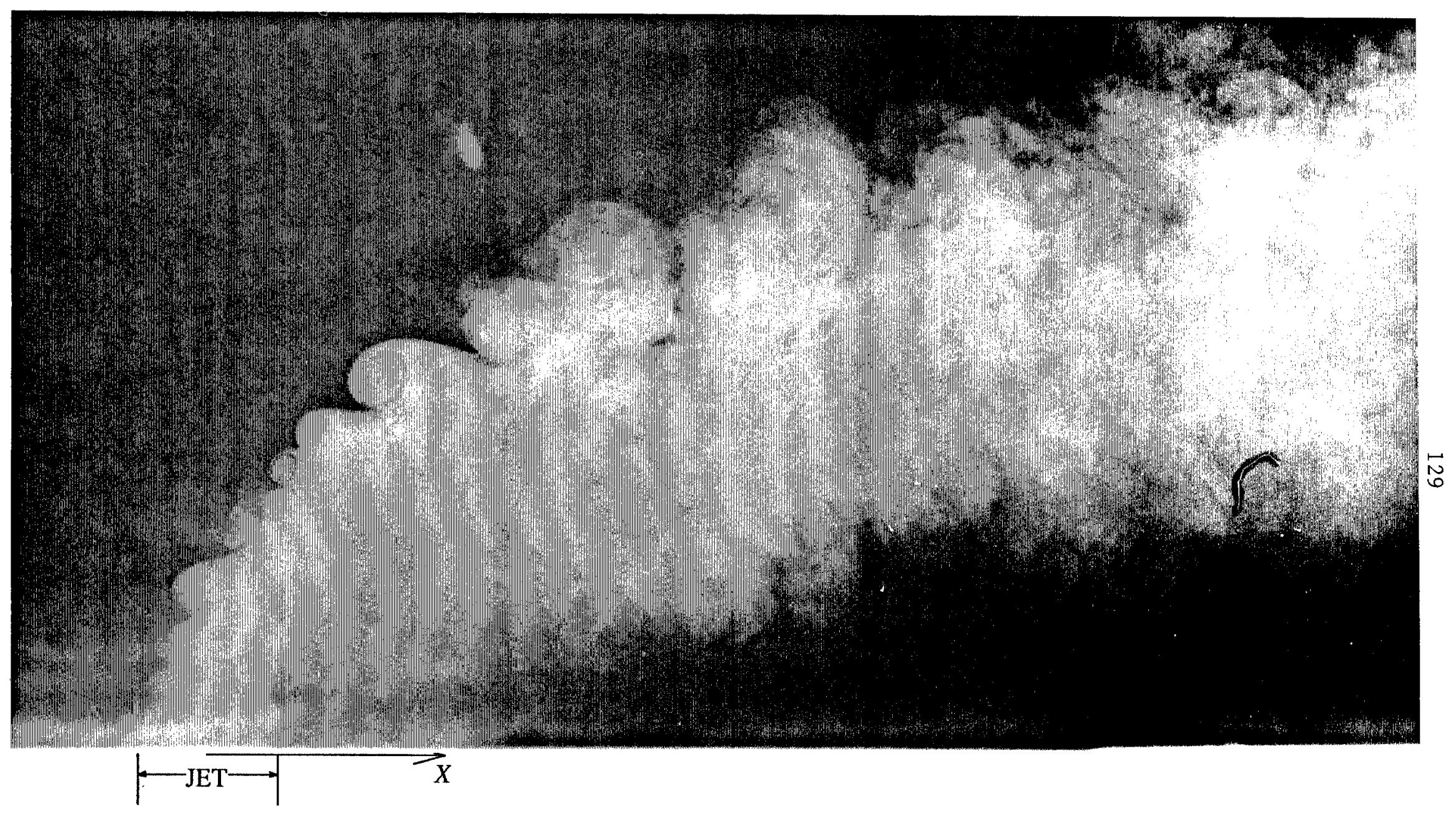

FIGURE 3.4a: $V R=2$.

FIGURE 3.4: Side view of the deflected jet. (a) $V R=2$, (b) $V R=4$, (c) $V R=6$, (d) $V R=8$, (e) $V R=10$. $R e_{c f}=3800$. 


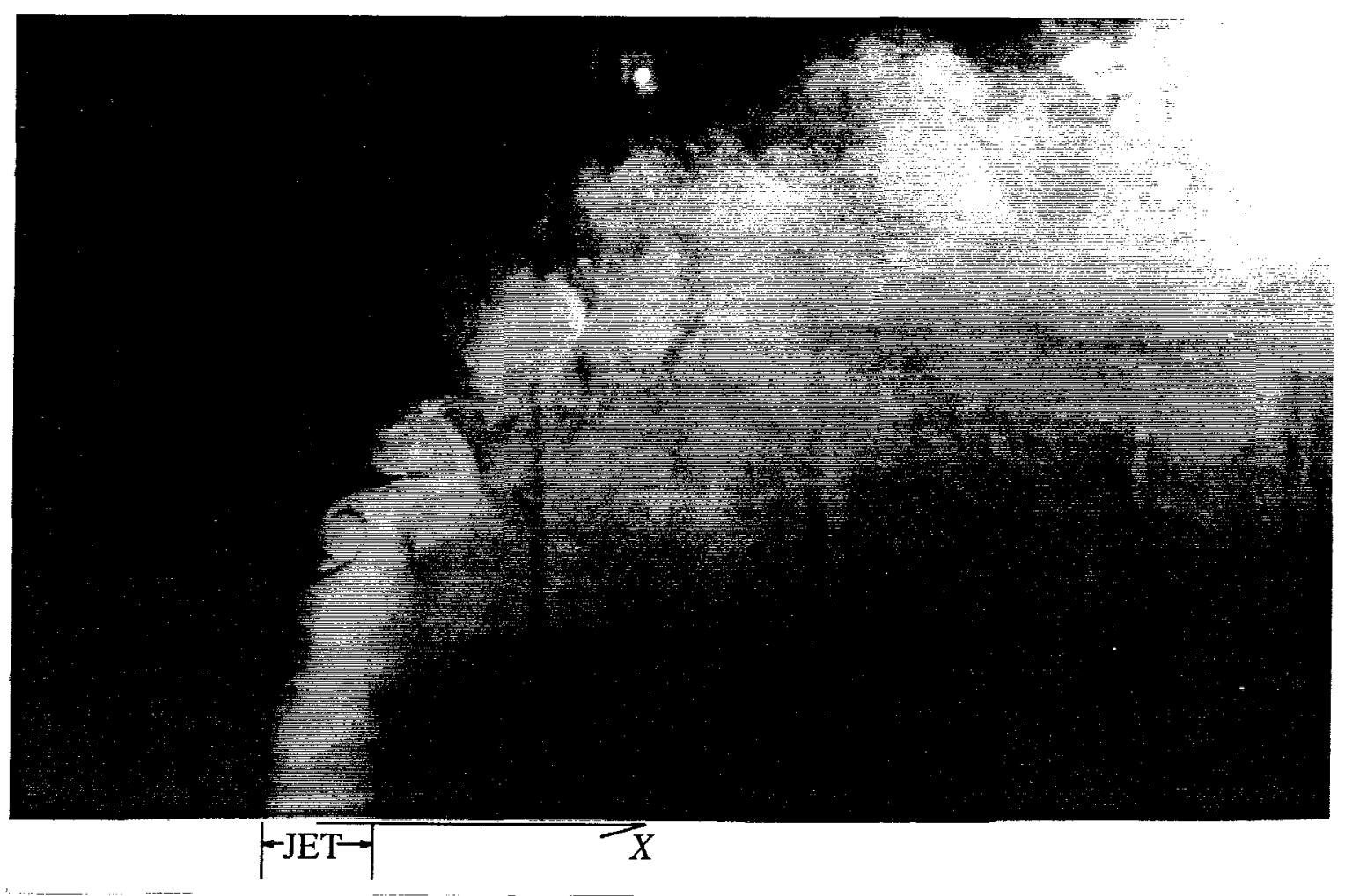

FIGURE 3.4b: $V R=4$.

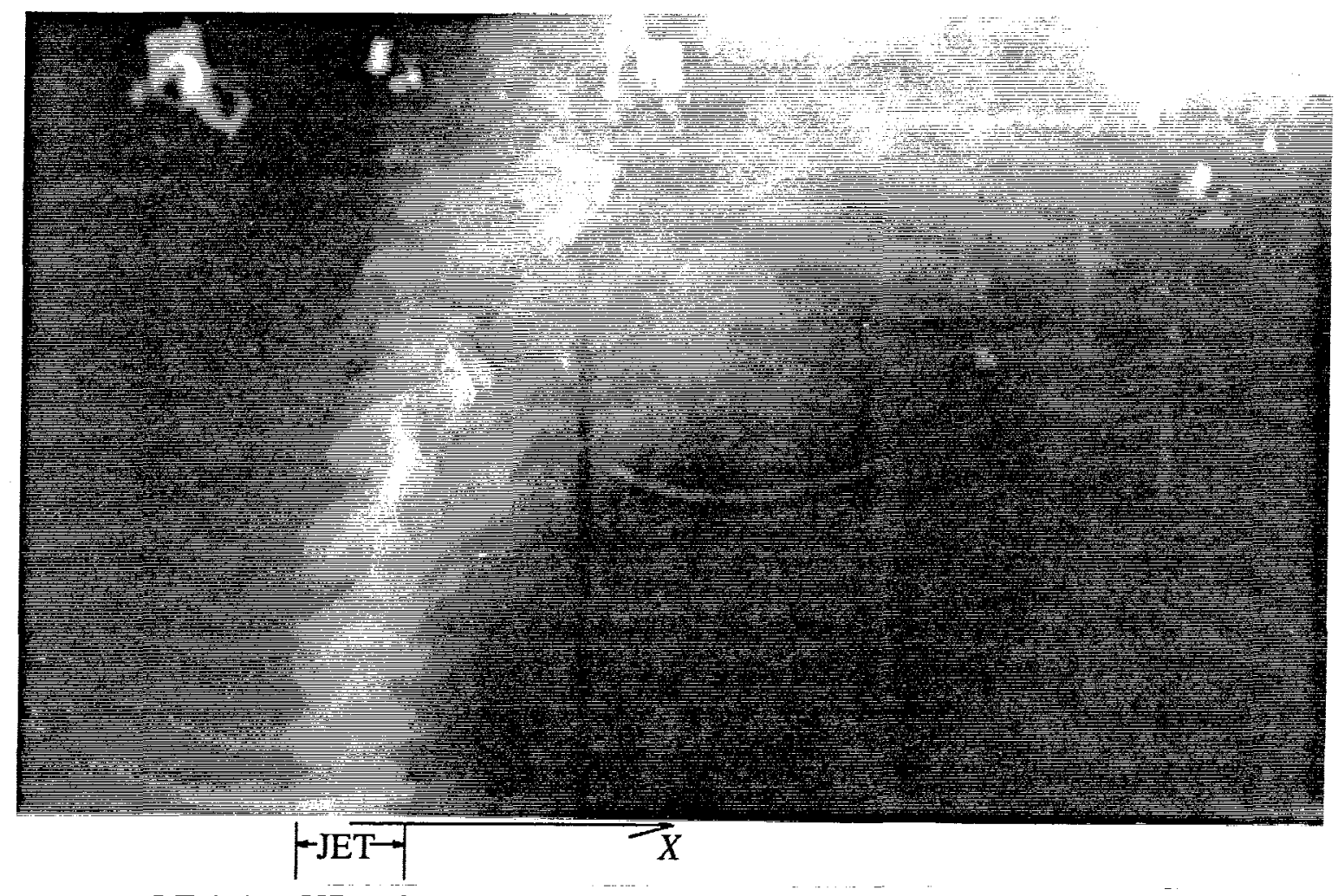

FIGURE 3.4c: $V R=6$. 


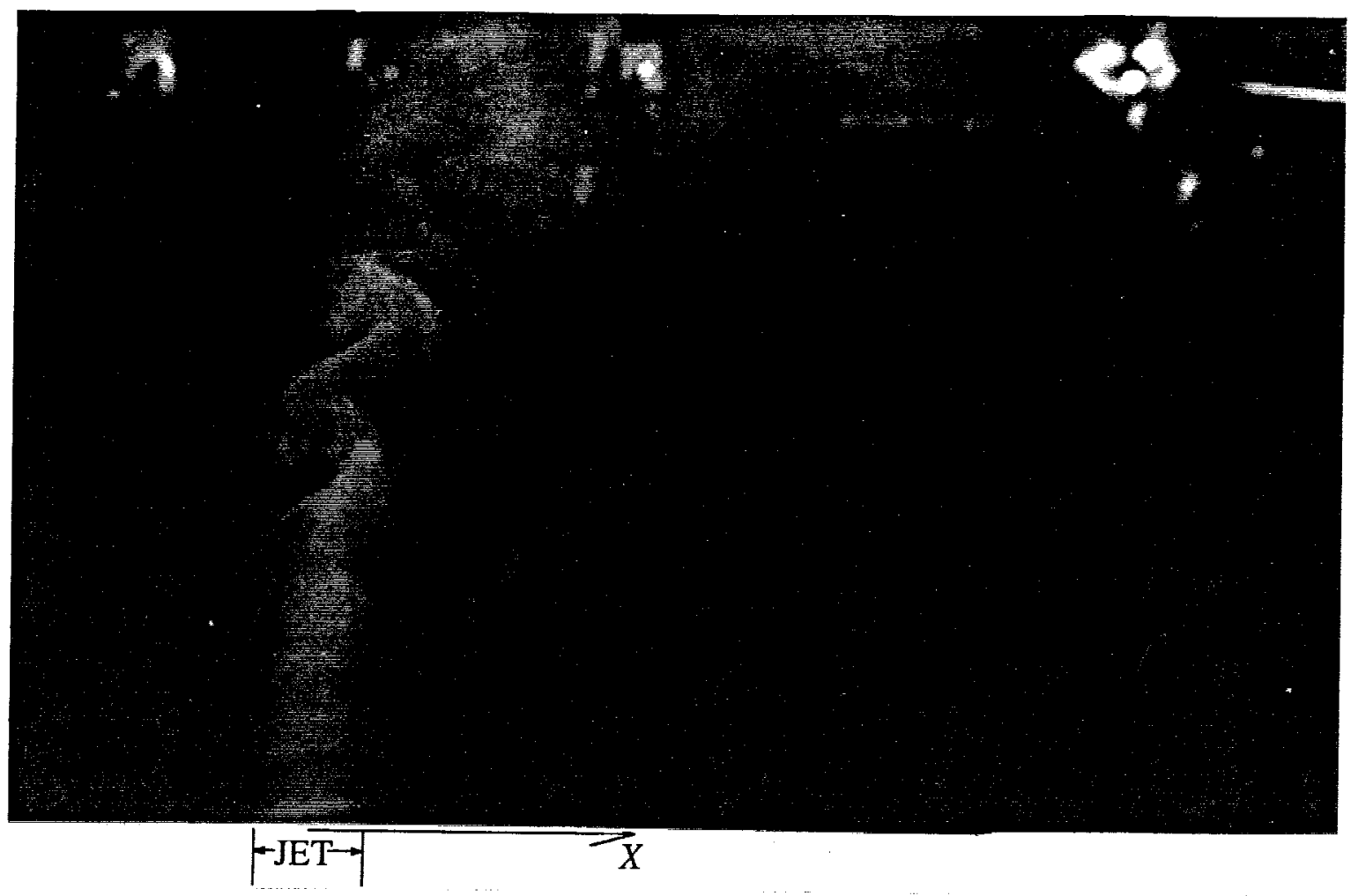

FIGURE 3.4d: $V R=8$.

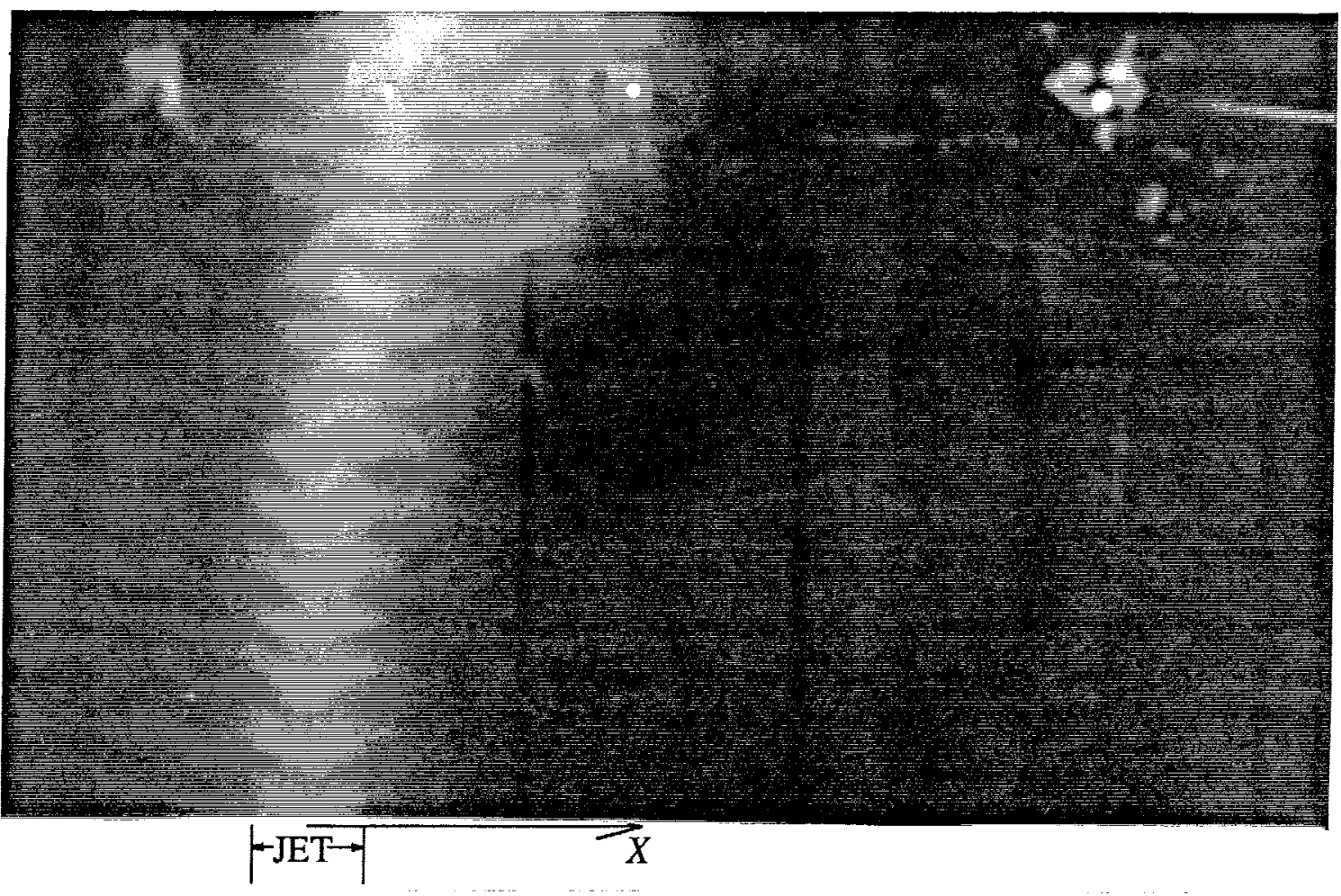

FIGURE 3.4e: $V R=10$. 


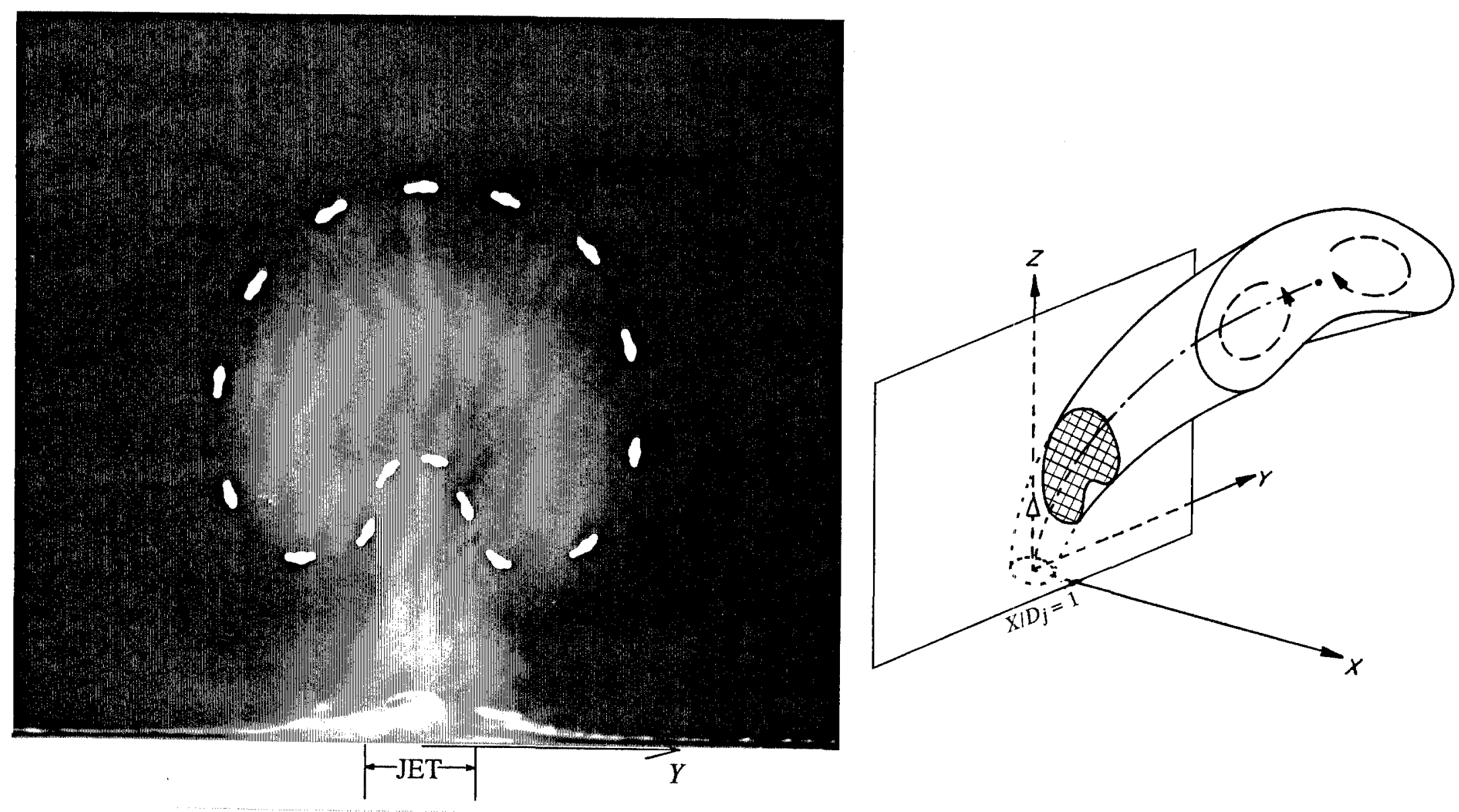

FIGURE 3.5: Section at $X / D_{j}=1$ shows early development of the counterrotating vortex pair, as viewed from $X / D_{j}>1$. The drawing at right indicates the section visualized. $V R=4 . R e_{c f}=3800 . Z_{s w} / D_{j}=0+$. 


\section{3}
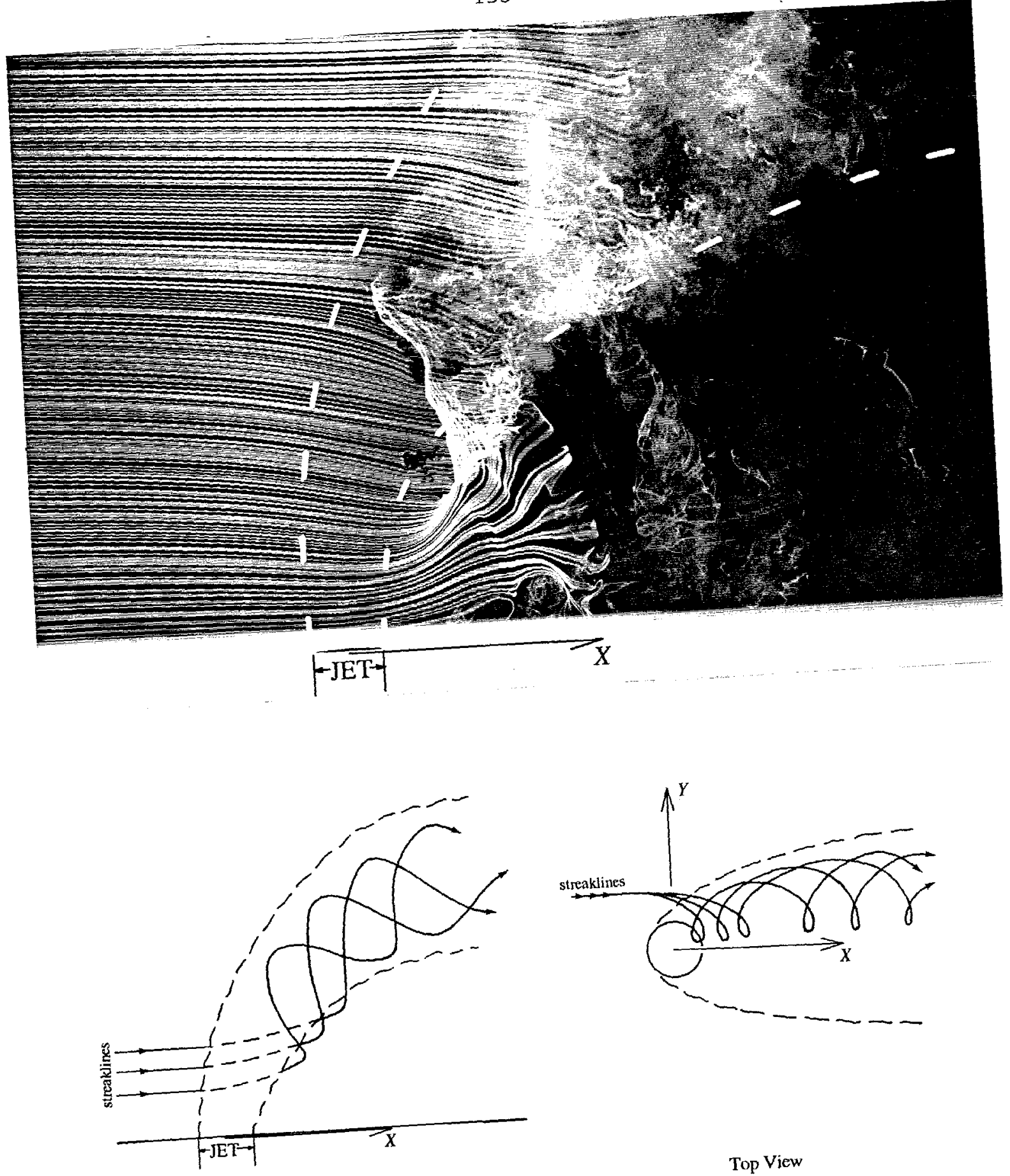

Side View

Top View

FIGURE 3.6: Streaklines showing entrainment of crossflow fluid by the vortex pair. The drawing clarifies the flow pattern. $V R=6 . R e_{c f}=3800$. $Y_{s w} J D_{j}=1$. 


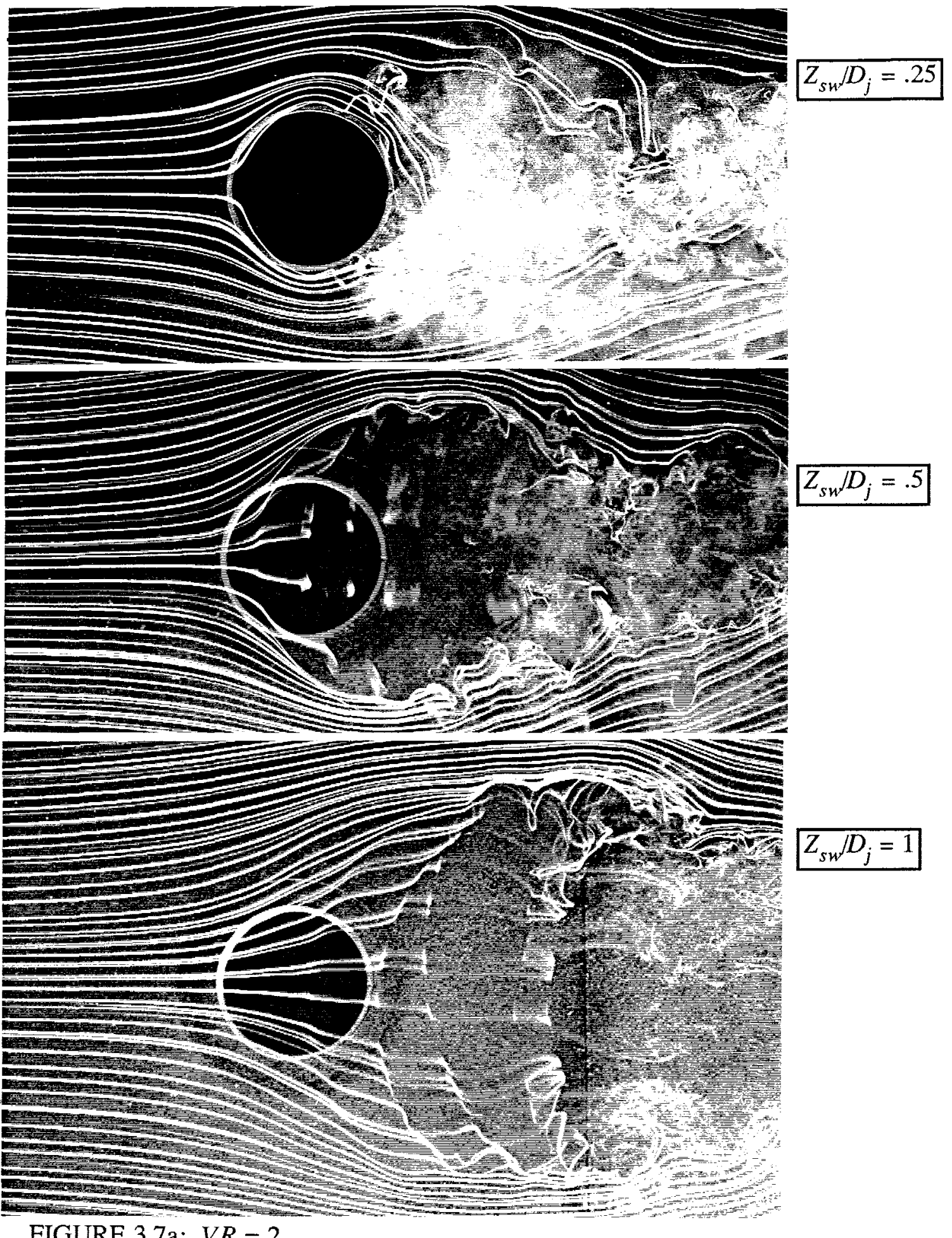

FIGURE 3.7: Development of the jet cross section with distance from the crossflow wall. Jet issues at the viewer. (a) $V R=2$. $R e_{c f}=3800$, (b) $V R=4$. $R e_{c f}=3800$, (c) $V R=6 . R e_{c f}=11400$, (d) $V R=8$. $R e_{c f}=3800,(\mathrm{e}) V R=10 . R e_{c f}=7600$. 
135

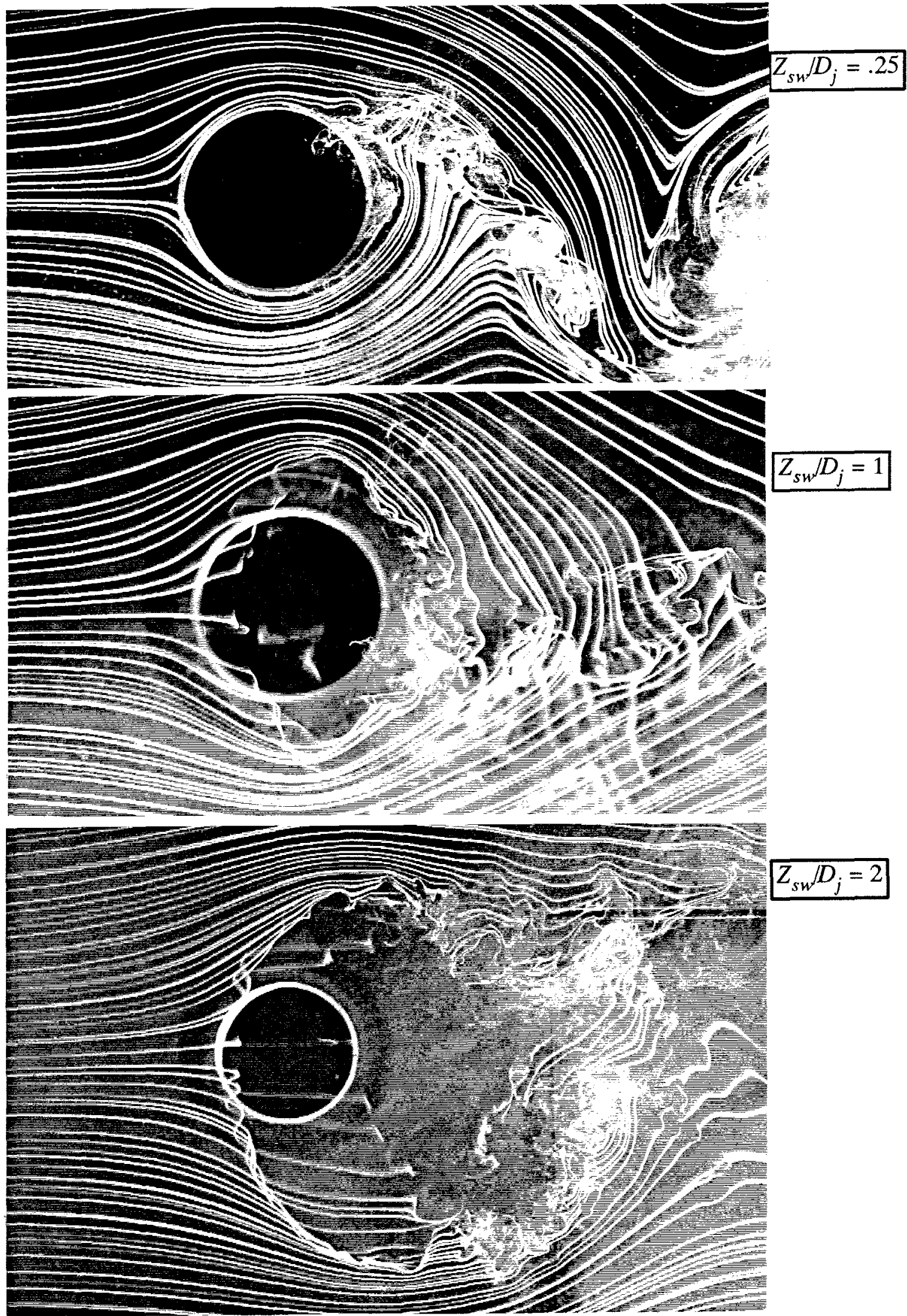

FIGURE 3.7b: $V R=4$. 

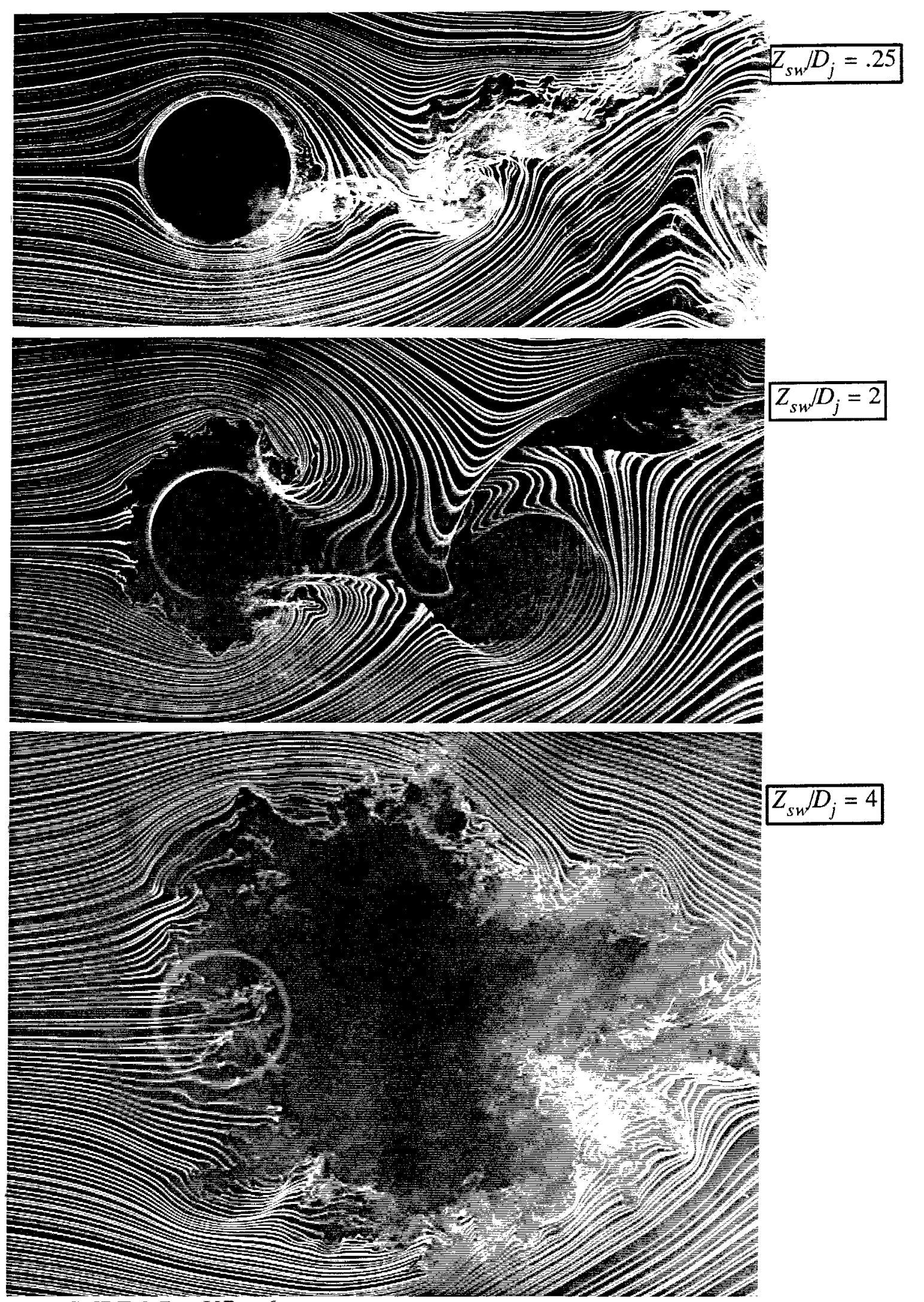

FIGURE 3.7c: $V R=6$. 


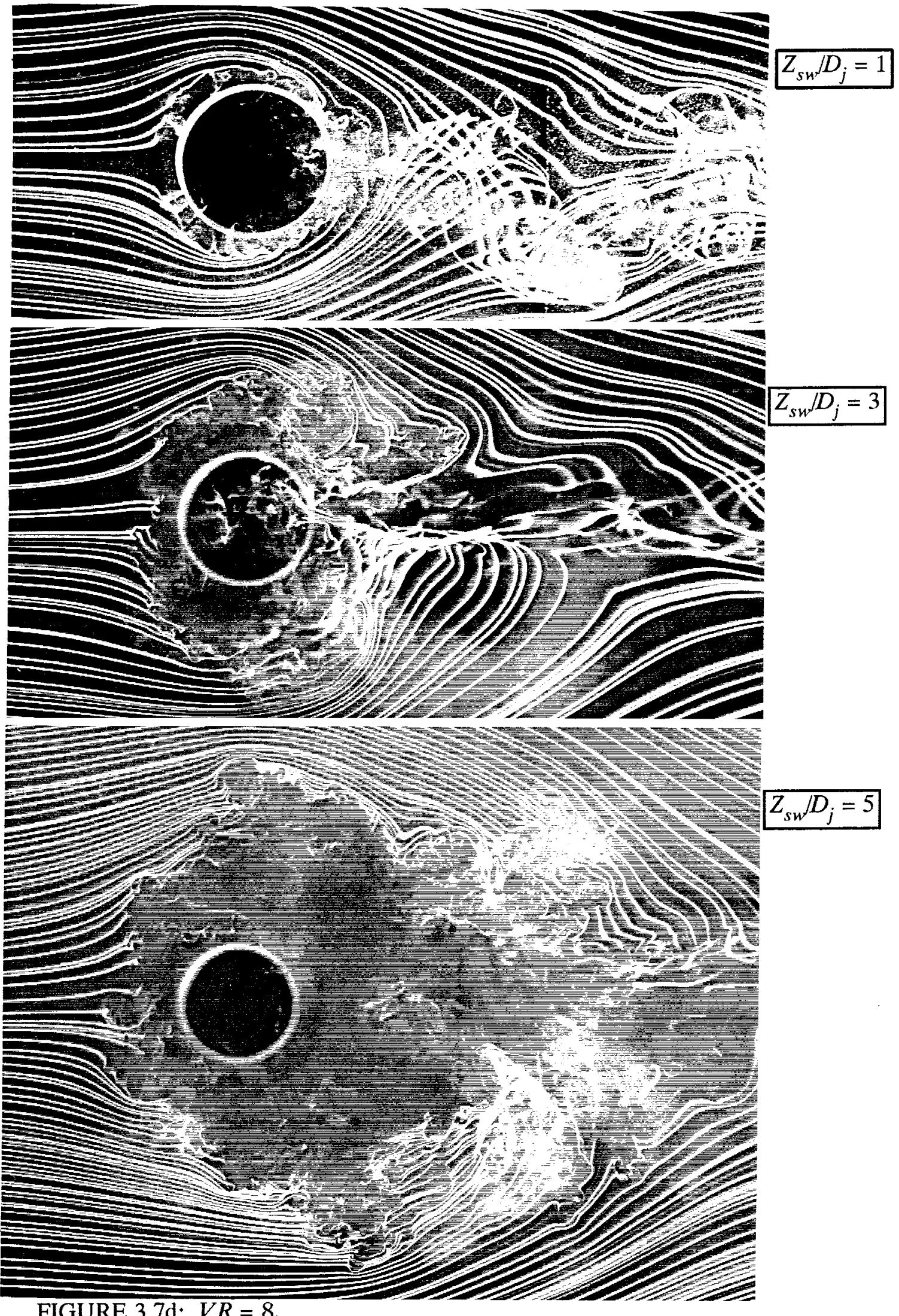



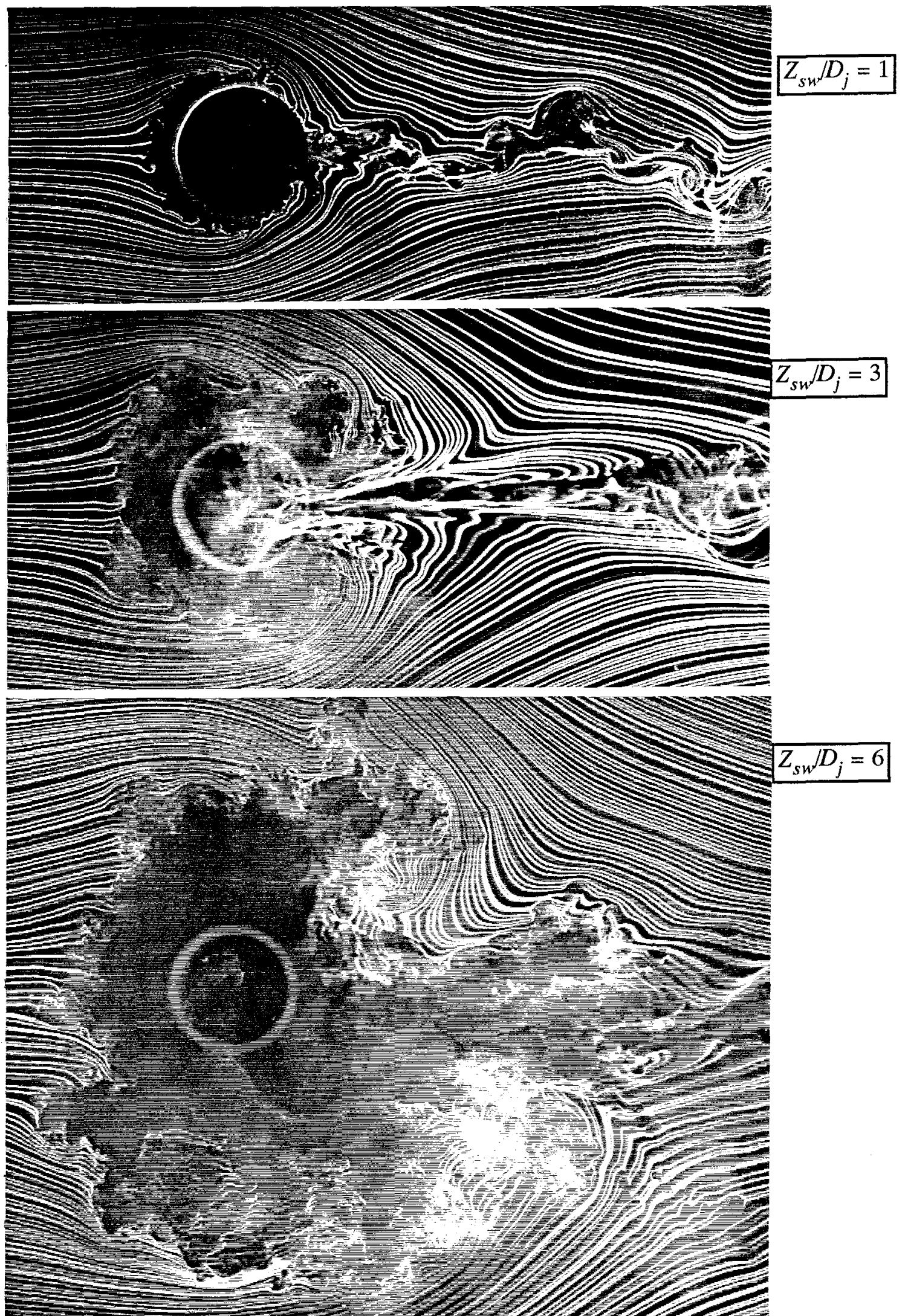

FIGURE 3.7e: $V R=10$. 

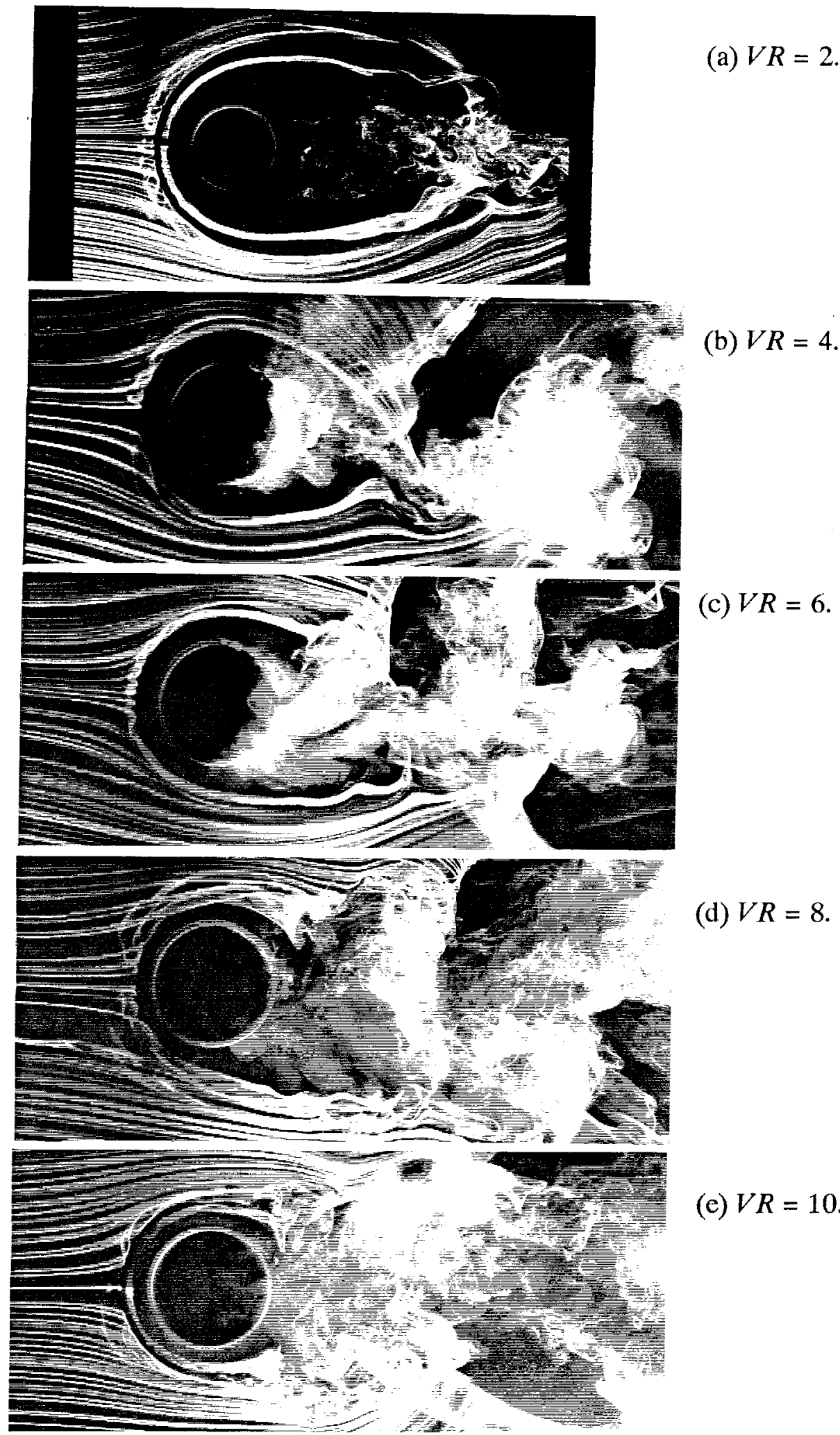

FIGURE 3.8: Horseshoe vortex system at the crossflow wall. Jet issues at the viewer. (a) $V R=2$, (b) $V R=4$, (c) $V R=6$, (d) $V R=8$, (e) $V R=10$. $R e_{c f}=3800 . Z_{\mathrm{sw}} / D_{j}=0+$.
(a) $V R=2$.

(b) $V R=4$.

(c) $V R=6$.

(e) $V R=10$ 


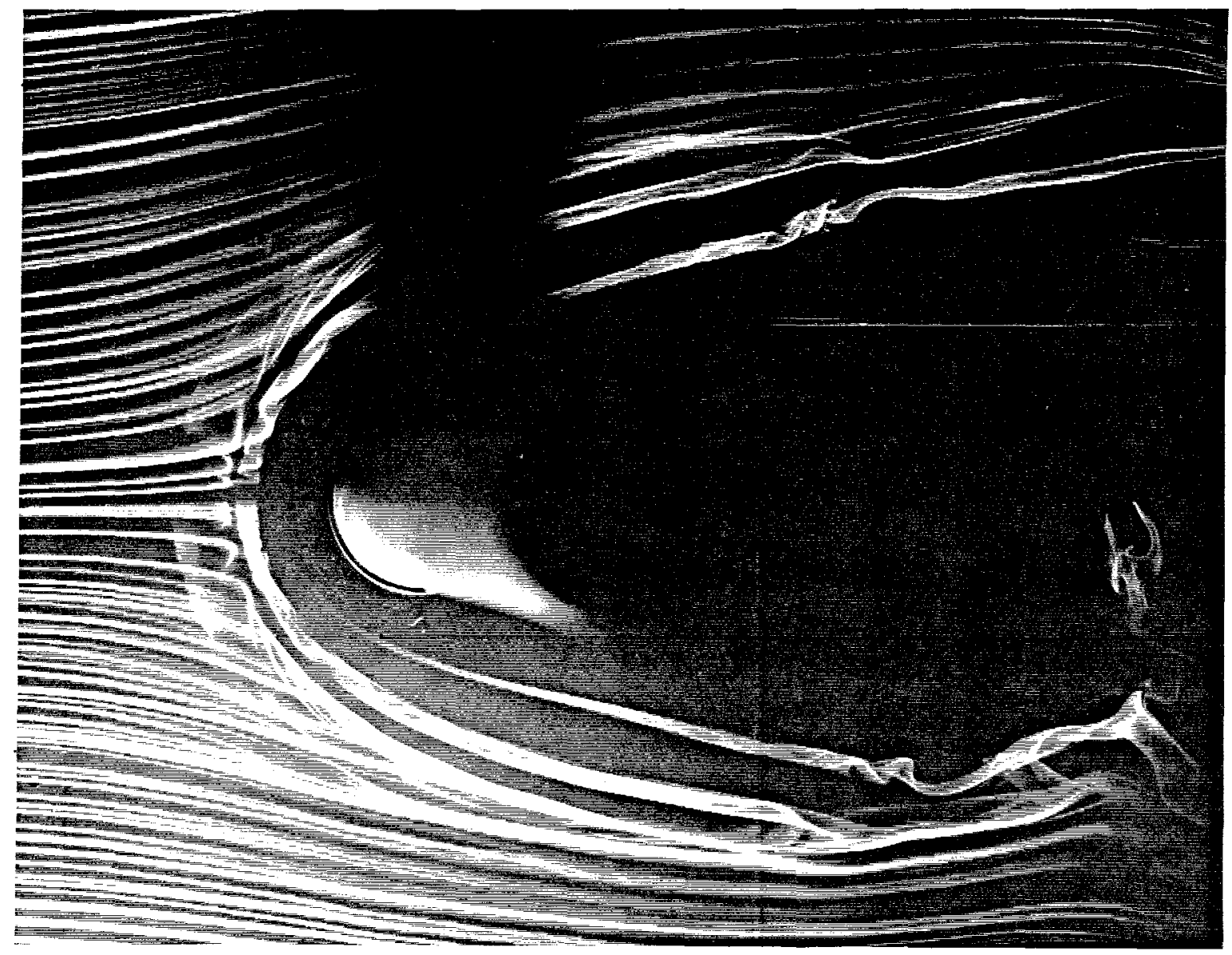

FIGURE 3.9: Horseshoe vortex system for a wall-mounted circular cylinder of $A R=6 . R e_{c f}=3800 . Z_{s w} / D_{j}=0+$. 


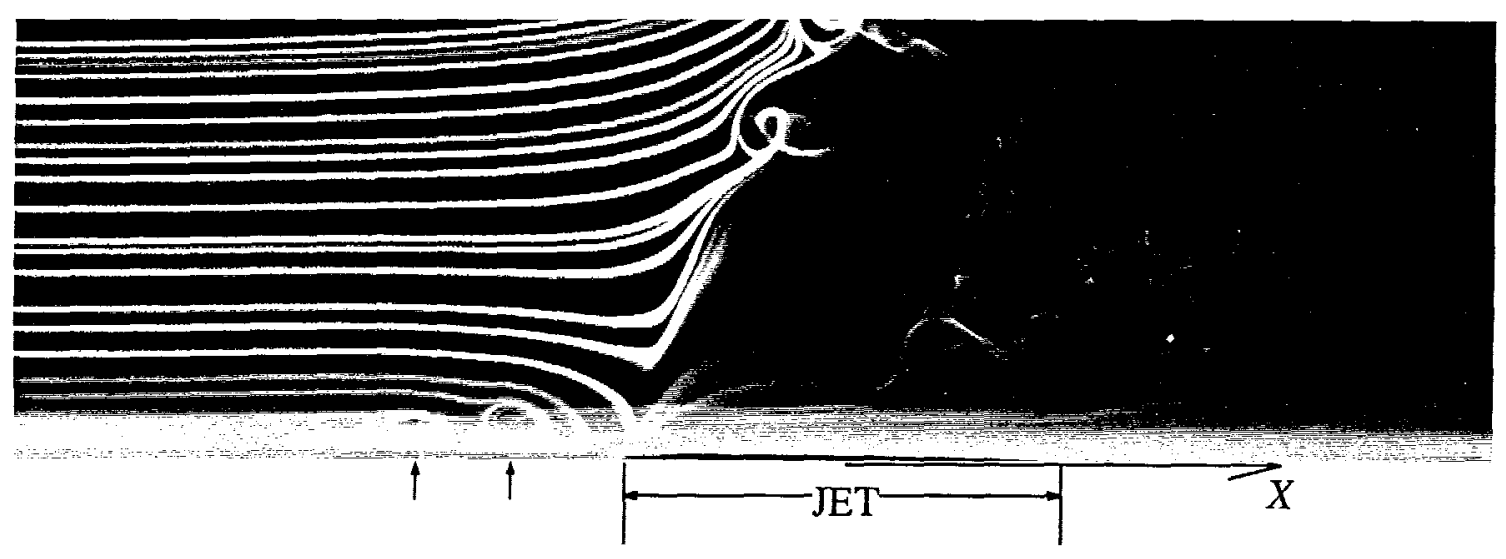

(a) $V R=2$.

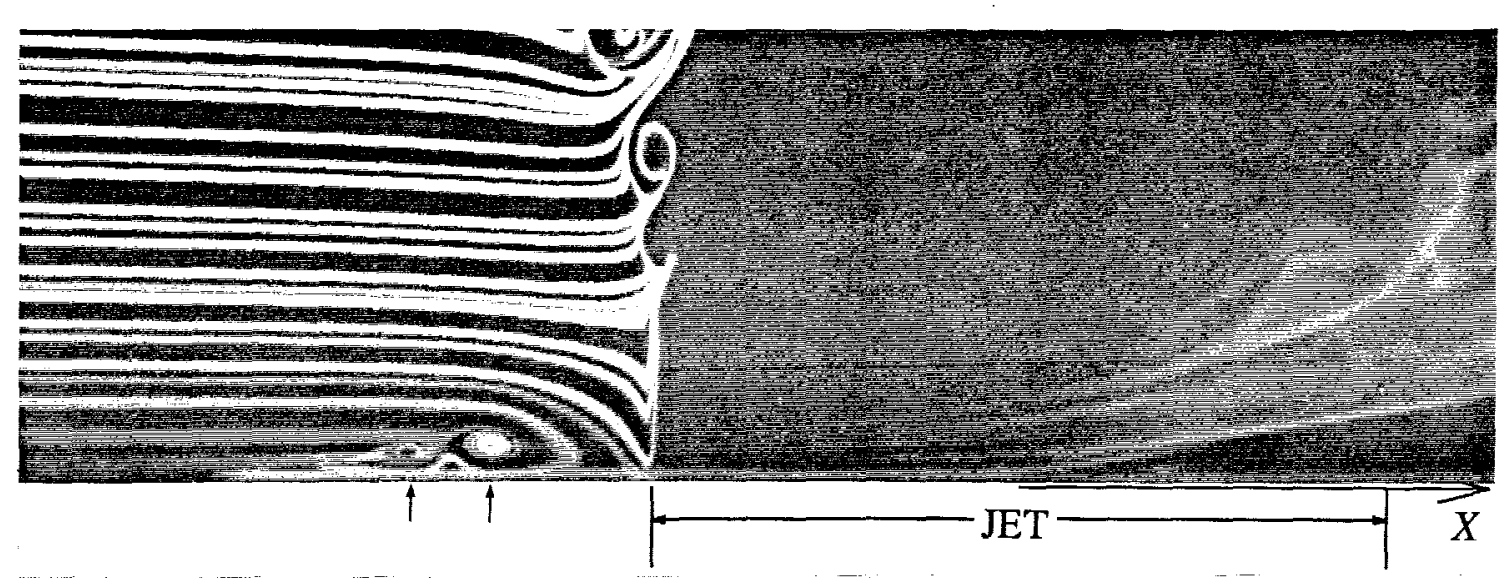

(b) $V R=10$.

FIGURE 3.10: $Y / D_{j} \approx 0$ cross section of the horseshoe vortices just ahead of the jet. (a) $V R=2$, (b) $V R=10 . R e_{c f}=3800 . Y_{s w} / D_{j}=0$. 


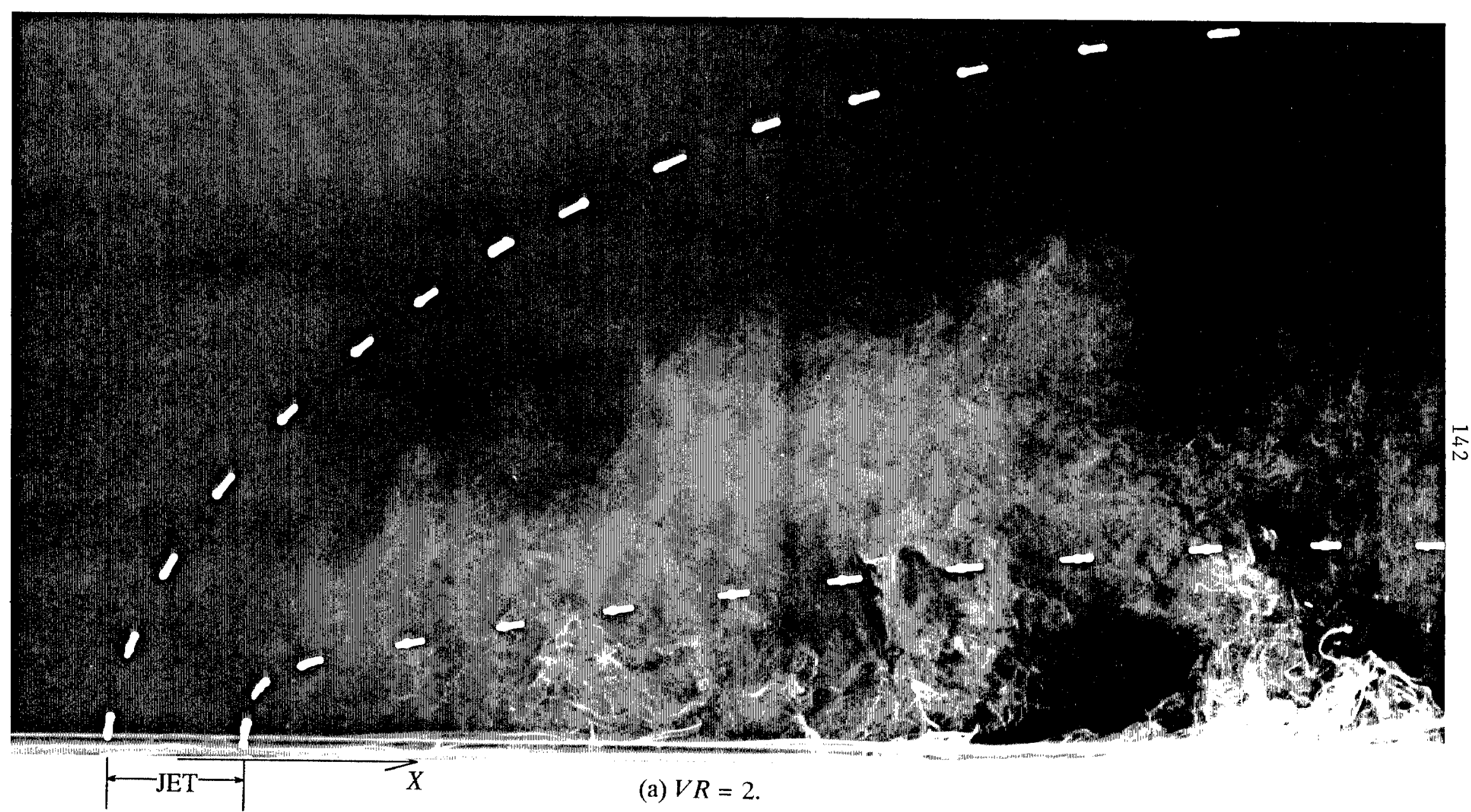

FIGURE 3.11: Side view of the wake vortices, which extend from the crossflow wall to the jet. (a) $V R=2$, (b) $V R=4$, (c) $V R=6$, (d) $V R=8$, (e) $V R=10 . R e_{c f}=3800 . Z_{s h} J D_{j}=0+$. 


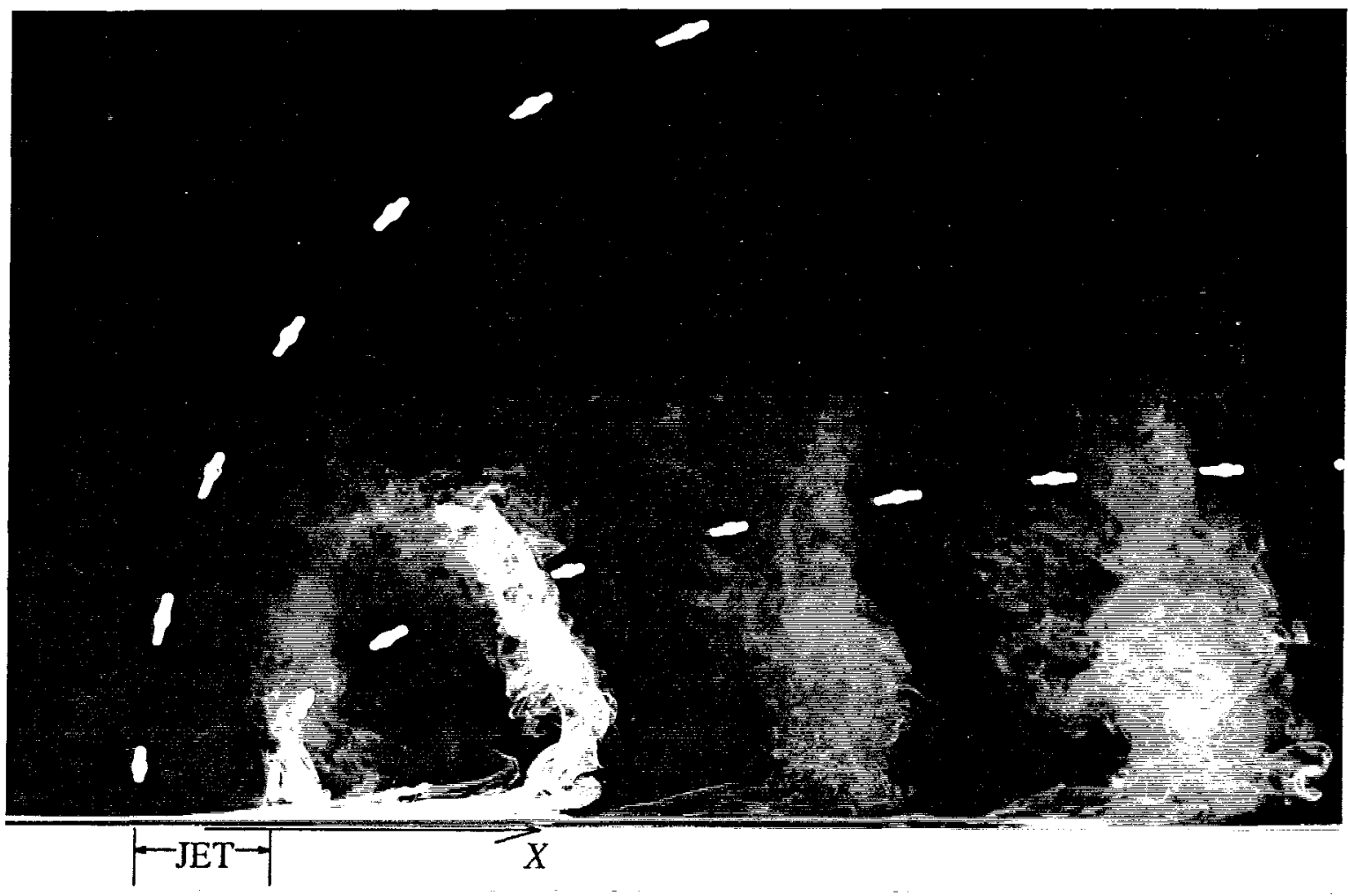

FIGURE 3.11b: $V R=4$.

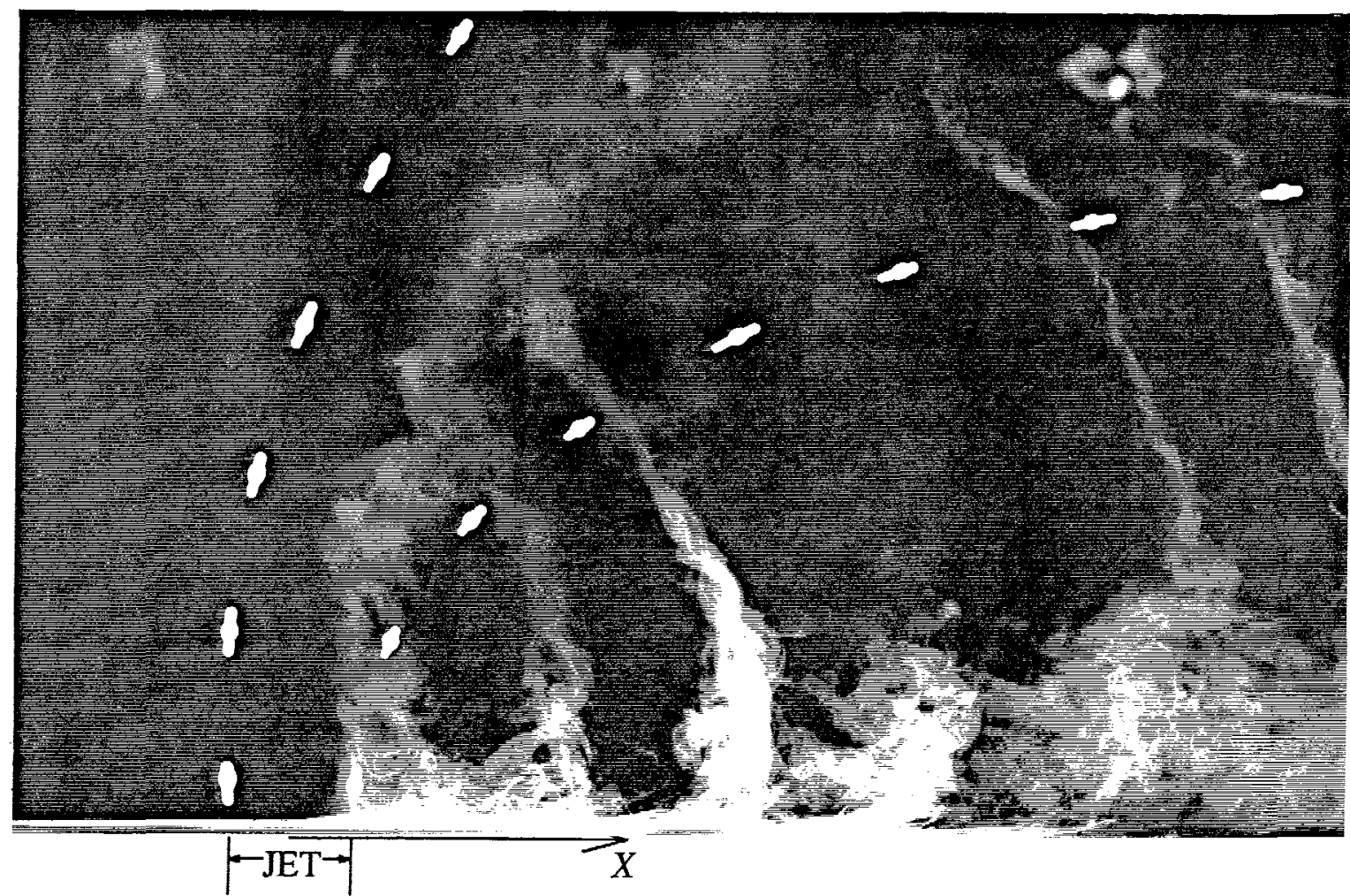

FIGURE 3.11c: $V R=6$. 


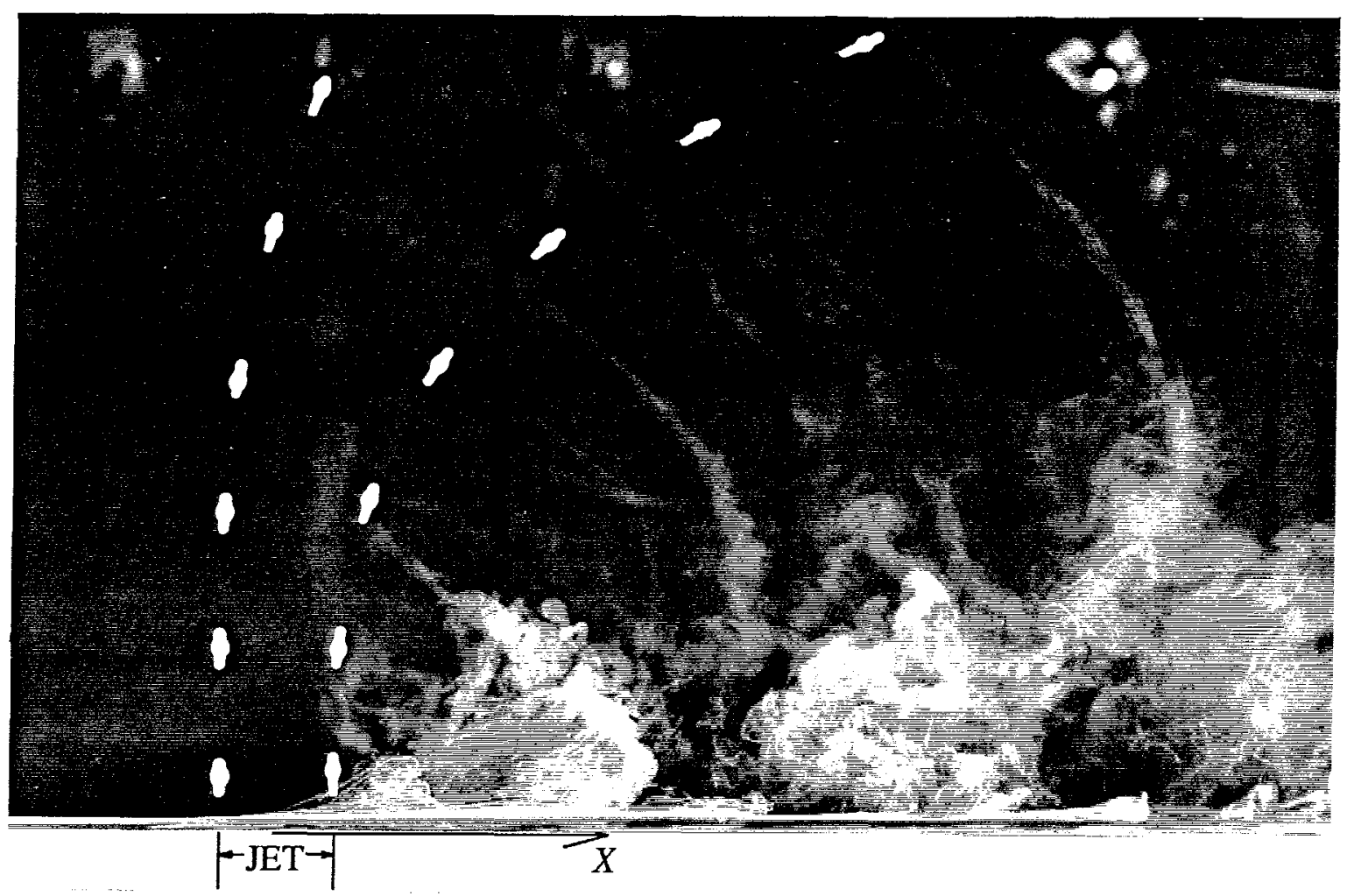

FIGURE 3.11d: $V R=8$.

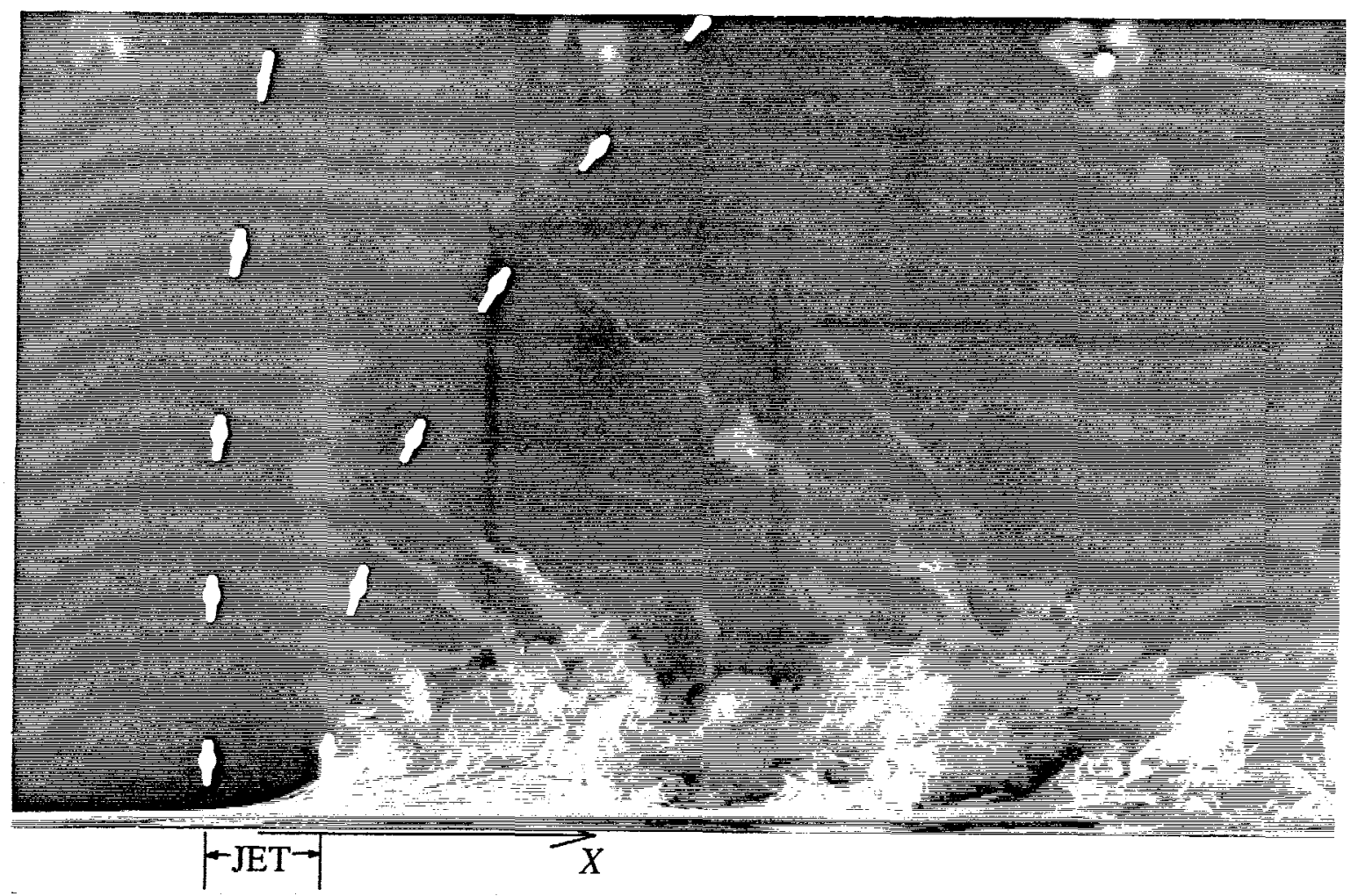

FIGURE 3.11e: $V R=10$. 


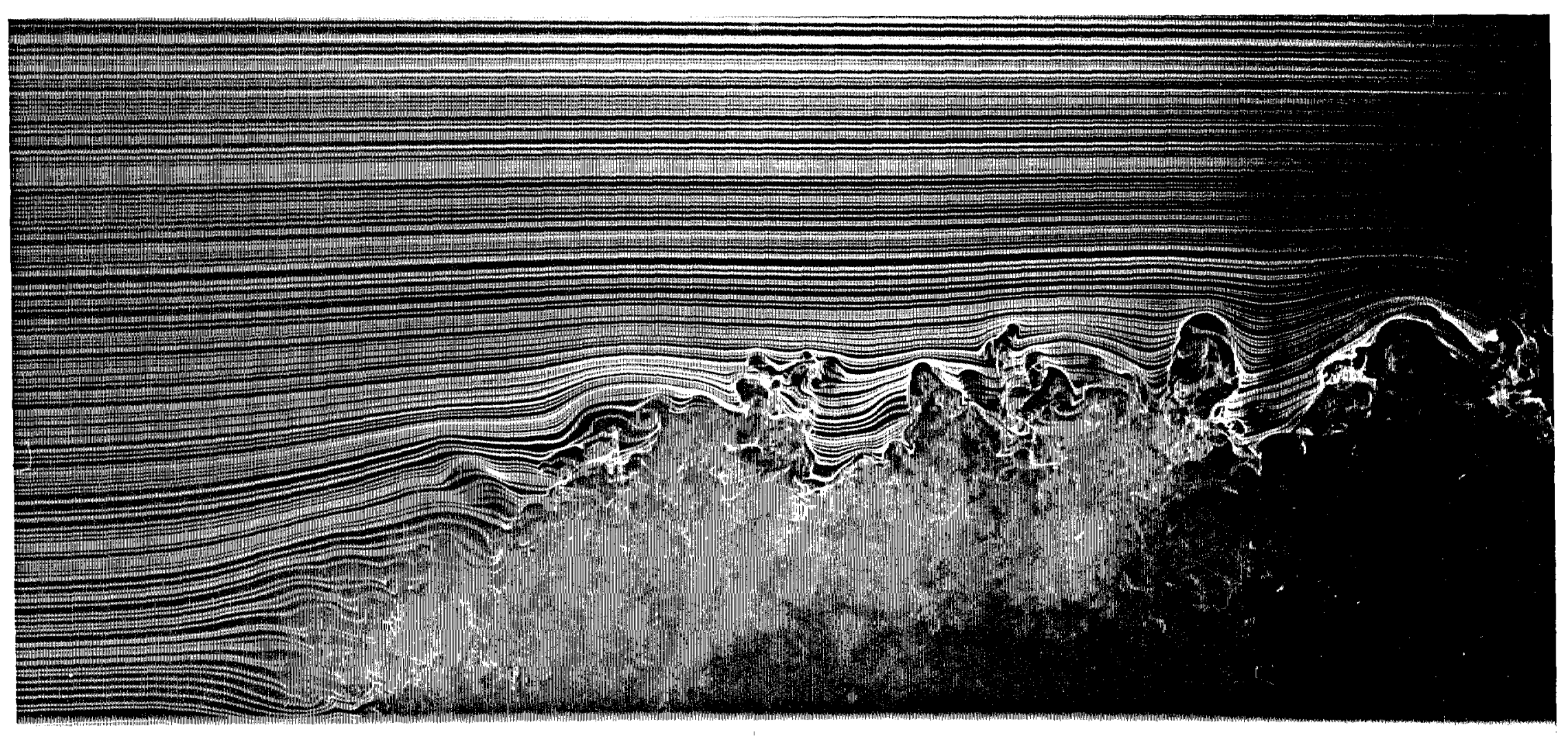

$$
\begin{array}{lll}
-\mathrm{JET} \rightarrow & \text { (a) } V R=2 .
\end{array}
$$

FIGURE 3.12: Entrainment of crossflow fluid by the wake structures.
(a) $V R=2 . Y_{s w} D D_{j}=.5$, (b) $V R=4 . Y_{s w} / D_{j}=1.5$,
(c) $V R=6 . Y_{s w} / D_{j}=1$, (d) $V R=8 . Y_{s w} / D_{j}=1$,
(e) $V R=10 . Y_{s w} J D_{j}=1$. $R e_{c f}=3800$. 


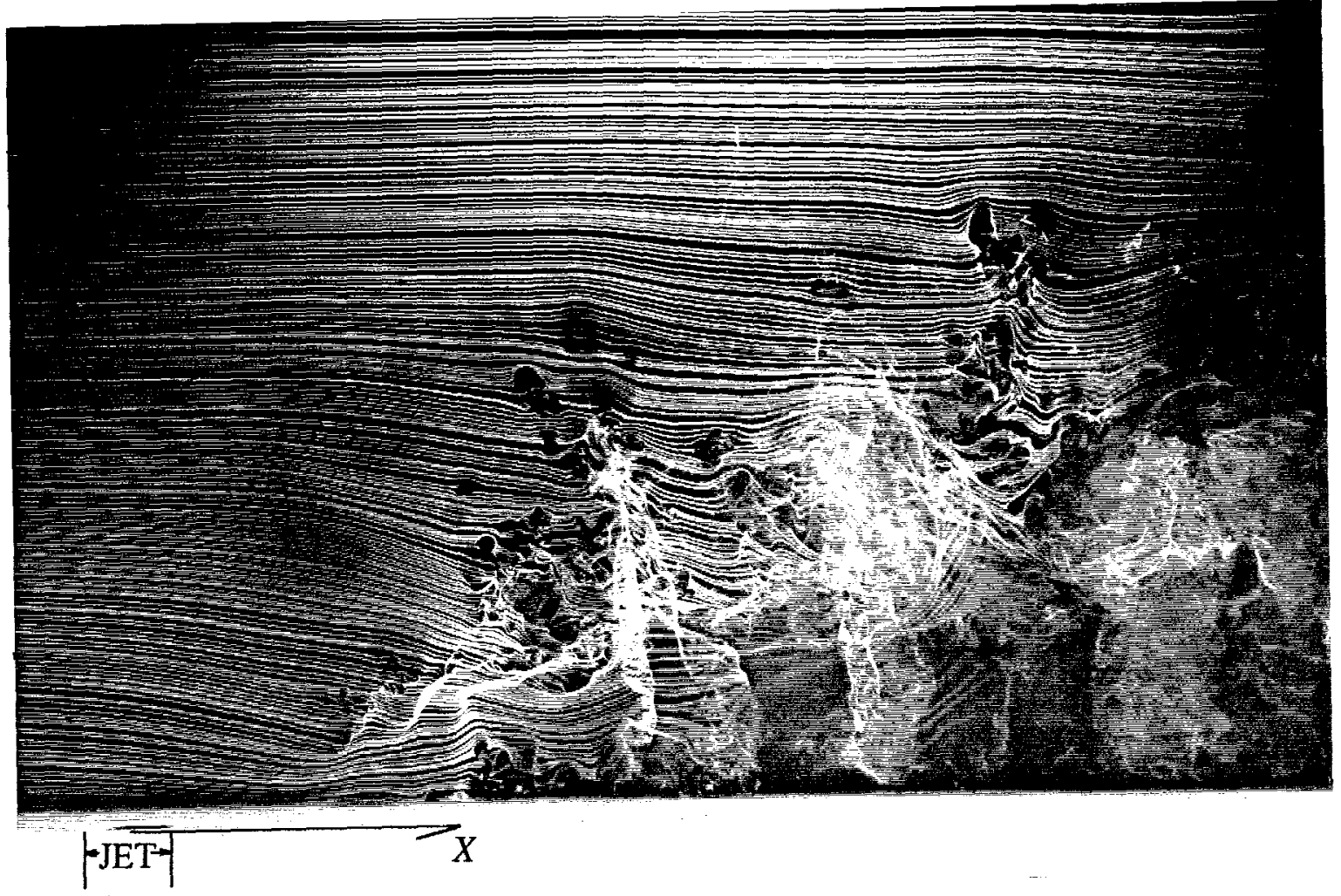

FIGURE 3.12b: $V R=4$.
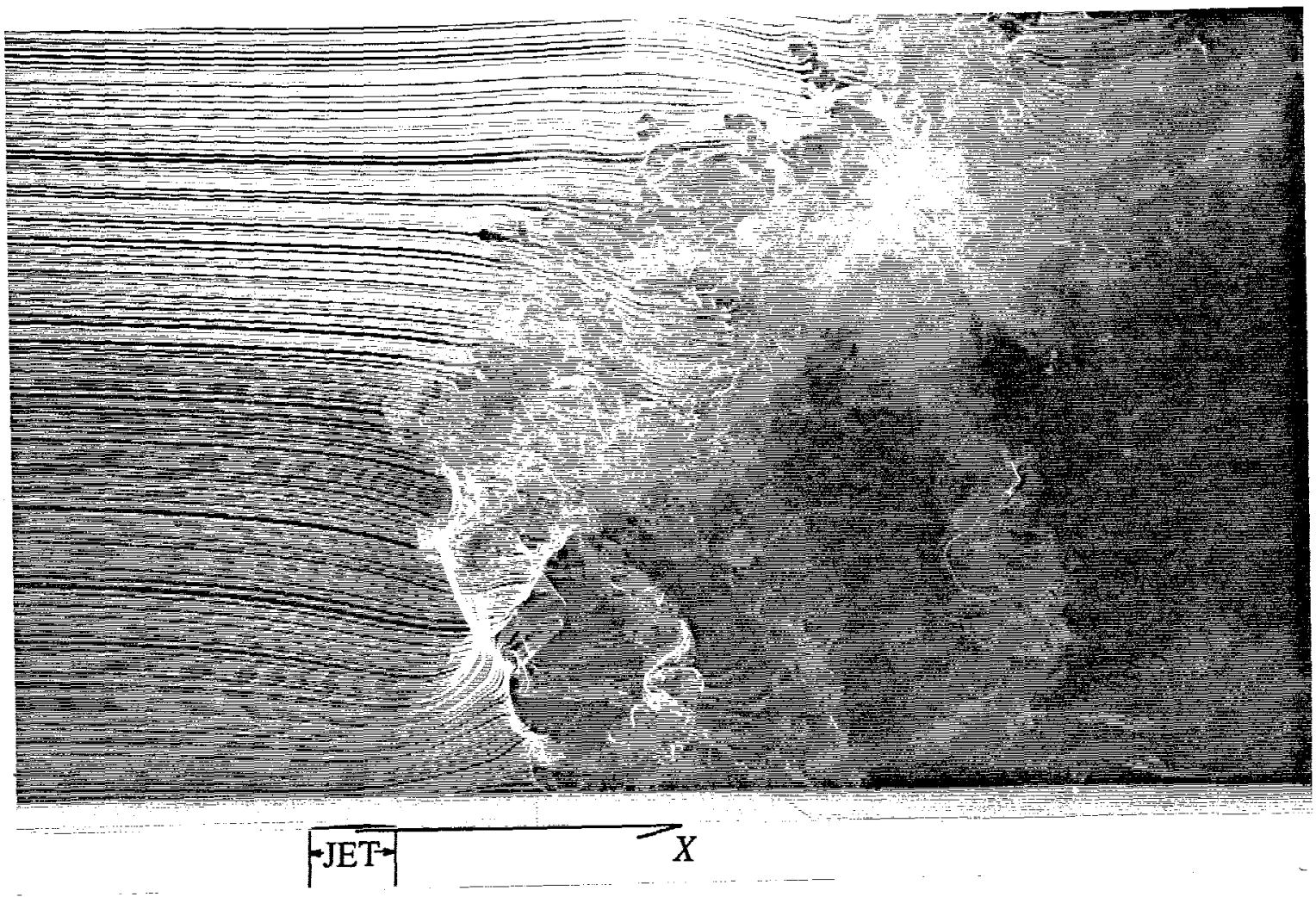

FIGURE 3.12c: $V R=6$. 


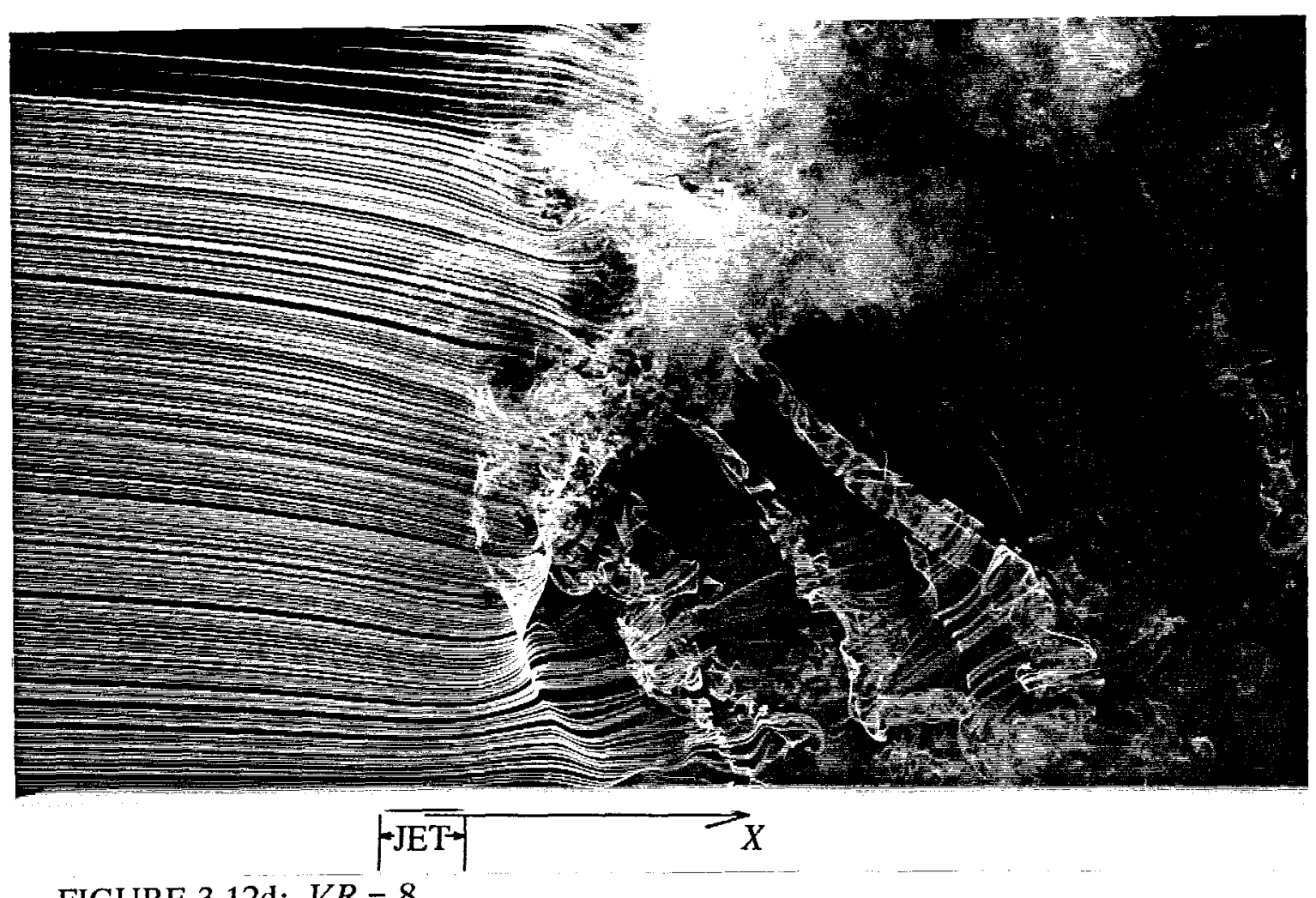

FIGURE 3.12d: $V R=8$.

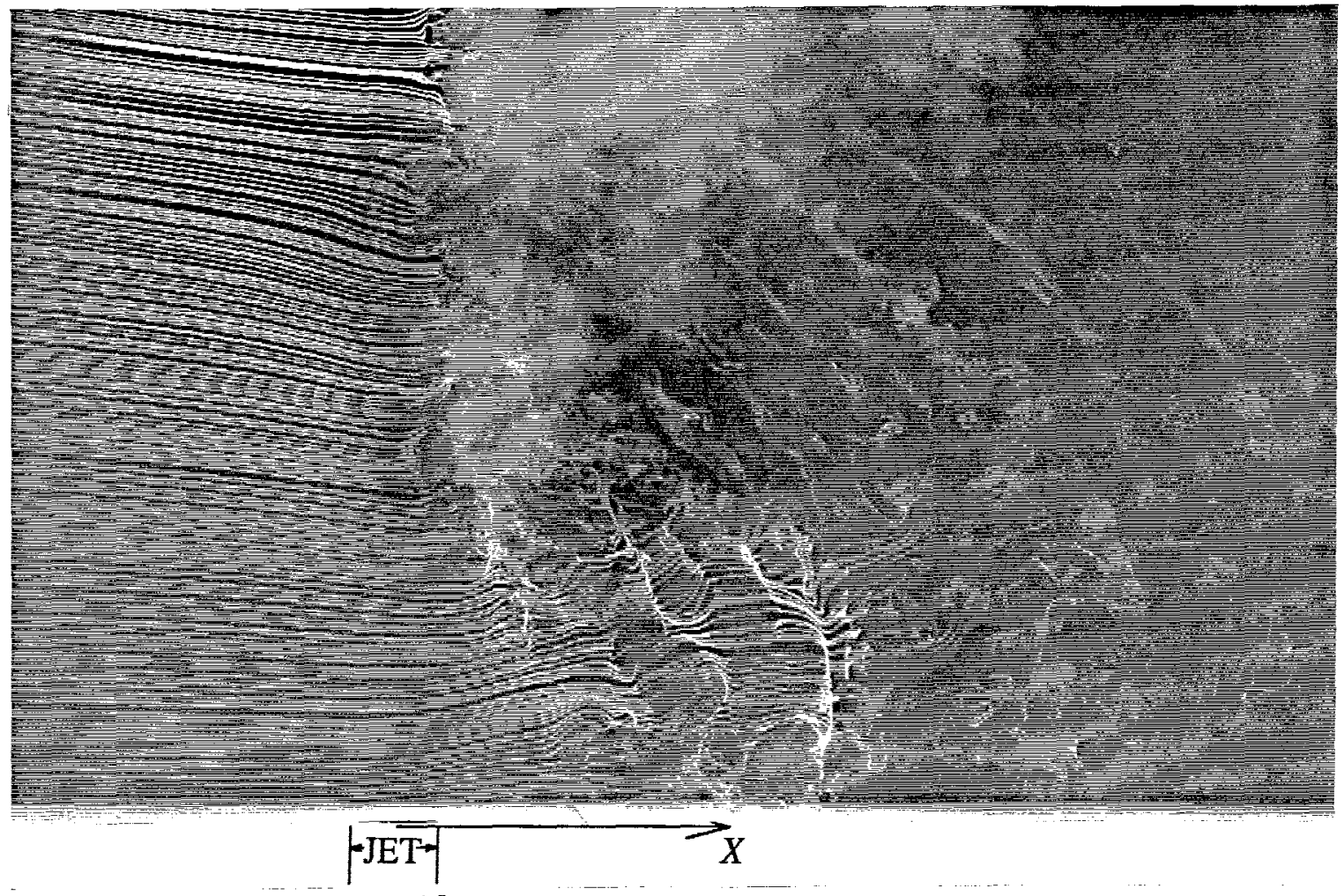

FIGURE 3.12e: $V R=10$. 


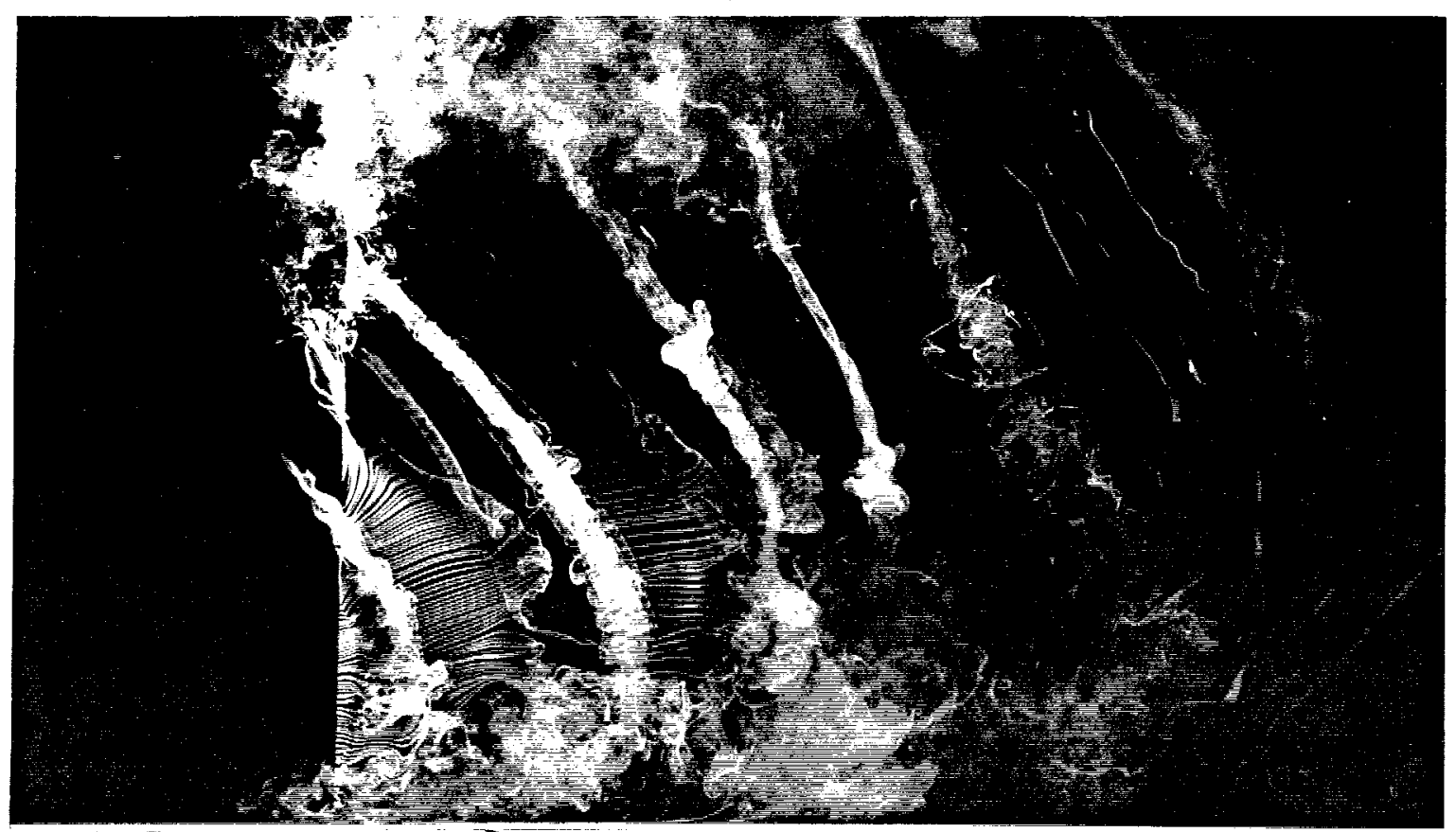

HET $\Rightarrow$ Smoke-wire $\vec{X}$

(a) $V R=8$.

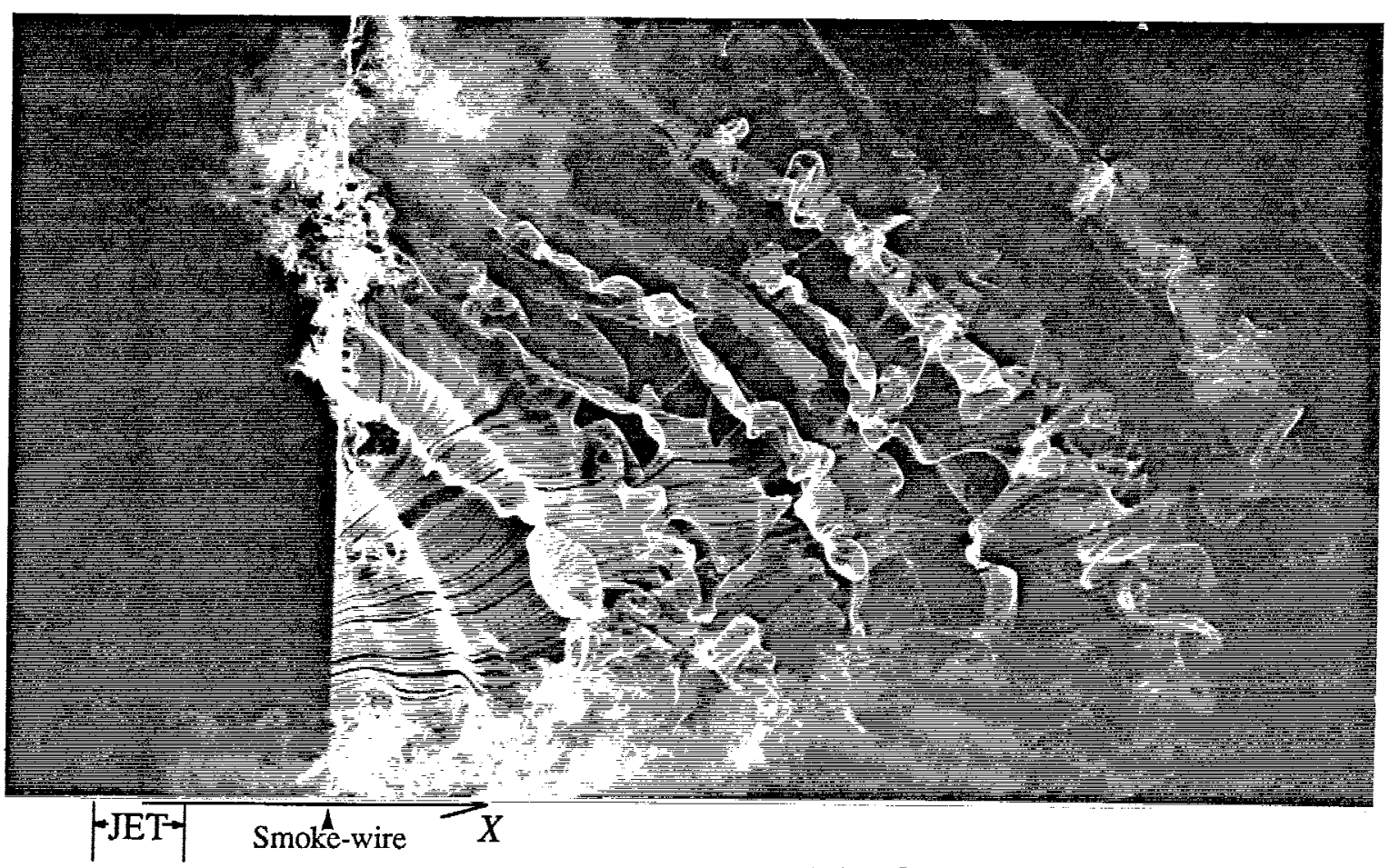

(b) $V R=10$.

FIGURE 3.13: Side view of wake vortices. (a) $V R=8 . Y_{s w} / D_{j}=-.5$, (b) $V R=10$.

$$
Y_{s w} / D_{j}=.5 . R e_{c f}=3800 . X_{s w} / D_{j}=2 \text {. }
$$




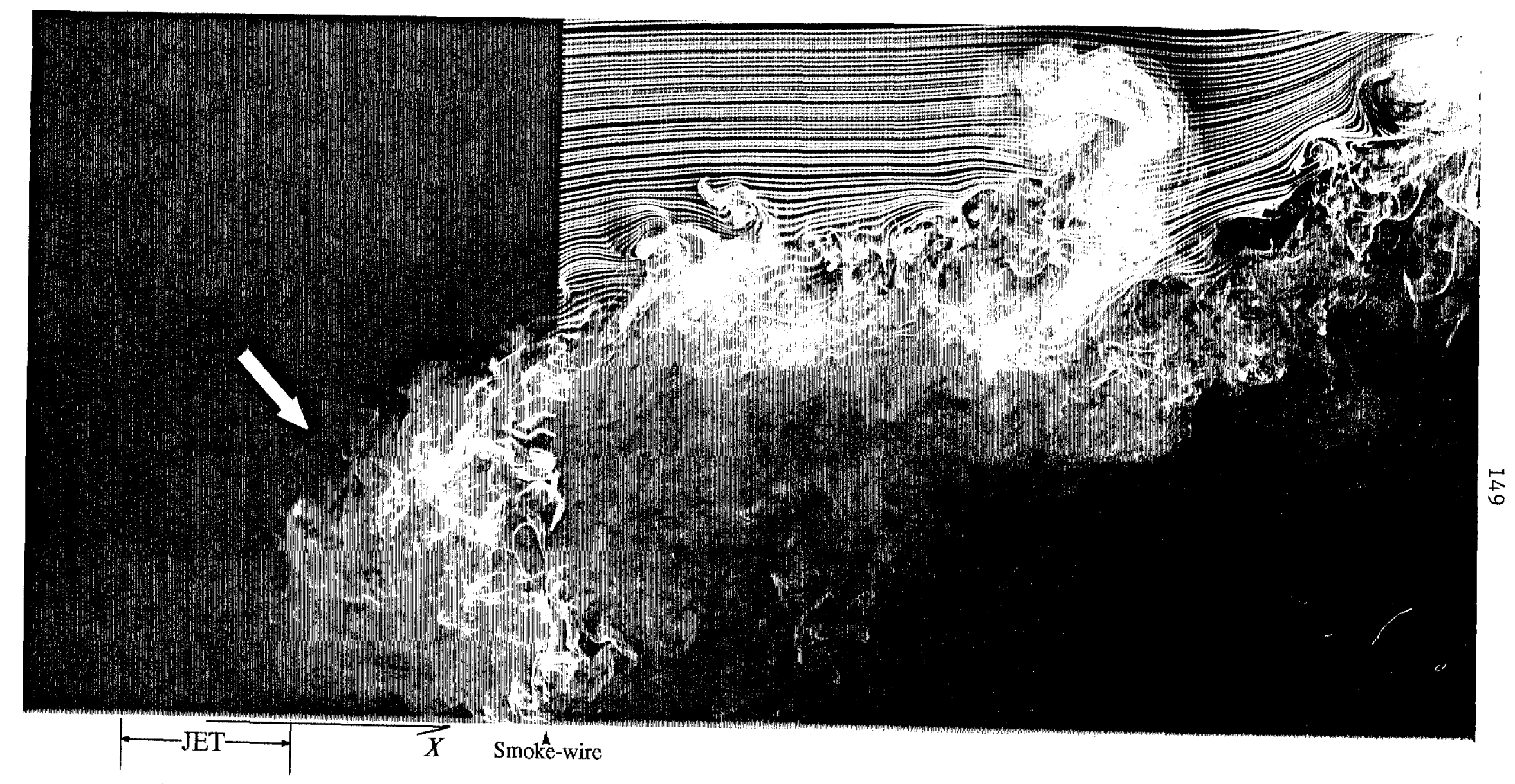

FIGURE 3.14a: $V R=2$.

FIGURE 3.14: Reverse flow region at the near wall, very near wake for (a) $V R=2$, (d) $V R=8$ and (e) $V R=10$. No reverse flow is observed for (b) $V R=4$ and (c) $V R=6 . \quad R e_{c f}=3800$. $X_{s w} / D_{j}=2 . Y_{s w} / D_{j}=0$. 


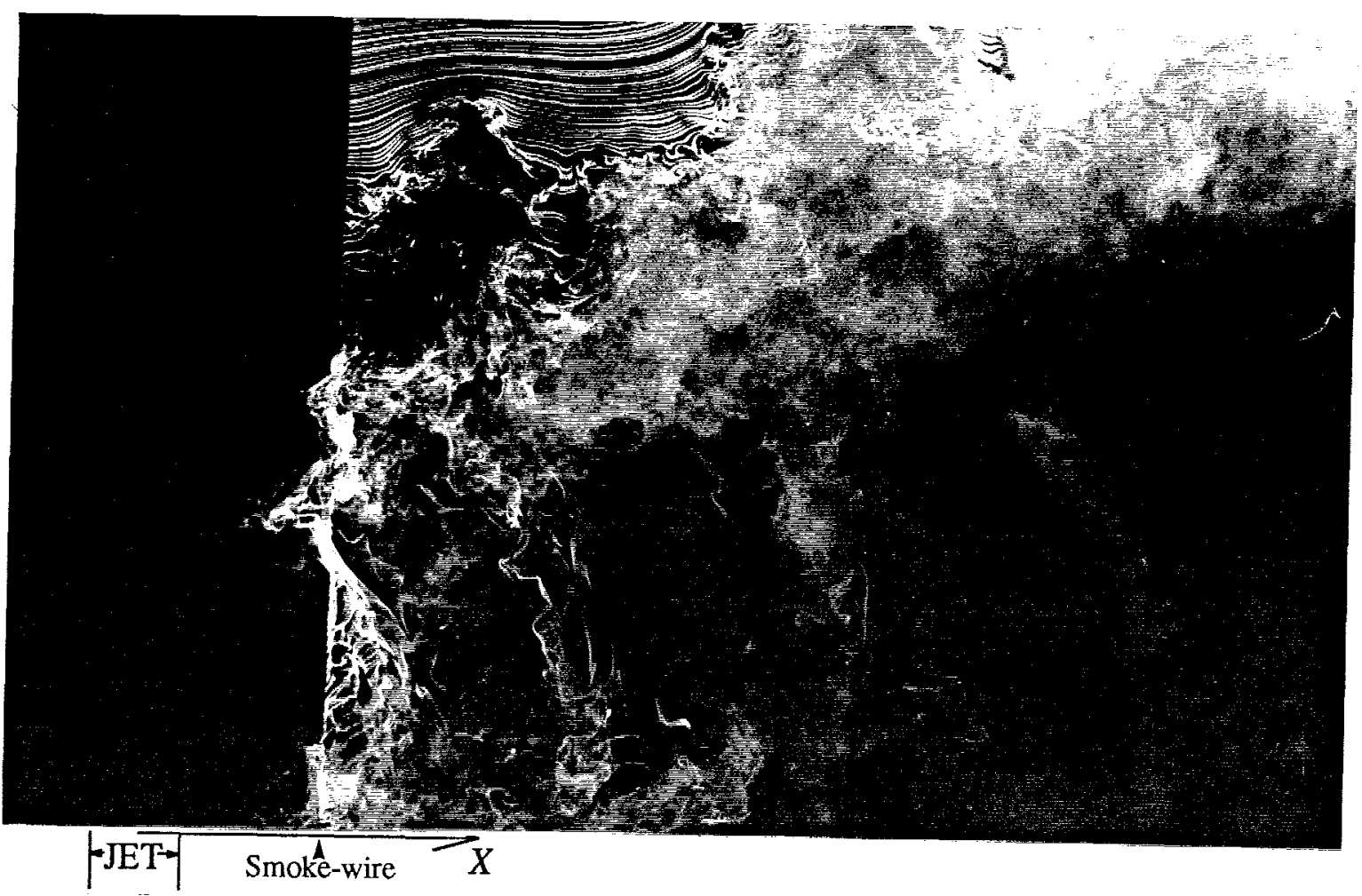

FIGURE 3.14b: $V R=4$.

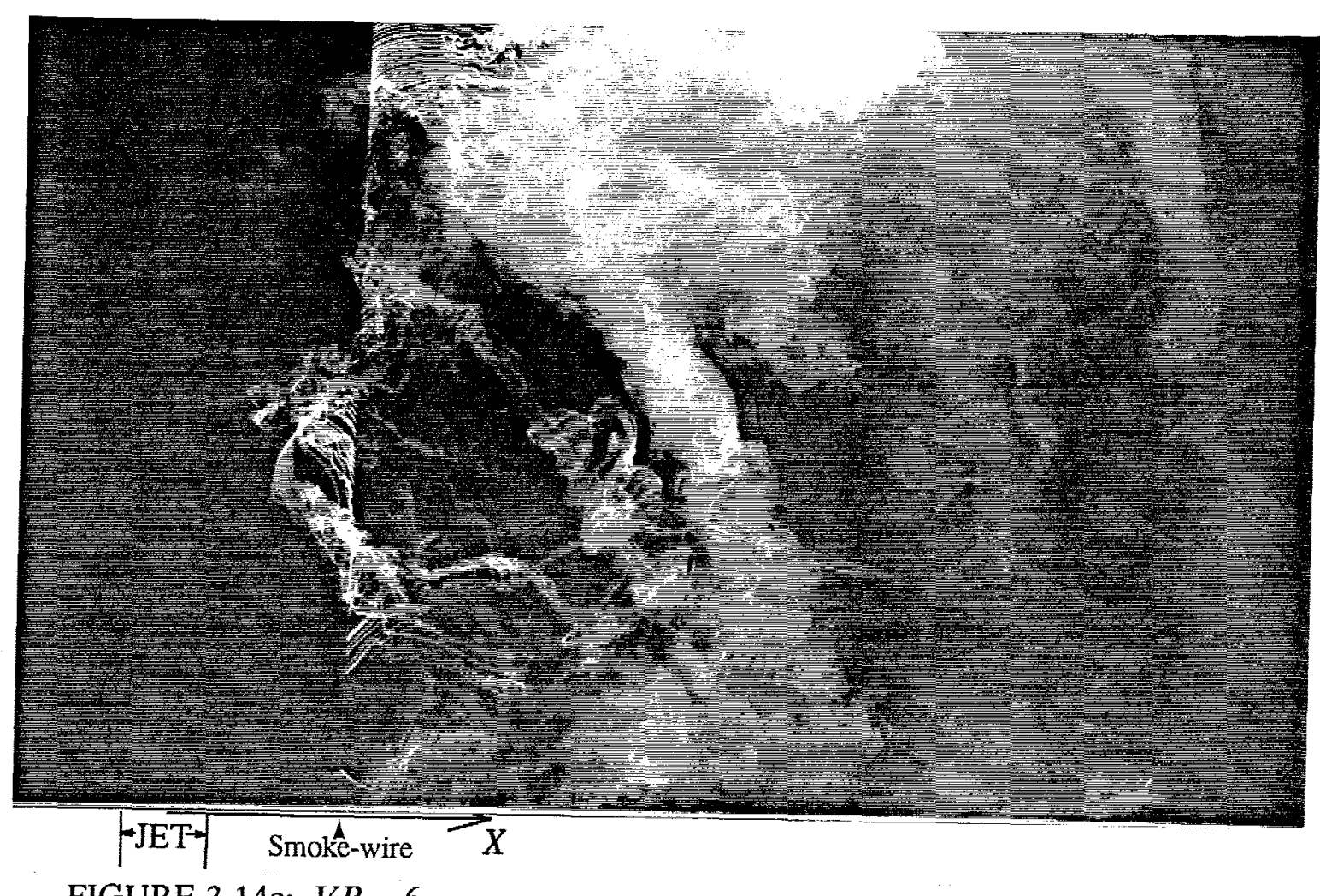

FIGURE 3.14c: $V R=6$. 


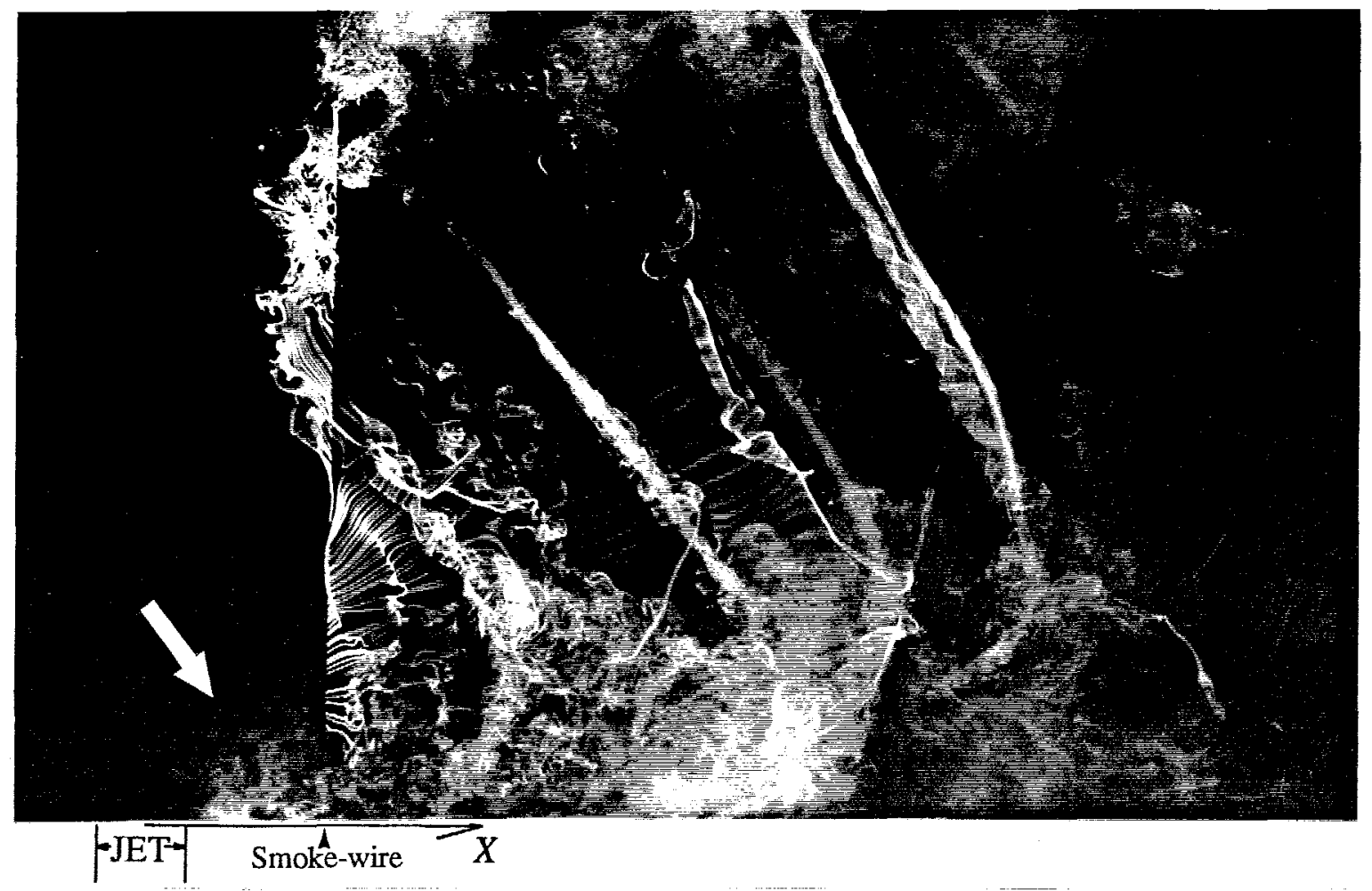

FIGURE 3.14d: $\quad V R=8$.

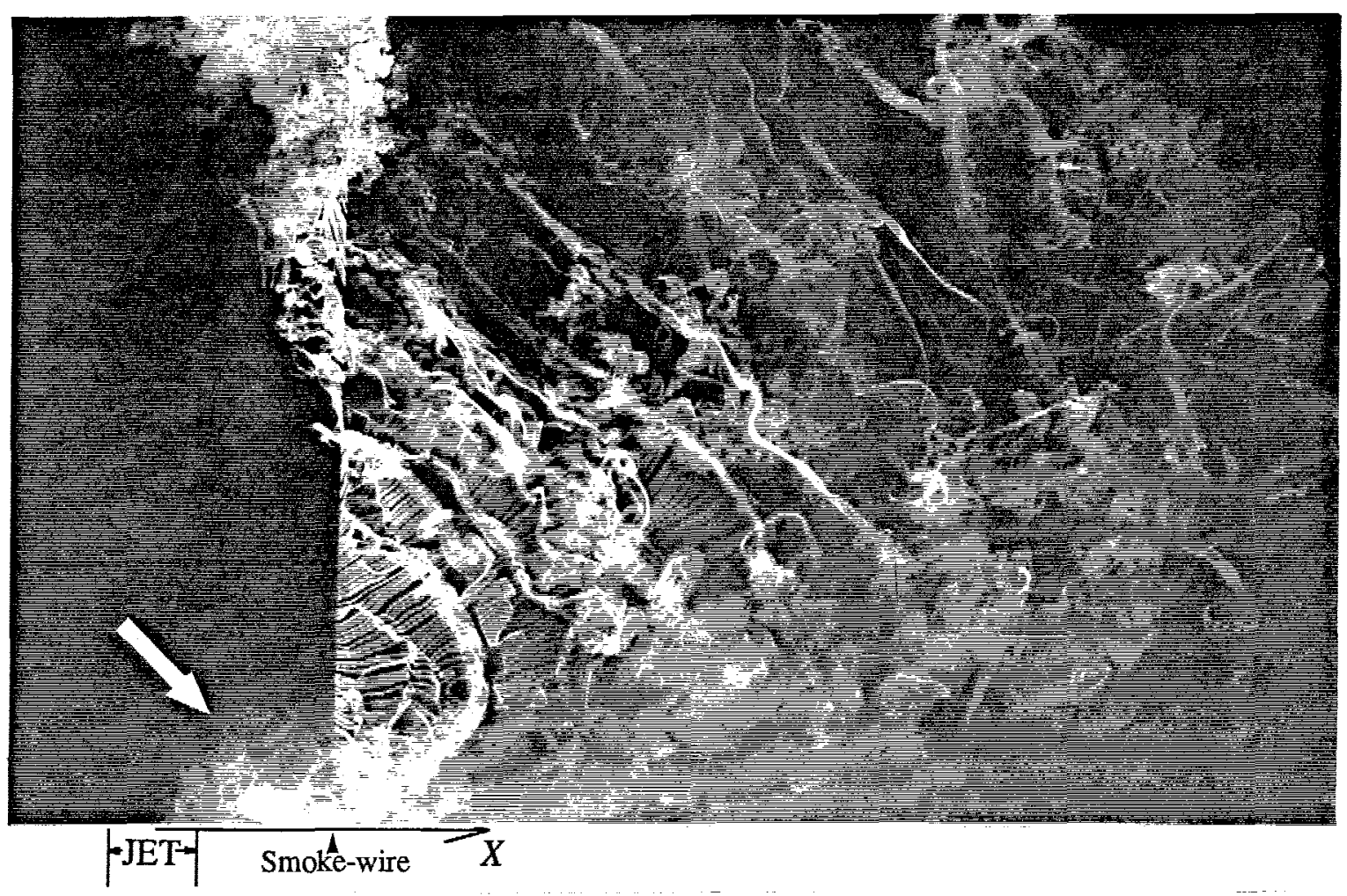

FIGURE 3.14e: $V R=10$. 

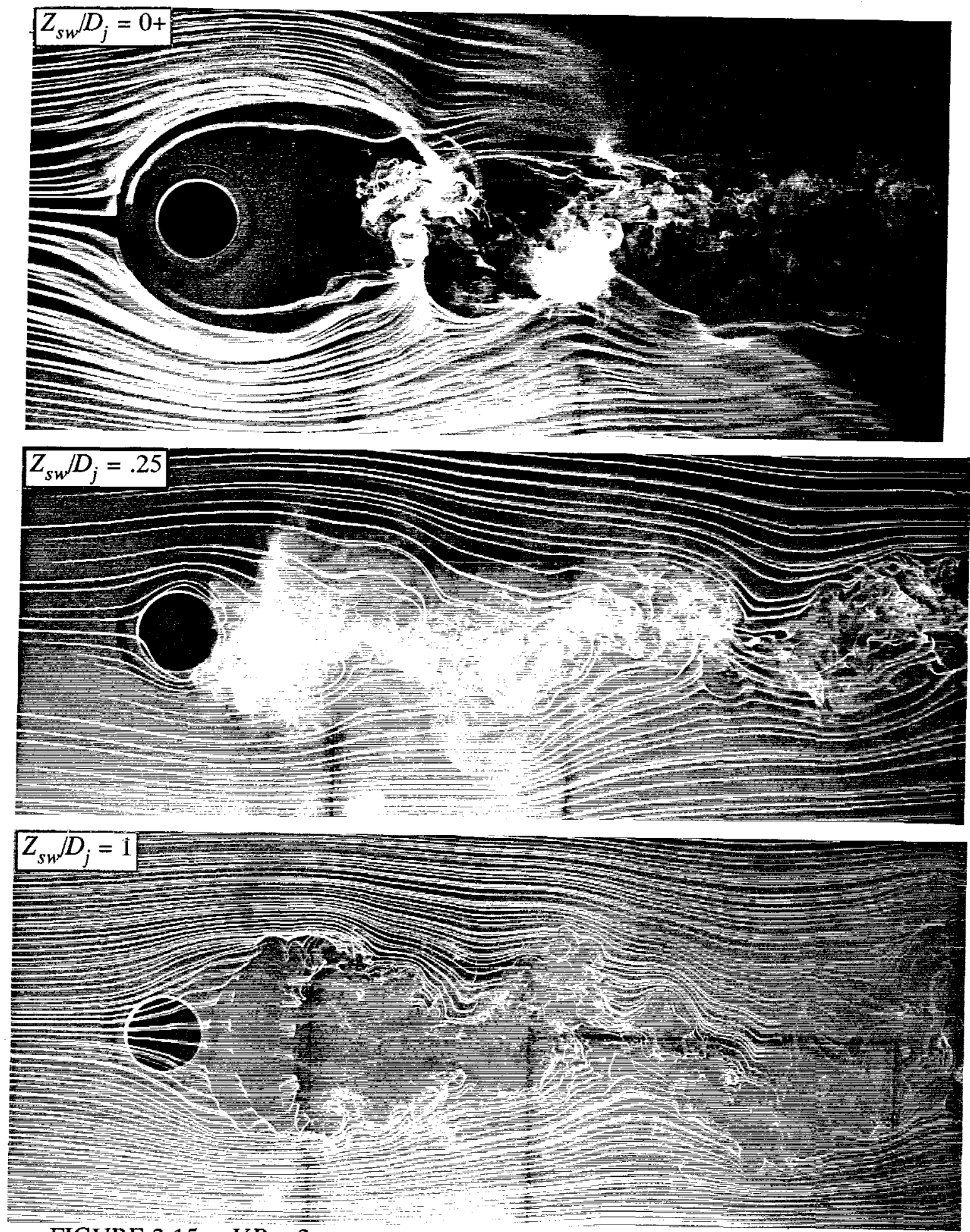

FIGURE 3.15a: $V R=2$.

FIGURE 3.15: Cross sectional views of the wake at various distances from the crossflow wall. Jet issues at the viewer. (a) $V R=2$, (b) $V R=4$, (c) $V R=6$, (d) $V R=8$, (e) $V R=10 . R e_{c f}=3800$. 

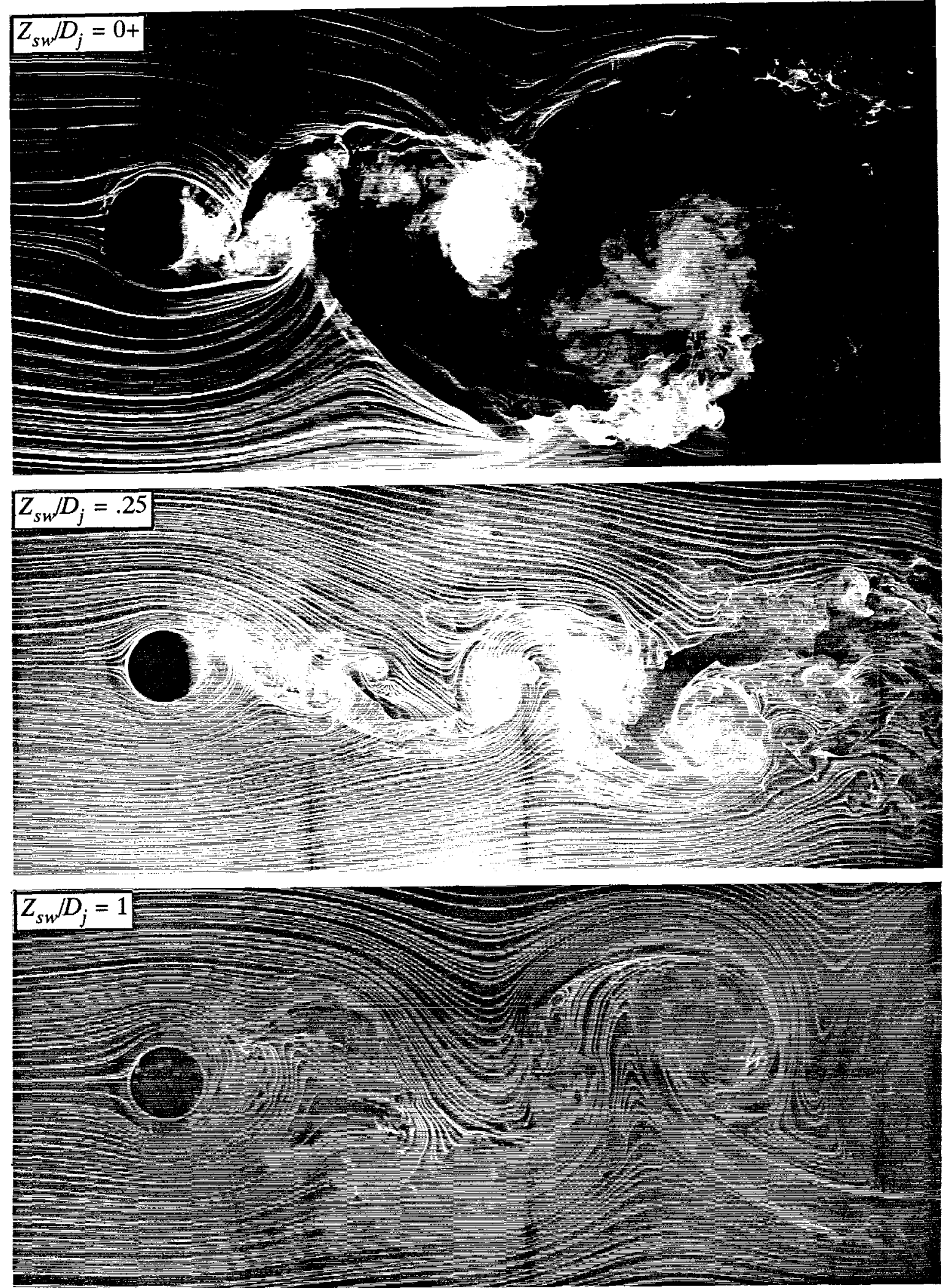

FIGURE 3.15b: $V R=4$. 

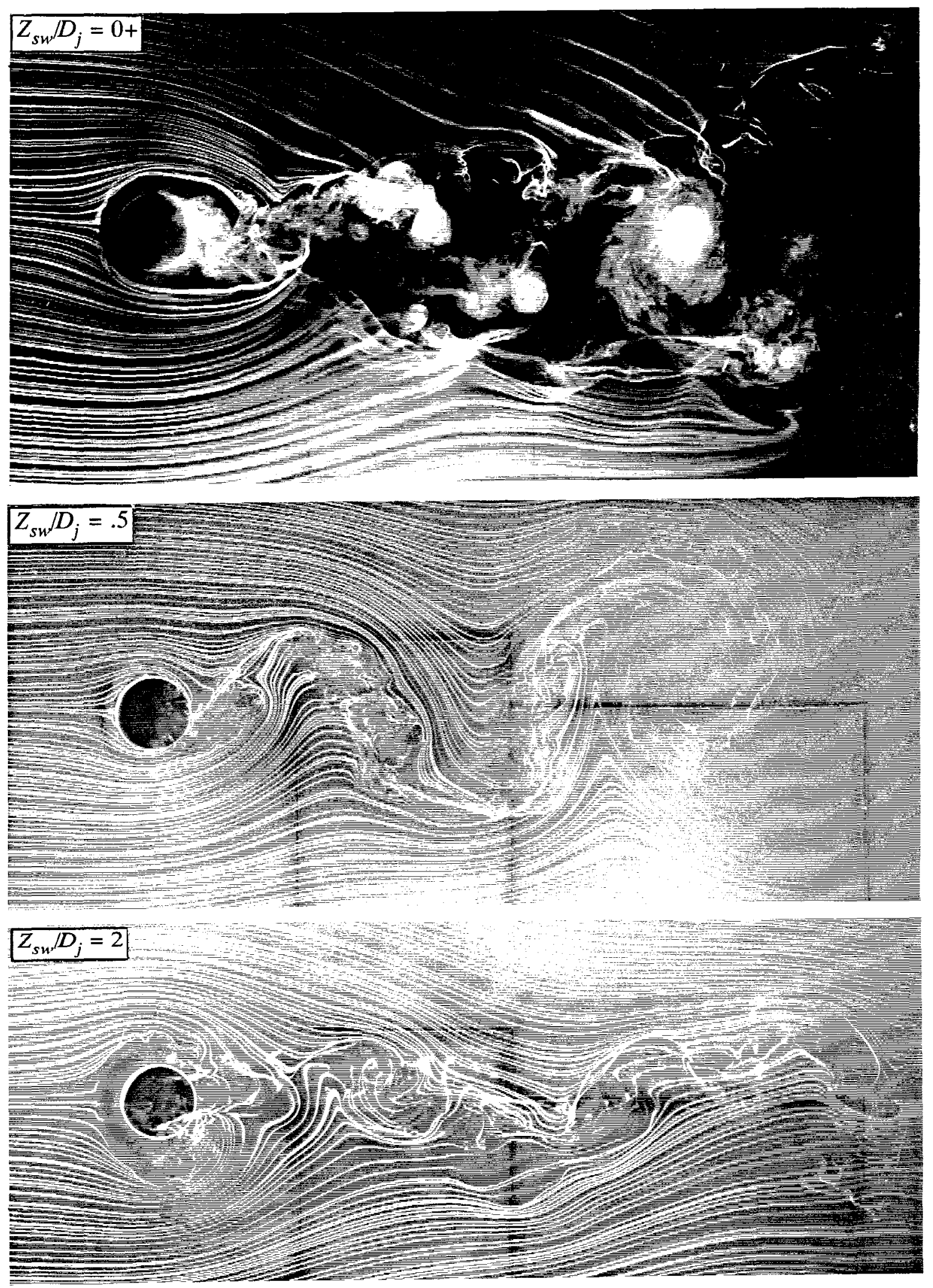

FIGURE 3.15c: $V R=6$. 

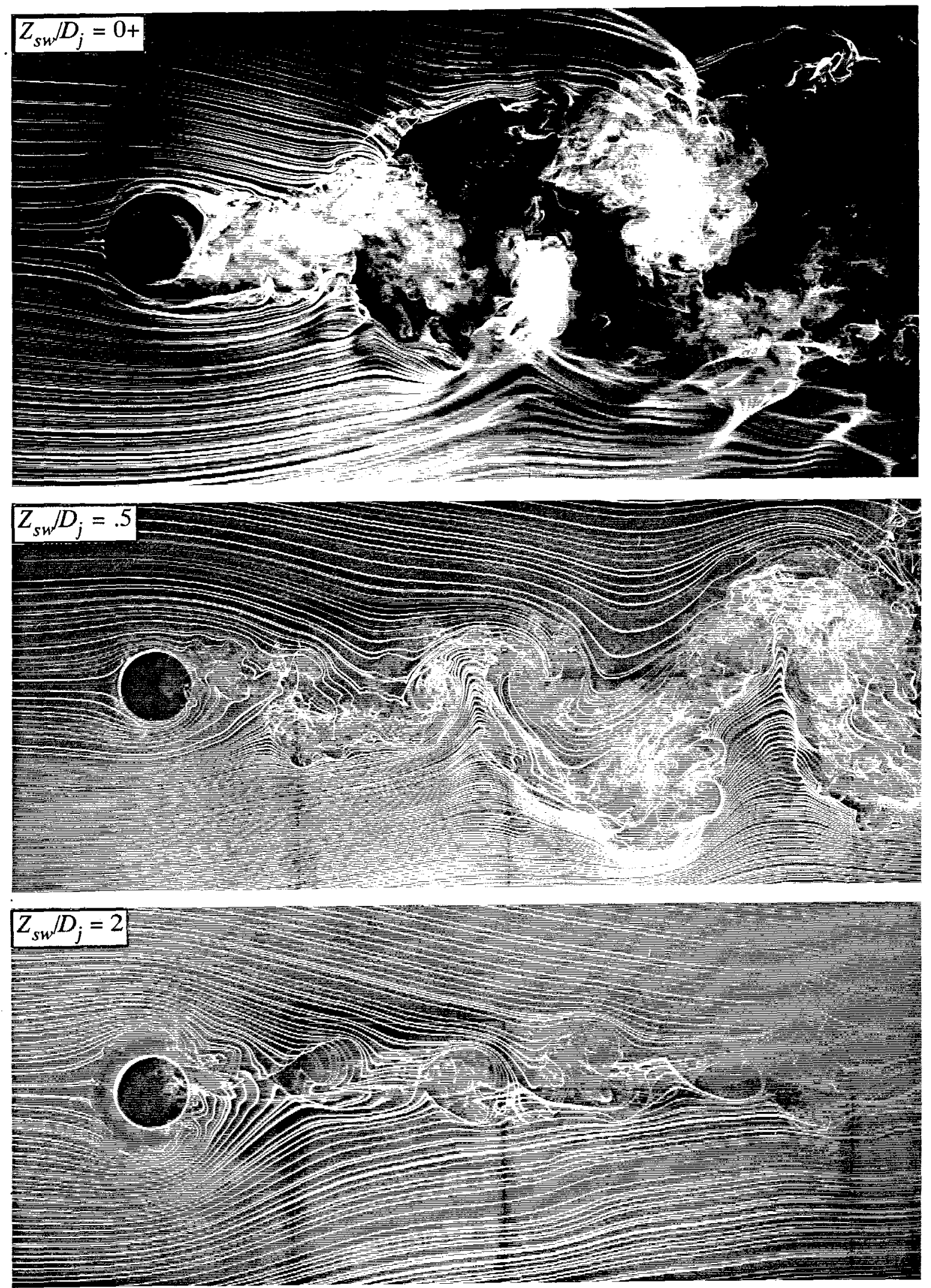

FIGURE 3.15d: $V R=8$. 

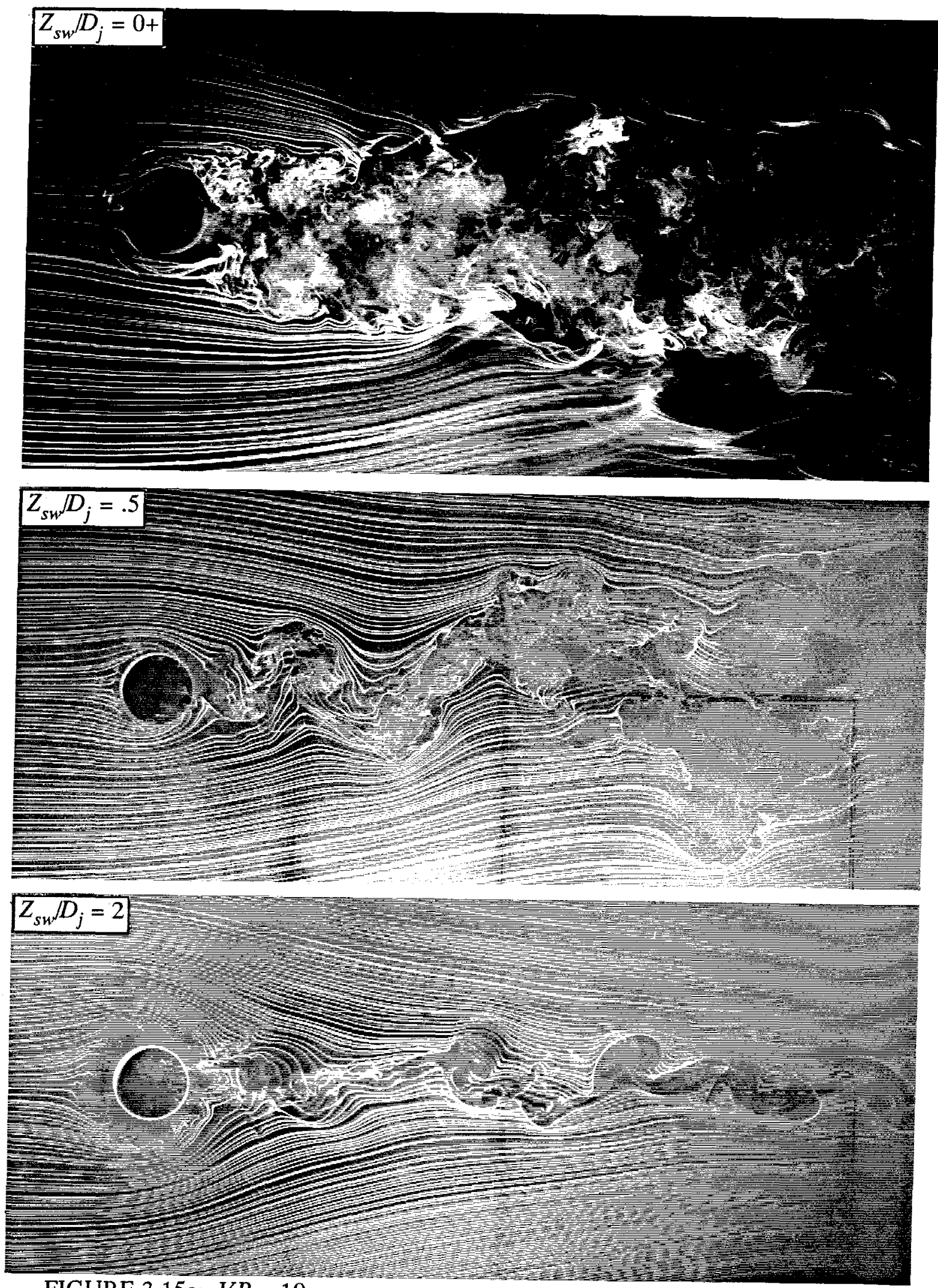

FIGURE 3.15e: $V R=10$. 


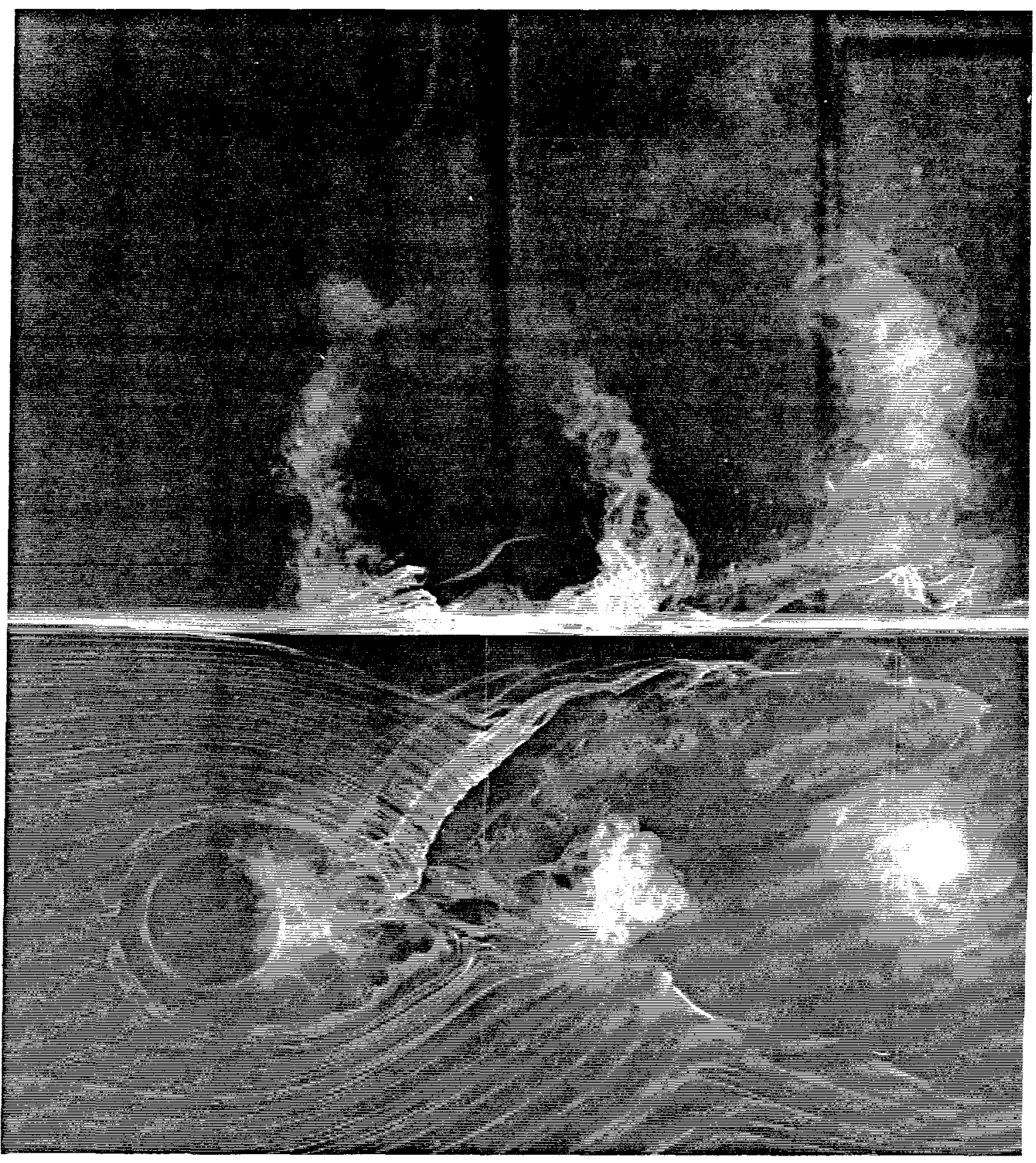

FIGURE 3.16: Simultaneous cross sectional and side view of the wake vortices. $V R=4$. $R e_{c f}=3800 . Z_{s w} D D_{j}=0+$. 


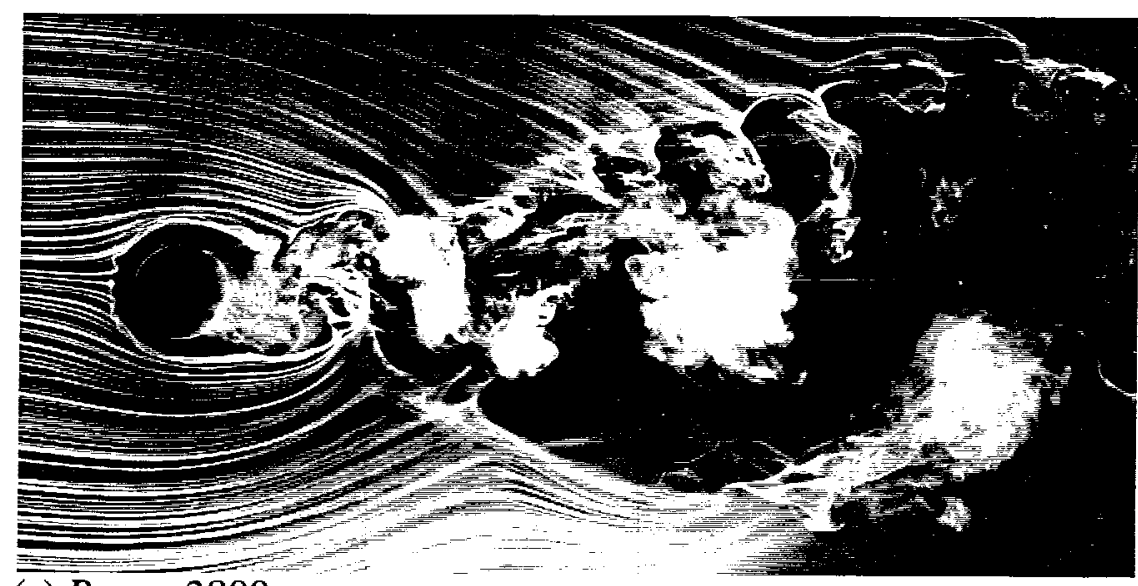

(a) $R e_{c f}=3800$.

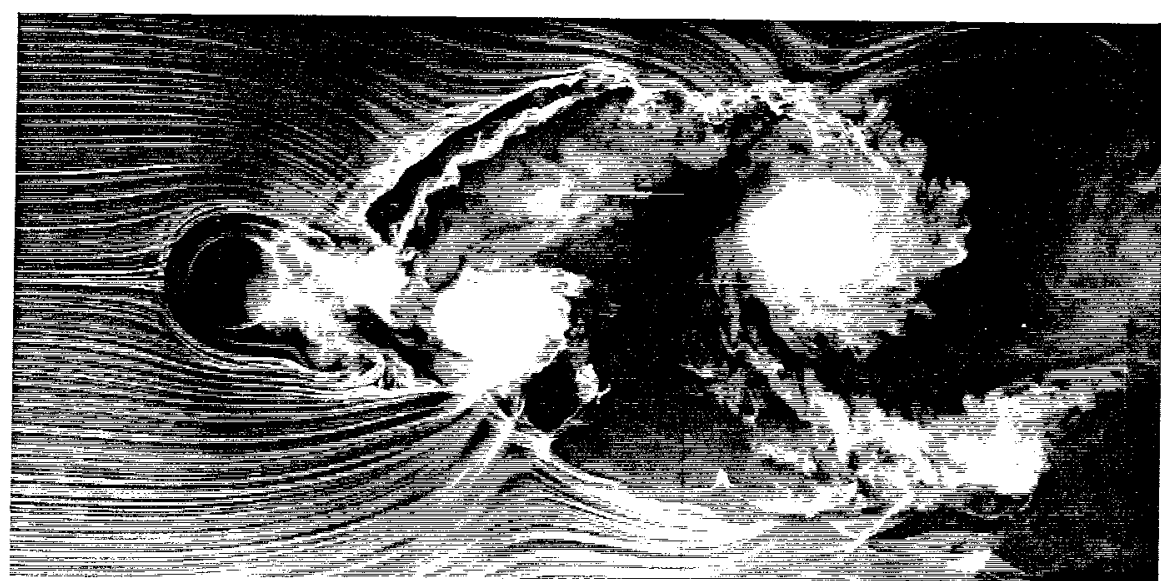

(b) $R e_{c f}=7600$.

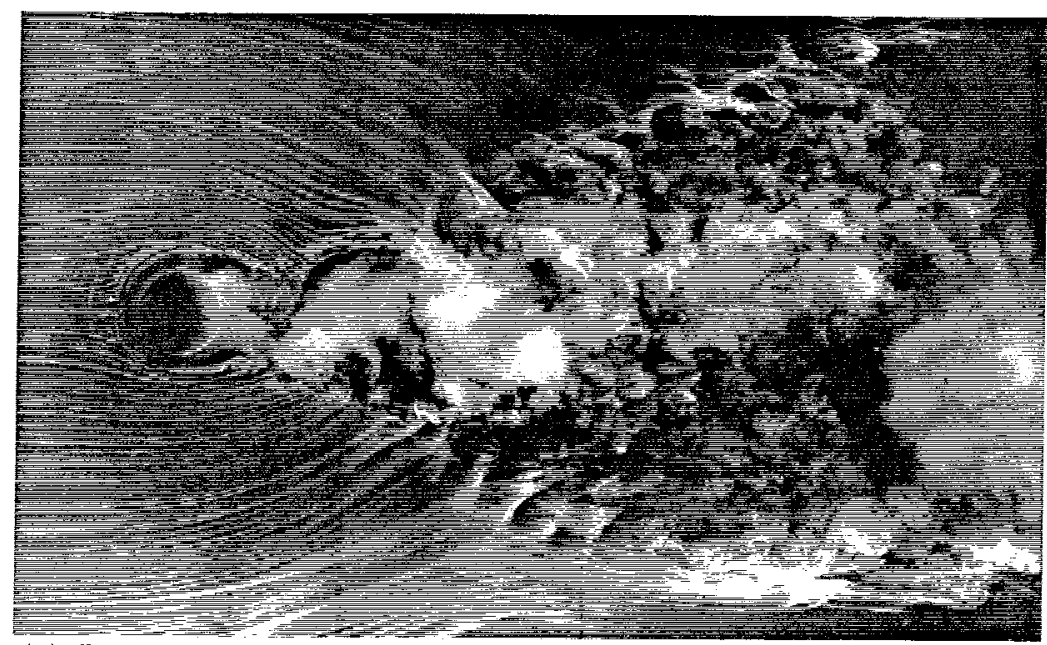

(c) $R e_{c f}=11400$.

FIGURE 3.17: Effects of crossflow Reynolds number on near wall wake flow.

(a) $R e_{c f}=3800$, (b) $R e_{c f}=7600$, (c) $R e_{c f}=11400$.

$V R=6 . Z_{s w} D_{j}=0+$. 


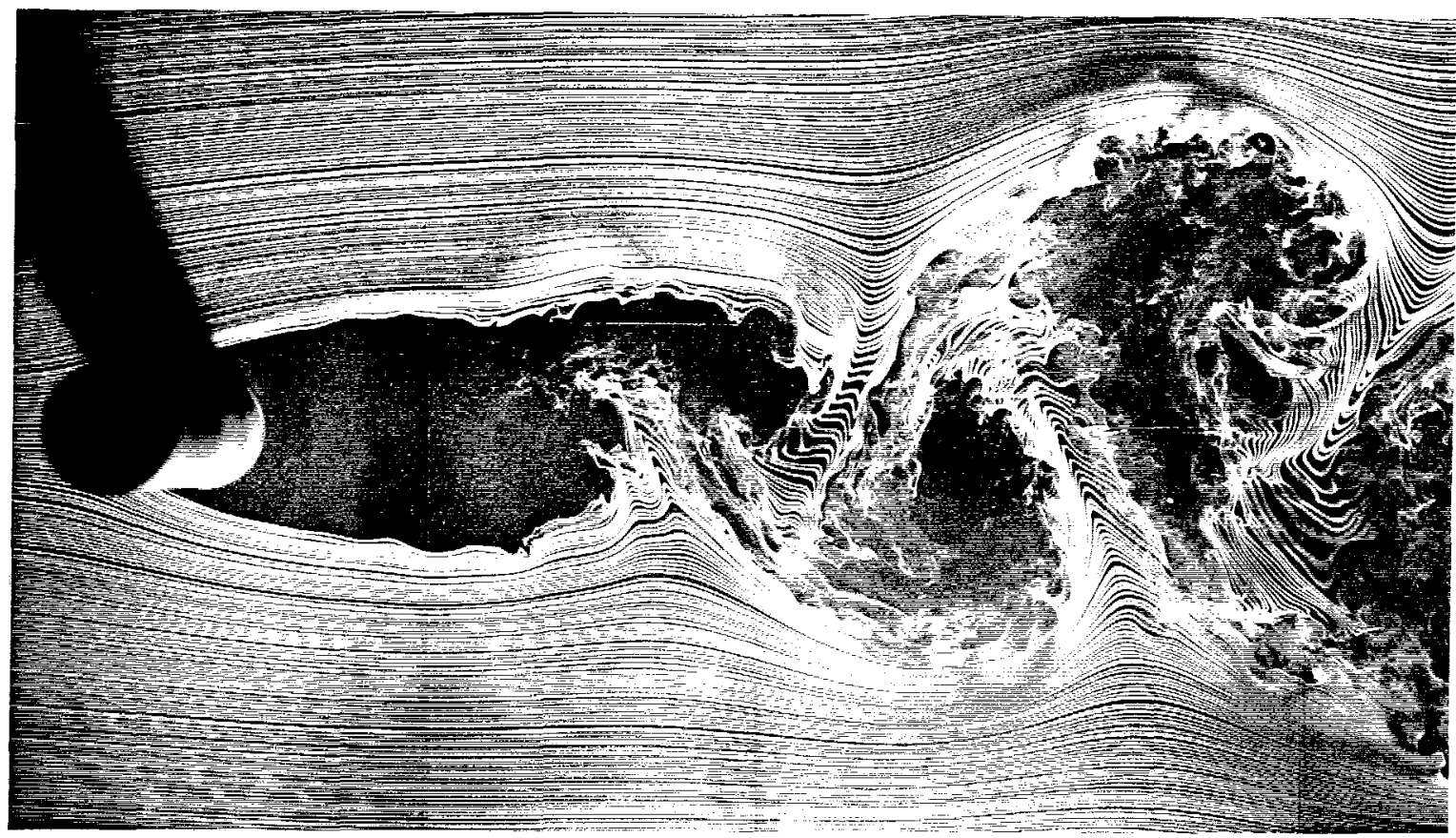

(a) $A R=6$ cylinder.

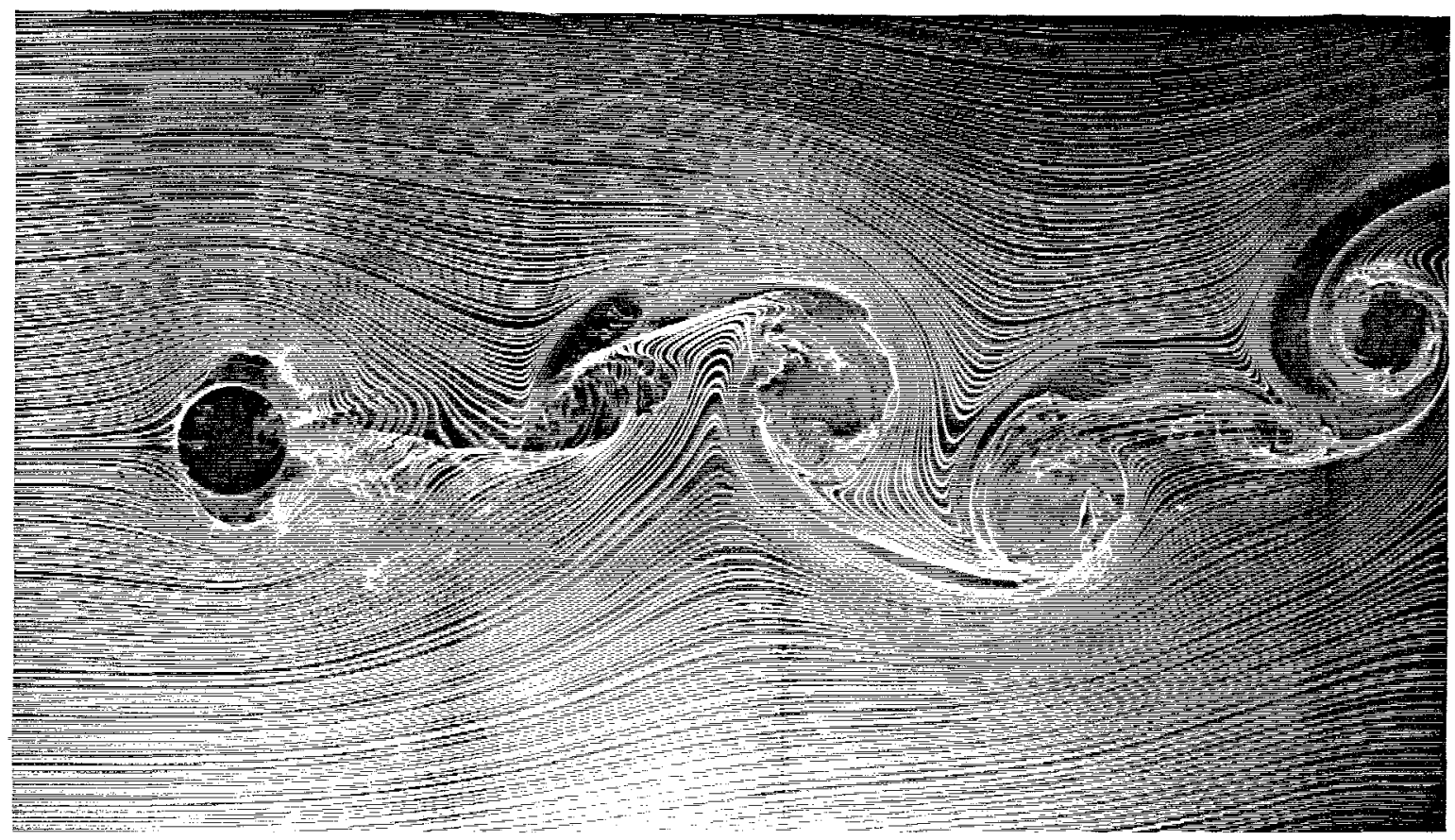

(b) $V R=4$ transverse jet.

FIGURE 5.1: Comparison of a circular cylinder wake with the wake of a transverse jet. (a) $A R=6$ cylinder, (b) $V R=4$ transverse jet. $R e_{c f}=7600$. $Z_{s w} / D_{c}=Z_{s w} / D_{j}=1$. 


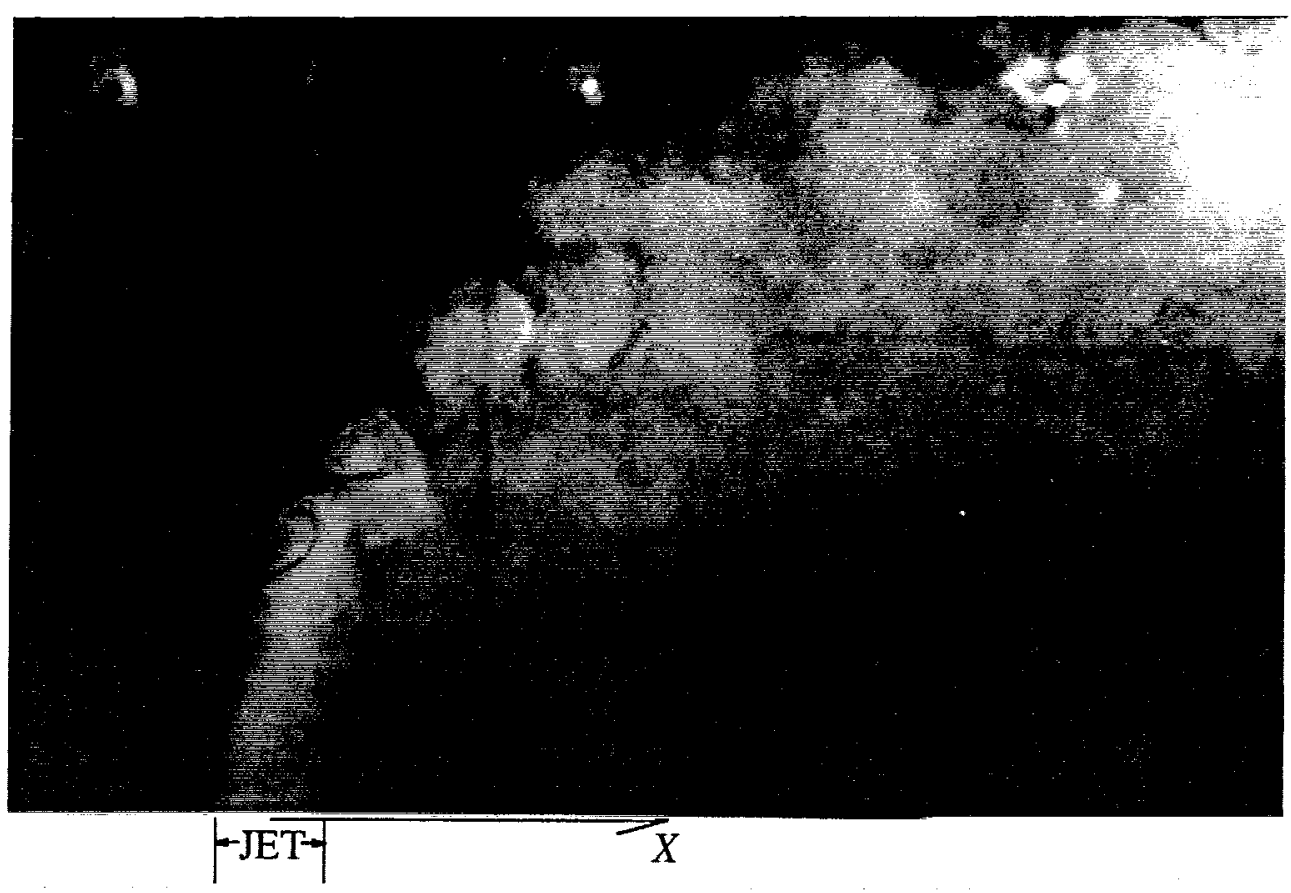

(a) Smoke tagging jet fluid.

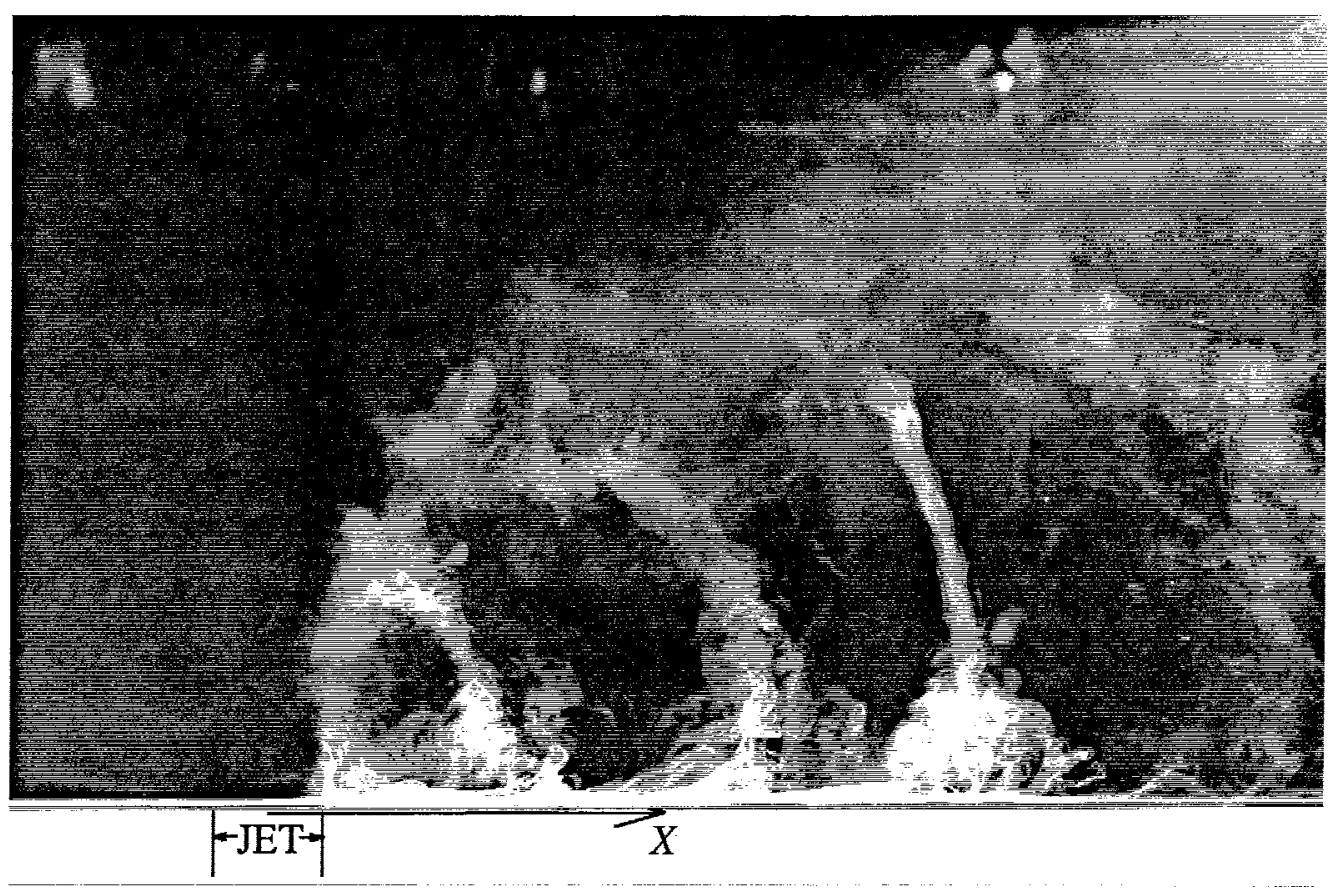

(b) Smoke tagging crossflow boundary layer fluid.

FIGURE 5.2: Using smoke as a vorticity marker. (a) Jet is tagged with smoke, (b) Smoke is initially in the crossflow boundary layer. The view in (b) is identical to that in (a). $V R=4 . R e_{c f}=3800$. 


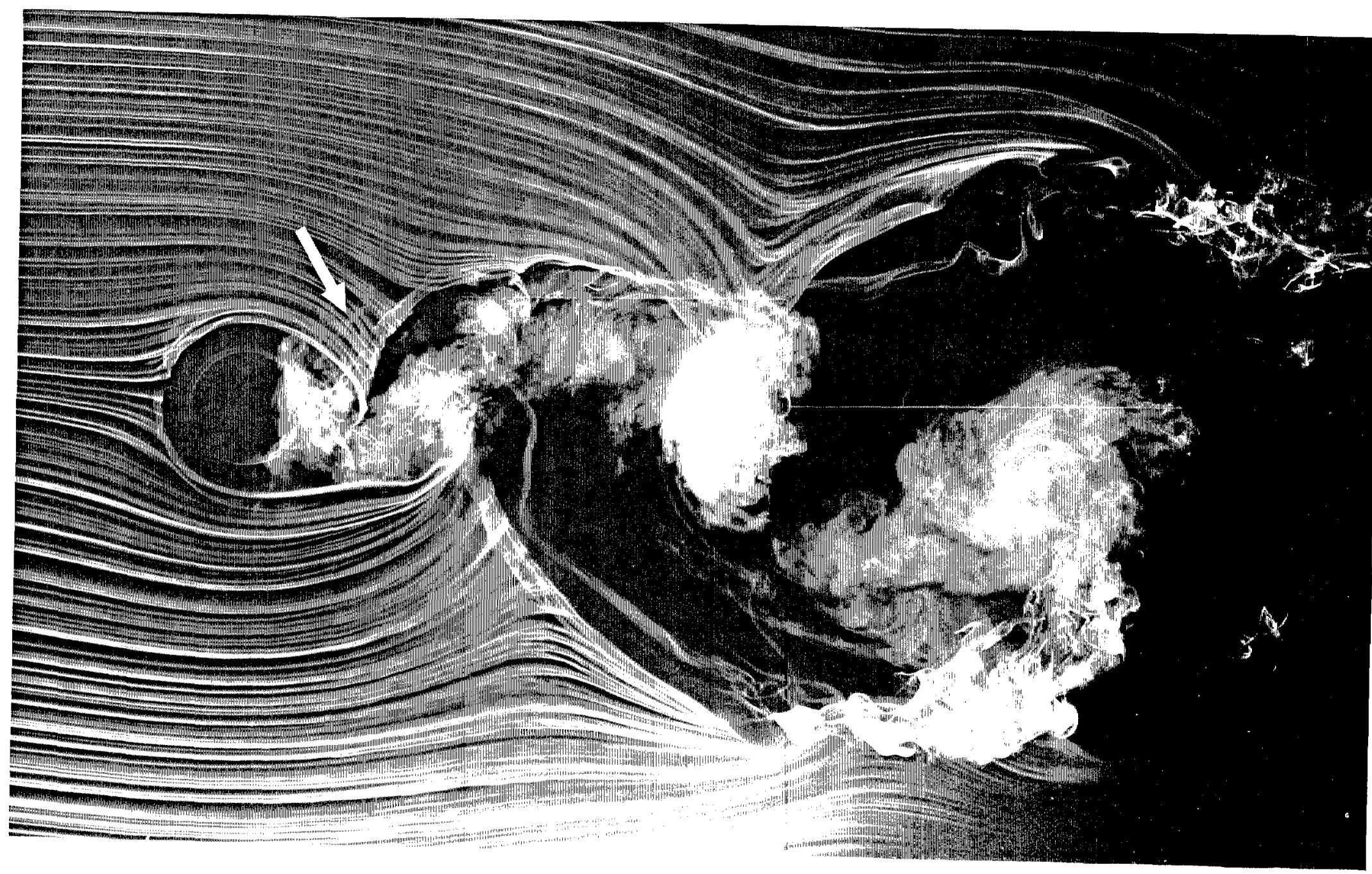

FIGURE 5.3: Separation event and the accompanying near wall flow. Smoke-wire flow visualization $\left(Z_{s w} / D_{j}=0+\right)$. Arrow indicates a separation event $V R=4 . R e_{c f}=3800$. 


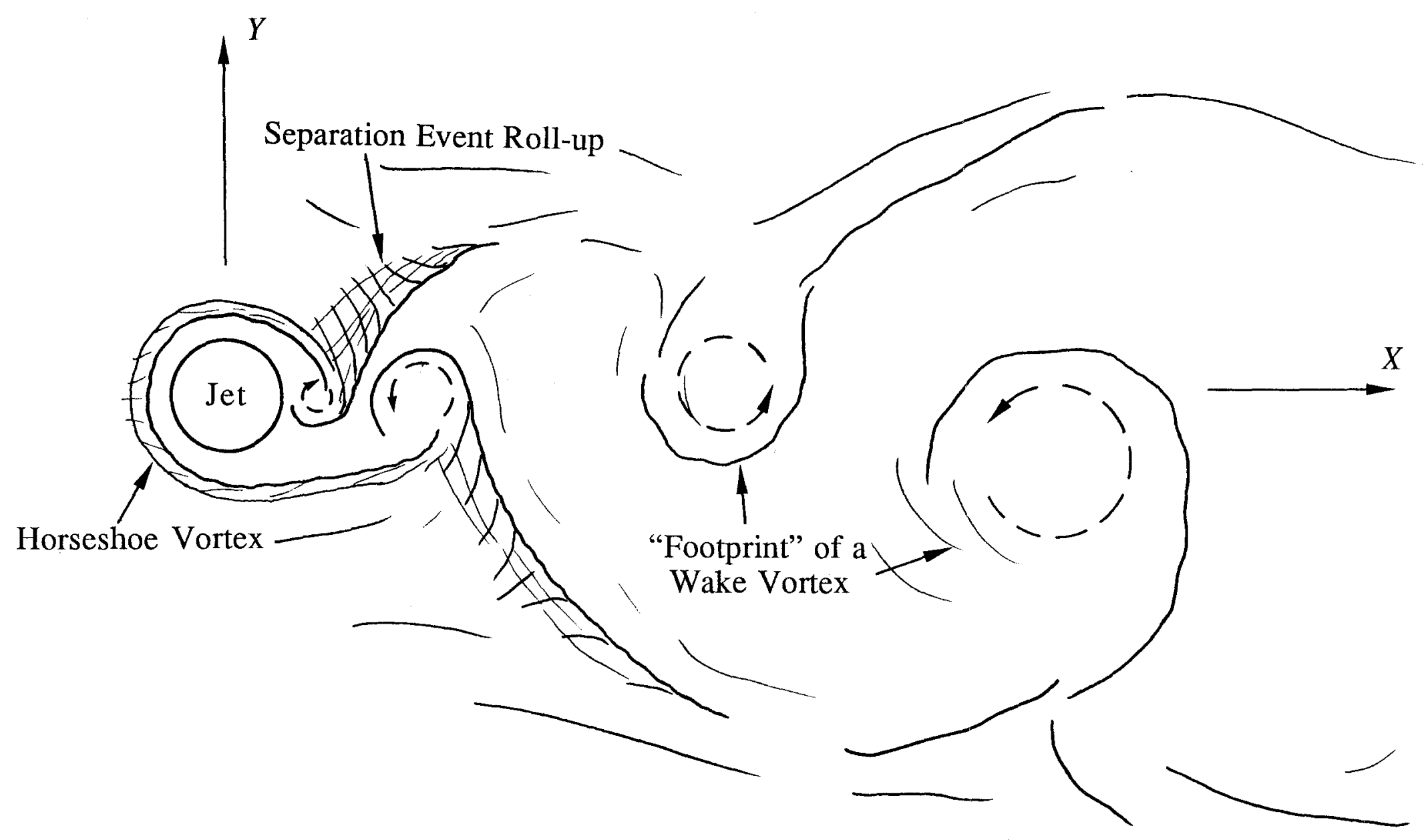

FIGURE 5.4: Sketch highlighting the structures seen in the photograph of figure 5.3. 


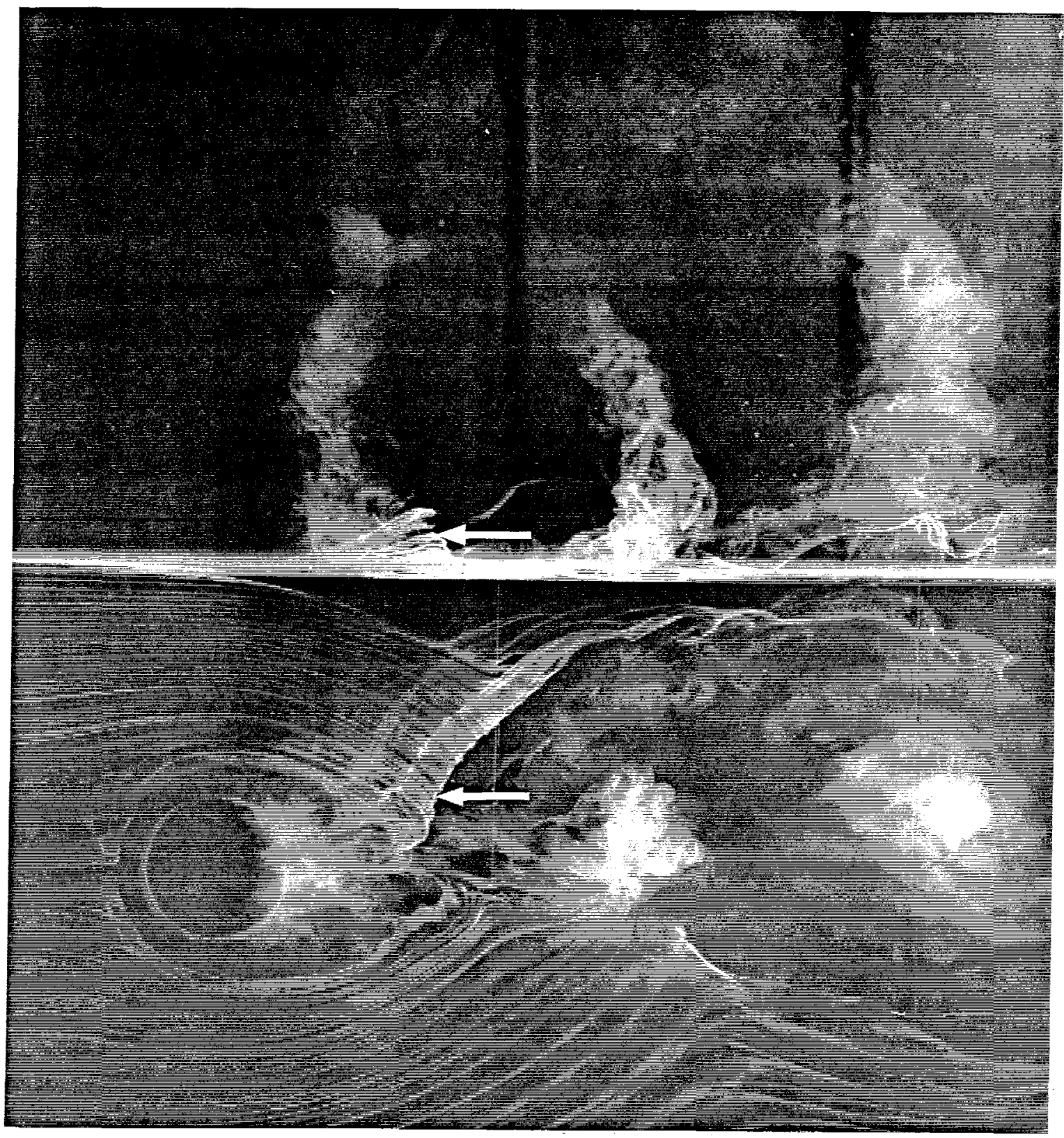

FIGURE 5.5: Simultaneous cross sectional view (bottom) and side view (top) of the wake vortices. The arrows indicate the same separation event in each view. $V R=4 . R e_{c f}=3800 . Z_{s w} / D_{j}=0+$. 


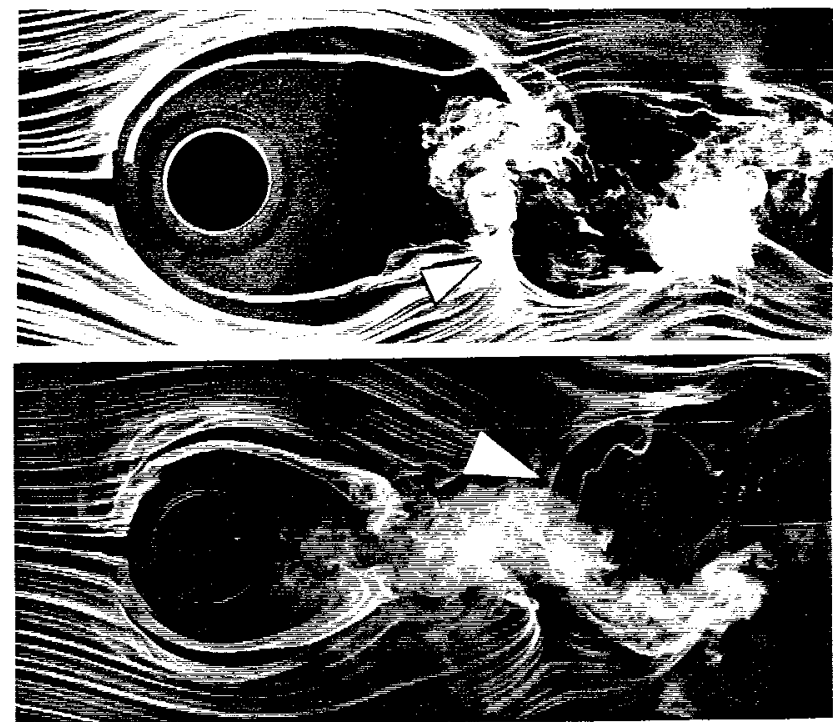

(a) $V R=2$.

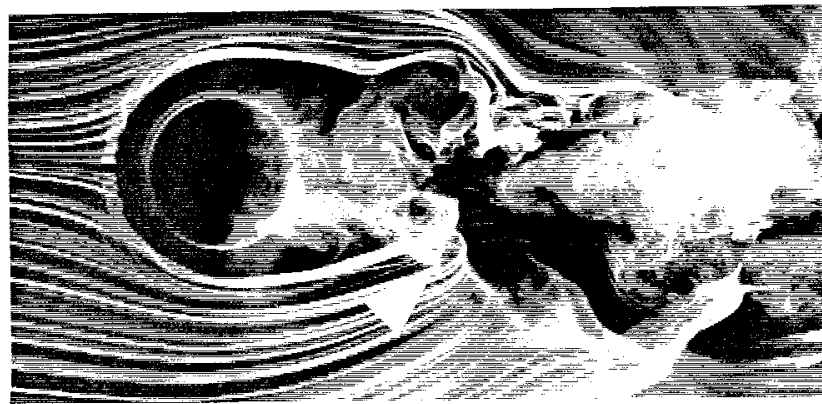

(c) $V R=6$

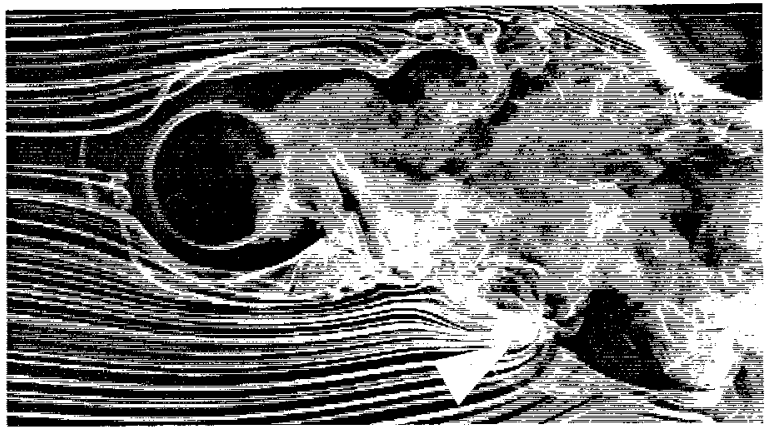

(b) $V R=4$.

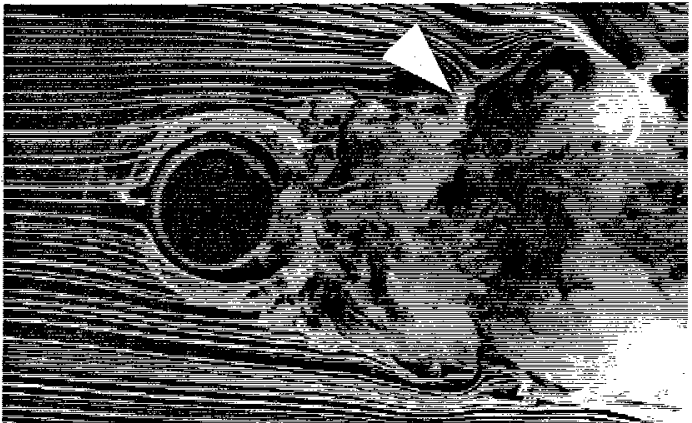

(e) $V R=10$

FIGURE 5.6: Typical near wall, very near wakes showing crossflow boundary layer separations. (a) $V R=2$, (b) $V R=4$, (c) $V R=6$, (d) $V R=8$, (e) $V R=10 . R e_{c f}=3800 . Z_{s w} / D_{j}=0+$. 

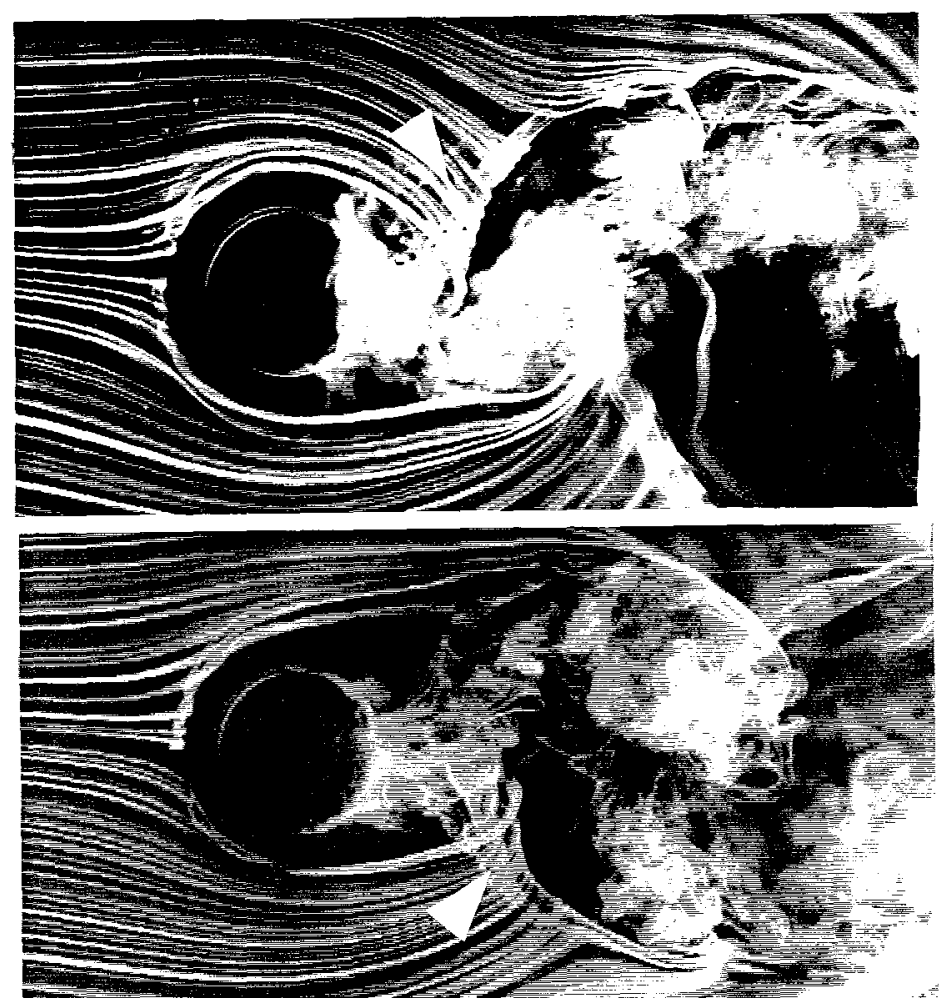

(b) $V R=6$.
(a) $V R=4$.
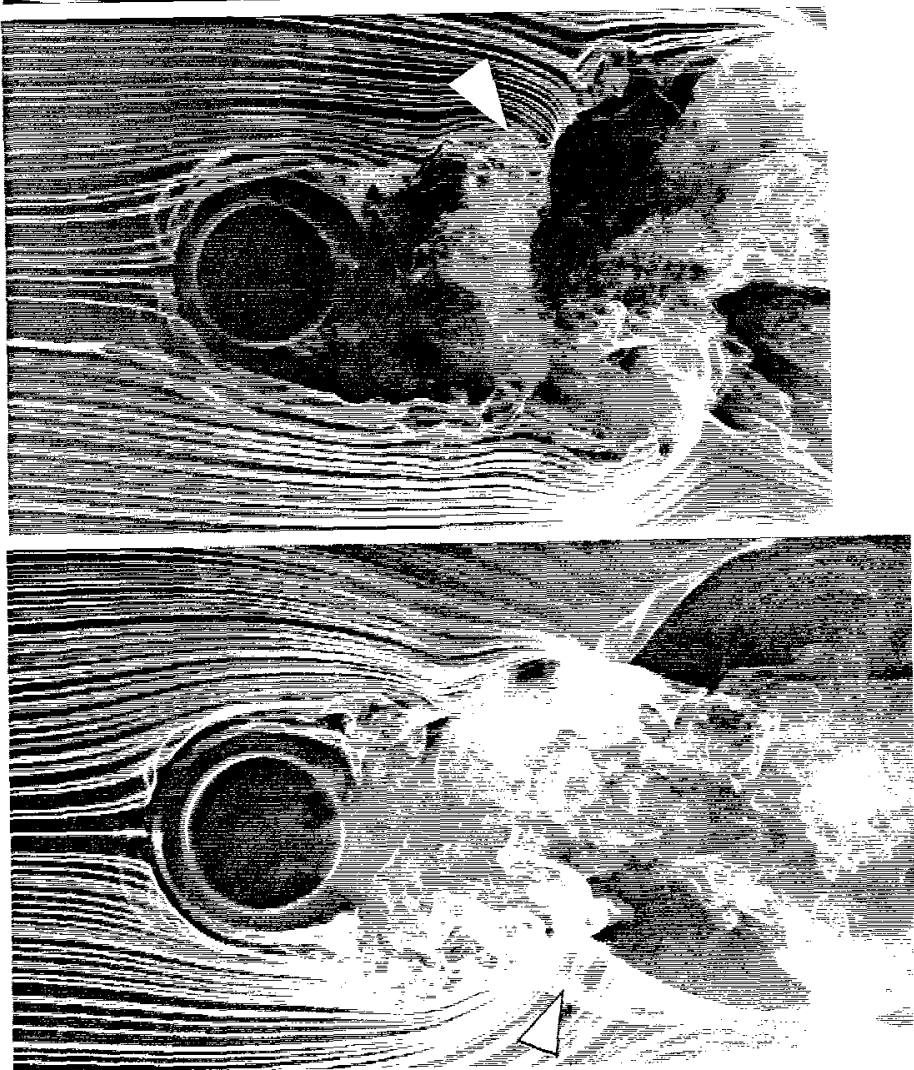

(c) $V R=8$.

(d) $V R=10$.

FIGURE 5.7: Examples where separation events are most clearly evident.

(a) $V R=4$, (b) $V R=6$, (c) $V R=8$, (d) $V R=10$.

$R e_{c f}=3800 . Z_{s w} D_{j}=0+$. 


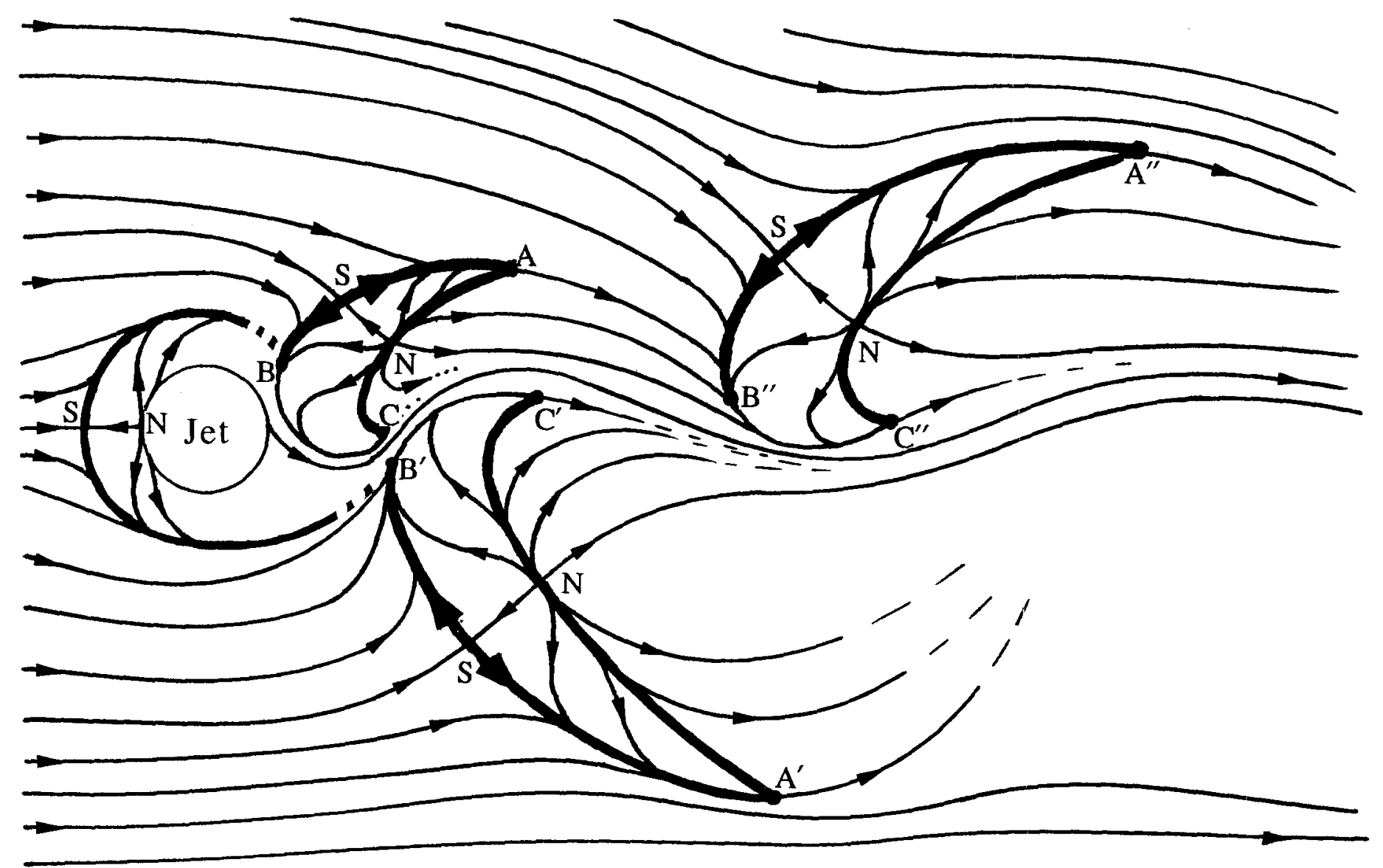

FIGURE 5.8: Instantaneous crossflow wall surface topology/streamline pattern for the flow shown in figure 5.3. Separation lines: $\mathrm{AB}, \mathrm{A}^{\prime} \mathrm{B}^{\prime}, \mathrm{A}^{\prime \prime} \mathrm{B}^{\prime \prime}$. Attachment lines: AC, $\mathrm{A}^{\prime} \mathrm{C}^{\prime}, \mathrm{A}^{\prime \prime} \mathrm{C}^{\prime \prime}$. 


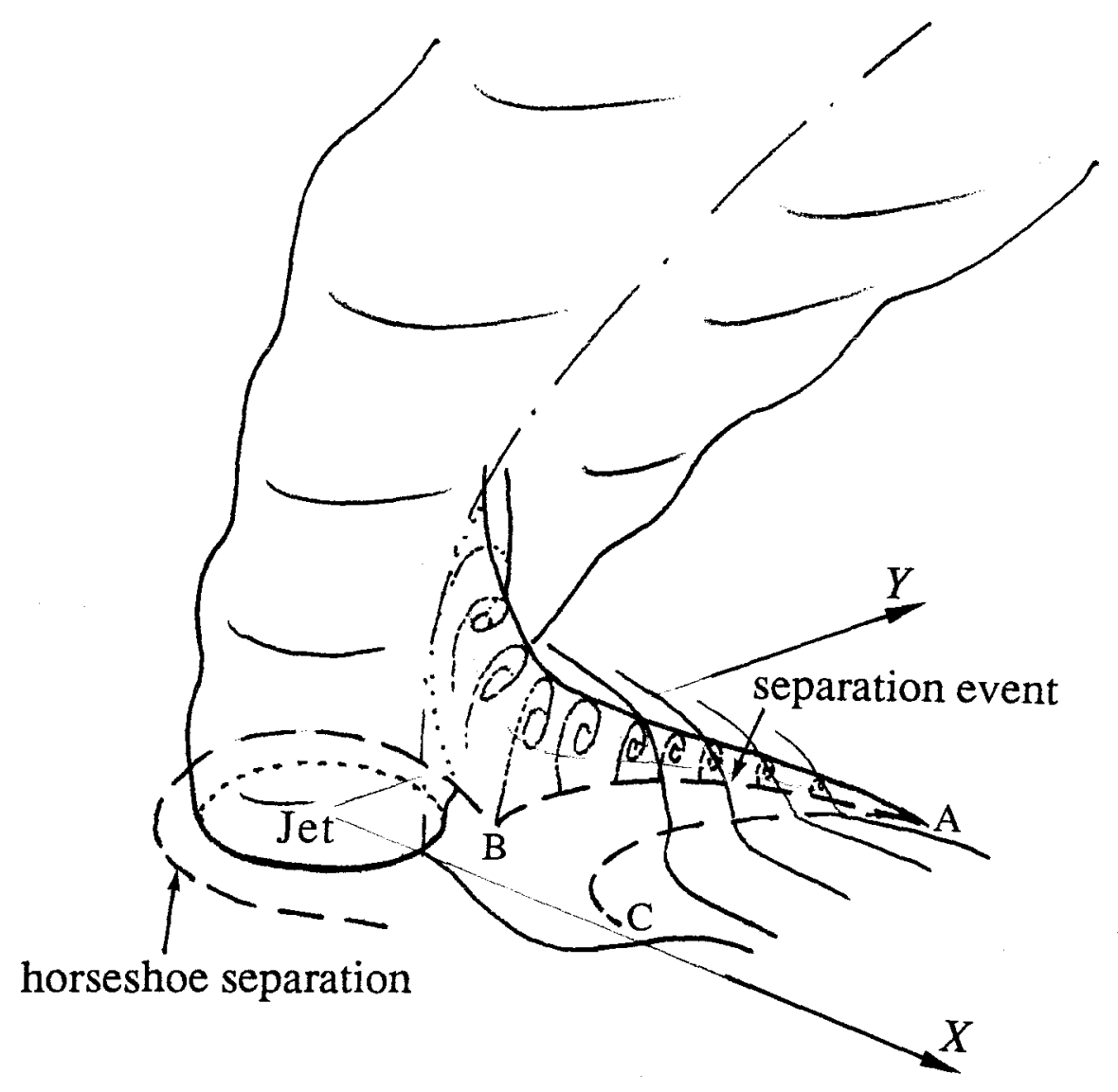

FIGURE 5.9: Flow streamsurface associated with a separation event. 

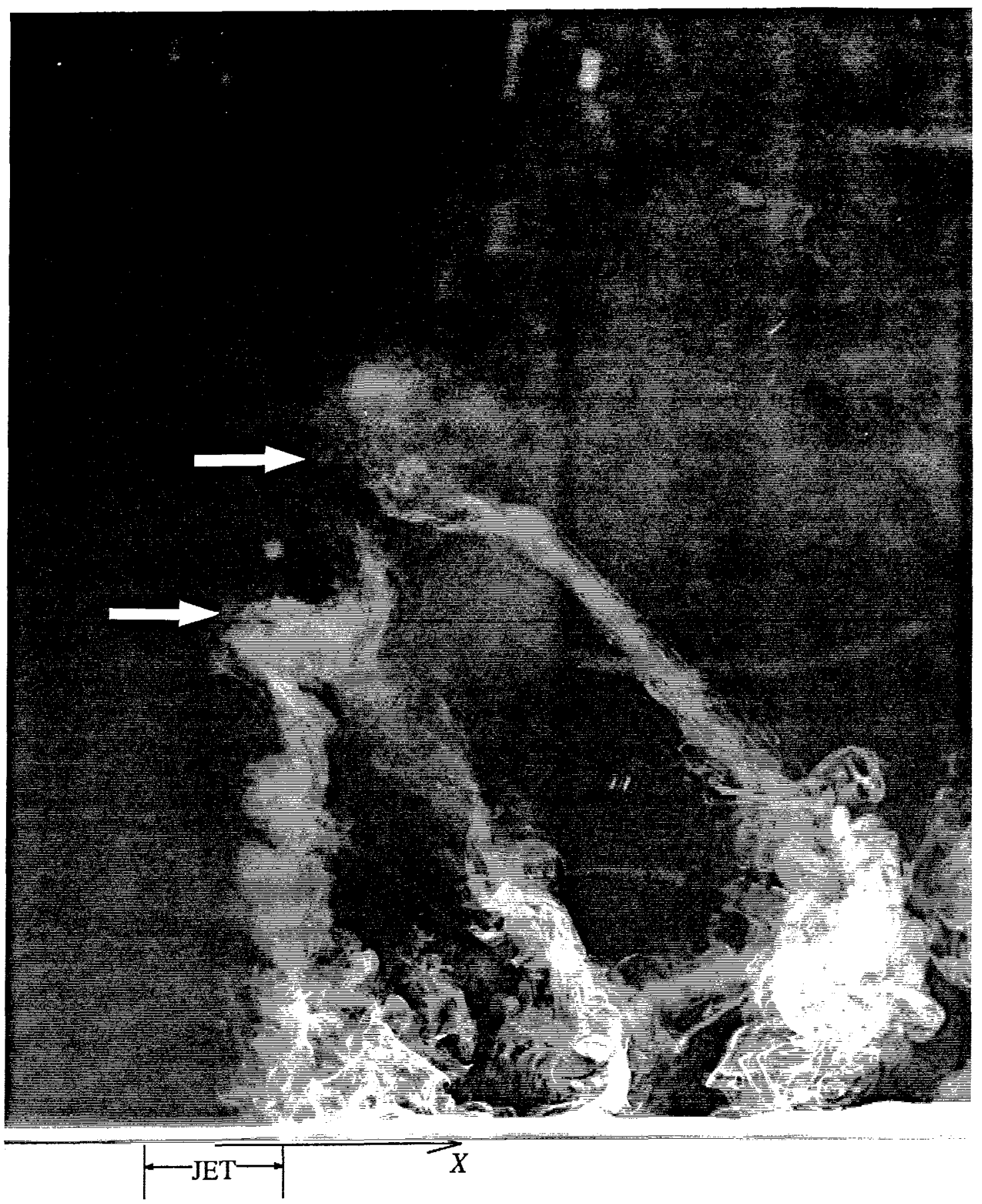

FIGURE 5.10: Two wake vortices attached at the trailing edge of the jet. $V R=$ 5.8. $R e_{c f}=3800 . Z_{s w} / D_{j}=0+$. 

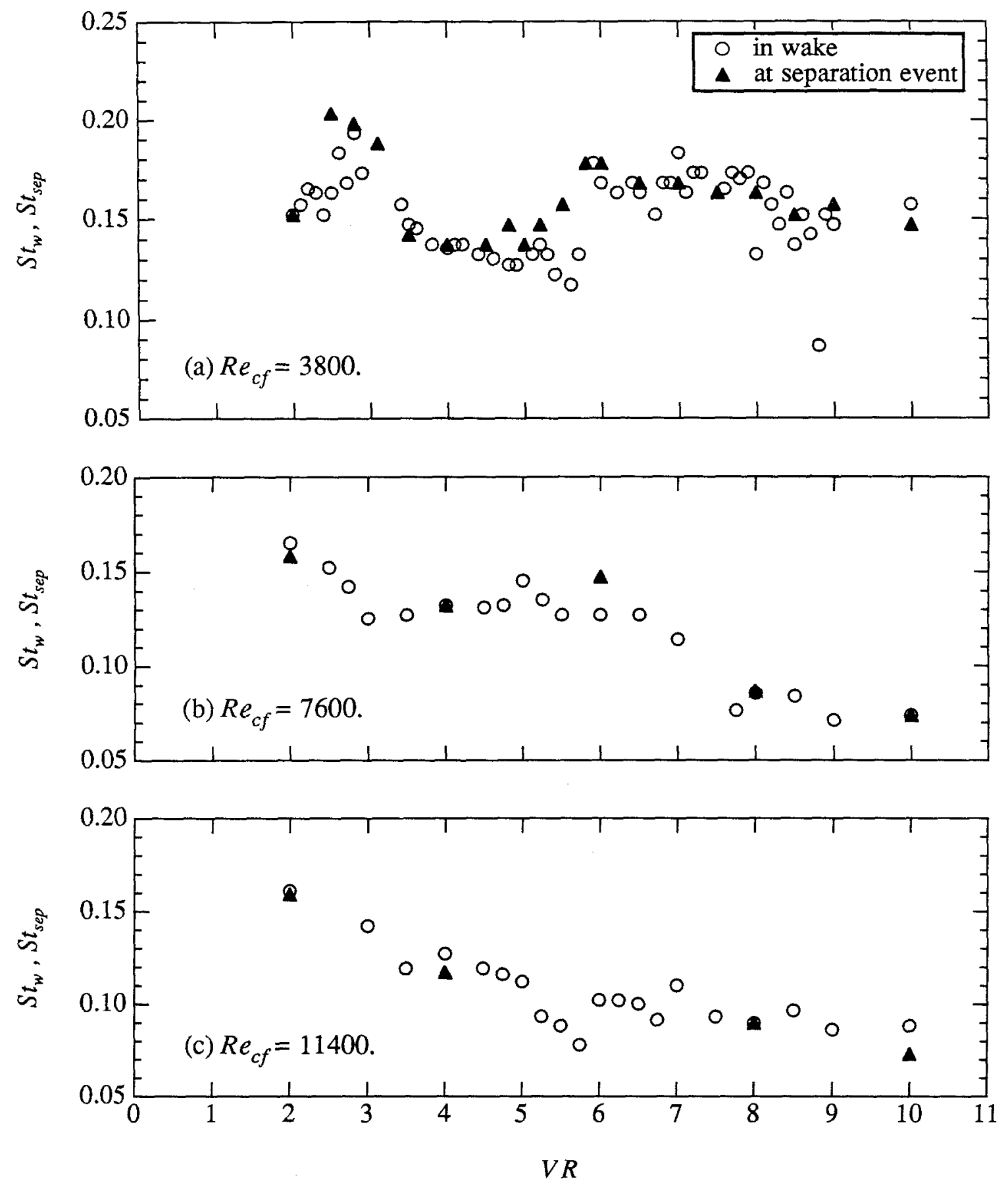

FIGURE 5.11: Comparison of $S t_{w}$ with $S t_{\text {sep }}$. (a) $R e_{c f}=3800$, (b) $R e_{c f}$ $=7600$, (c) $R e_{c f}=11400$. St $t_{w}$ measured at $X / D_{j}=3.5, Y / D_{j}$ $=1.5, Z / D_{j}=.5$. $S t_{\text {sep }}$ measured near the crossflow boundary layer separation events. 

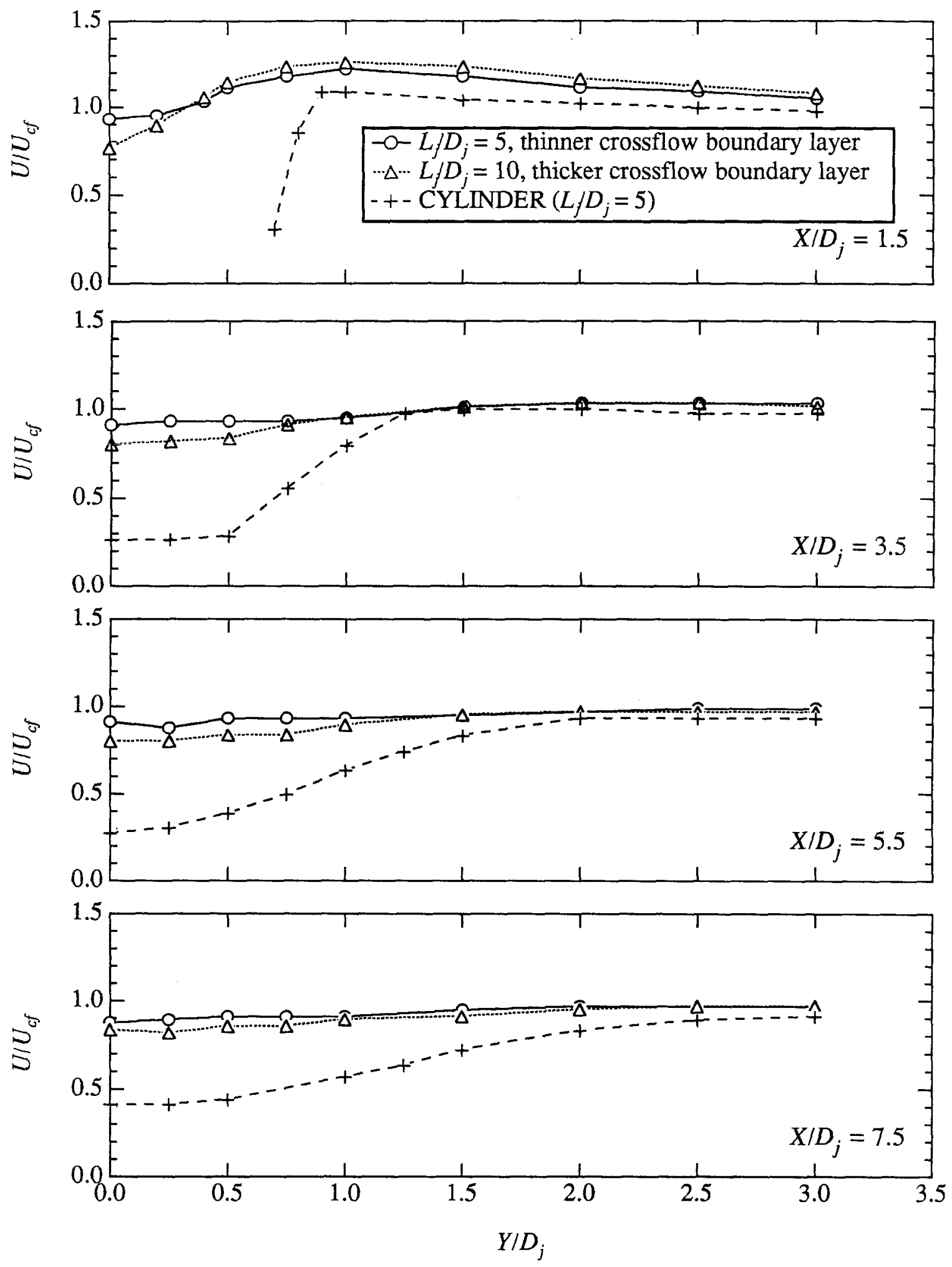

FIGURE 5.12: Wake velocity profiles at $Z / D_{j}=.5 . V R=4 . R e_{c f}=3800$. 

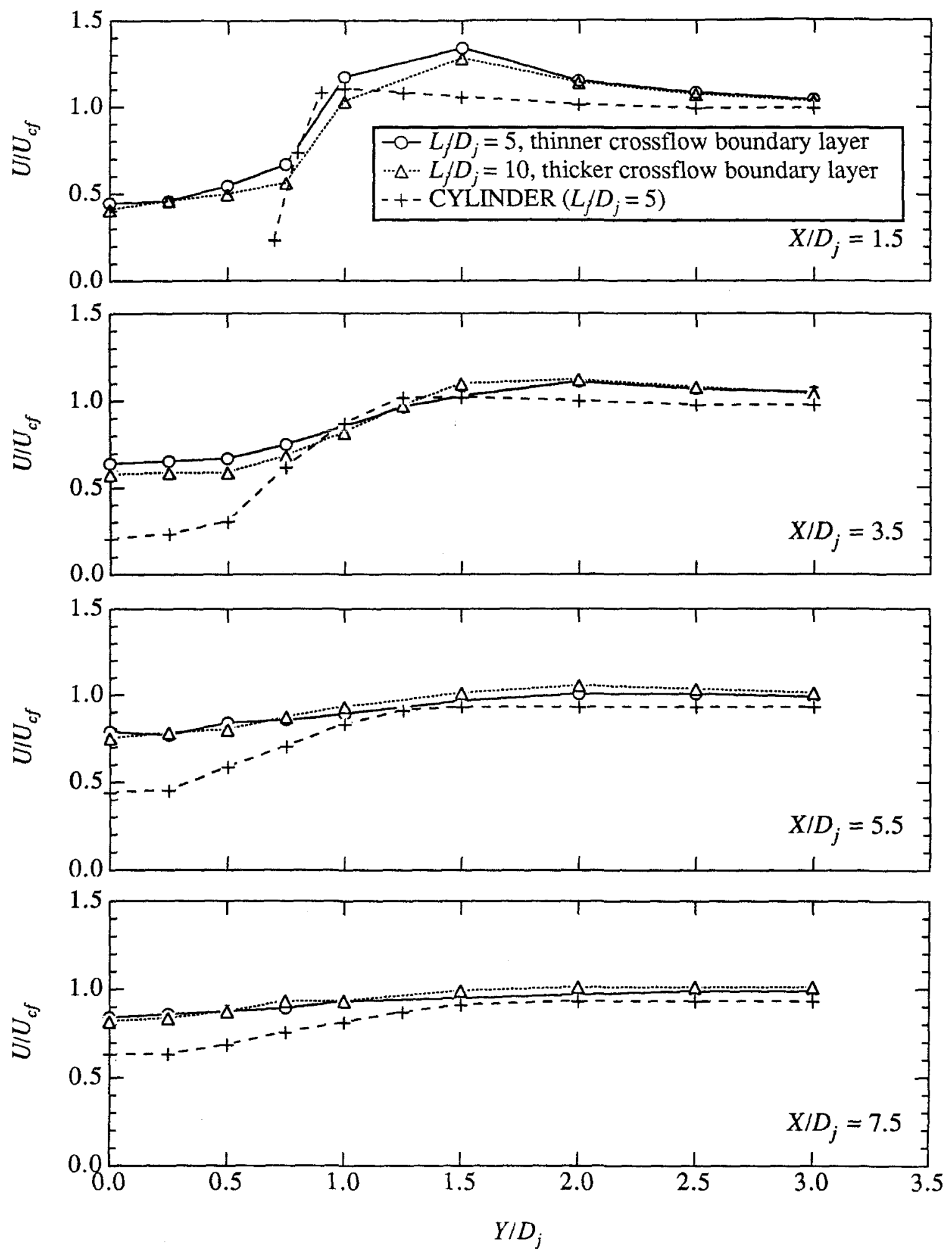

FIGURE 5.13: Wake velocity profiles at $Z / D_{j}=2.5 . \quad V R=4 . R e_{c f}=3800$. 

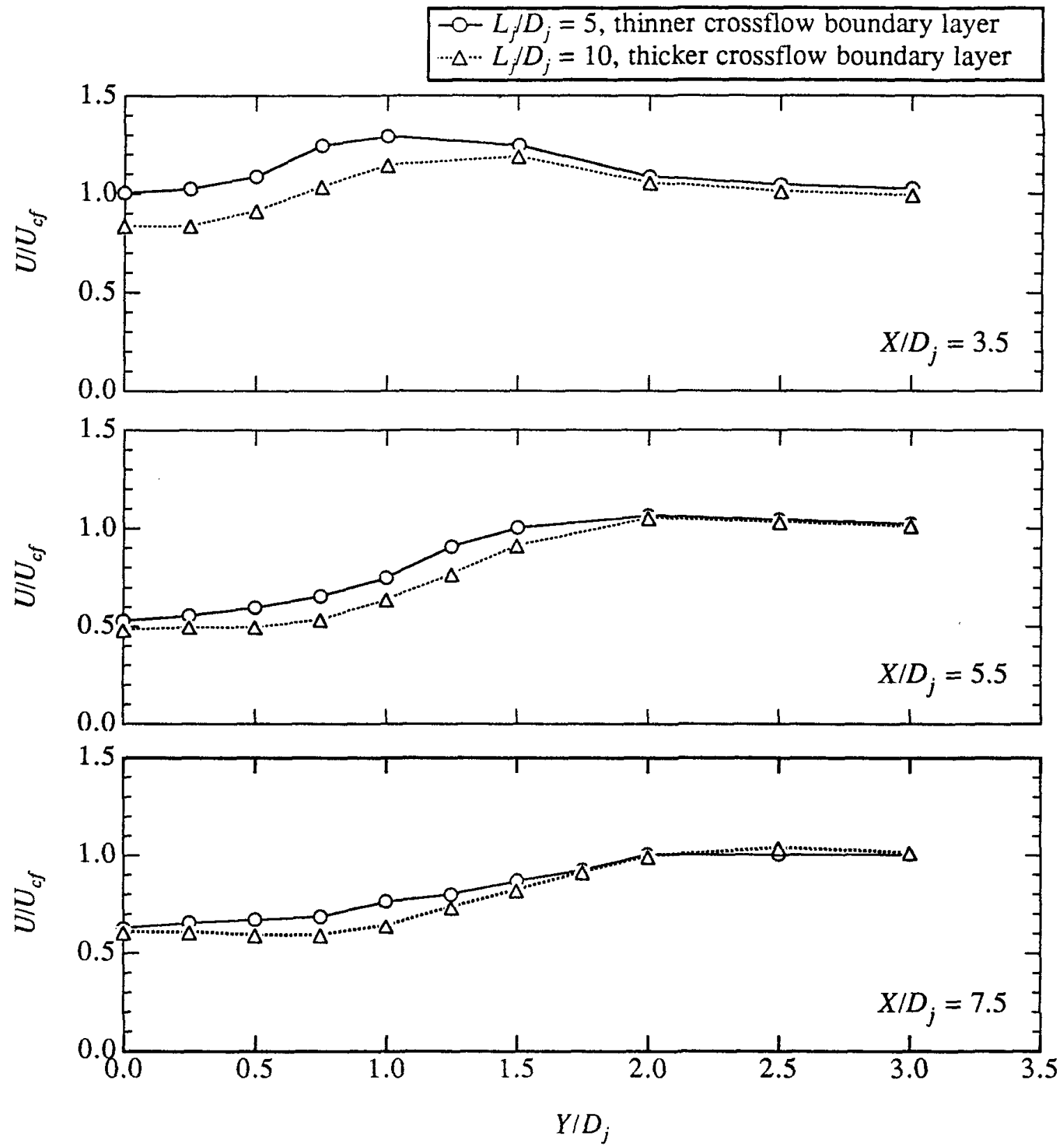

FIGURE 5.14: Wake velocity profiles at $Z / D_{j}=4.5 . V R=4 . R e_{c f}=3800$. 

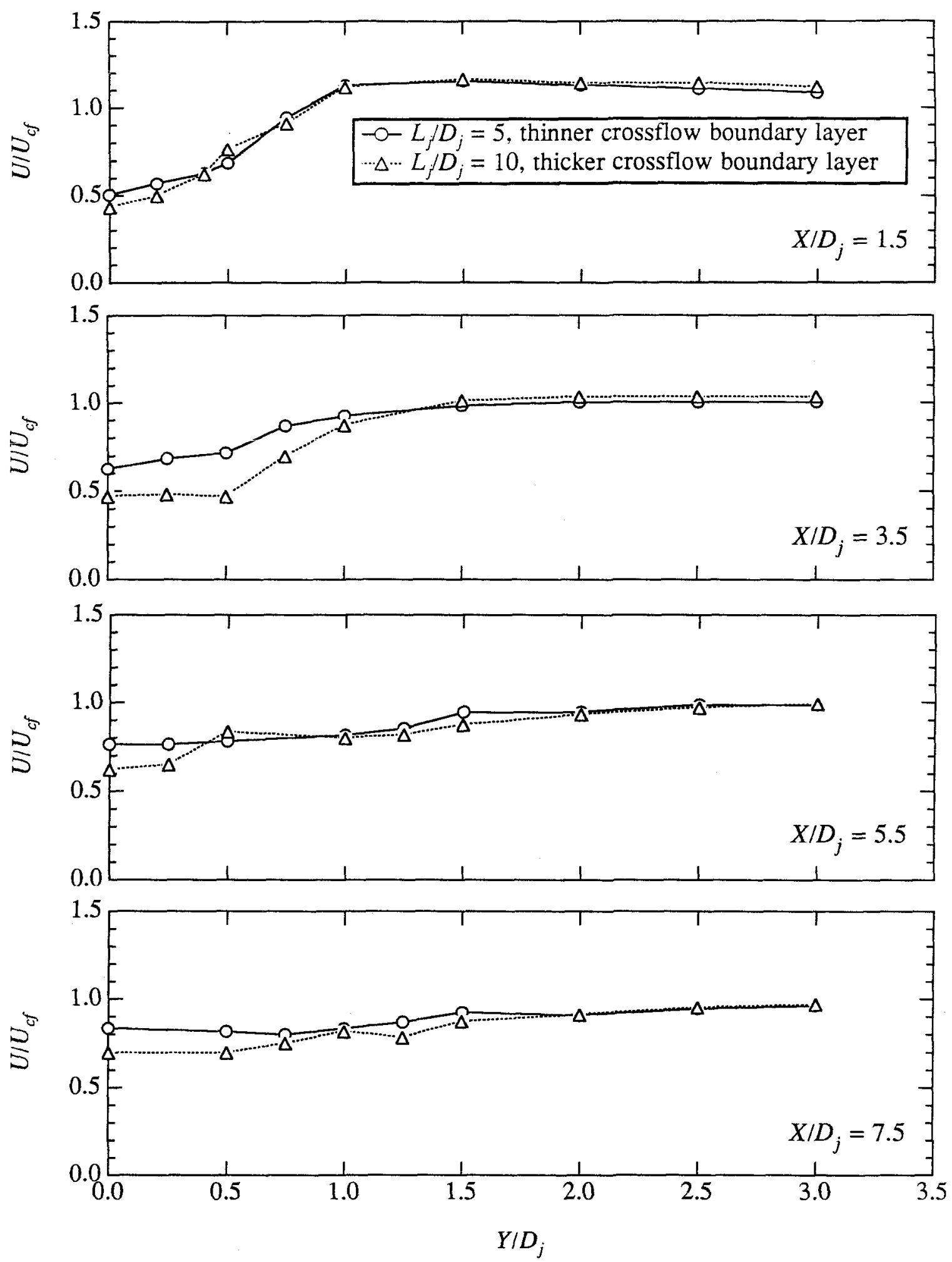

FIGURE 5.15: Wake velocity profiles at $Z / D_{j}=.5 . V R=8 . R e_{c f}=3800$. 

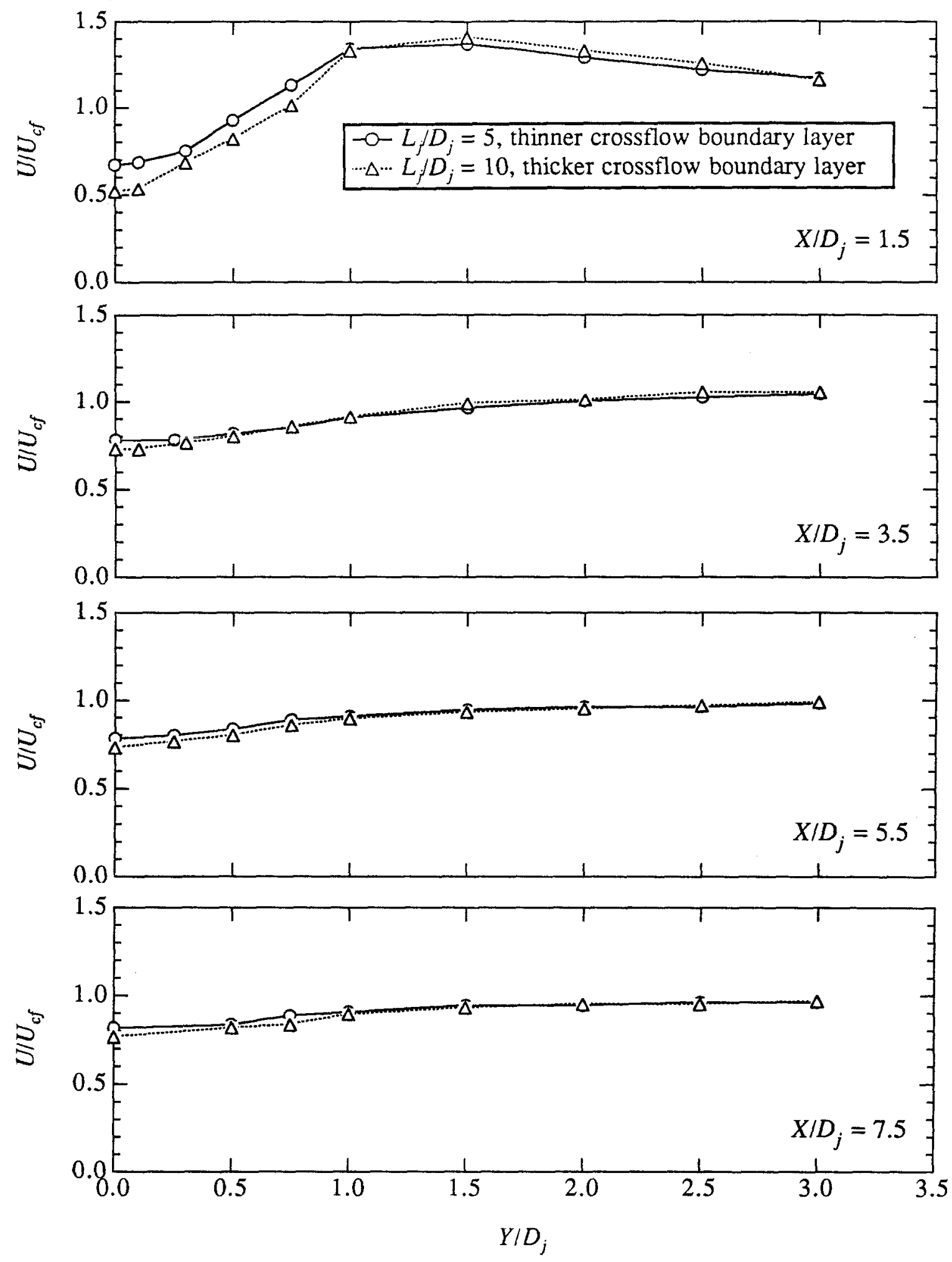

FIGURE 5.16: Wake velocity profiles at $Z / D_{j}=2.5 . V R=8 . R e_{c f}=3800$ 

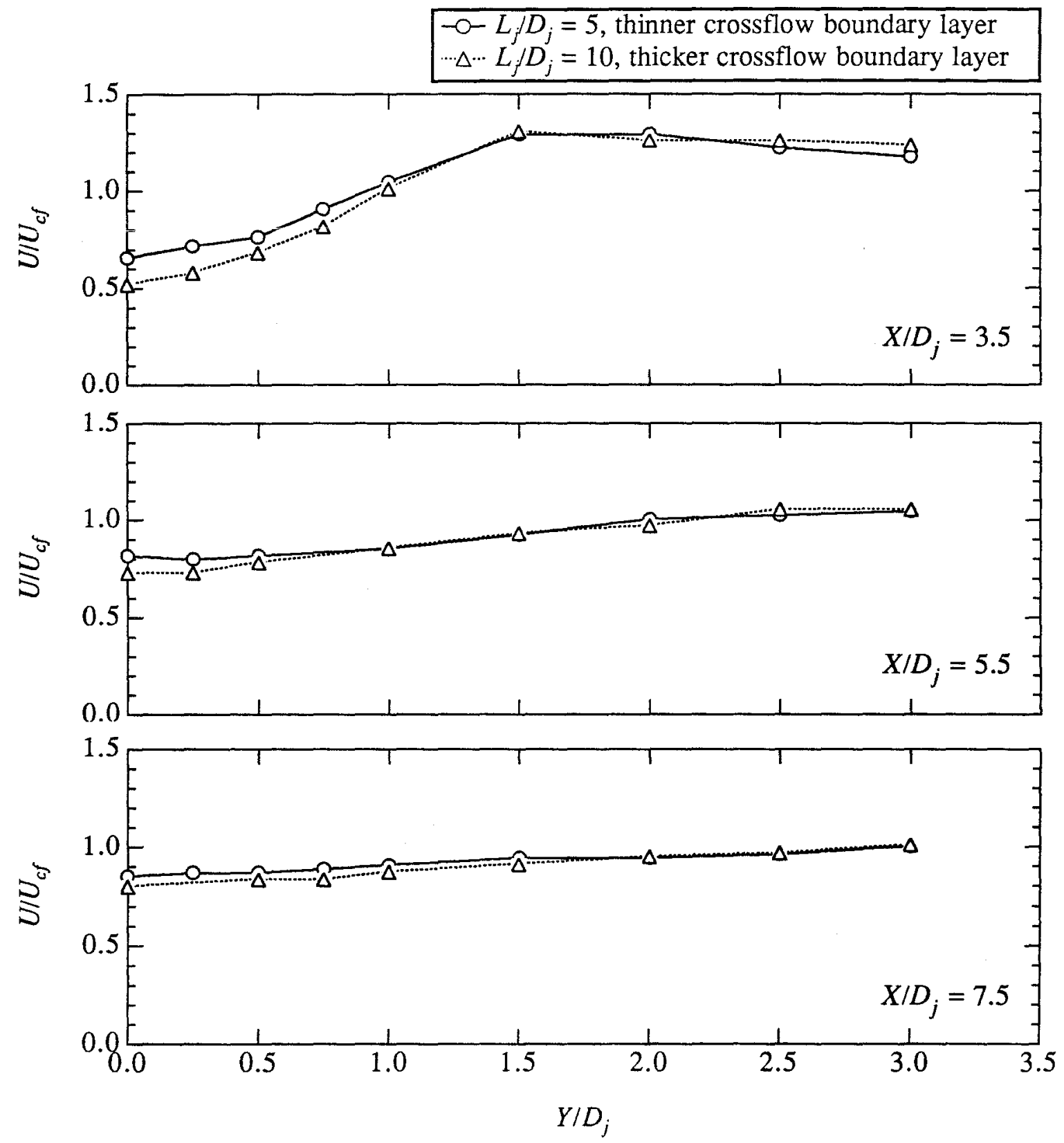

FIGURE 5.17: Wake velocity profiles at $Z / D_{j}=4.5 . \quad V R=8 . R e_{c f}=3800$. 


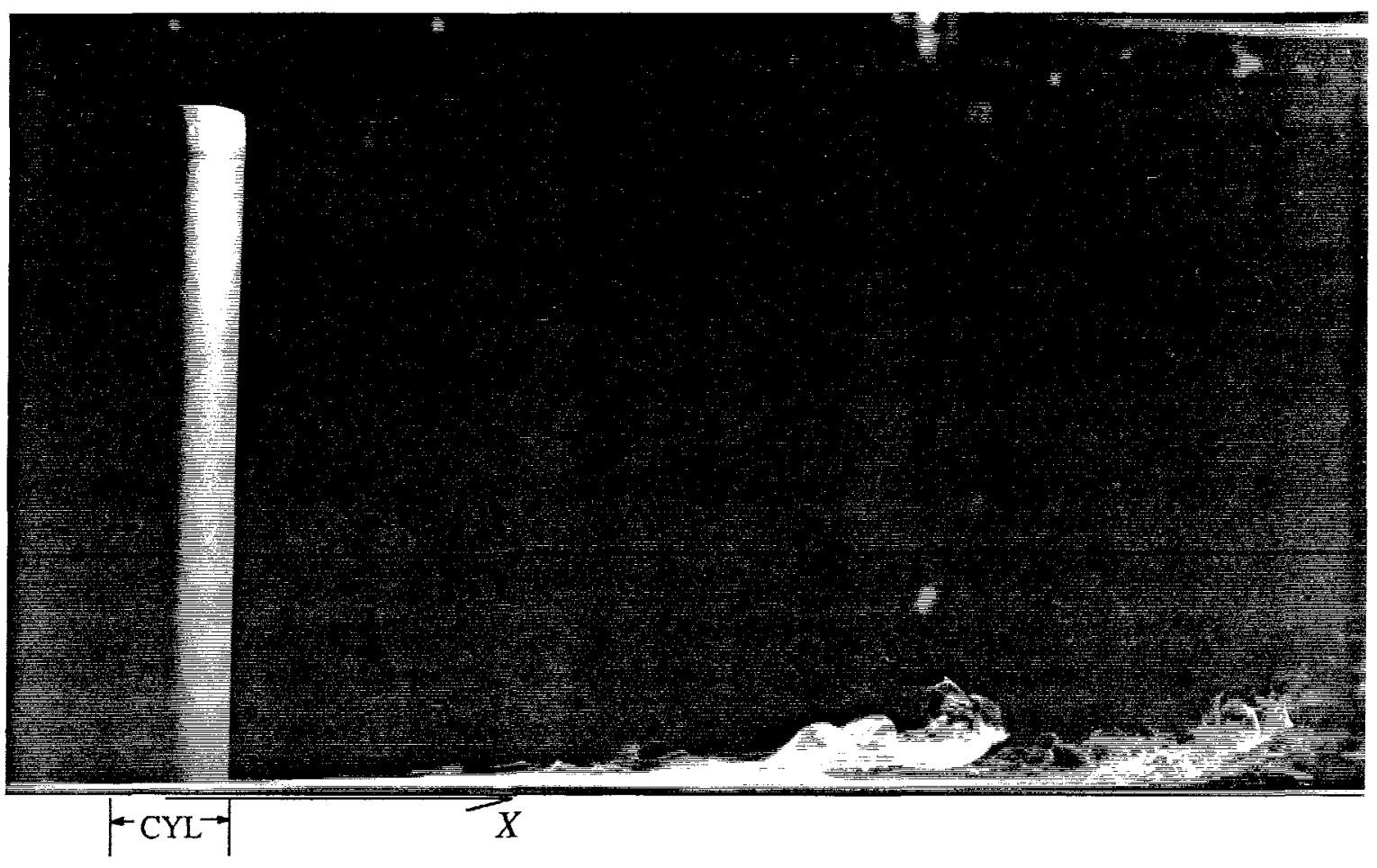

FIGURE 5.18: Side view of flow about a circular cylinder. $A R=6 . R e_{c f}=3800$. $Z_{s w} / D_{j}=0+$. 


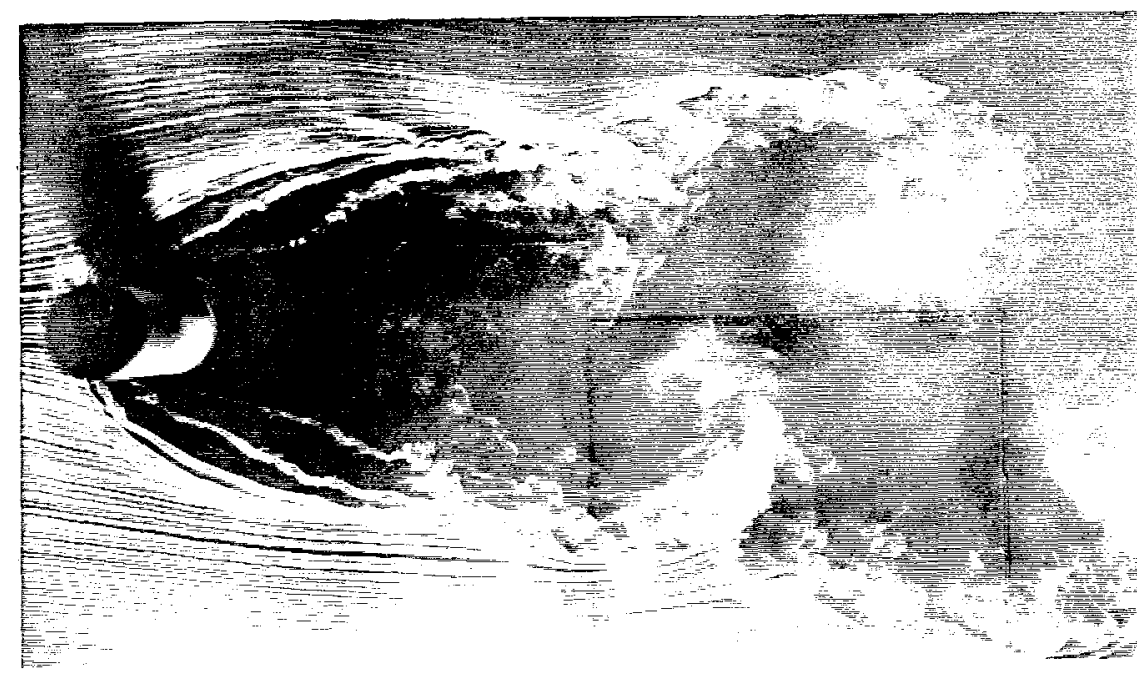

(a) $A R=6$

cylinder.

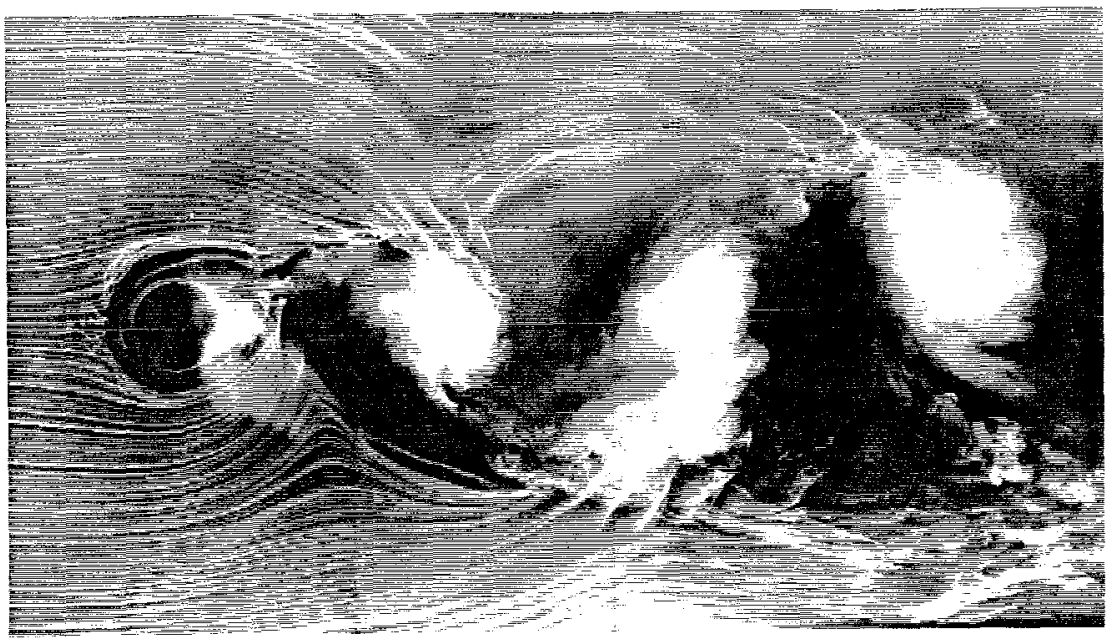

(b) $V R=4$

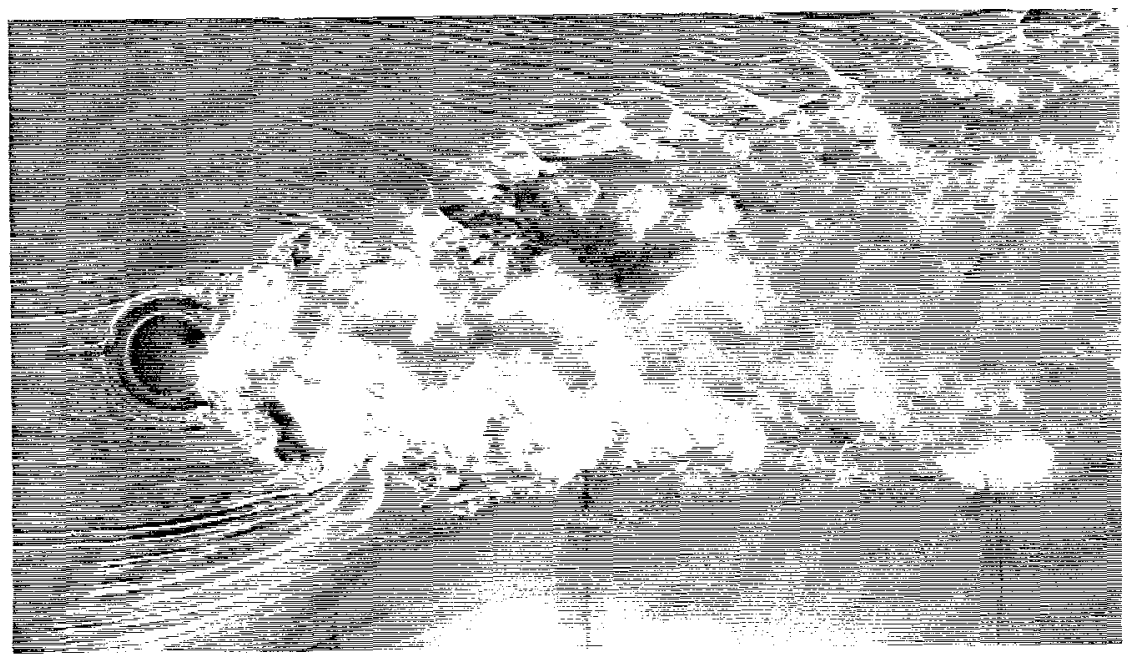

(c) $V R=8$.

FIGURE 5.19: Cross sectional views of wakes with $Z_{s w} / D_{j}=Z_{s w} / D_{c}=0+$. $R e_{c f}=11400$. 


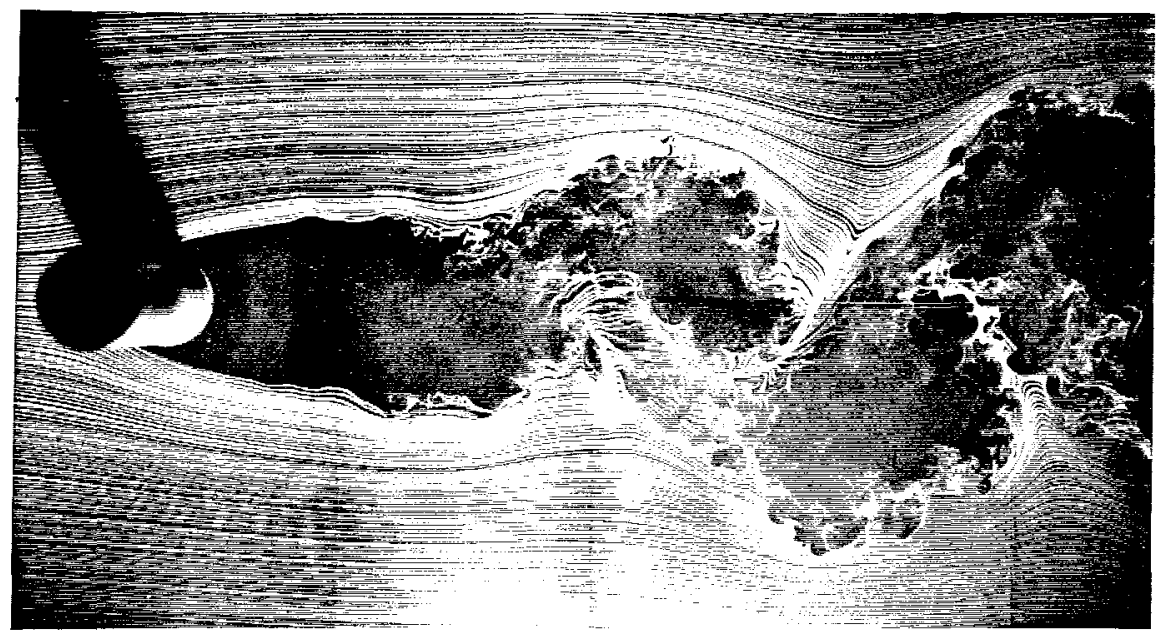

(a) $A R=6$

cylinder.

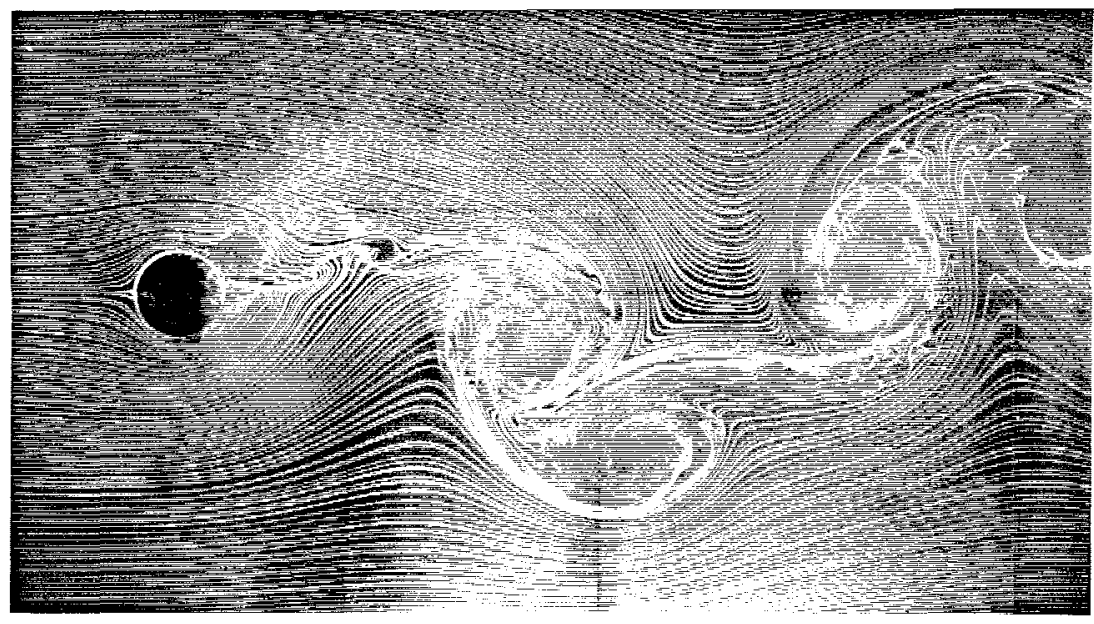

(b) $V R=4$.

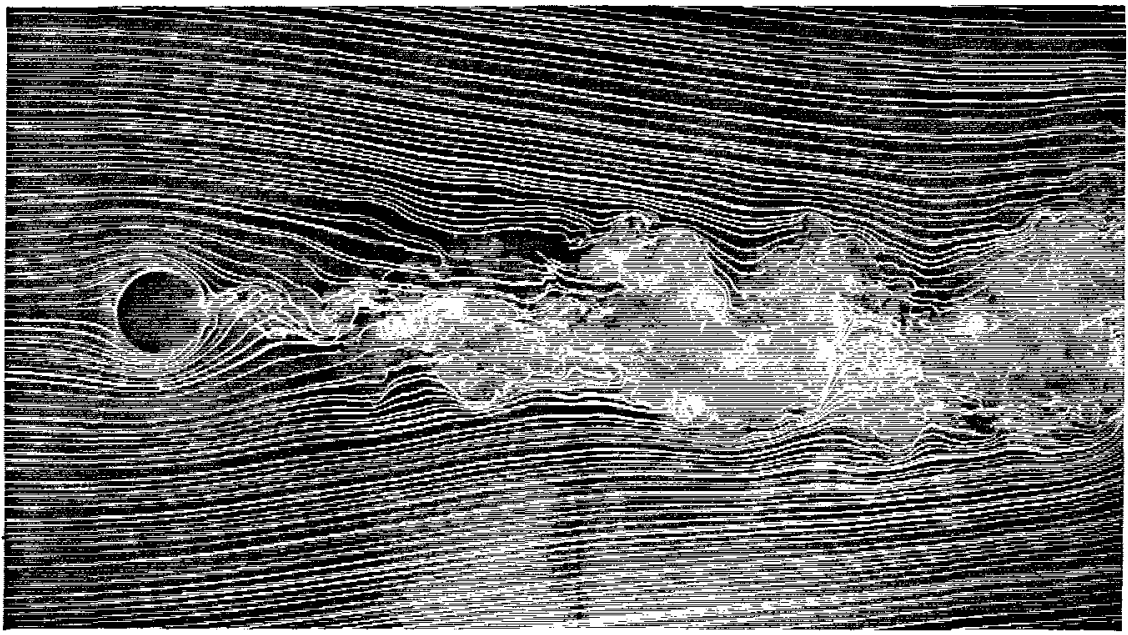

(c) $V R=8$.

FIGURE 5.20: Cross sectional views of wakes with $Z_{s w} / D_{j}=Z_{s w} / D_{c}=.5$. $R e_{c f}=11400$. 


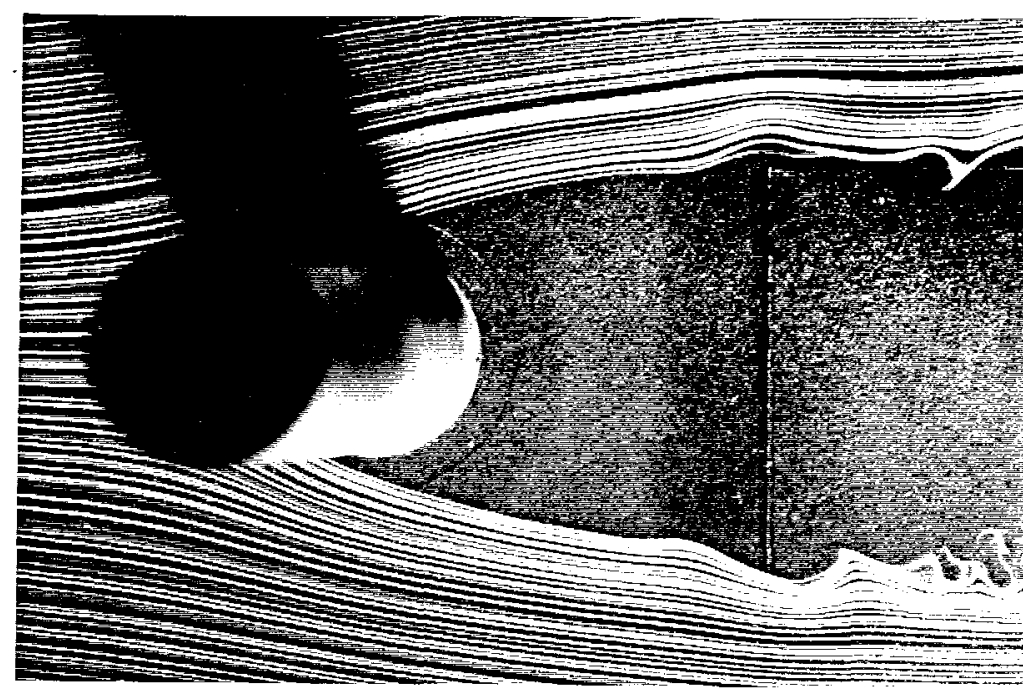

(a) $A R=6$

cylinder.

$Z_{s w} / D_{c}=.5$.

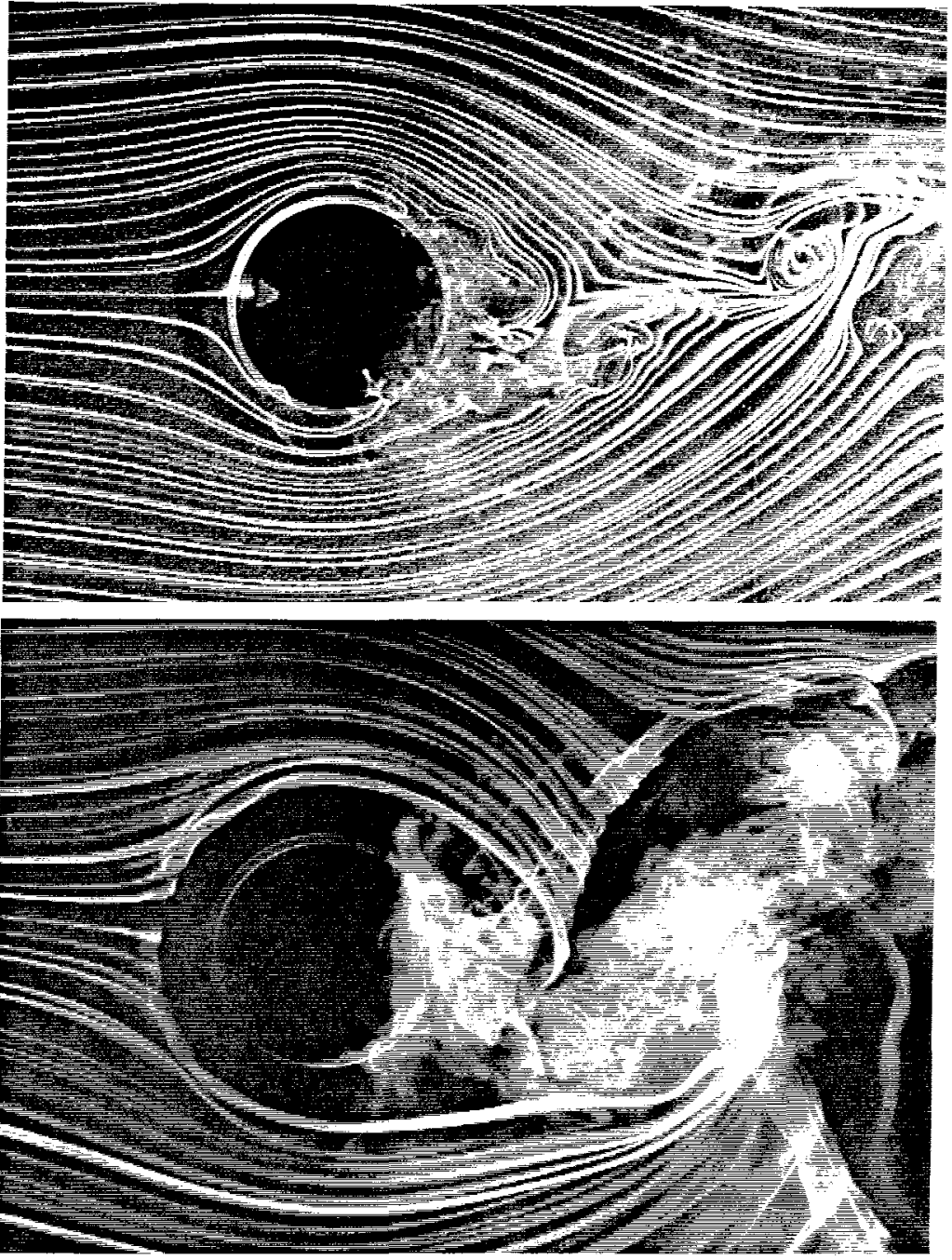

(b) $V R=4$.

$Z_{s w} / D_{j}=.5$.

(c) $V R=4$.

$Z_{s w} / D_{j}=0+$.

FIGURE 5.21: Comparison of flow around a cylinder with the flow around a jet. $R e_{c f}=3800$. 


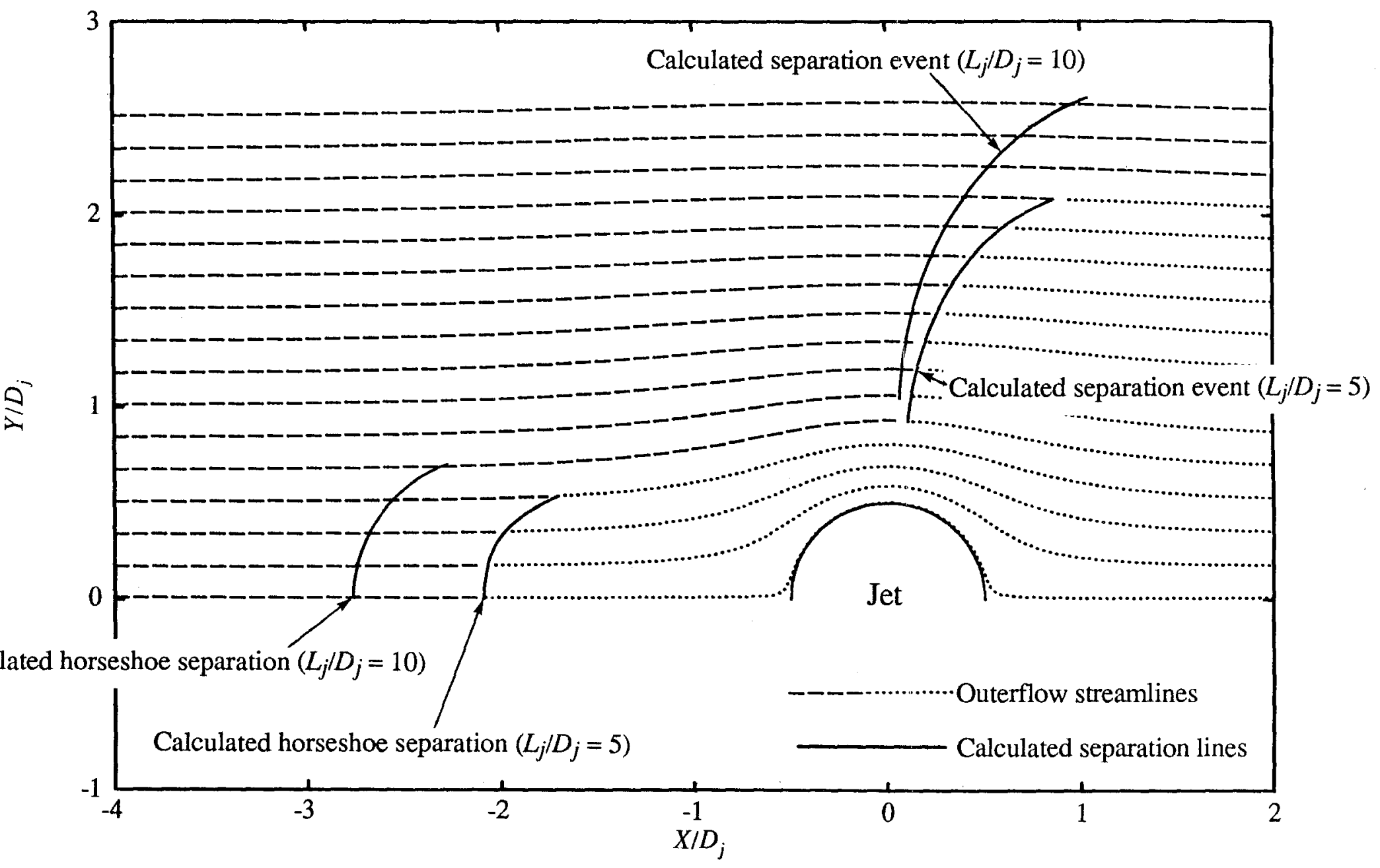

FIGURE 5.22: Predicted separations using a Thwaites method calculation of the crossflow boundary layer with a potential flow pressure gradient imposed on it. 


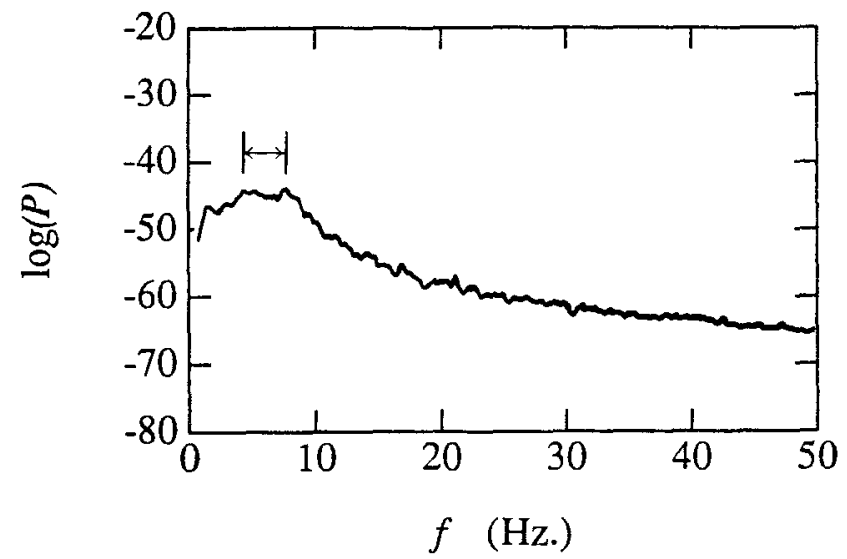

(a) sharpness $=0$.
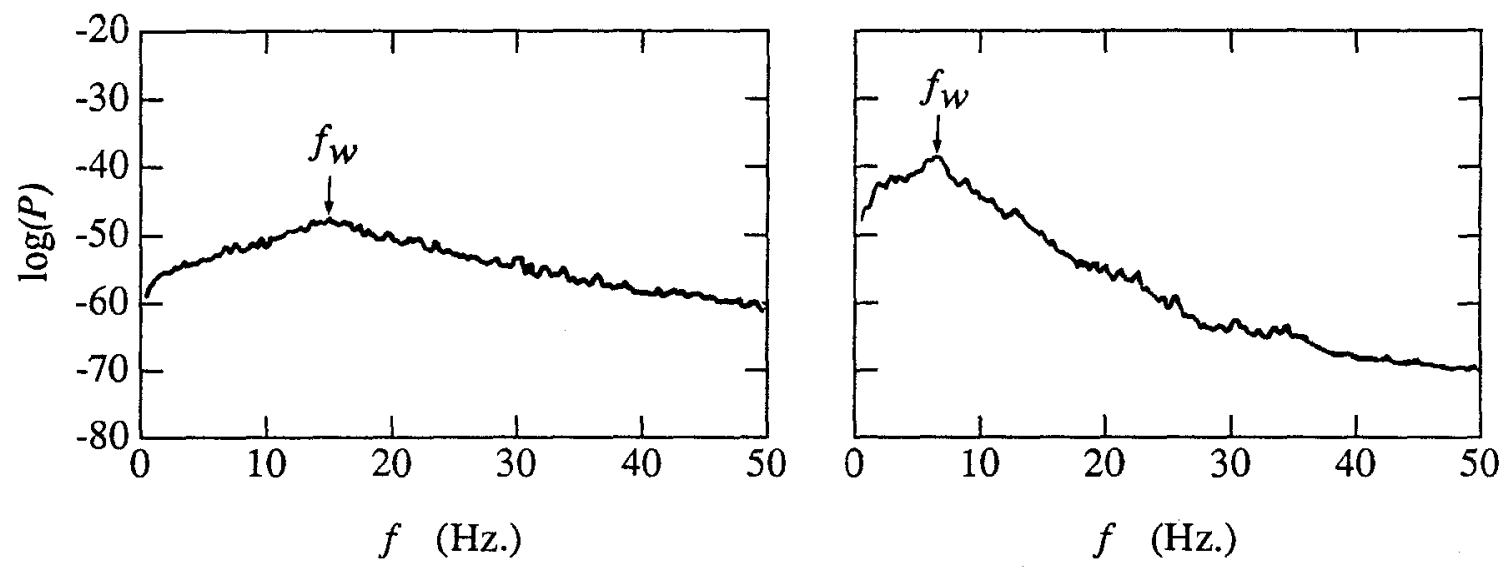

(b) sharpness $=1$.

(c) sharpness $=2$.
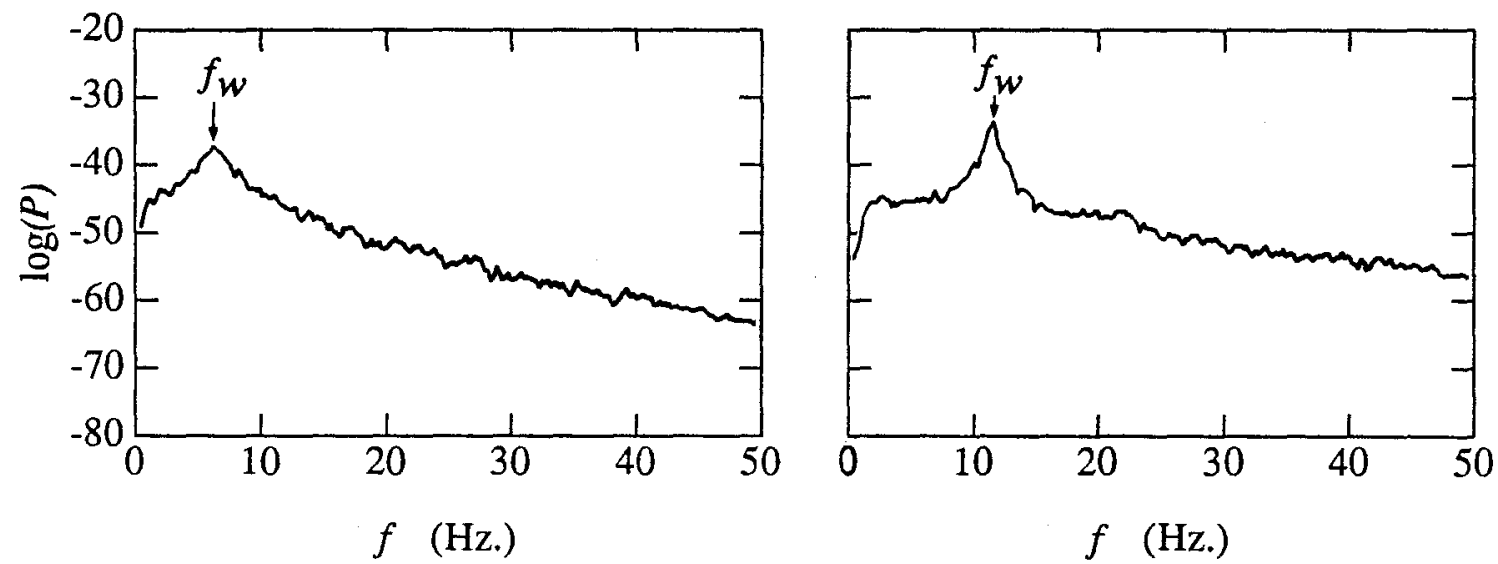

(d) sharpness $=3$.

(e) sharpness $=4$.

FIGURE 5.23: Definitions of assigned spectral sharpness levels. (a) sharpness $=0$,

(b) sharpness $=1$, (c) sharpness $=2$, (d) sharpness $=3$,

(e) sharpness $=4$. 


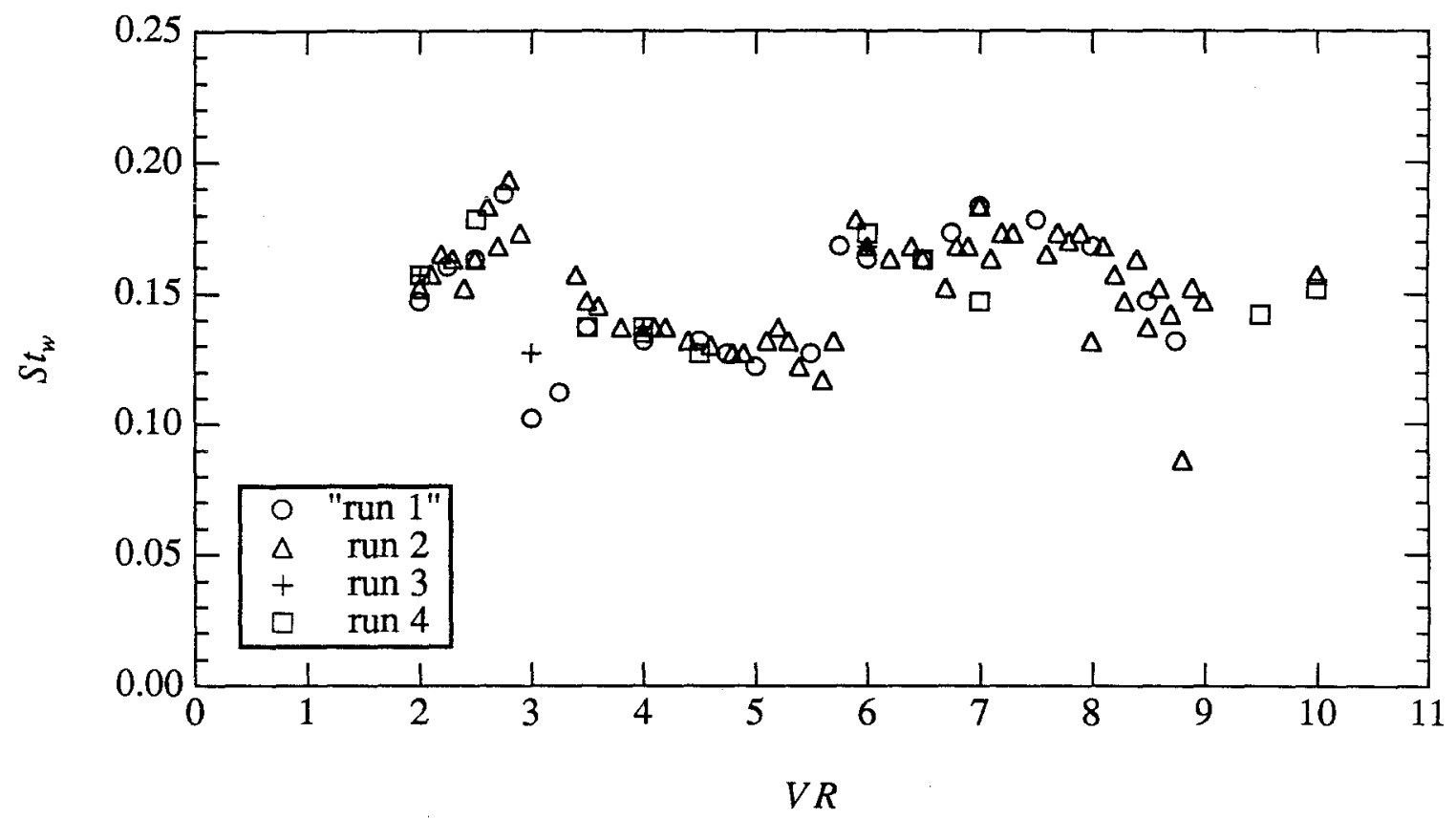

FIGURE 5.24: The degree of repeatibility of the wake Strouhal number data. $R e_{c f}=3800 . L_{j} / D_{j}=5$. 

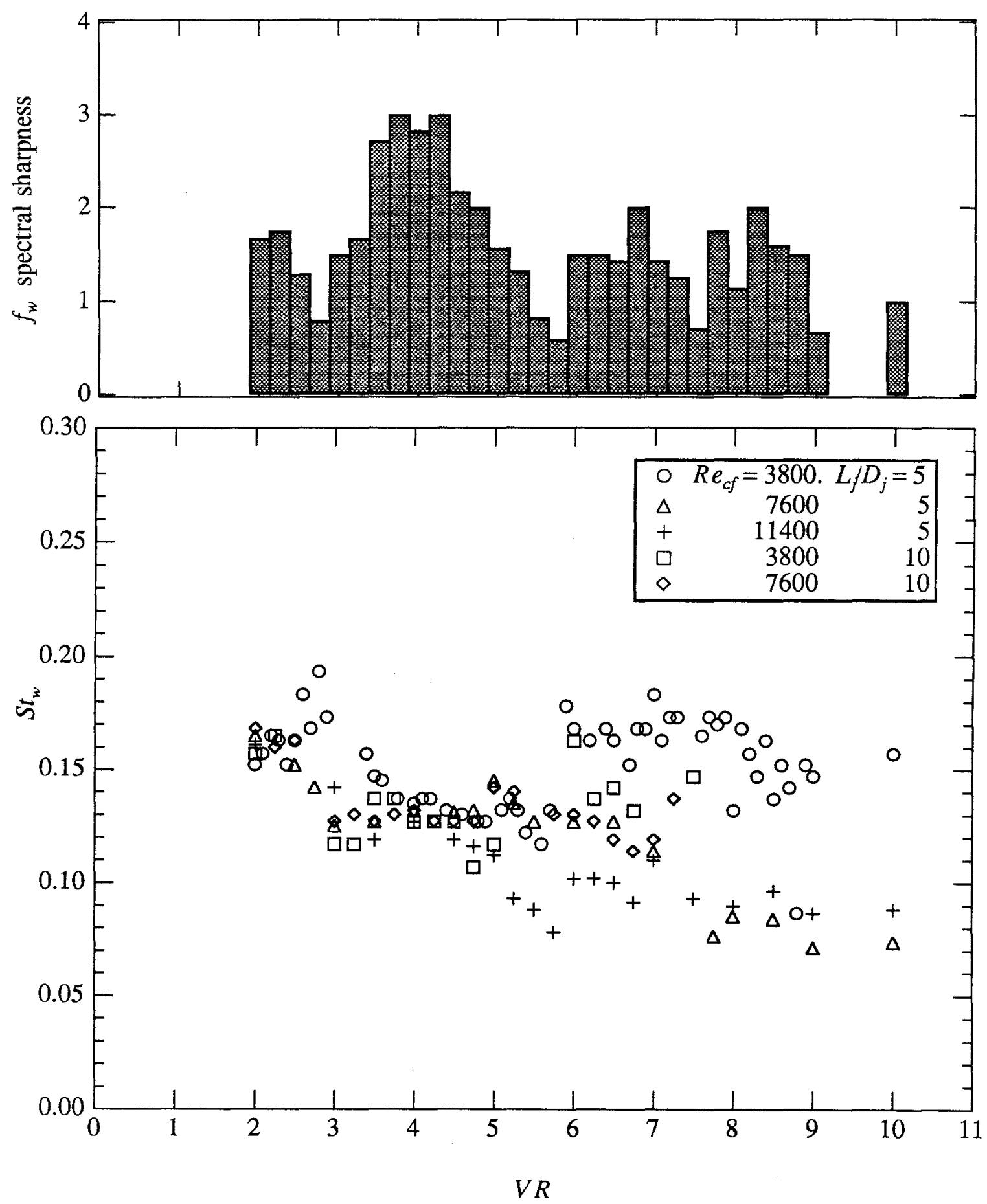

FIGURE 5.25: Compilation of wake Strouhal numbers for several combinations of $R e_{c f}$ and $L_{j} / D_{j}$. Average spectral sharpnesses are also shown. 


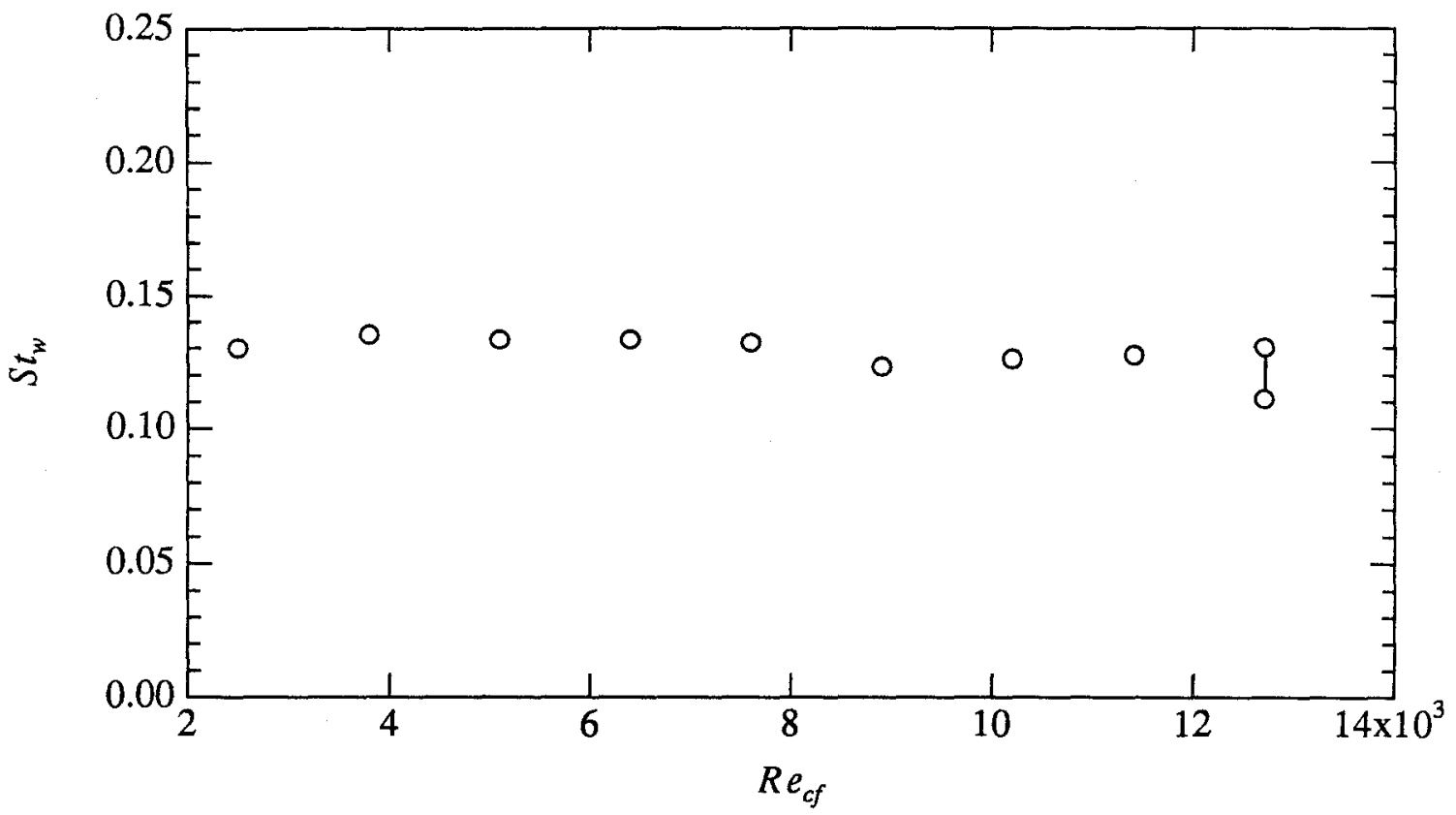

FIGURE 5.26: Strouhal number as a function of $R e_{c f}$ at $V R=4 . L_{j} / D_{j}=5$. 
185
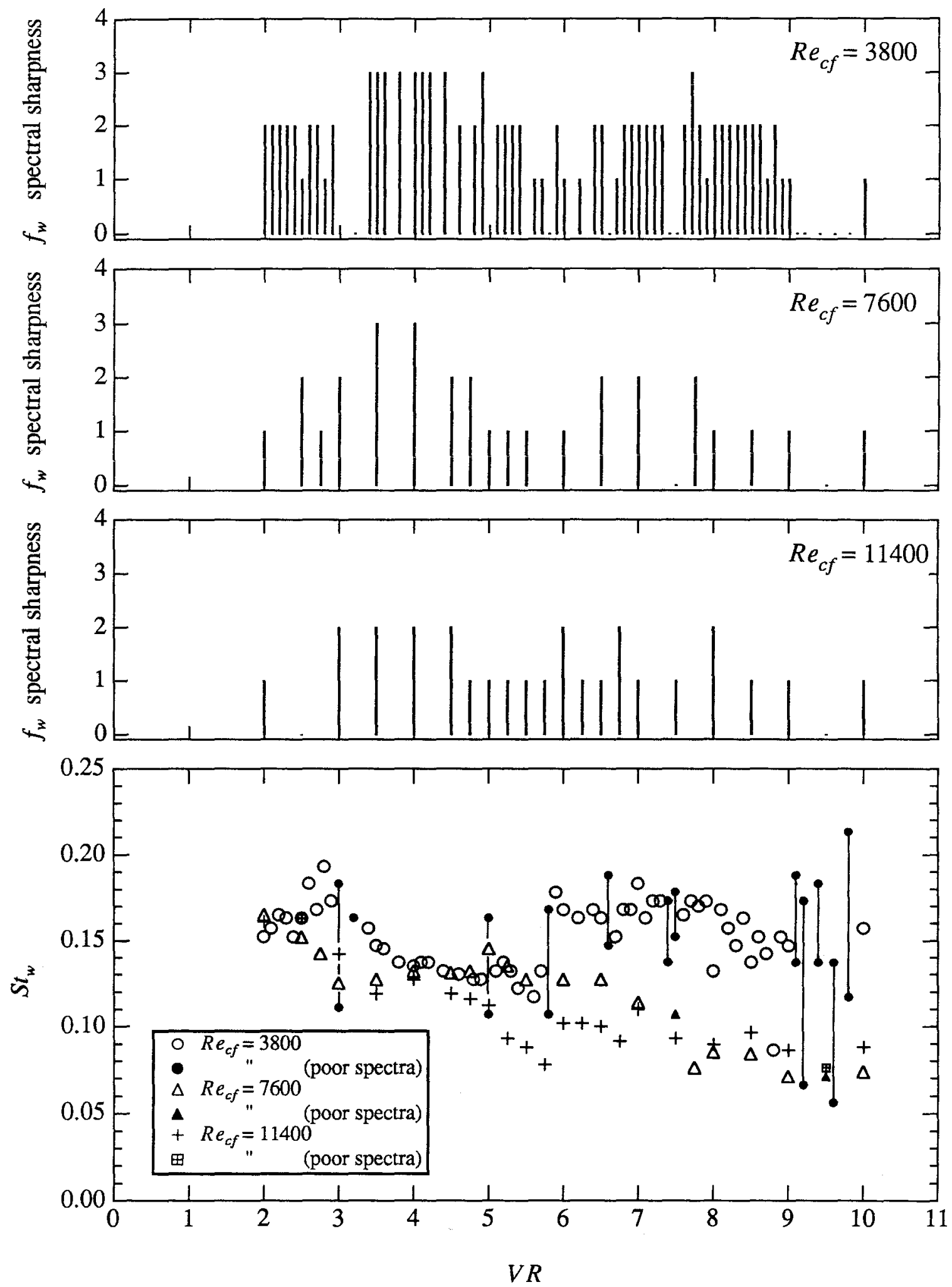

FIGURE 5.27: Wake Strouhal numbers at 3 crossflow Reynolds numbers. $L_{j} / D_{j}=5$. 

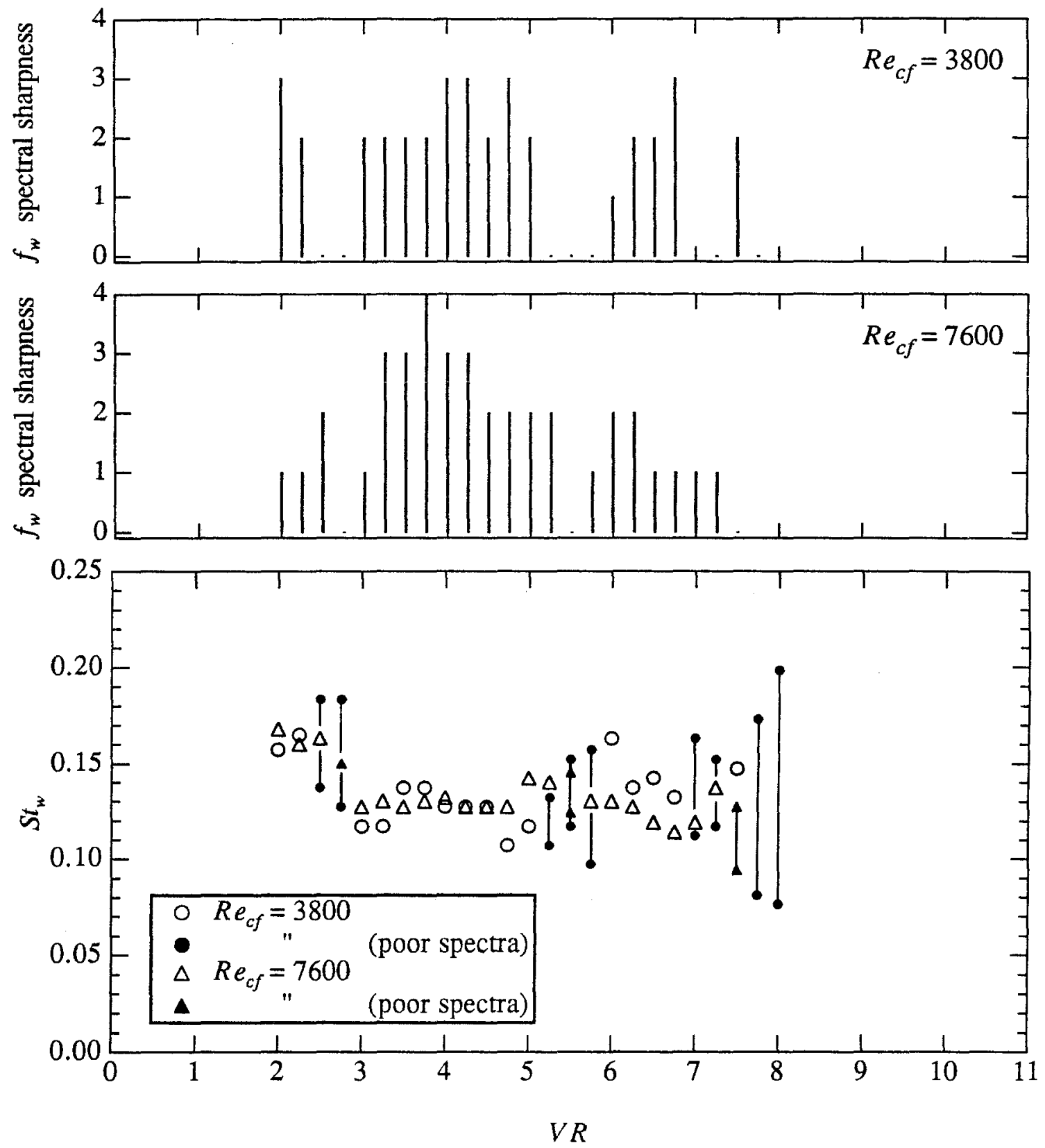

FIGURE 5.28: Wake Strouhal numbers at 3 crossflow Reynolds numbers. $L_{j} / D_{j}=10$. 


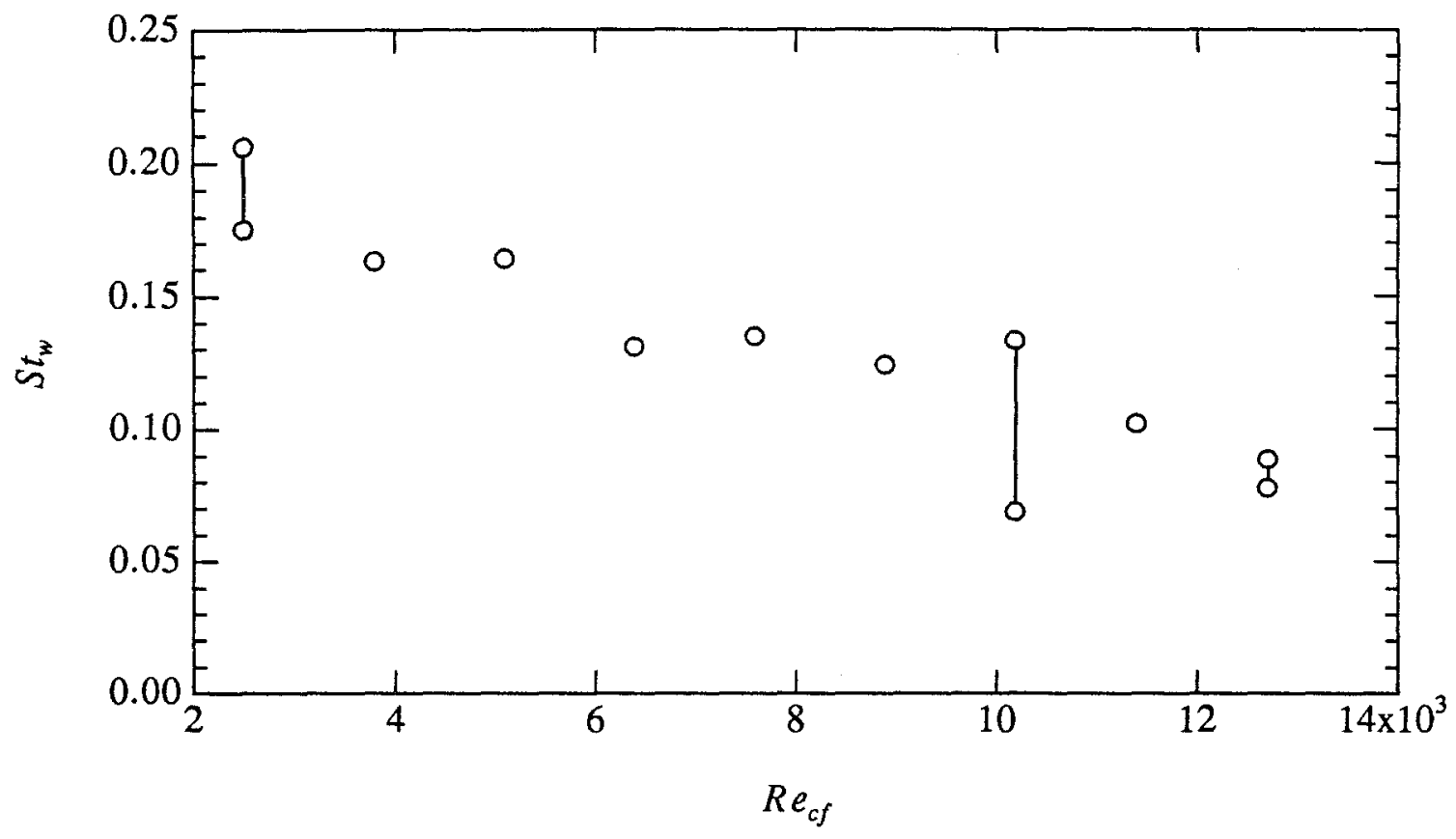

FIGURE 5.29: Strouhal number as a function of $R e_{c f}$ at $V R=6.25 . \quad L_{j} / D_{j}=5$. 

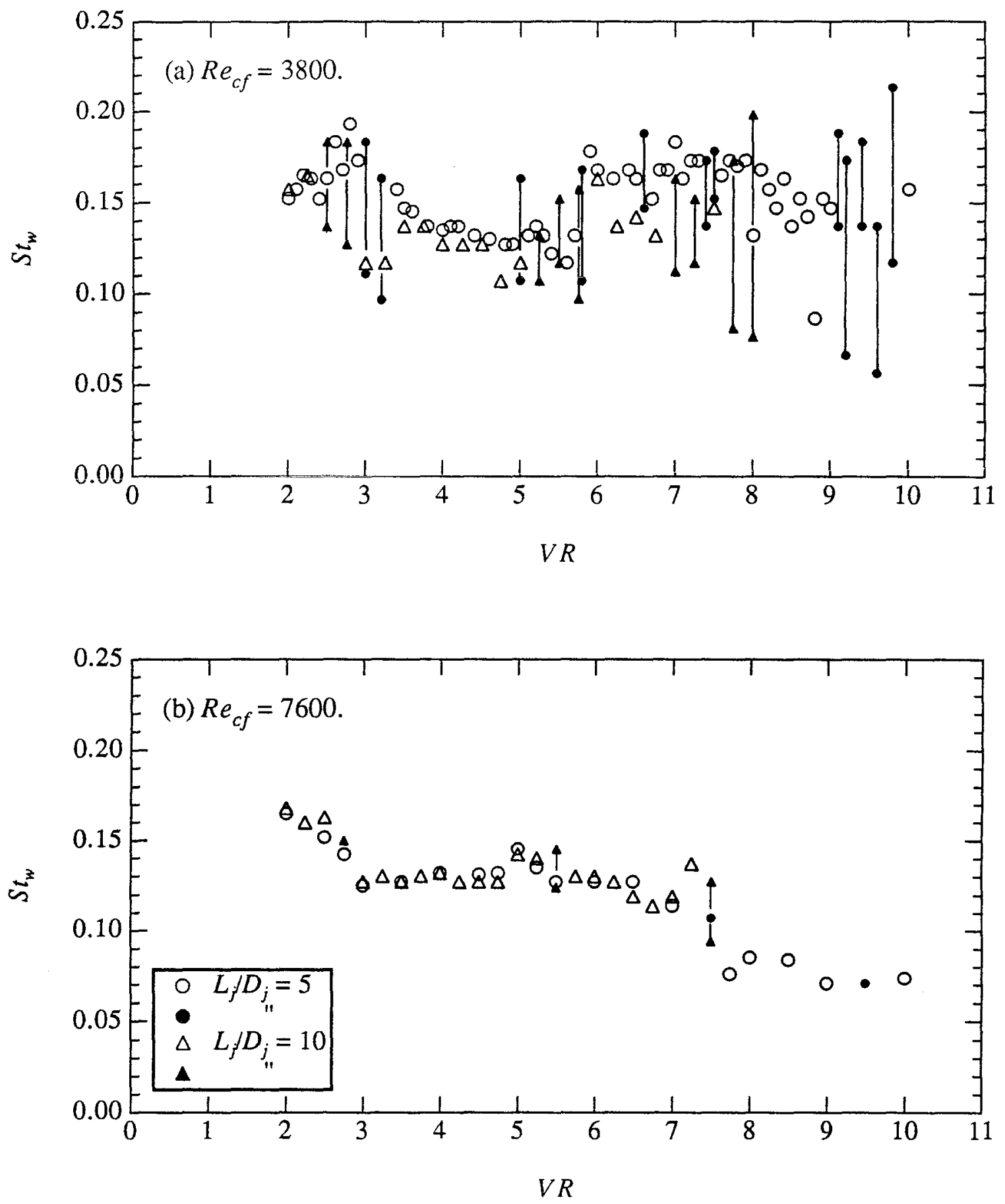

FIGURE 5.30: Wake Strouhal numbers at $L_{j} / D_{j}=5 \& 10$.

(a) $R e_{c f}=3800$, (b) $R e_{c f}=7600$. 


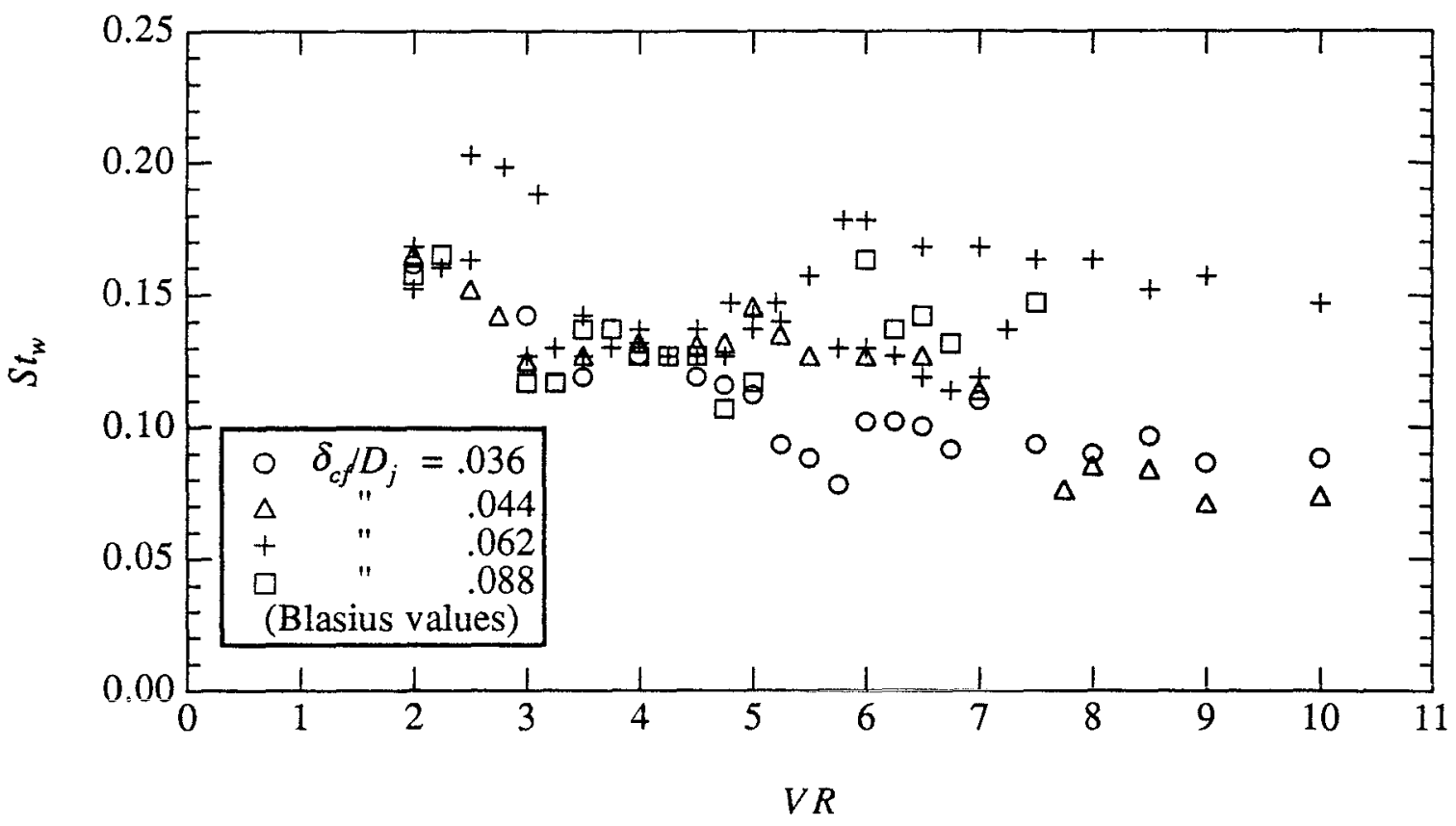

FIGURE 5.31: Wake Strouhal numbers at several $\delta_{c f} D_{j}$.

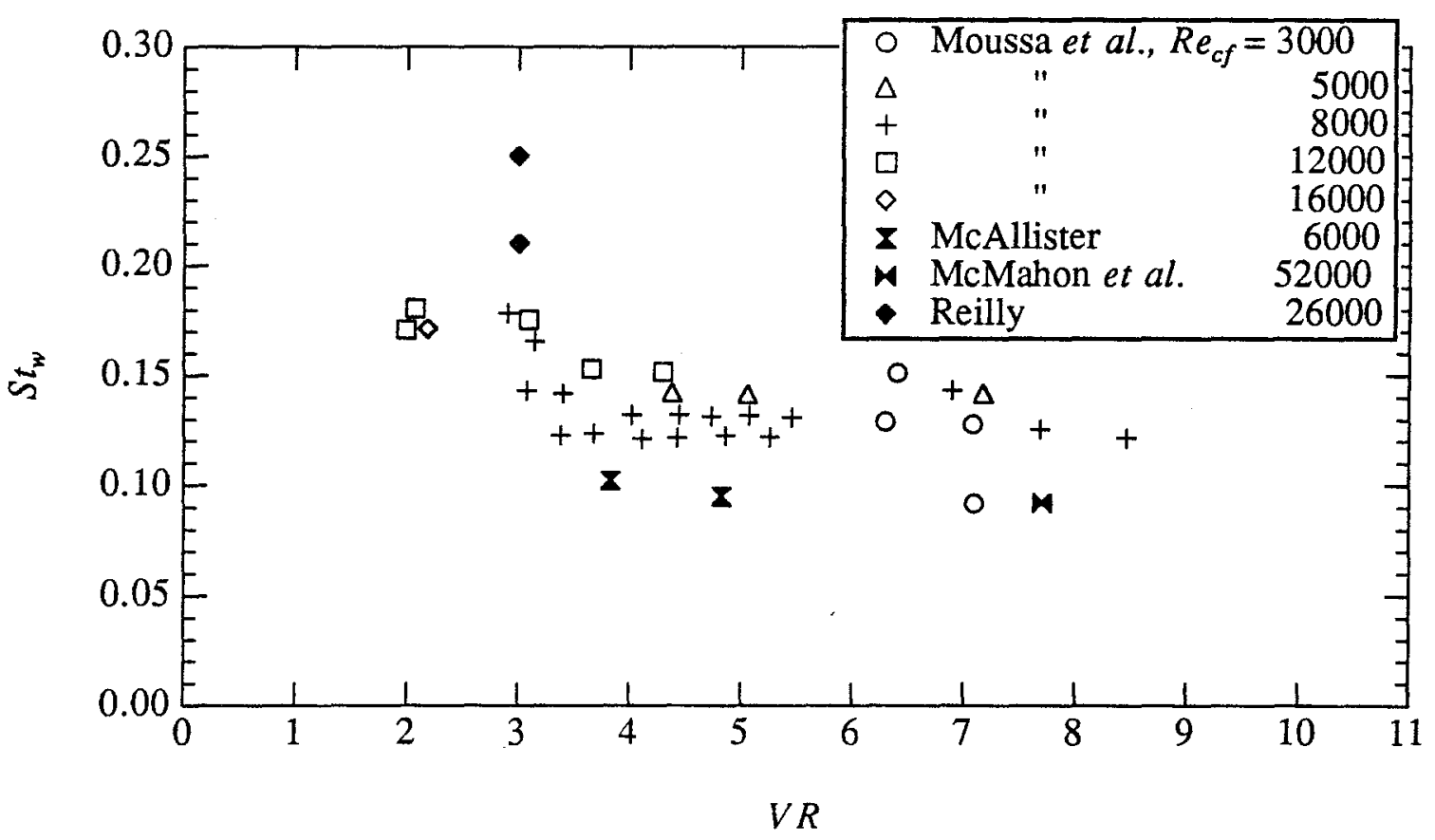

FIGURE 5.32: Wake Strouhal numbers from several references. 
190

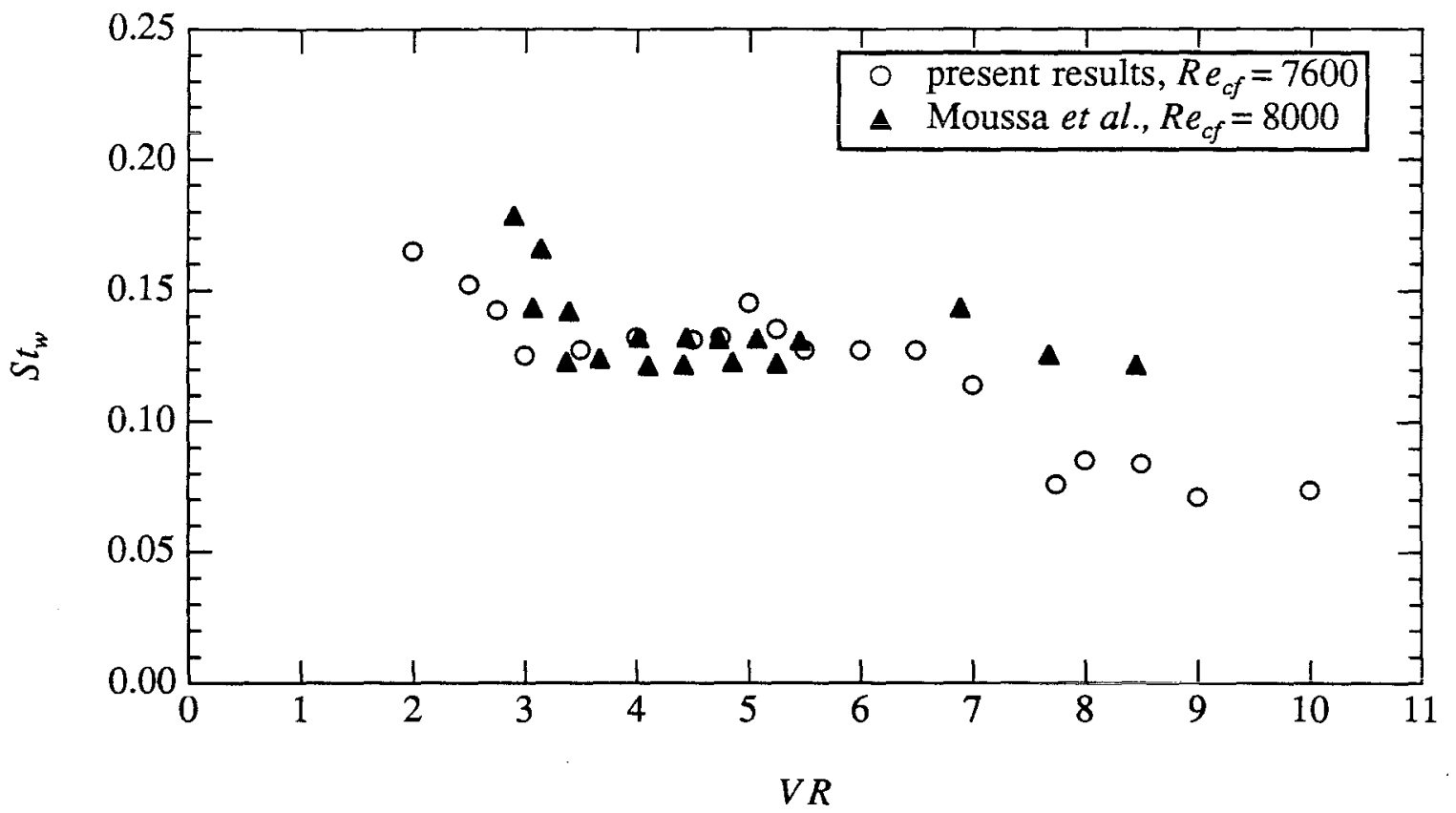

FIGURE 5.33: Comparison of present wake Strouhal numbers with those of Moussa et al. (1977).

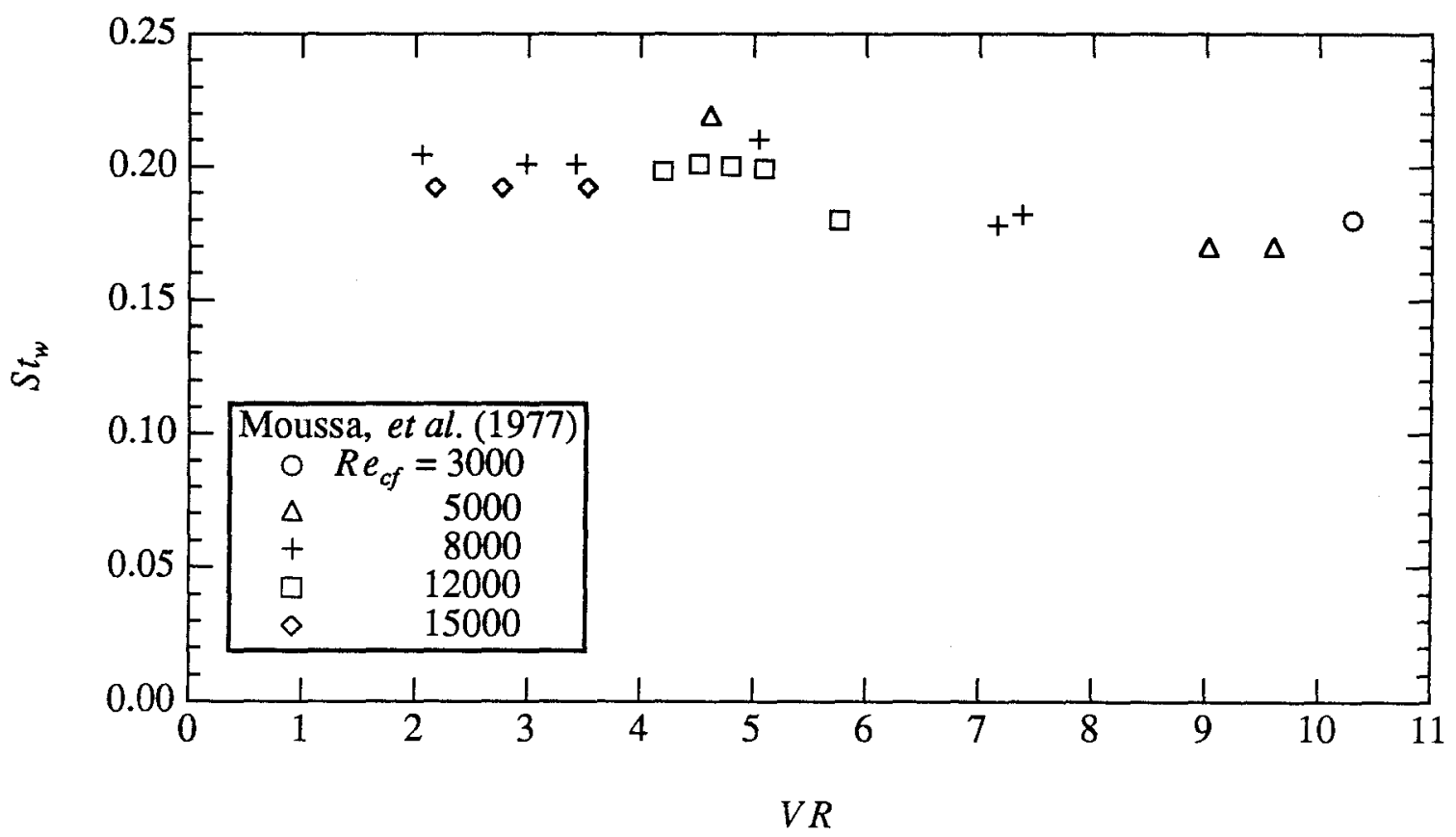

FIGURE 5.34: Wake Strouhal numbers of Moussa et al. for a jet whose supply pipe protrudes into the crossflow. 


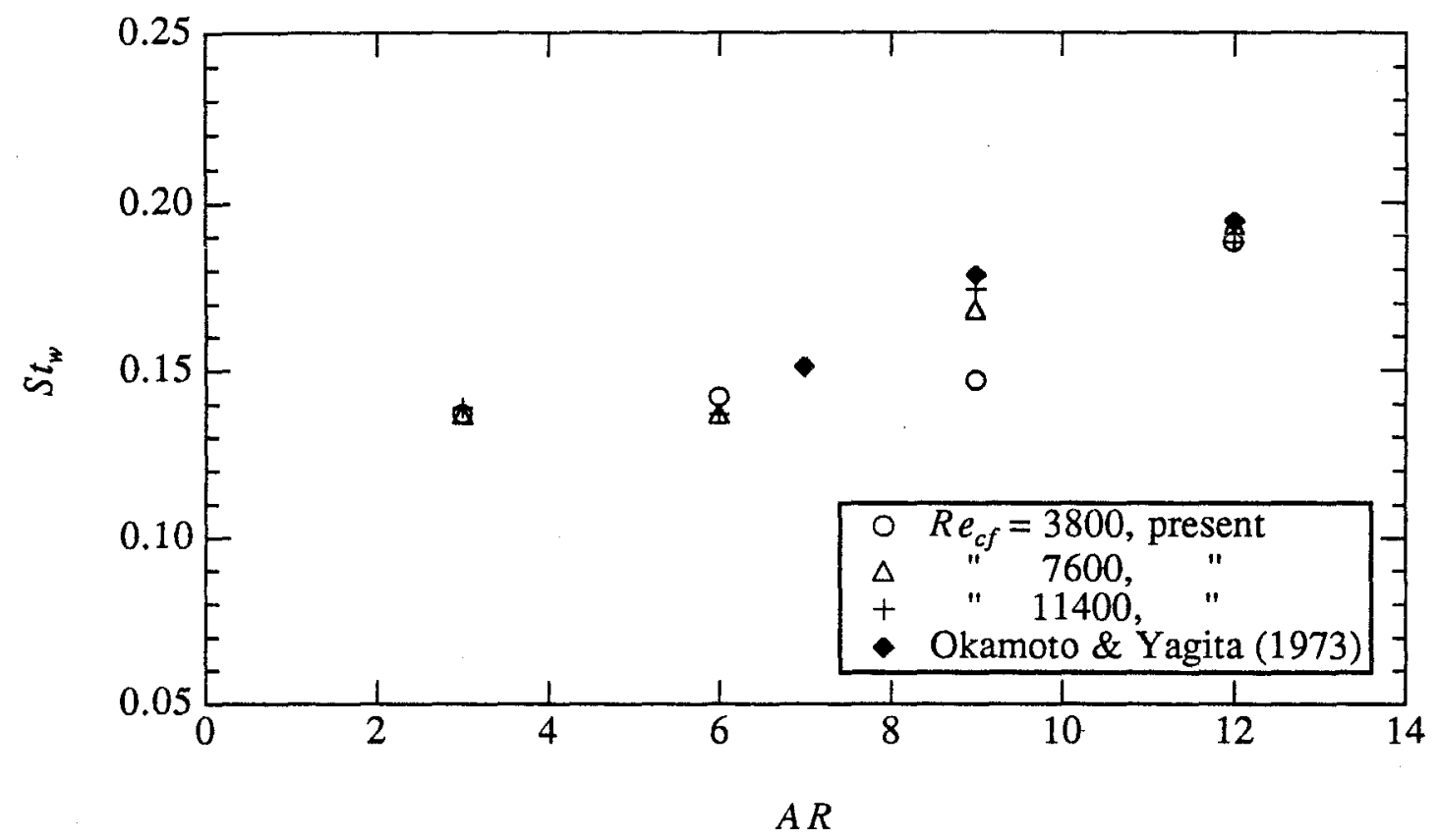

FIGURE 5.35: Wake Strouhal numbers of wall-mounted circular cylinders as a function of aspect ratio. 

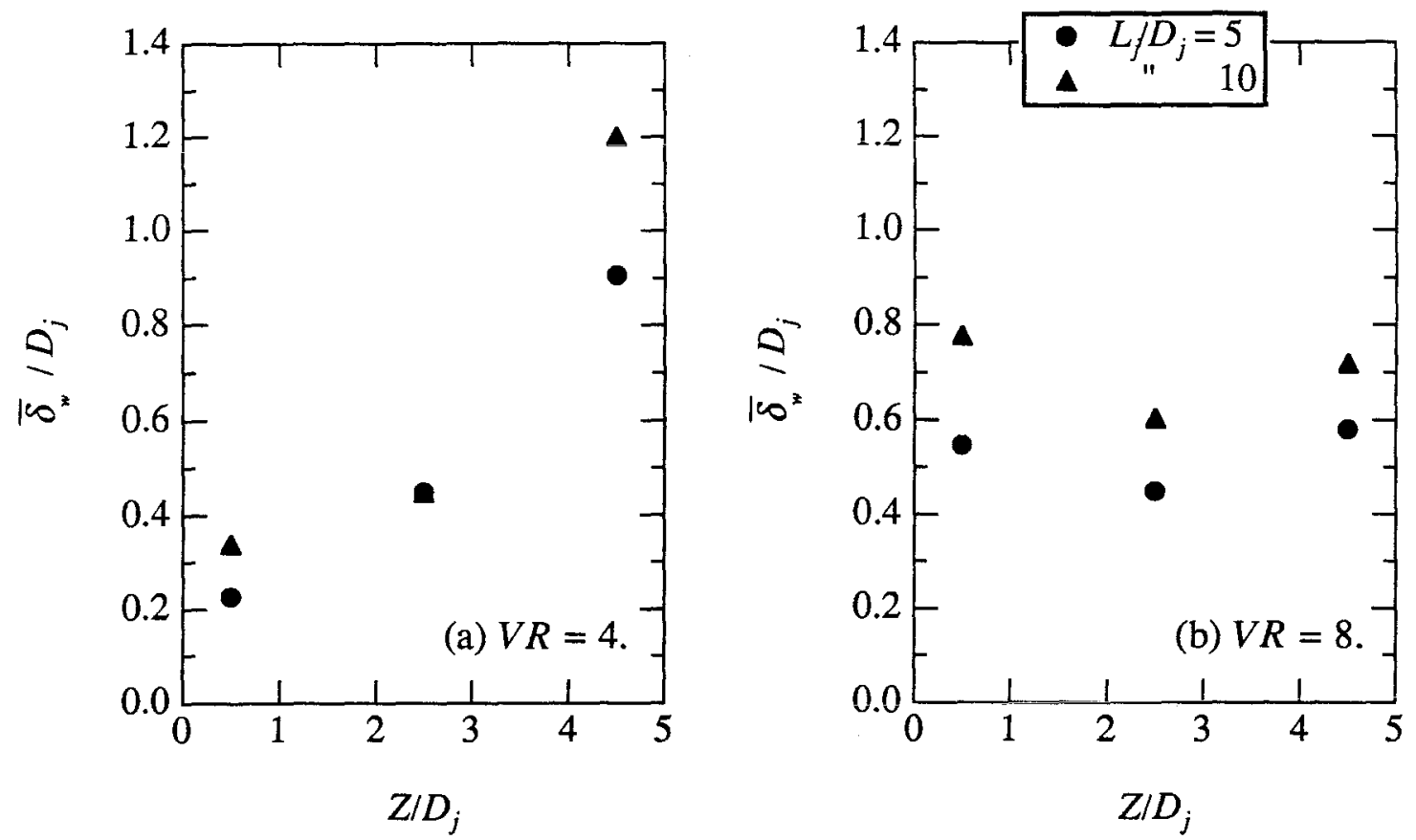

FIGURE 5.36: Dependence of $\bar{\delta}_{w} / D_{j}$ on distance from the crossflow wall.

(a) $V R=4$, (b) $V R=8$.
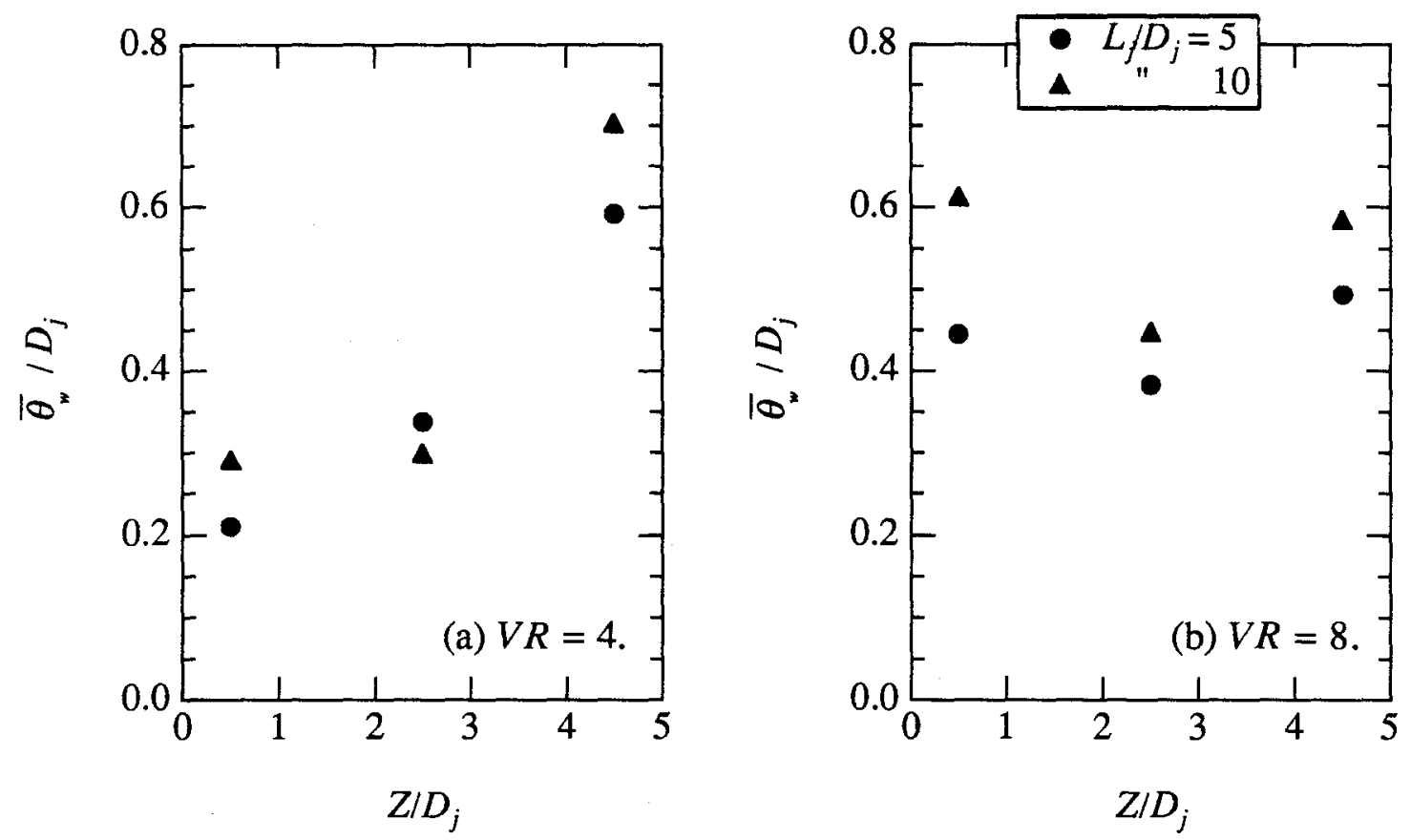

FIGURE 5.37: Dependence of $\bar{\theta}_{w} / D_{j}$ on distance from the crossflow wall. (a) $V R=4$, (b) $V R=8$. 
193
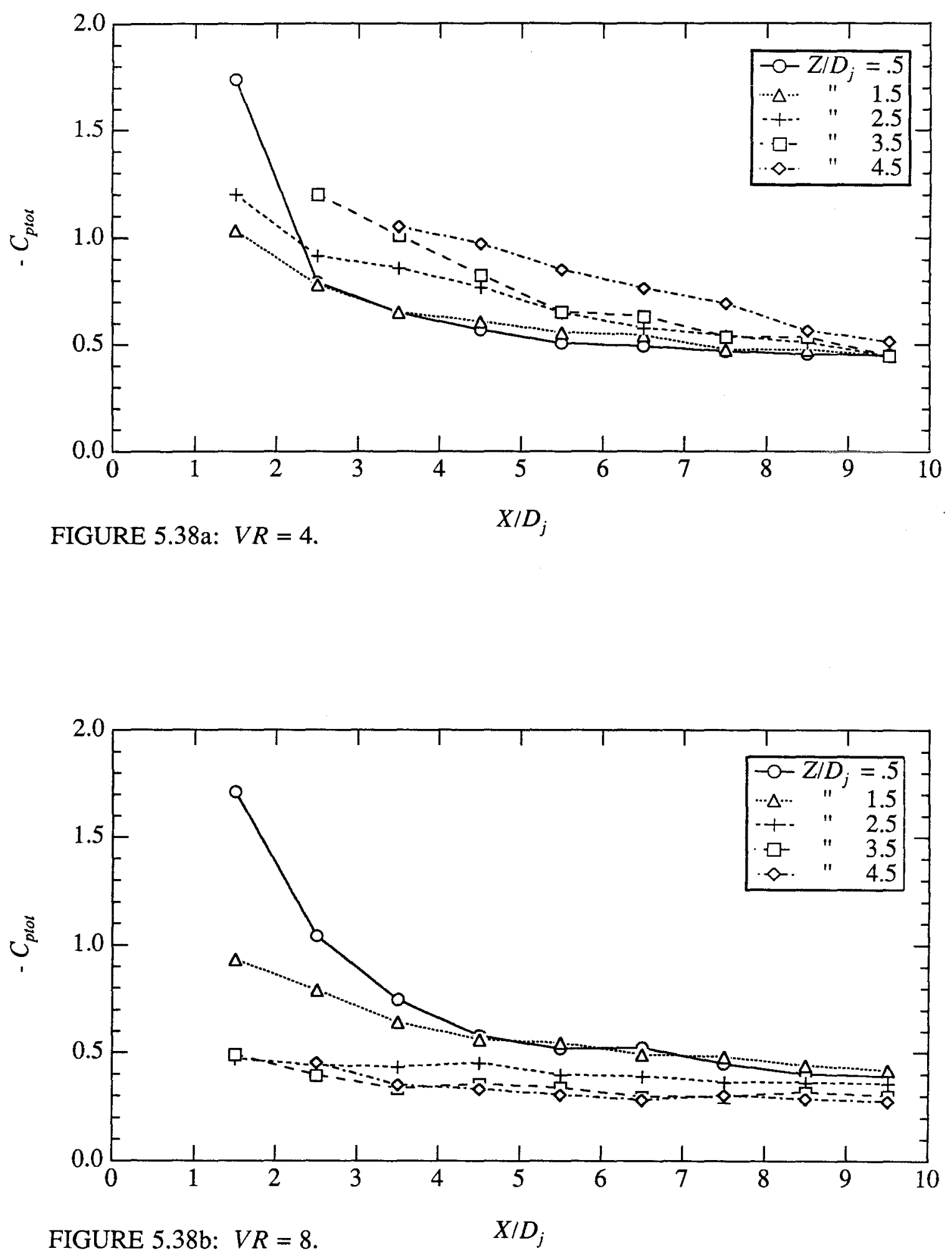

FIGURE 5.38: Wake total pressure deficits at $Y / D_{j}=0$. (a) $V R=4$, (b) $V R=8$, (c) CYLINDER. $R e_{c f}=3800 . L_{j}^{\prime} D_{j}=5$. 


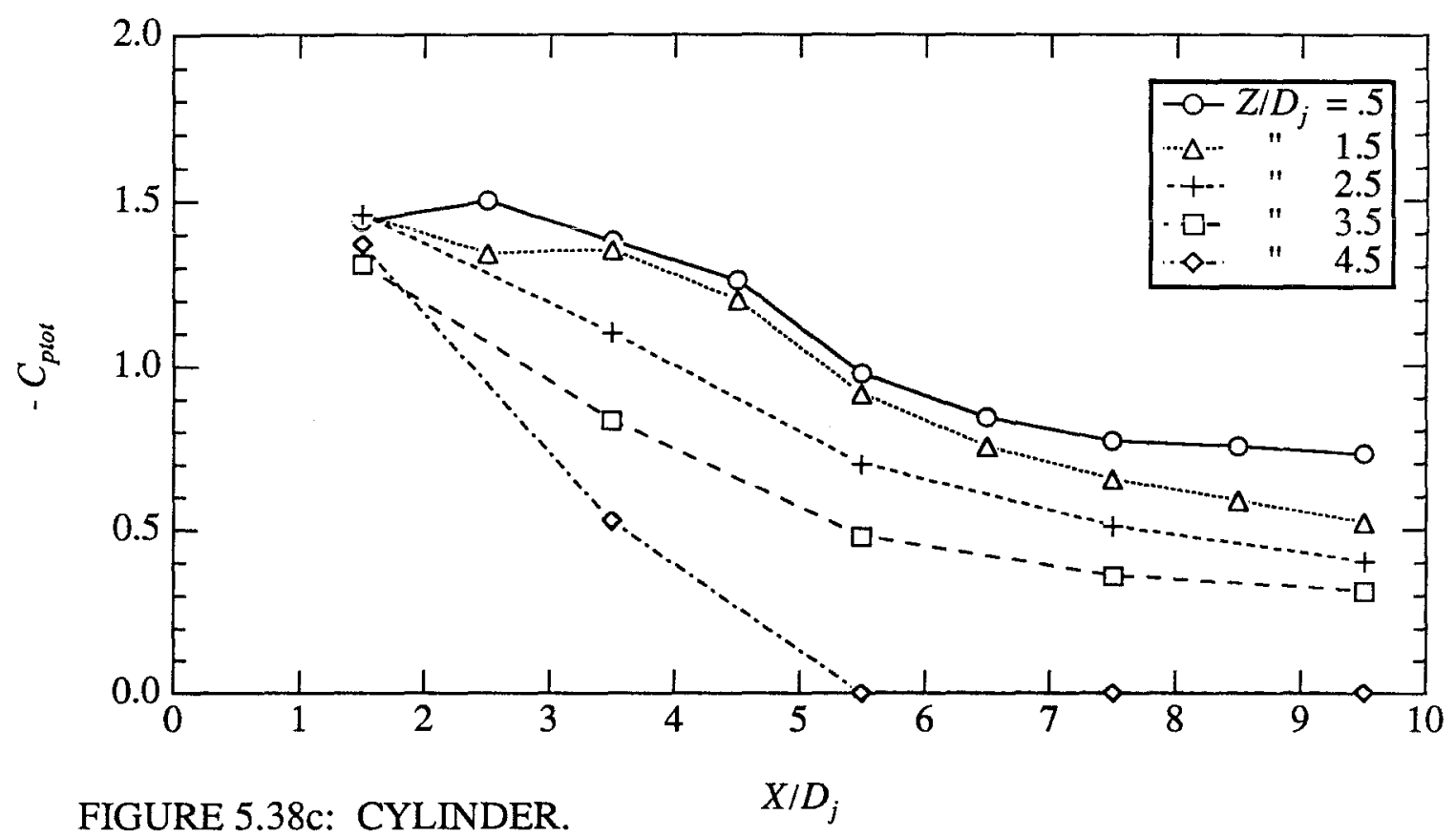




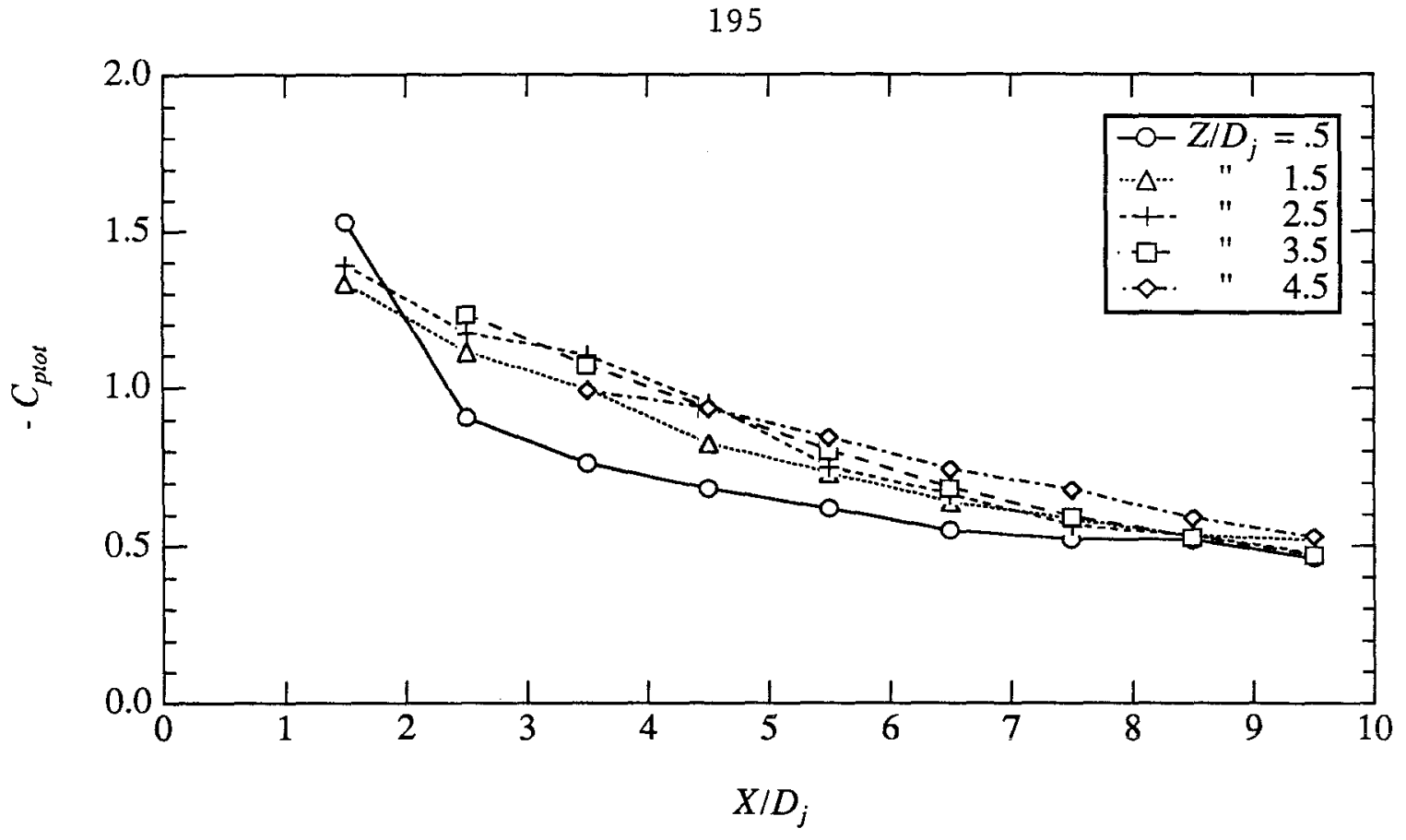

FIGURE 5.39a: $V R=4$.

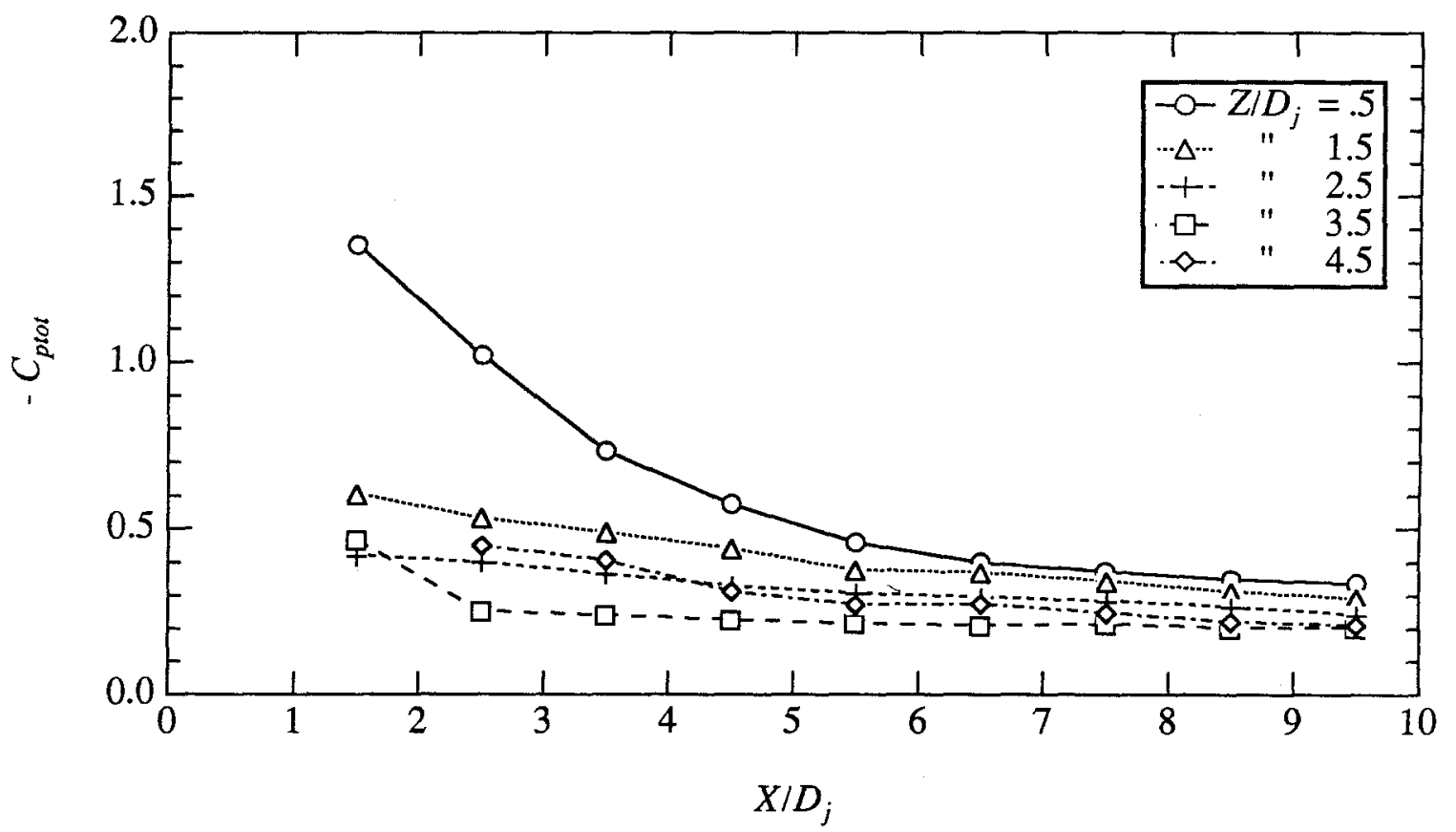

FIGURE 5.39b: $V R=8$.

FIGURE 5.39: Wake total pressure deficits at $Y / D_{j}=0$. (a) $V R=4$, (b) $V R=8$, (c) CYLINDER. $R e_{c f}=7600 . L_{j} / D_{j}=5$. 


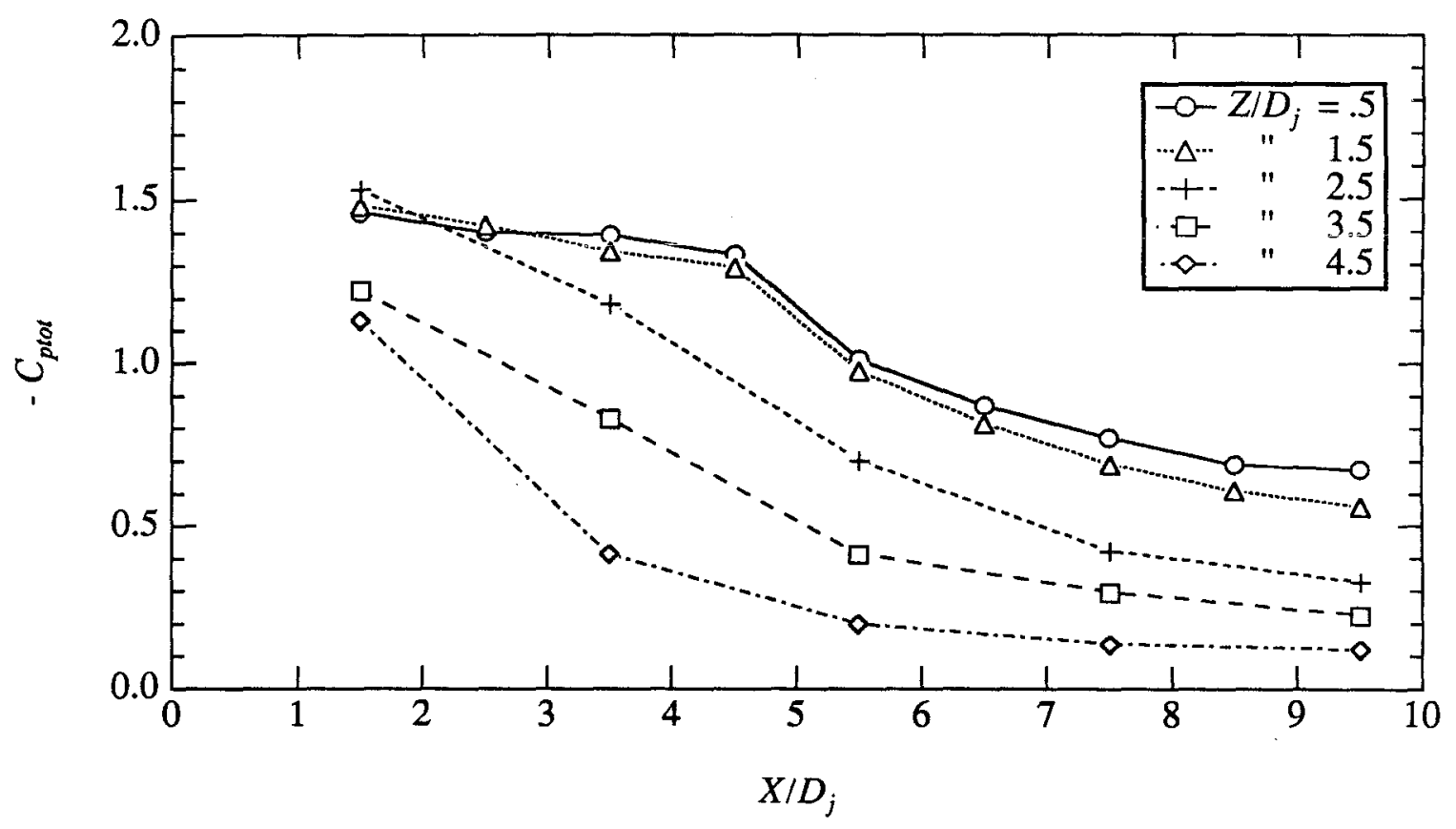

FIGURE 5.39c: CYLINDER. 


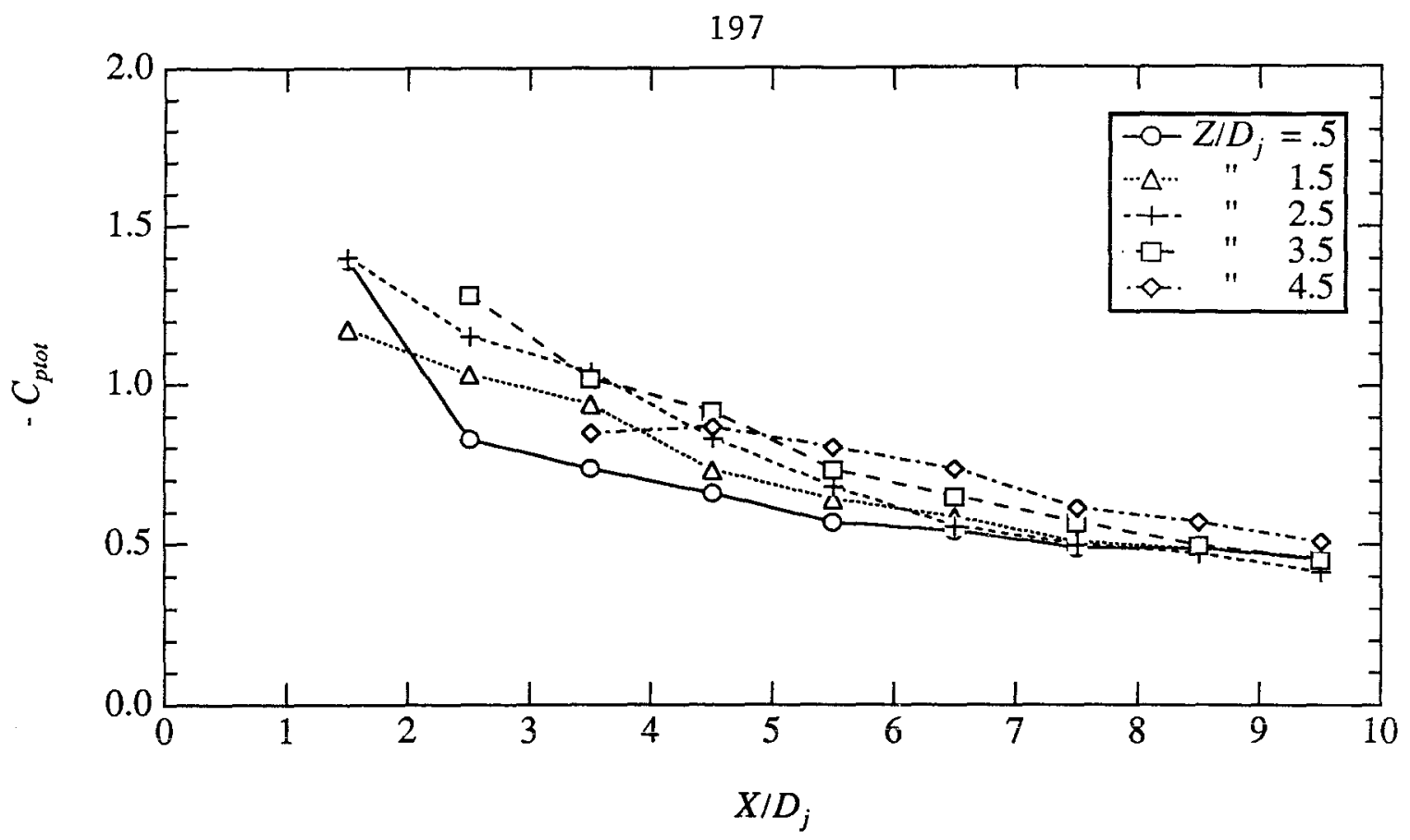

FIGURE 5.40a: $V R=4$

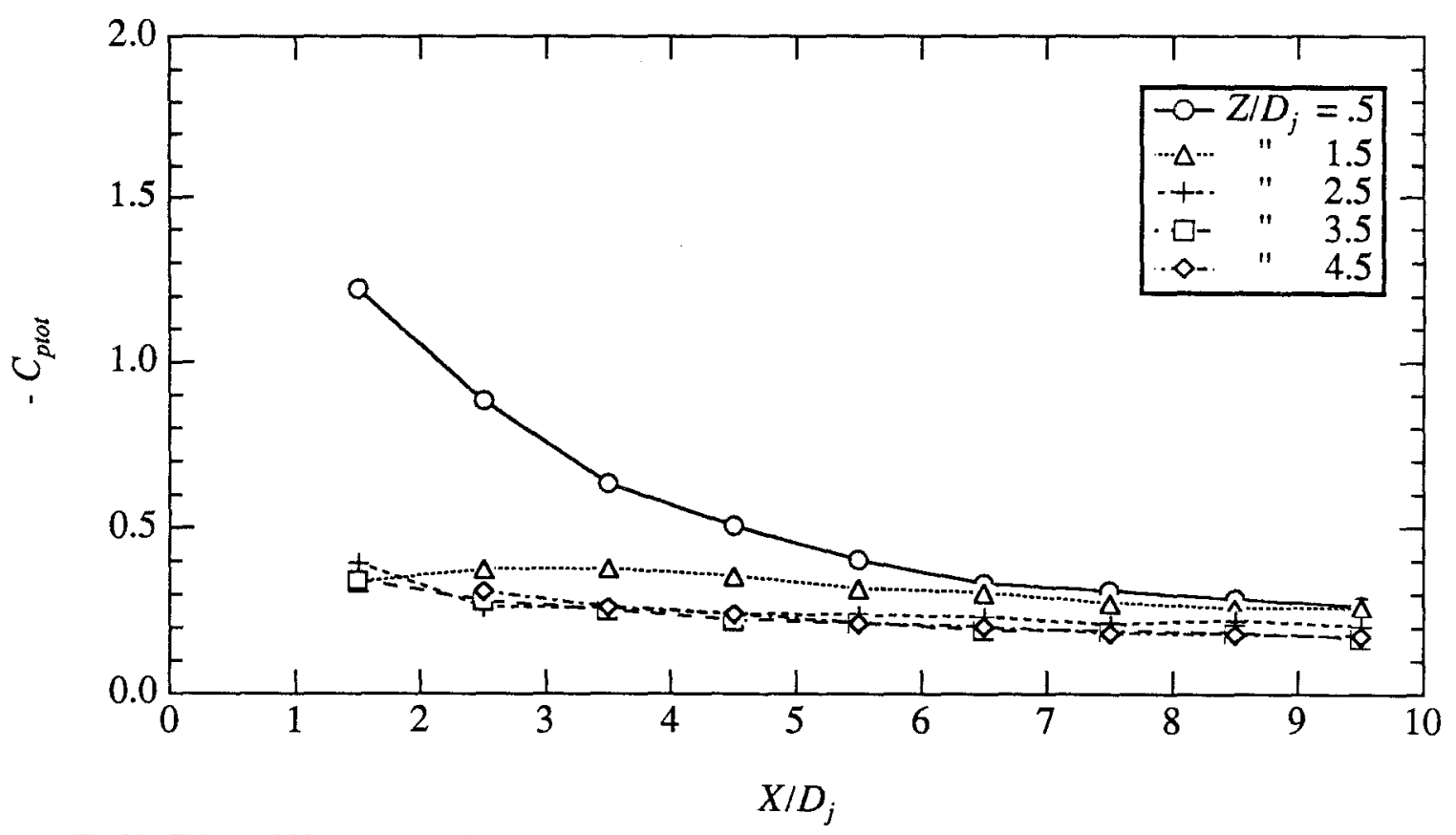

FIGURE 5.40b: $V R=8$.

FIGURE 5.40: Wake total pressure deficits at $Y / D_{j}=0$. (a) $V R=4$, (b) $V R=8$, (c) CYLINDER. $R e_{c f}=11400 . L_{j} / D_{j}=5$. 


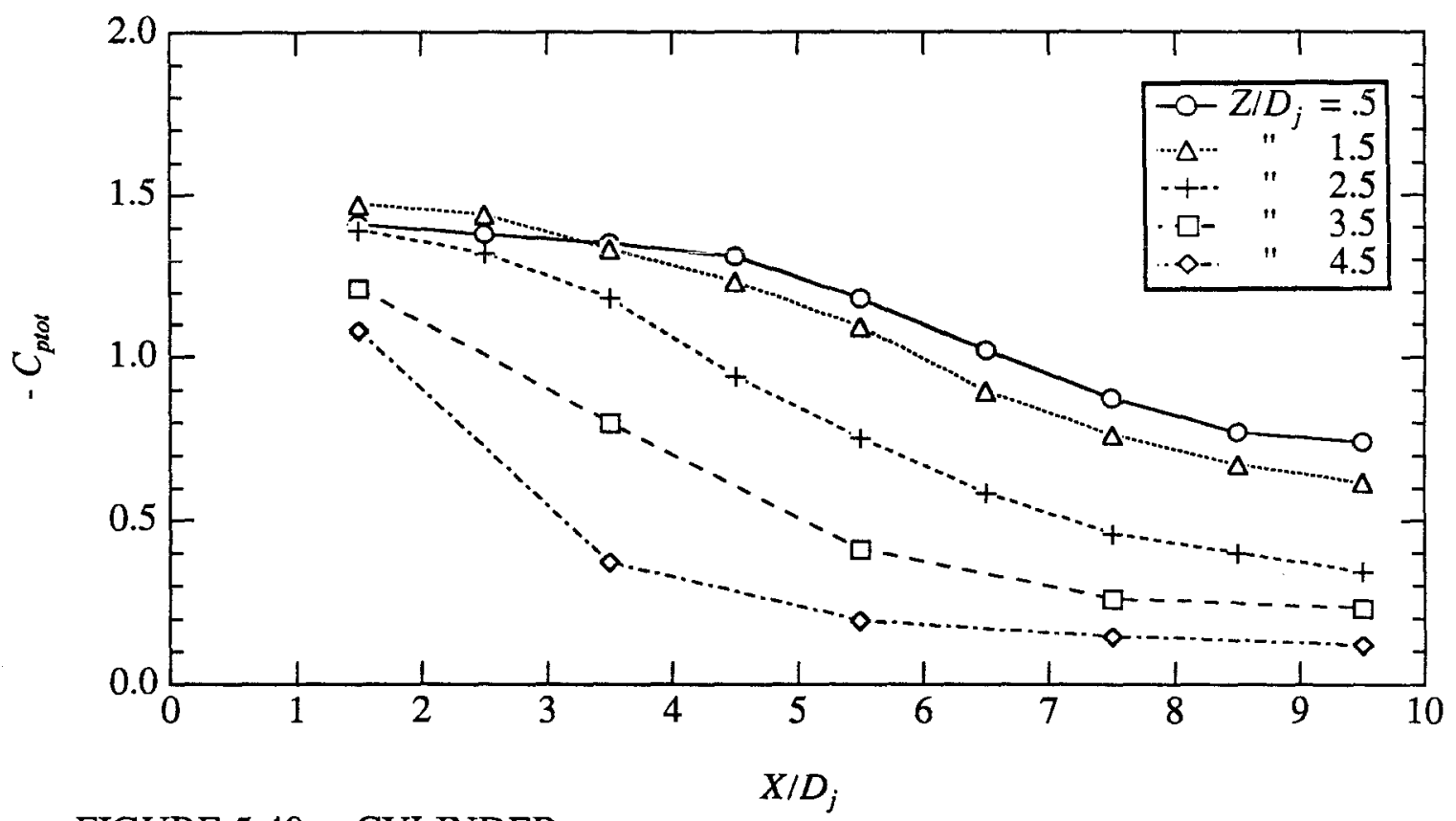

FIGURE 5.40c: CYLINDER. 


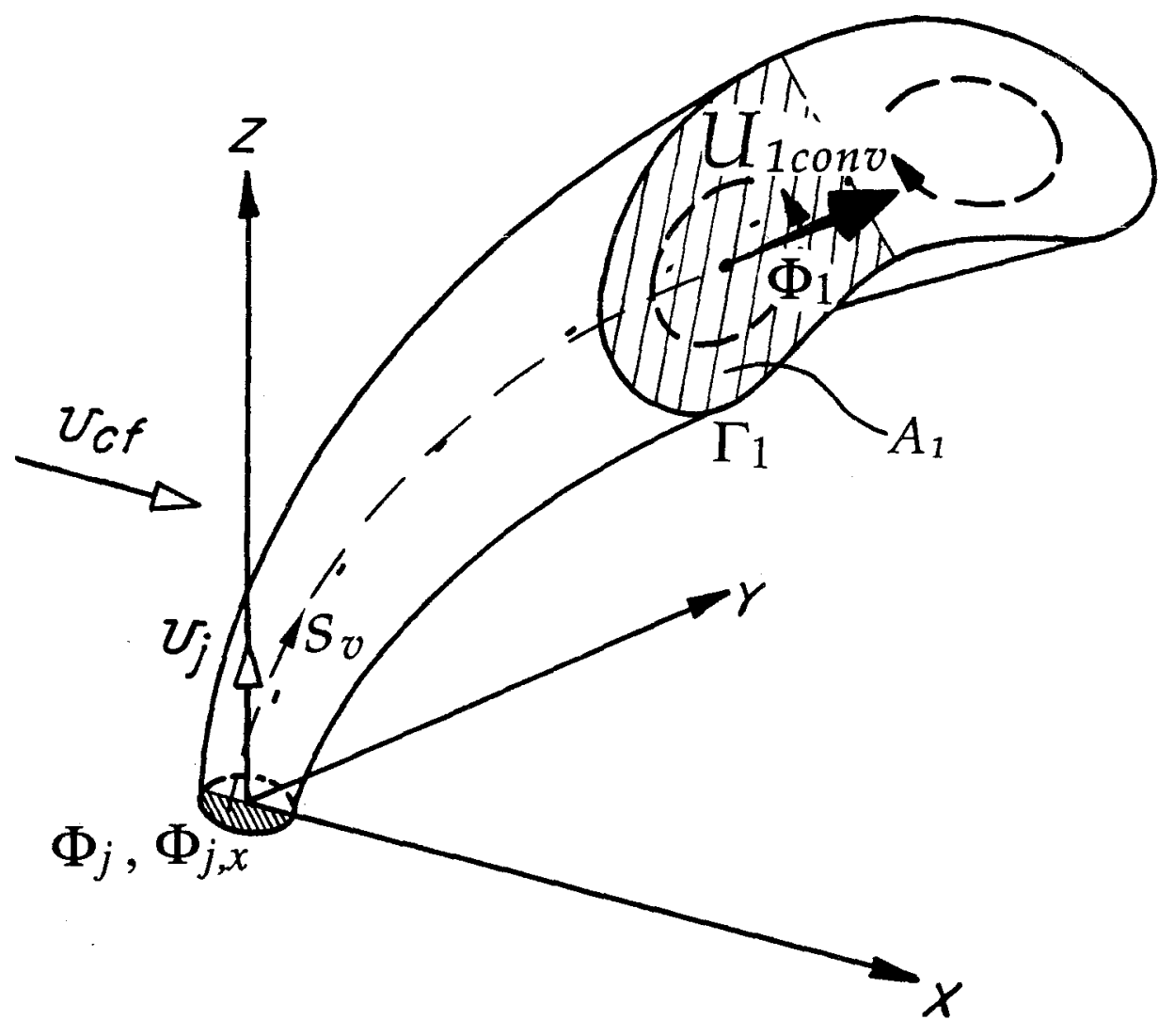

FIGURE 6.1: Sketch of the deflected jet with notation highlighting one vortex of the CVP. 


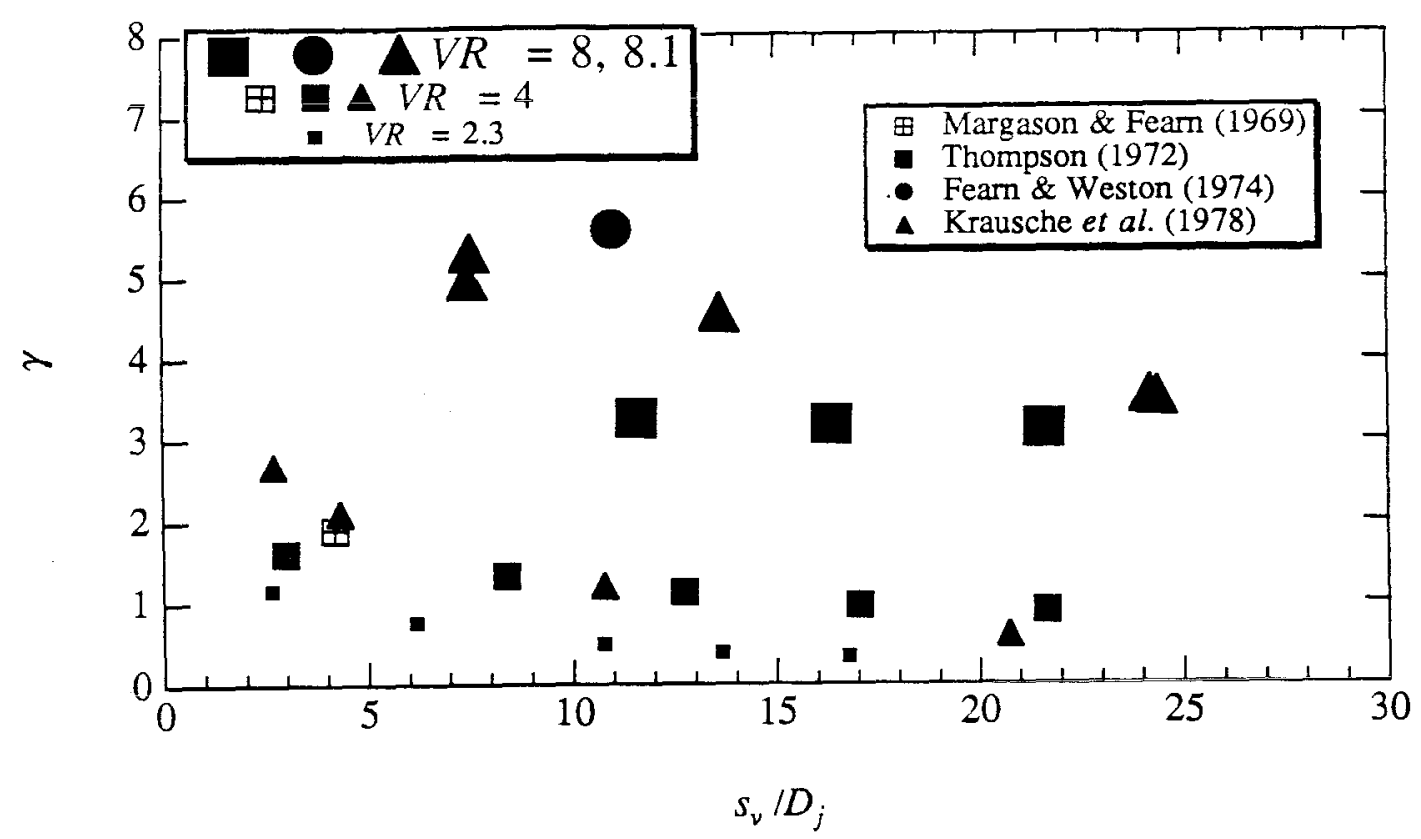

FIGURE 6.2: Experimental results for the nondimensional circulation of one CVP vortex along its trajectory.

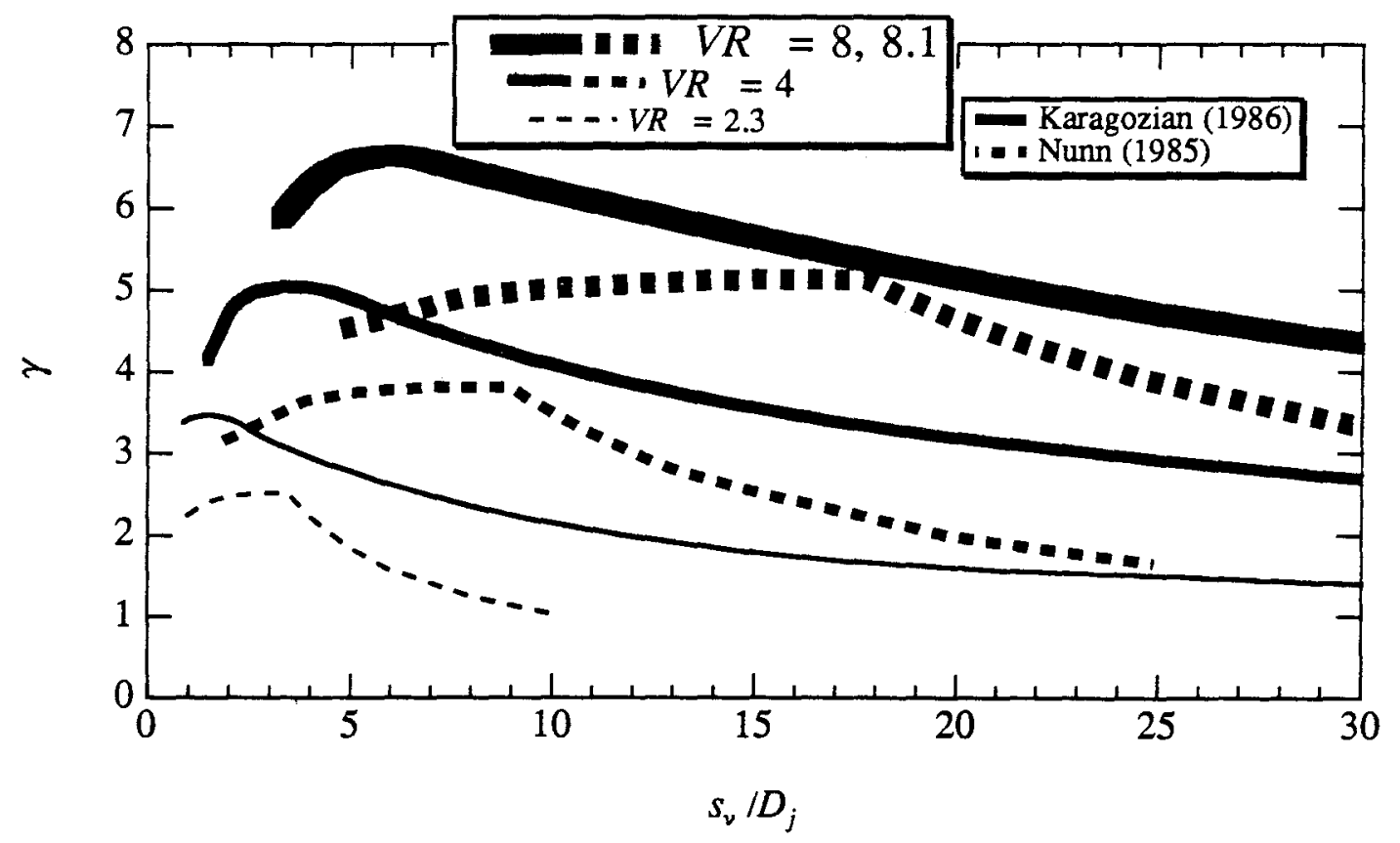

FIGURE 6.3: Analytic results for the nondimensional circulation of one CVP vortex along its trajectory. 


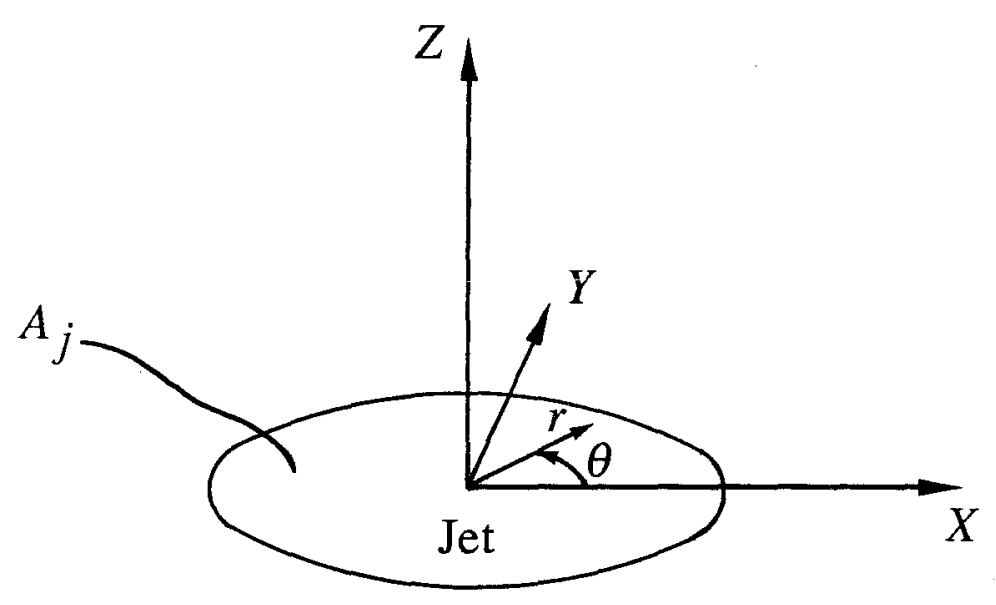

FIGURE 6.4: Notation for vorticity flux estimate at the jet orifice. 


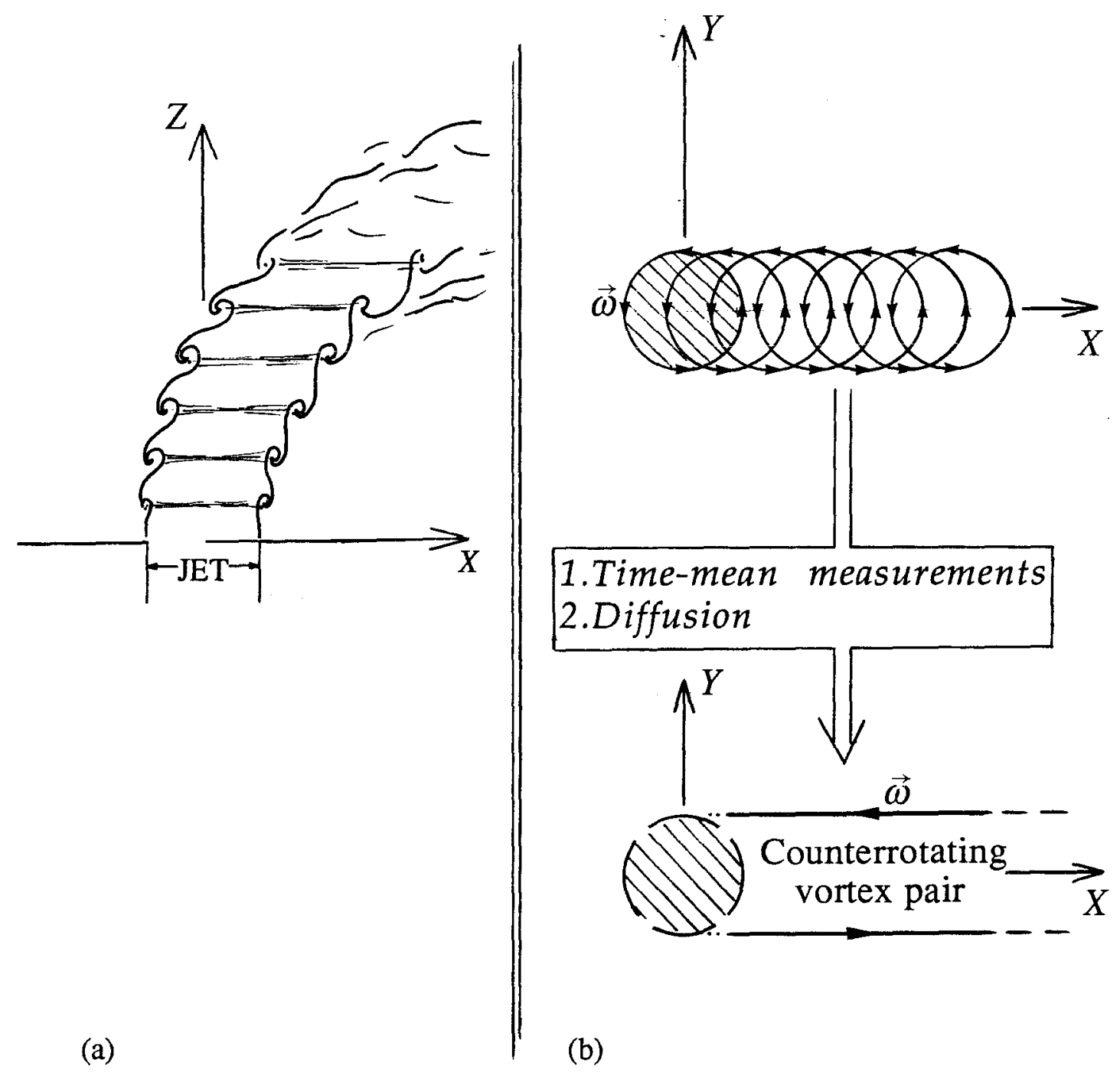

FIGURE 6.5: Idealized model of a jet leading to a counterrotating vortex pair.

(a) Side view, (b) Projection onto a constant $Z$ plane. 
203

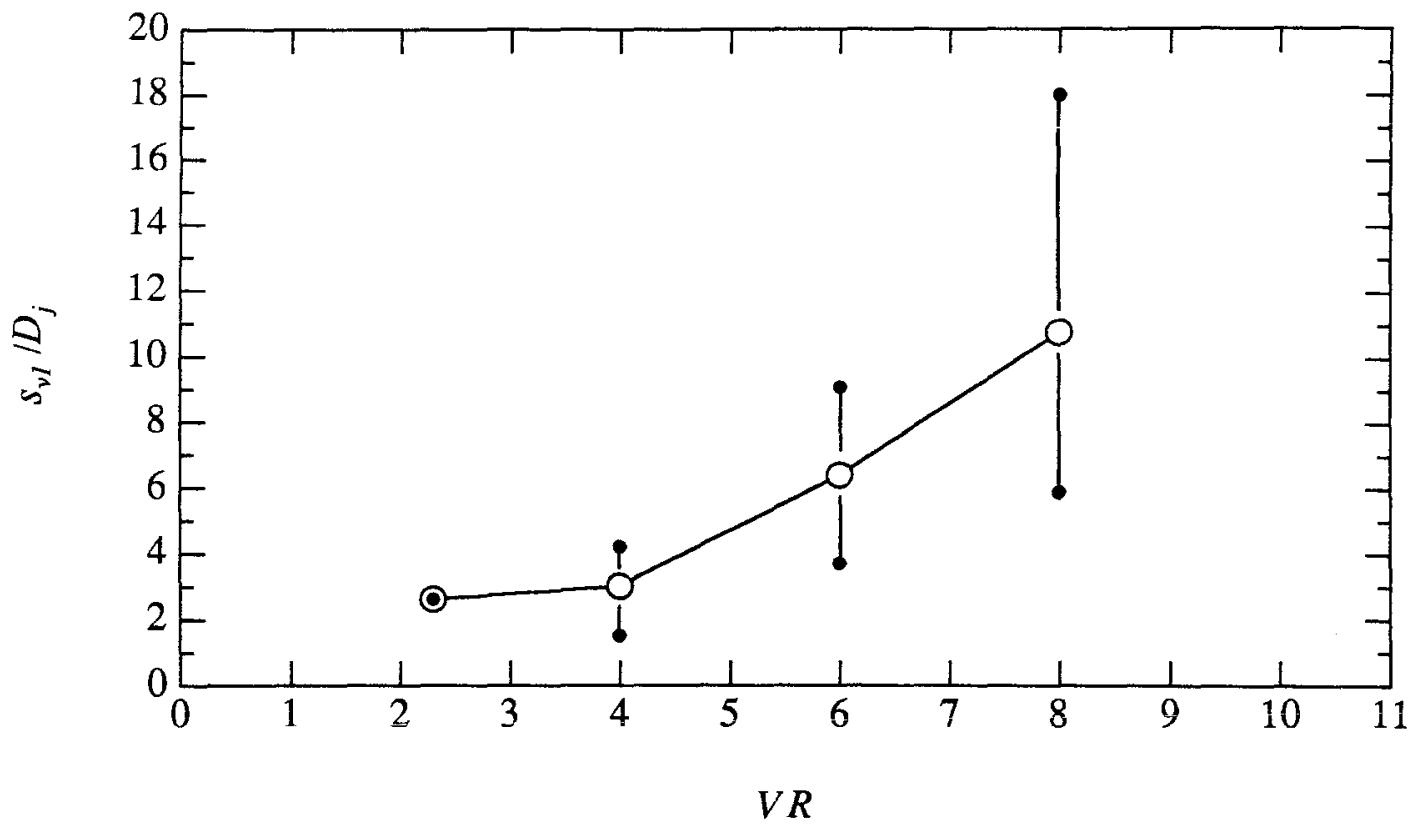

FIGURE 6.6: $s_{v I} / D_{j}$ vs. $V R$. Open circles indicate averages from figures 6.2 and 6.3 references. Solid points show the ranges of disagreement among those results.

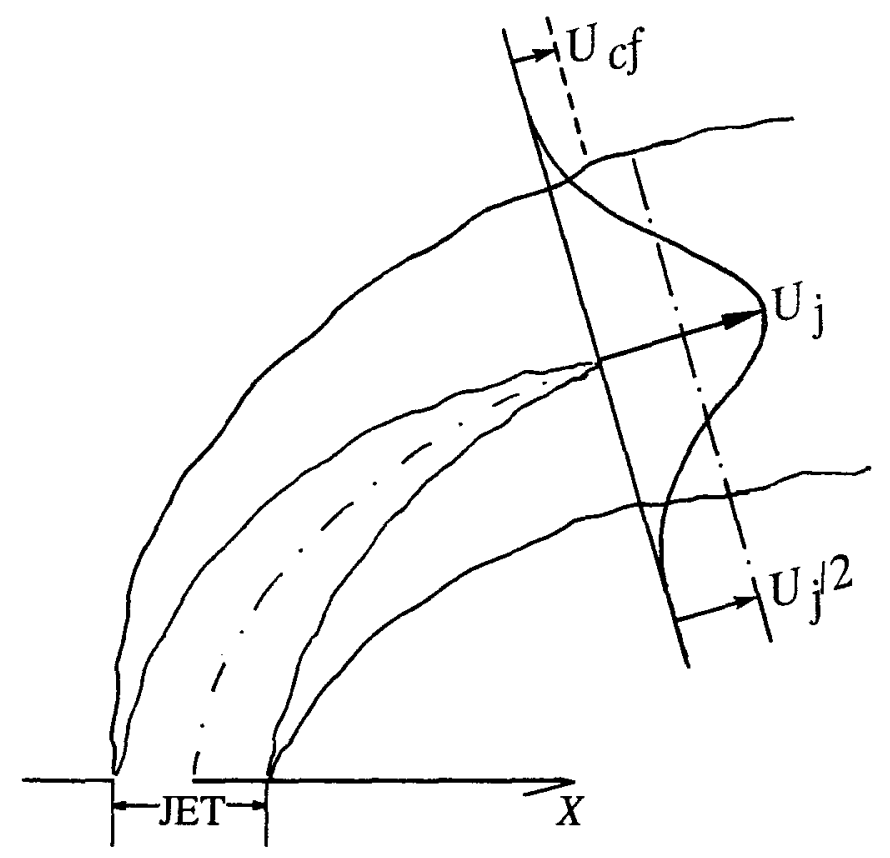

FIGURE 6.7: View of deflected jet used to estimate the convective velocity at the point of CVP vortex full-development. 


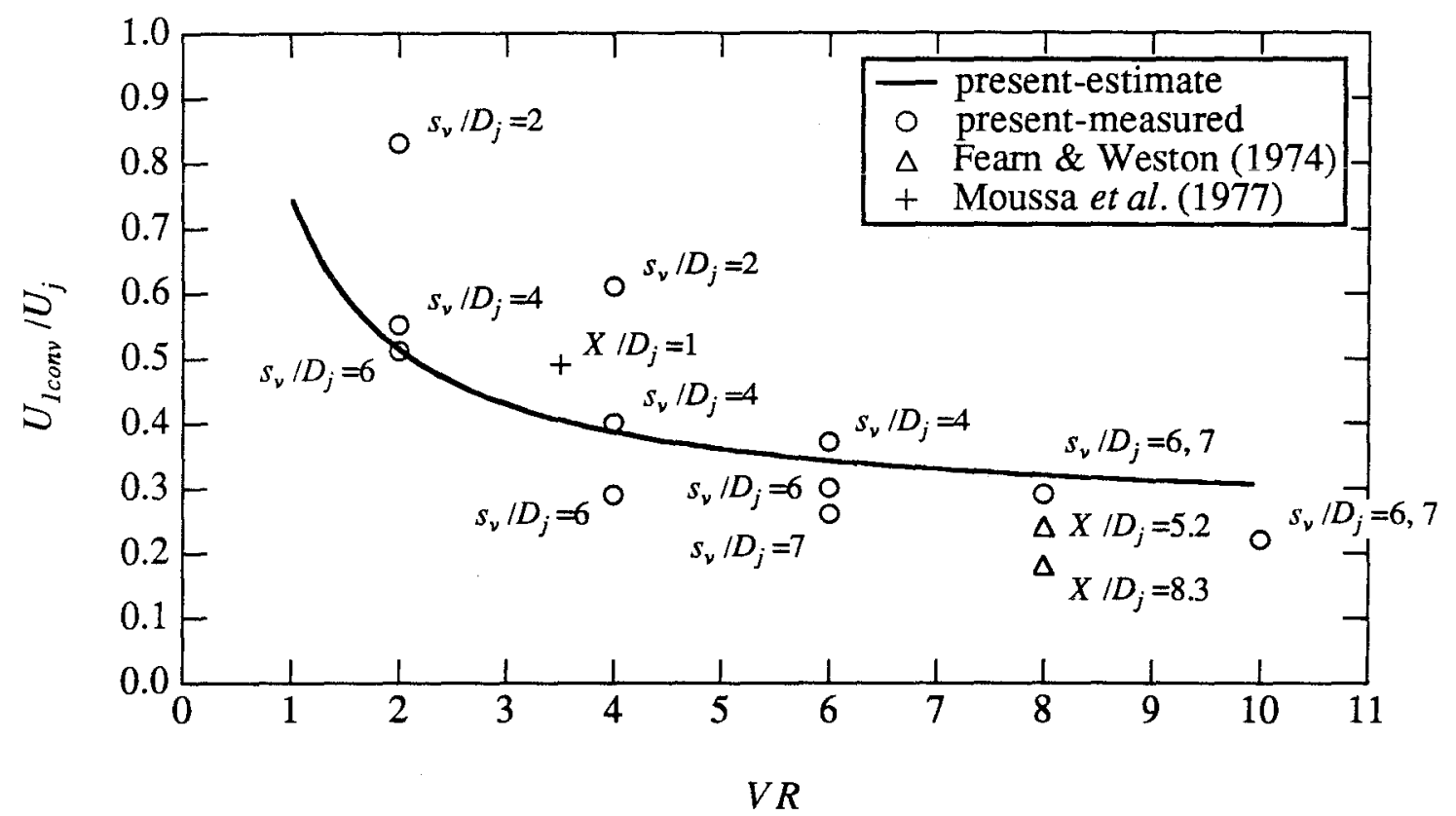

FIGURE 6.8: Convective velocities. Estimates from equation 6.16 and experimental results. Locations of measurements are indicated next to the data. 


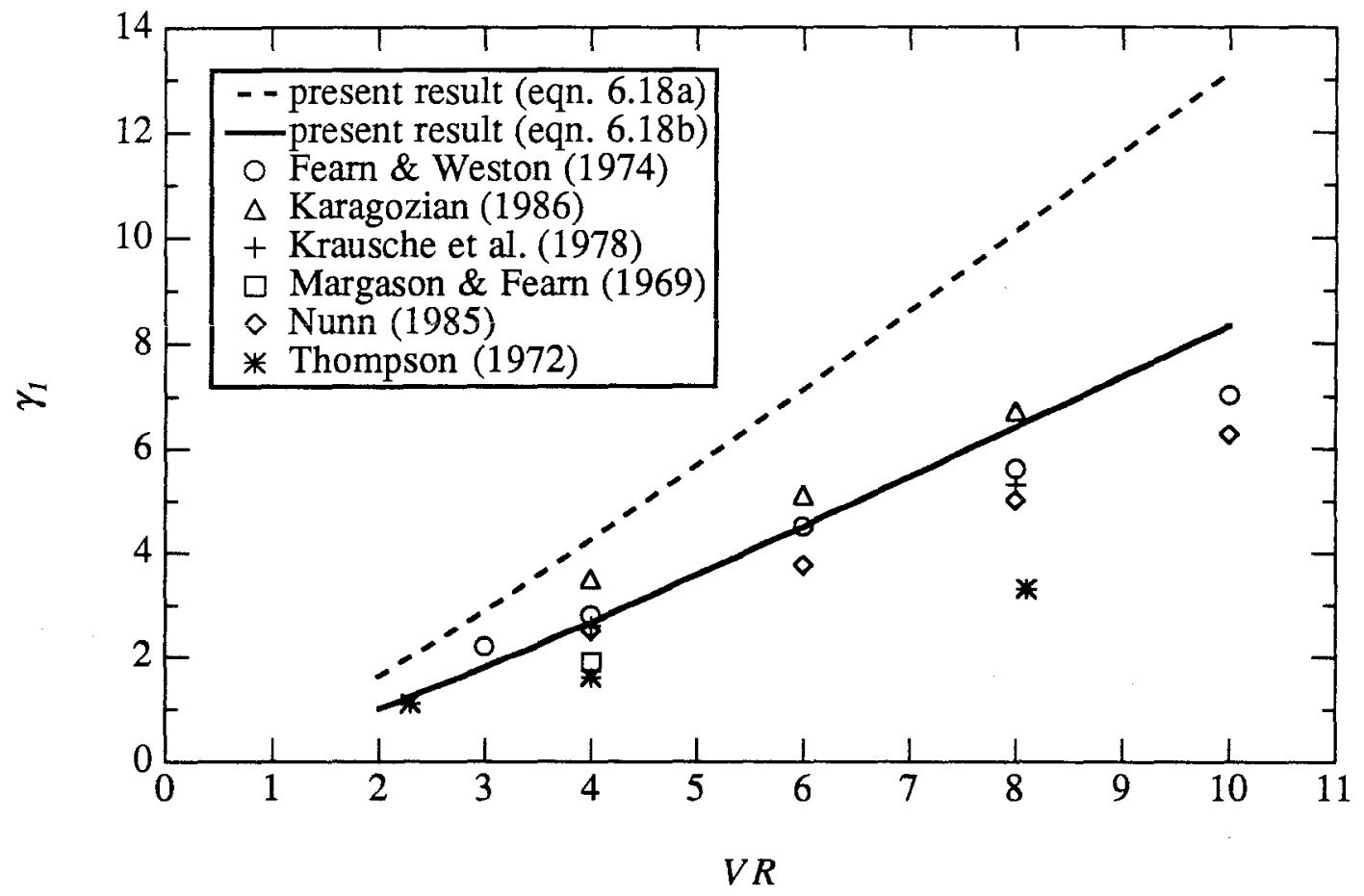

FIGURE 6.9: Comparison of the estimated circulation of a CVP vortex with the results of others. 


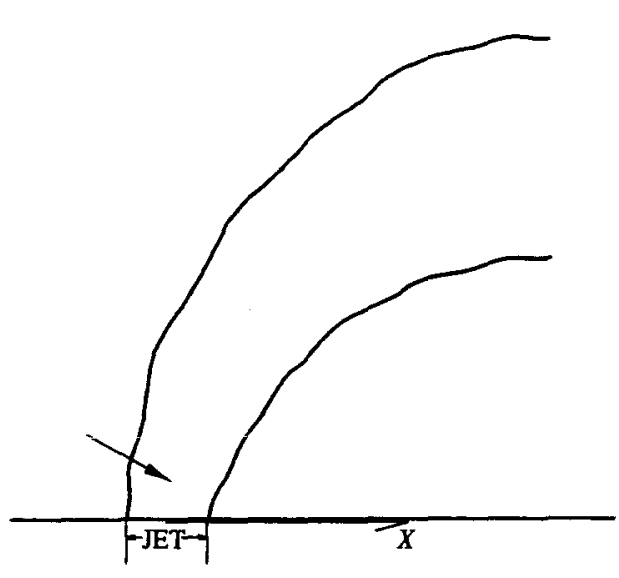

Side View

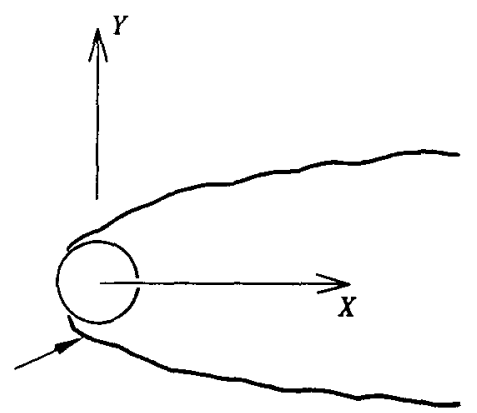

Top View

FIGURE 7.1: A location of $\zeta$, the $Z$ component of vorticity, is indicated by the arrows.

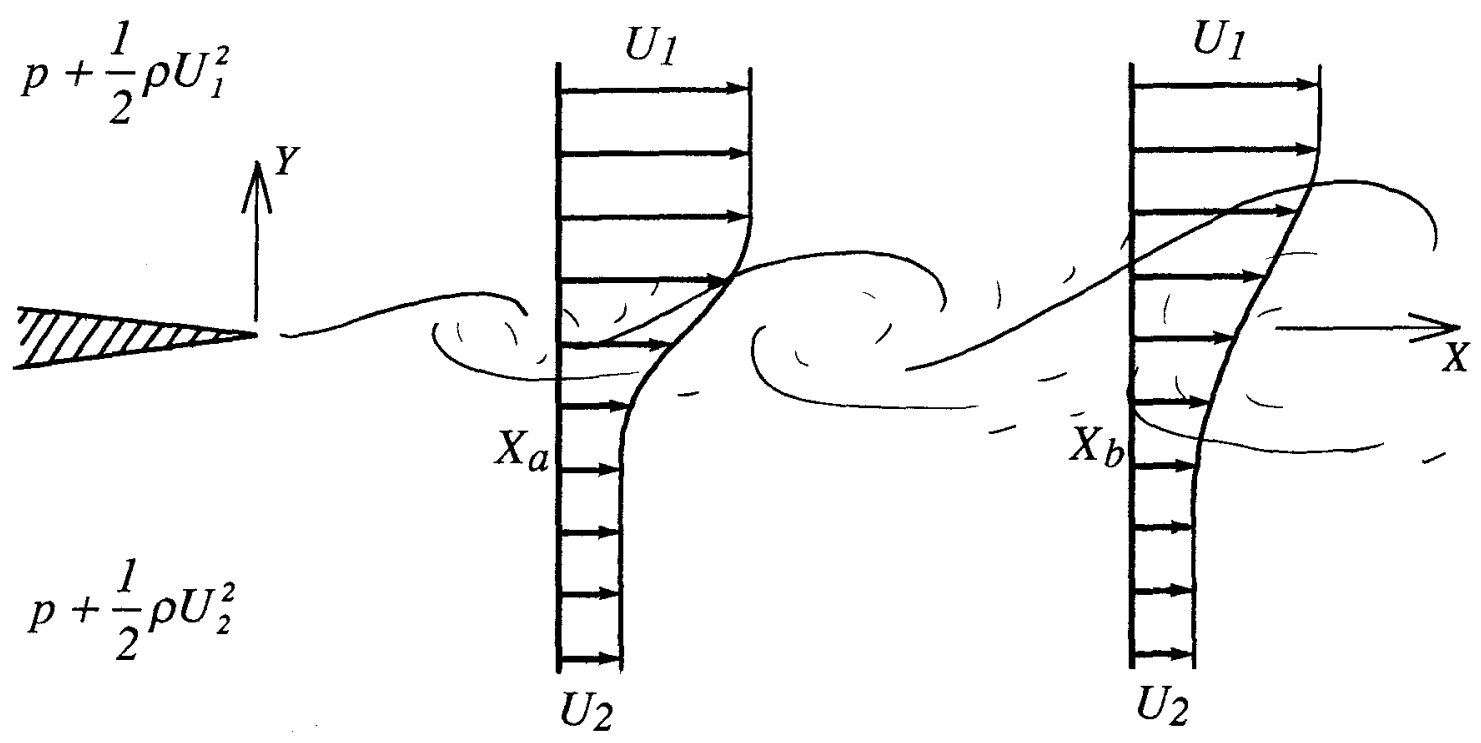

FIGURE 7.2: Schematic of the total pressure gradient across a plane shear layer. 

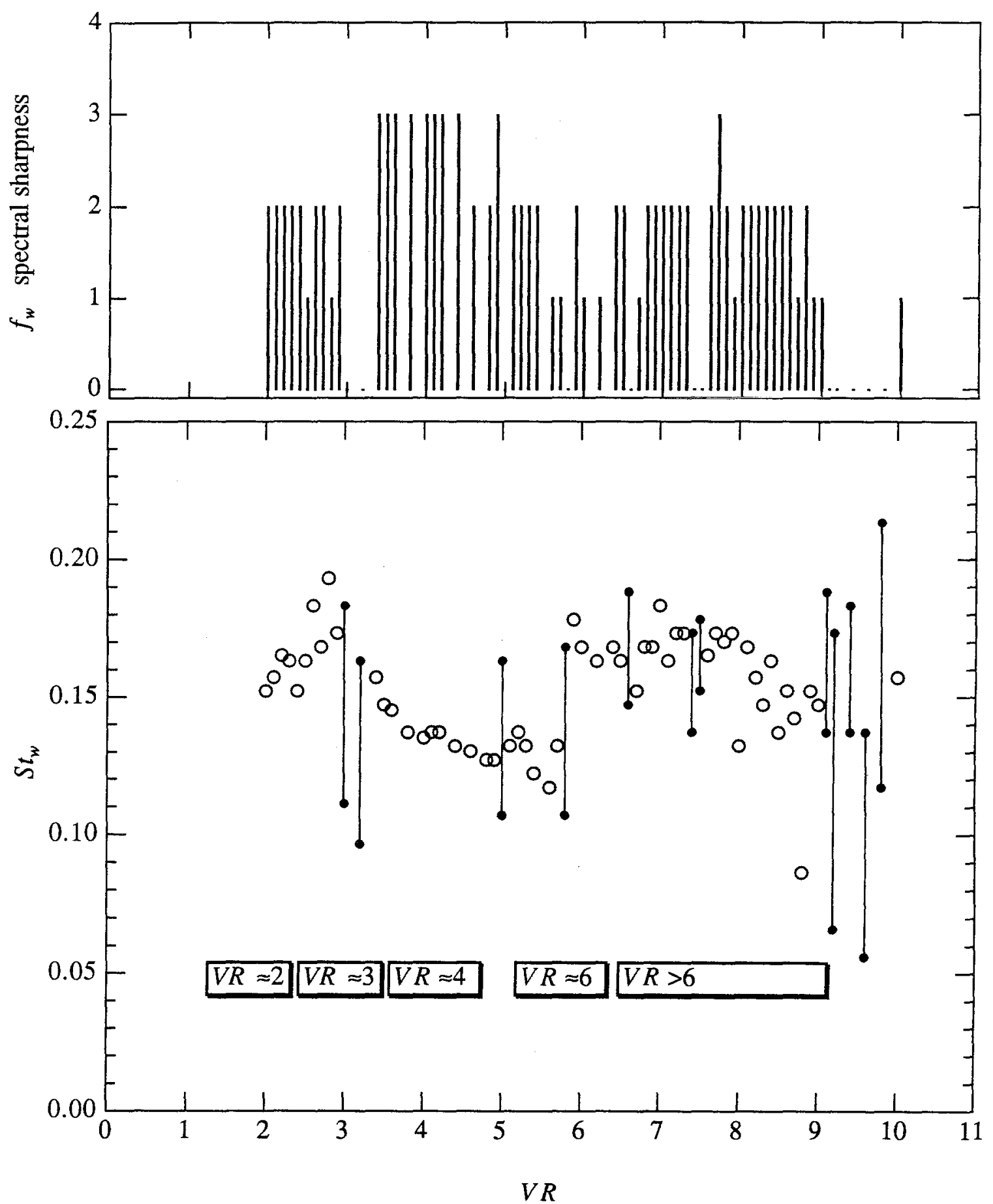

FIGURE 7.3: Wake Strouhal numbers with five $V R$ regimes indicated. $R e_{c f}=3800$. $L_{j} / D_{j}=5$. 

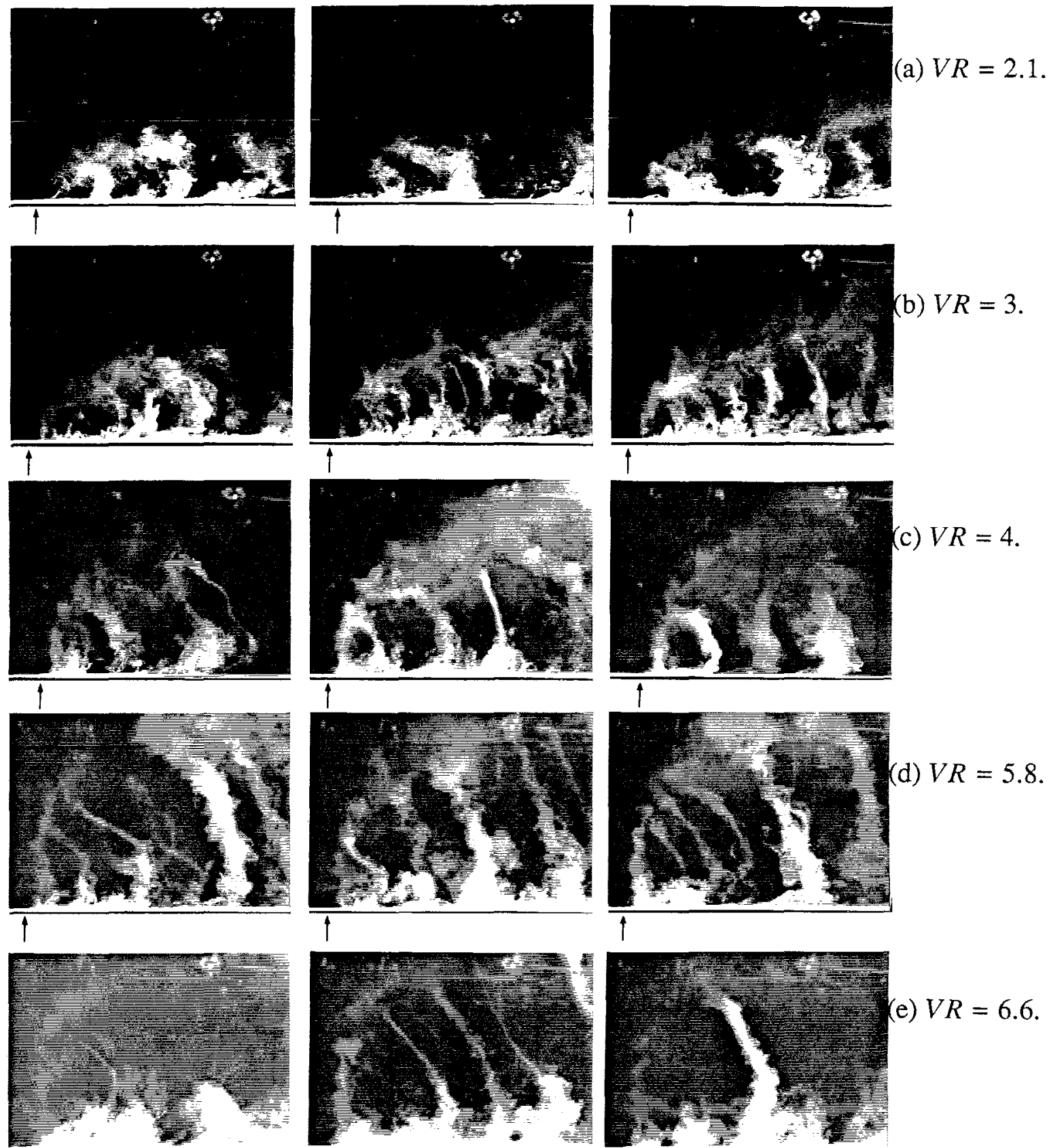

c) $V R=4$
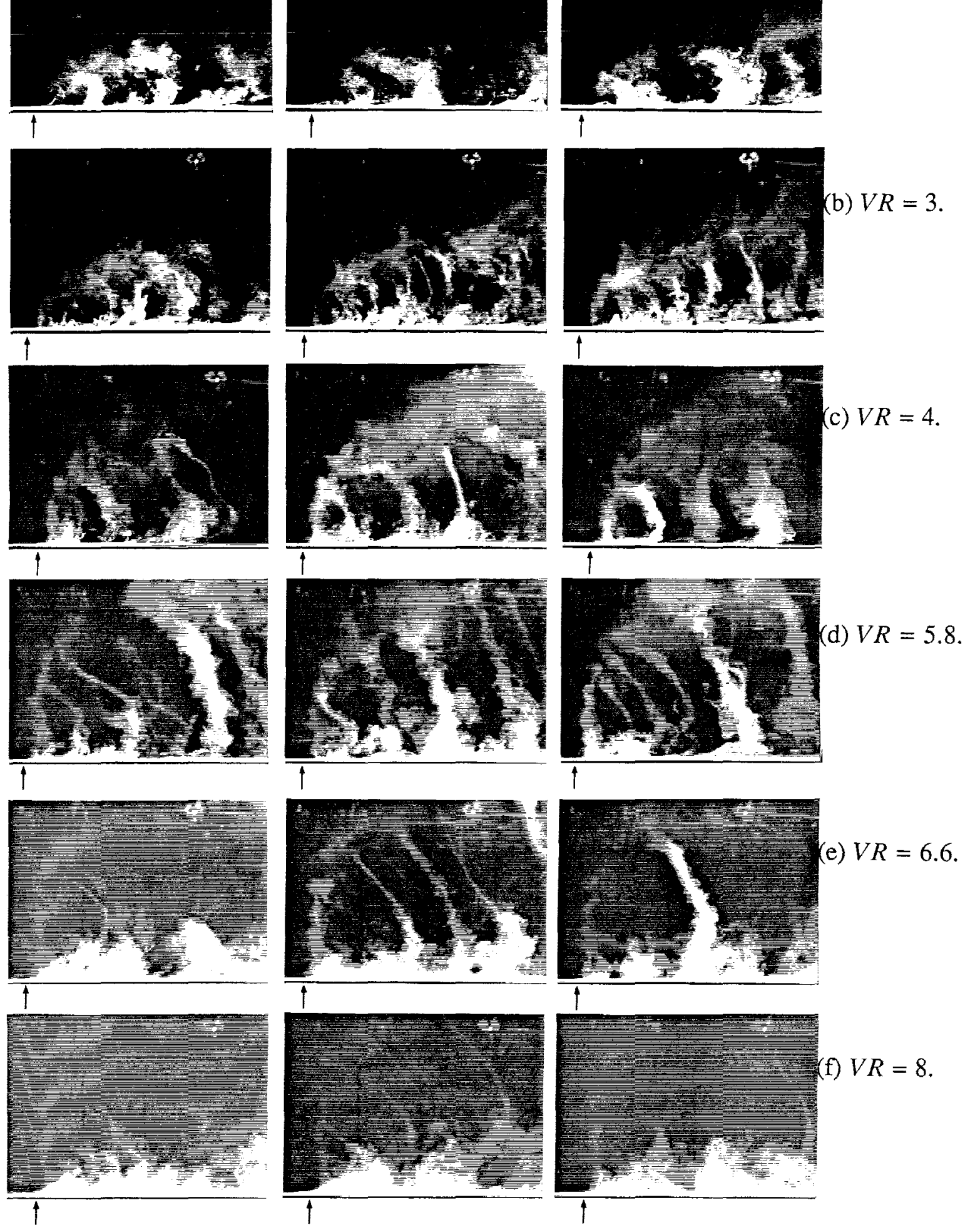

FIGURE 7.4: Typical wake side views. The arrow in each photograph indicates the jet location. (a) $V R=2.1$, (b) $V R=3$, (c) $V R=4$, (d) $V R=5.8$, (e) $V R=6.6$, (f) $V R=8 . R e_{c f}=3800 . Z_{s w} / D_{j}=0+$. 

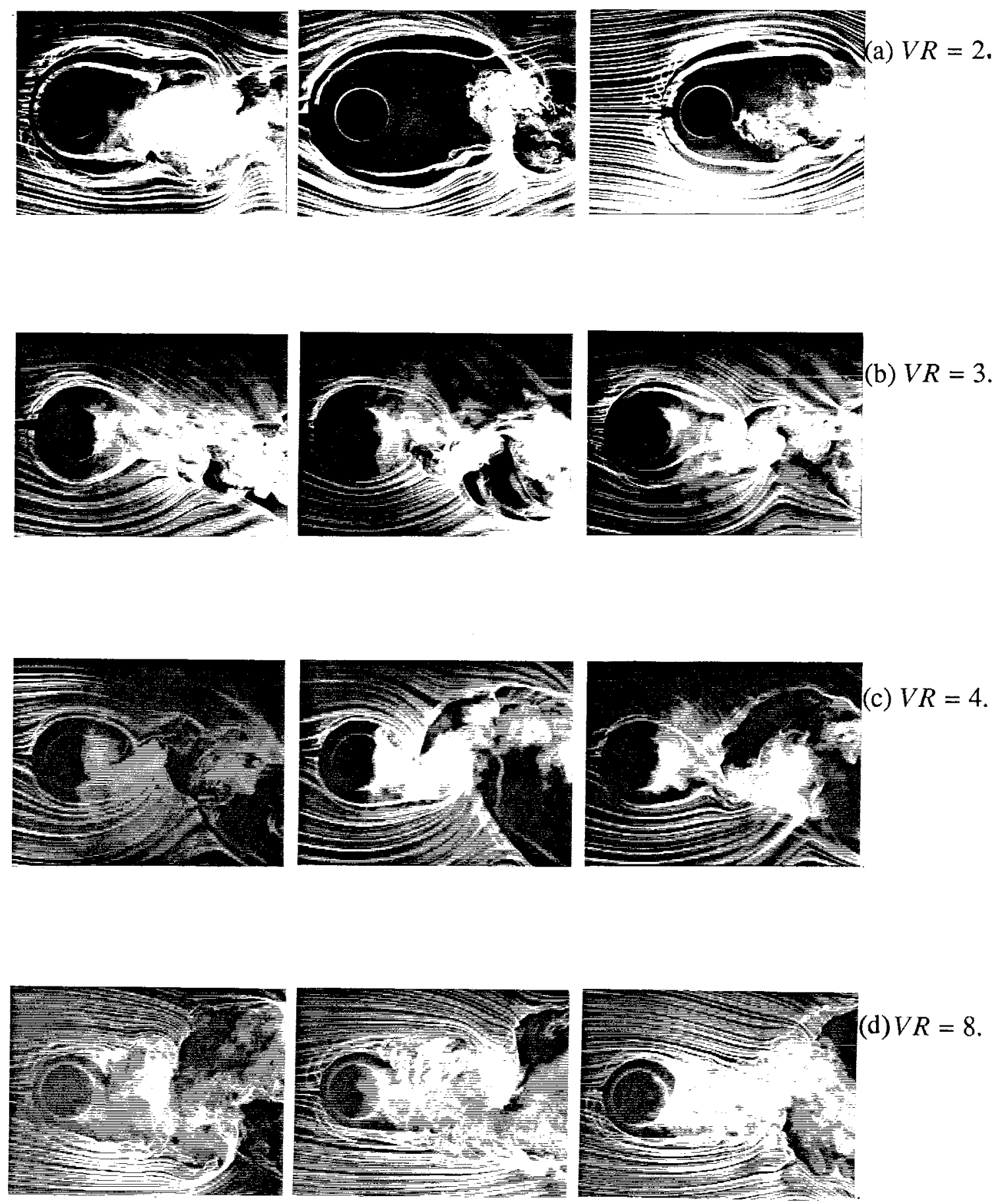

FIGURE 7.5: Typical views of the near wall, very near wake. (a) $V R=2$, (b) $V R=3$, (c) $V R=4$, (d) $V R=8 . R e_{c f}=3800 . Z_{s w} / D_{j}=0+$. 
210

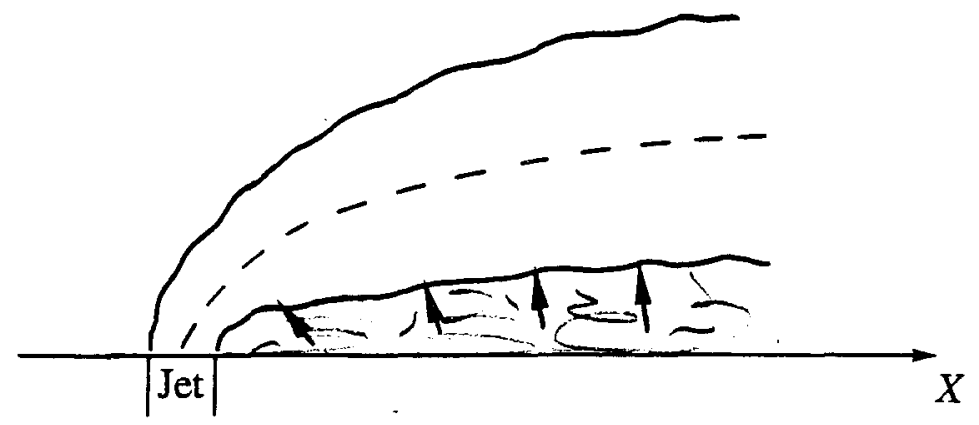

(a) $V R=2$.

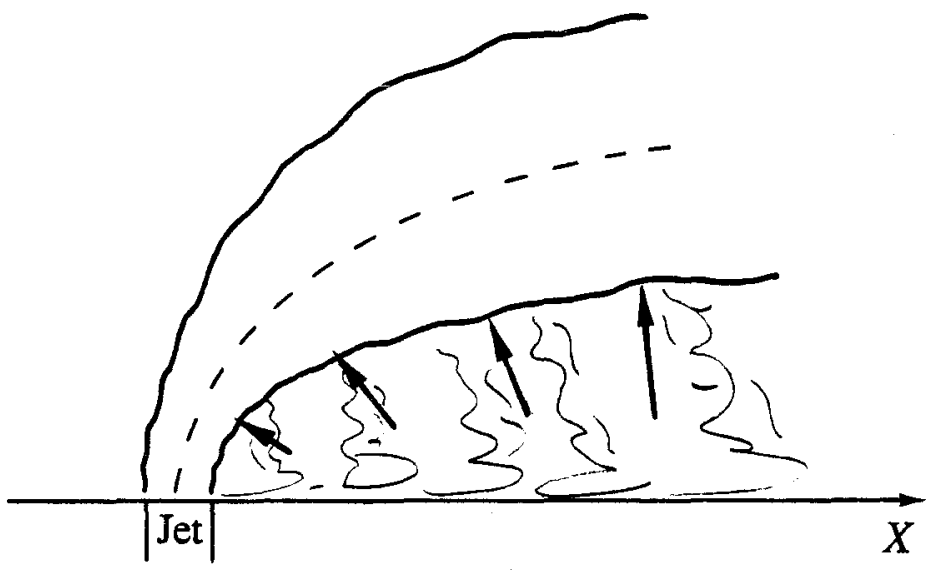

(b) $V R=4$.

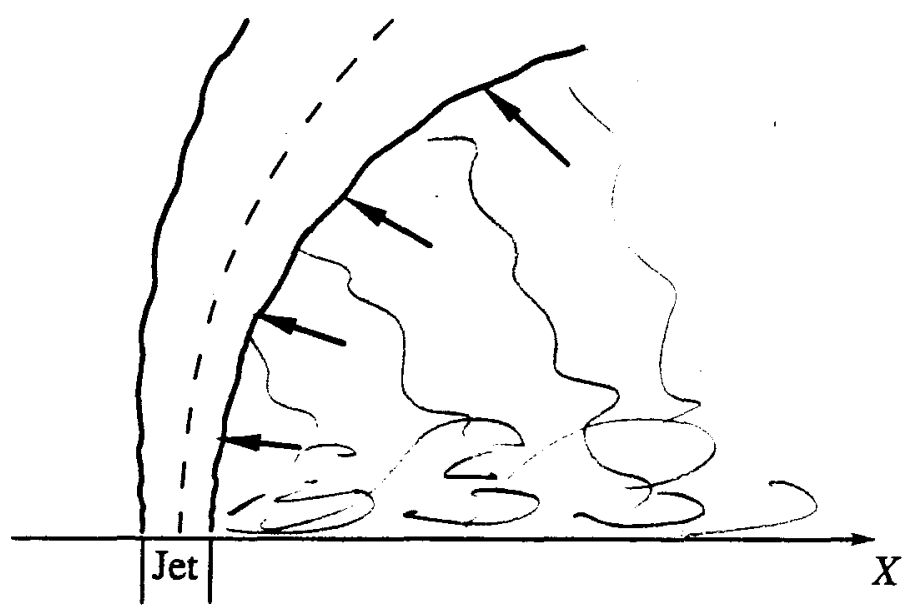

(c) $V R=8$.

FIGURE 7.6: Entrainment trajectories of the separated crossflow boundary layer fluid. (a) $V R=2$, (b) $V R=4$, (c) $V R=8$. 

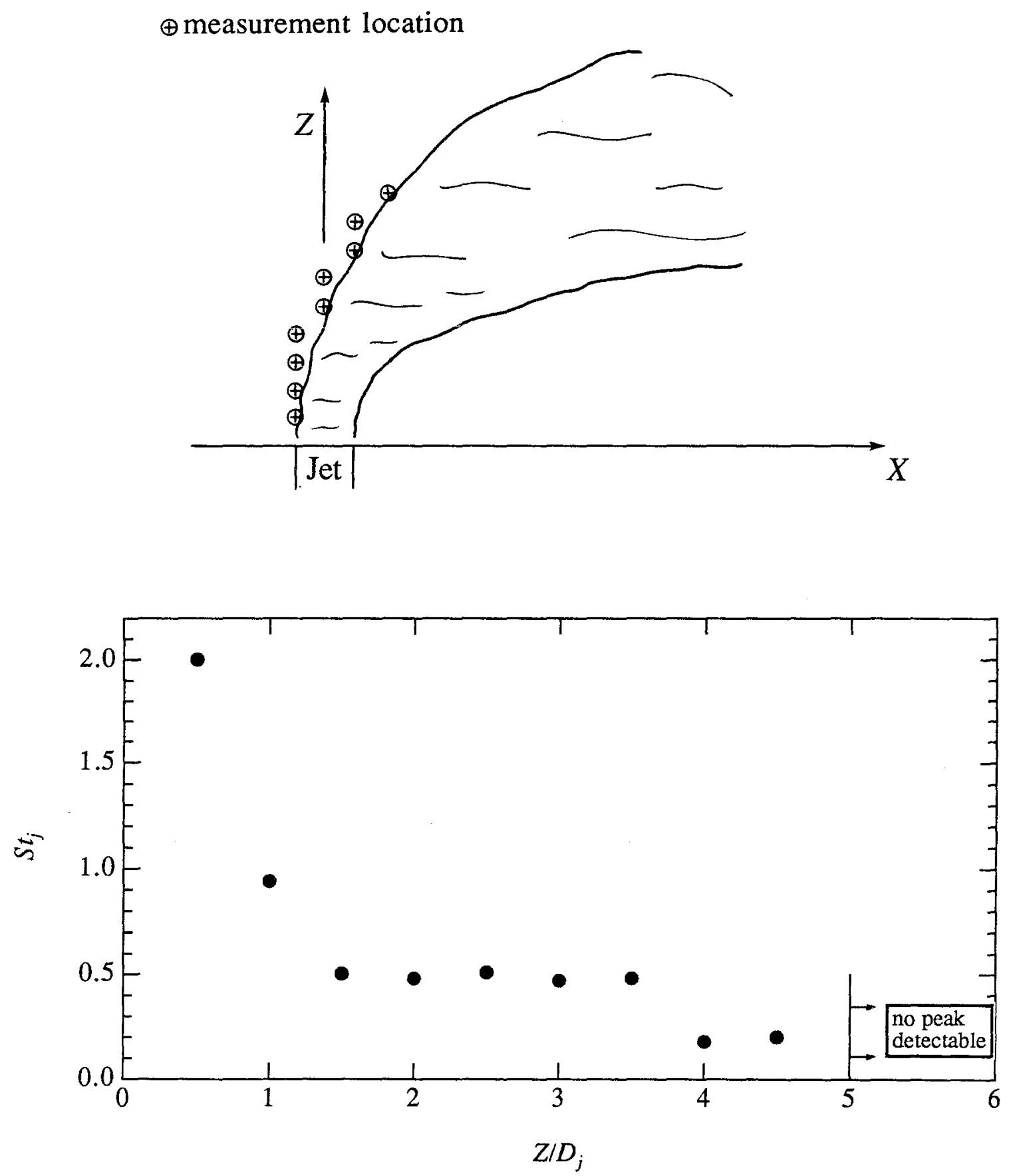

FIGURE 7.7: $S t_{j}$ along the leading edge of a transverse jet. $V R=4 . R e_{c f}=3800$. 

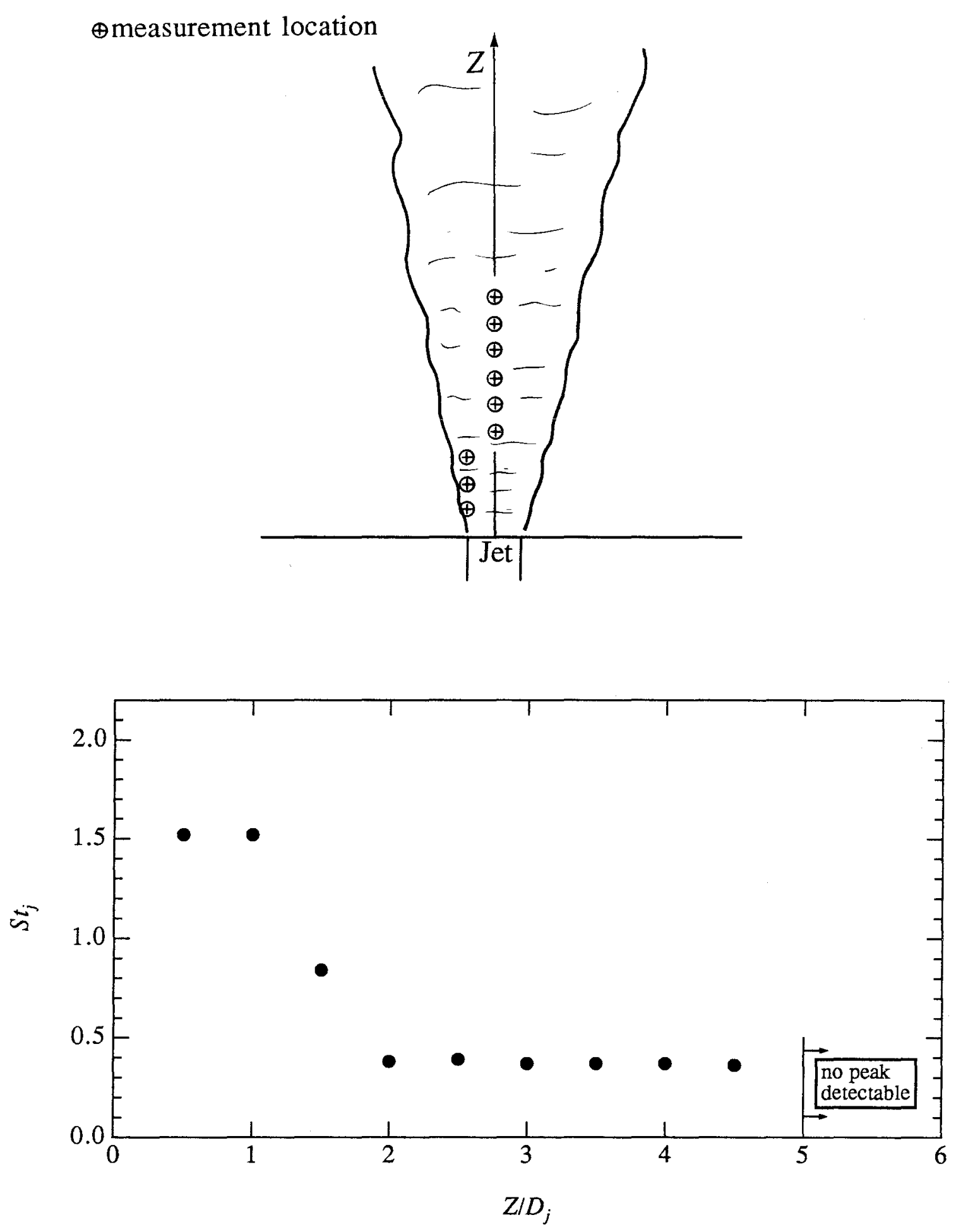

FIGURE 7.8: $S t_{j}$ along the potential core of a free jet. $R e_{j}=15200$. 

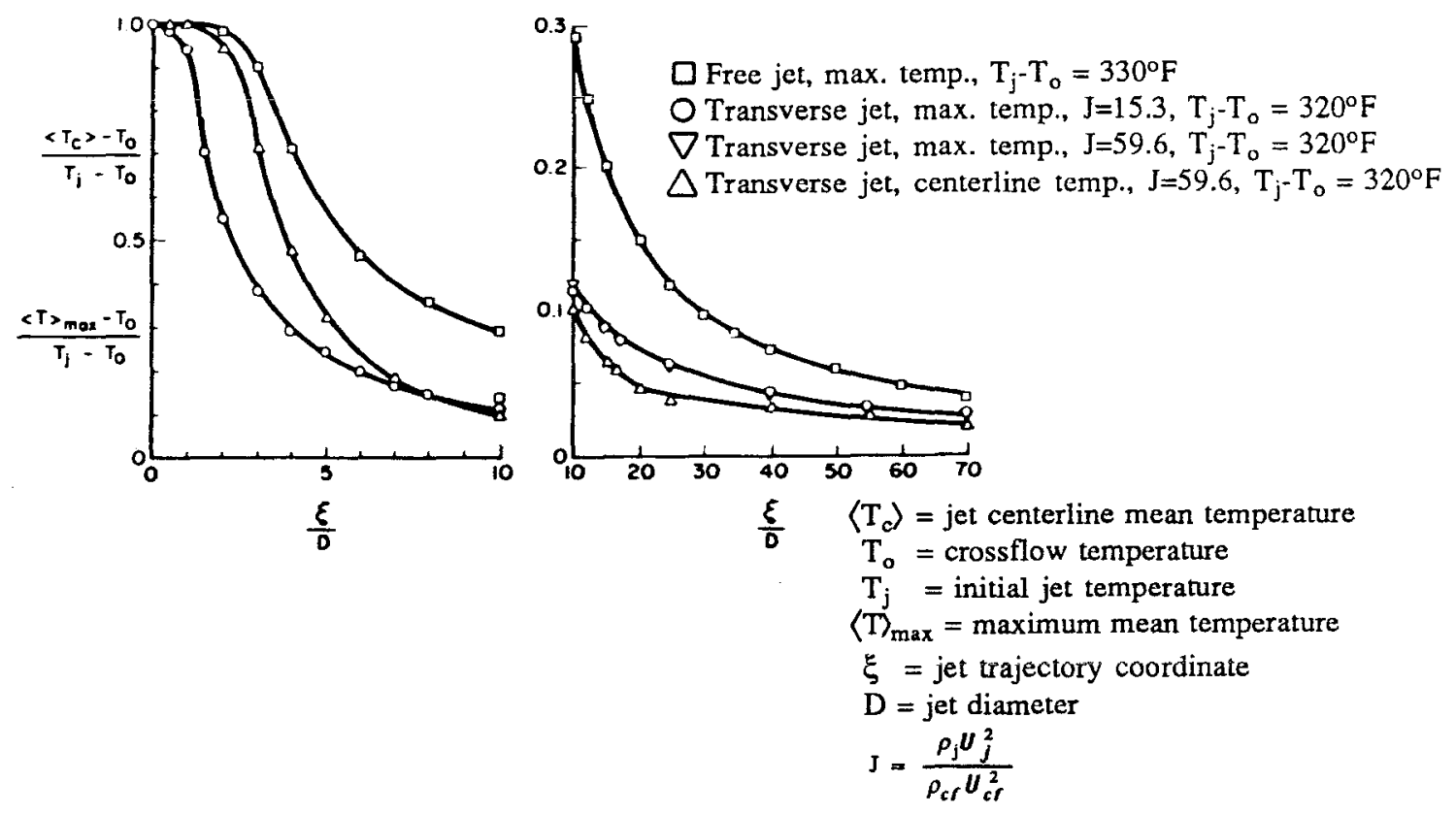

FIGURE 7.9: Decay of temperature in heated transverse and free jets. From Kamotani \& Greber (1972).

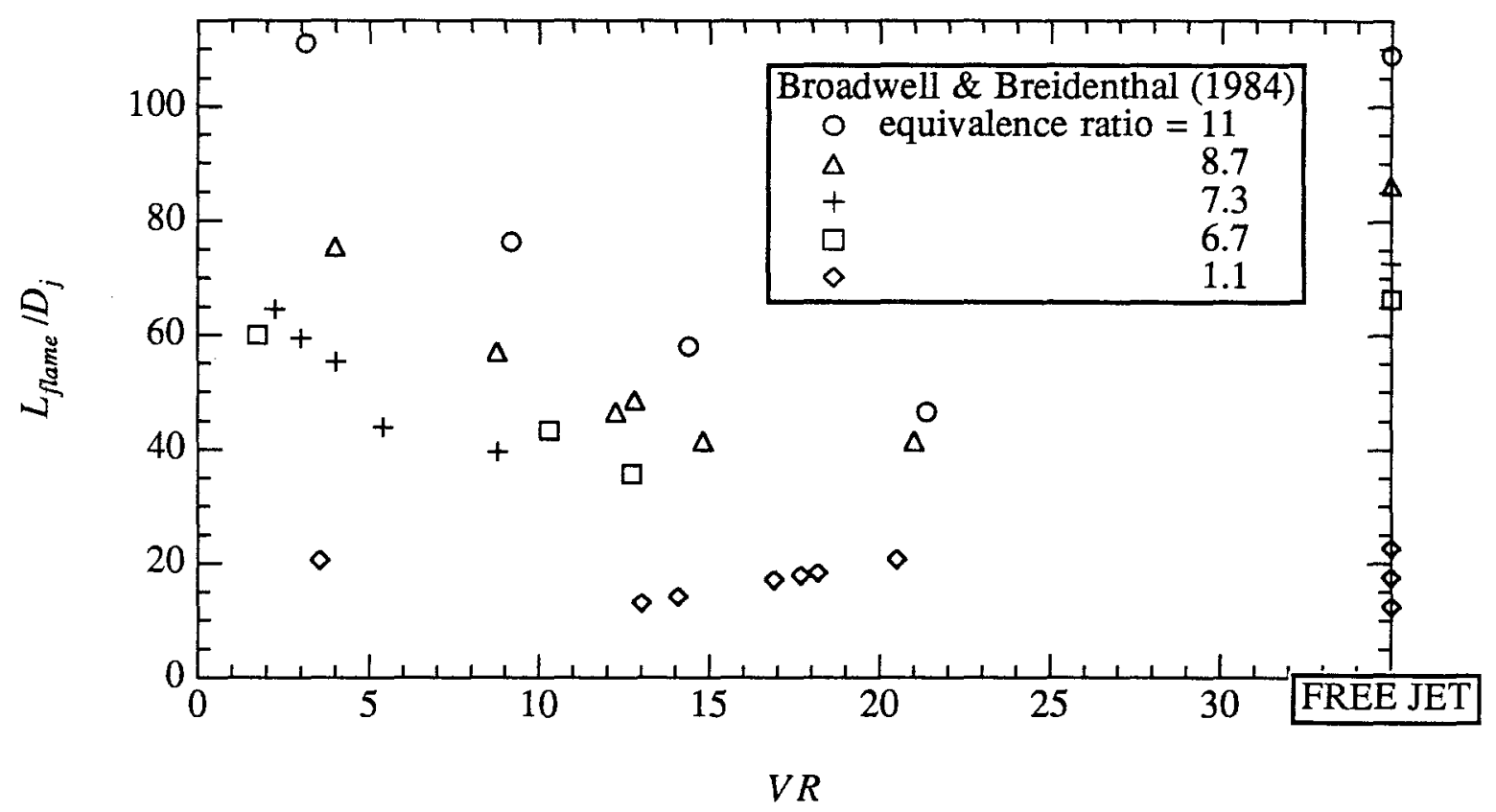

FIGURE 7.10: Reacting transverse jet and free jet flame lengths. From Broadwell \& Breidenthal (1984). 


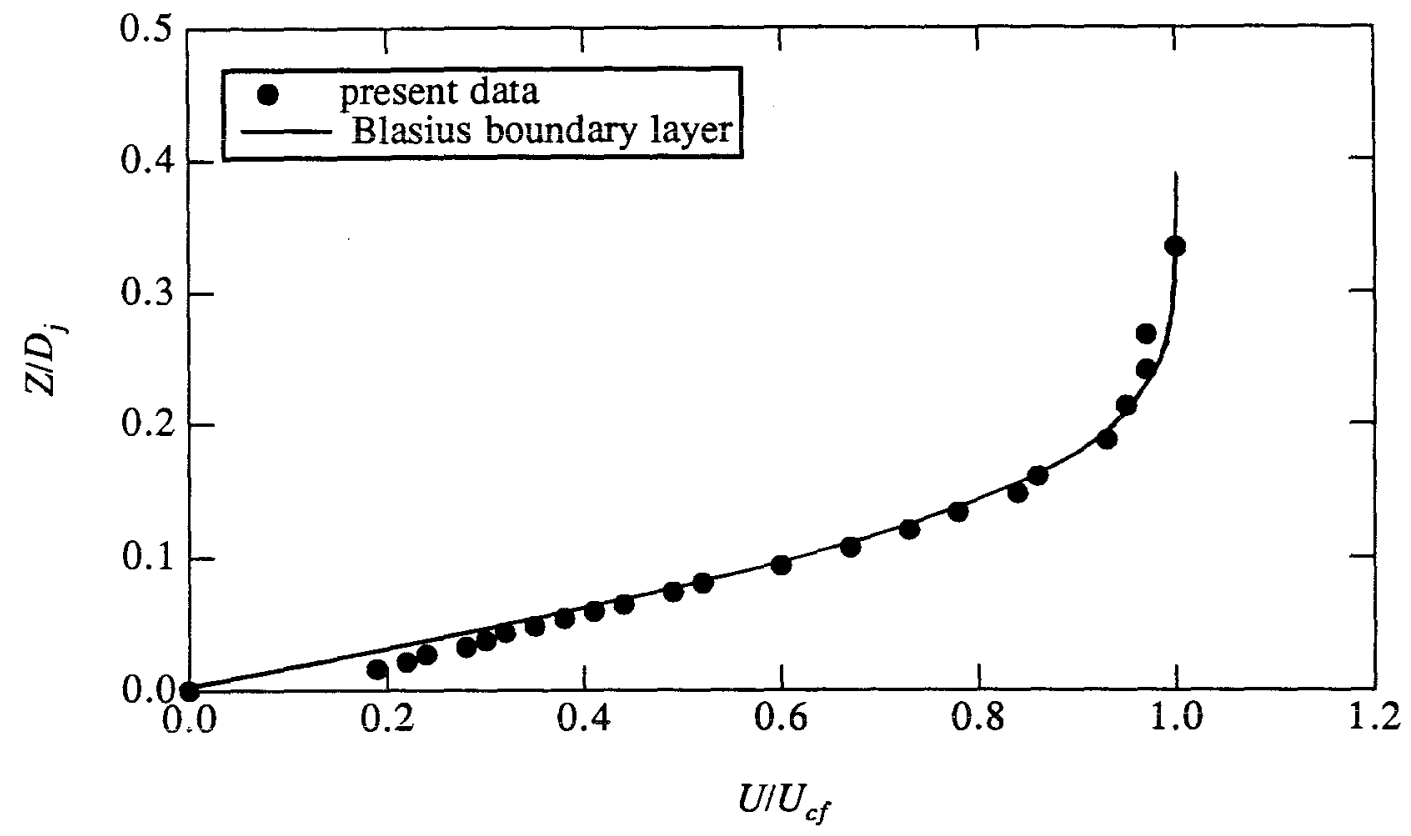

FIGURE B.1: Sample nominal crossflow boundary layer profile at $X / D_{j}=0$ showing experimental data and a Blasius profile.

$R e_{c f}=3800 . L_{j} / D_{j}=10$.

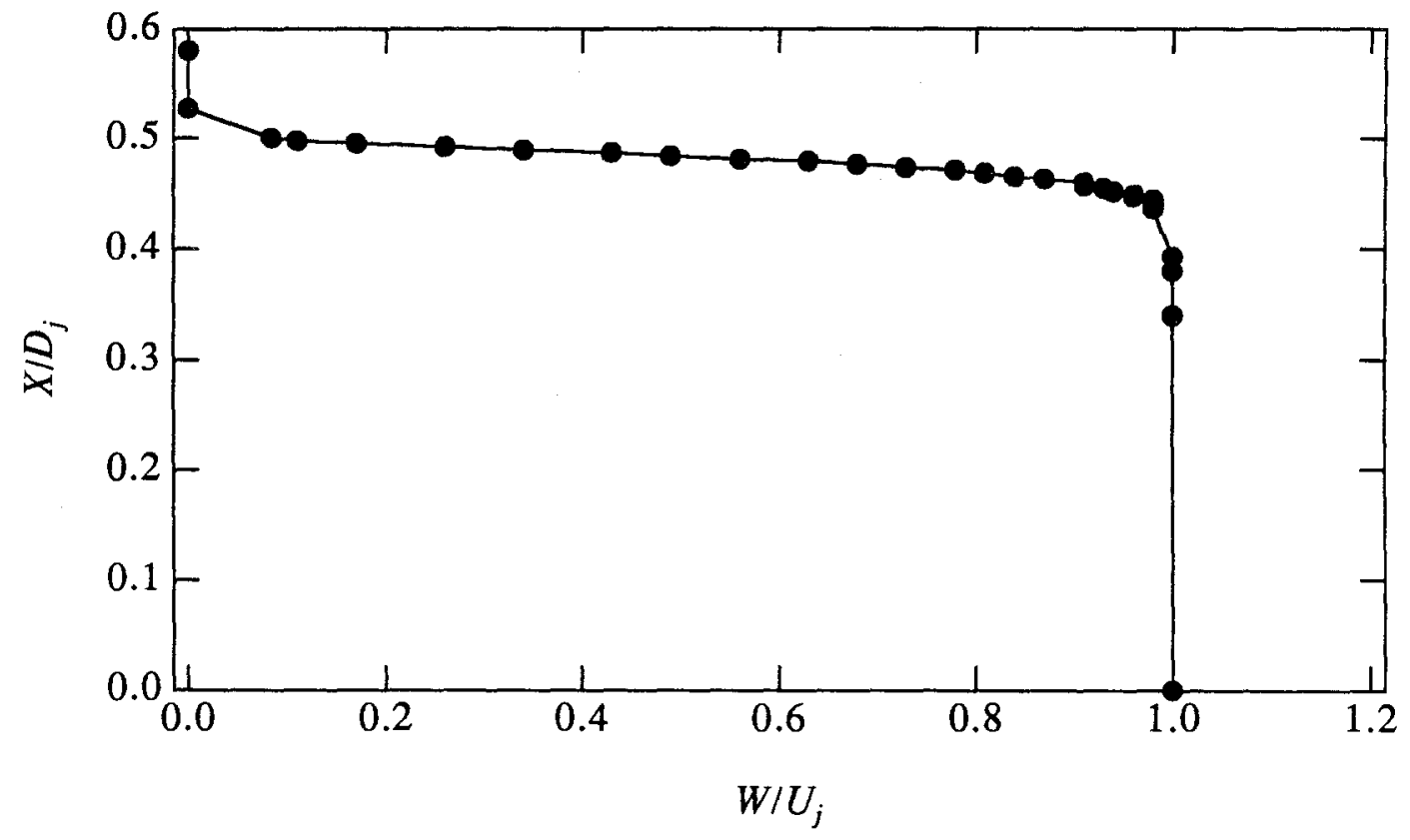

FIGURE B.2: Sample nominal jet boundary layer profile at $Z / D_{j}=.013$. Experimental data are shown. $R e_{j}=7600$. 